\title{
MICROFLUIDIC DEVICES FUNCTIONALIZED WITH PNIPAM
}

Switchable wettability for reversible emulsion generation and capillary flow control 


\section{MICROFLUIDIC DEVICES}

\section{FUNCTIONALIZED WITH}

\section{PNIPAM}

SWITCHABLE WETTABILITY FOR REVERSIBLE EMULSION GENERATION

AND CAPILLARY FLOW CONTROL 



\section{MICROFLUIDIC DEVICES FUNCTIONALIZED WITH}

\section{PNIPAM}

SWITCHABLE WETTABILITY FOR REVERSIBLE EMULSION GENERATION AND CAPILLARY FLOW CONTROL

\section{DISSERTATION}

to obtain

the degree of doctor at the University of Twente, on the authority of the rector magnificus, prof. dr. ir. A. Veldkamp, on account of the decision of the Doctorate Board to be publicly defended on Thursday 20 May 2021 at 12:45

by

\section{Lanhui Li}

born on the 30th of November, 1991 in Henan, China 
This dissertation has been approved by:

Supervisors

Prof. dr. J.C.T. Eijkel

Prof. dr. L. Shui

Co-supervisor

Prof. dr. ir. M. Odijk

Cover design: Lanhui Li

Printed by: Ridderprint / https://www.ridderprint.nl

Lay-out: Lanhui Li

ISBN: 978-90-365-5177-9

DOI: $10.3990 / 1.9789036551779$

URL: https://doi.org/10.3990/1.9789036551779

(C) 2021 Lanhui Li, The Netherlands. All rights reserved. No parts of this thesis may be reproduced, stored in a retrieval system or transmitted in any form or by any means without permission of the author. Alle rechten voorbehouden. Niets uit deze uitgave mag worden vermenigvuldigd, in enige vorm of op enige wijze, zonder voorafgaande schriftelijke toestemming van de auteur. 


\section{Graduation Committee:}

Chair / secretary:

Supervisors:

Co-supervisor:

Committee Members:
Prof. dr. J.N. Kok

Prof. dr. J.C.T. Eijkel

Prof. dr. L. Shui

Prof. dr. ir. M. Odijk

Prof. dr. A. Hibara

Prof. dr. Y. Xie

Prof. dr. F.R. Wurm

Prof. dr. J.G.E. Gardeniers

Dr. S.J.A. de Beer

Dr. E. Delamarche

The research described in this thesis was conducted at the BIOS Lab-ona-Chip Group of the MESA+ Institute for Nanotechnology, Max Planck Center for Complex Fluid Dynamics, University of Twente, Enschede, The Netherlands, and the South China Academy of Advanced Optoelectronics, South China Normal University, Guangzhou, China. The research was supported by China Scholarship Council (CSC) (Grant no. 201806750019).

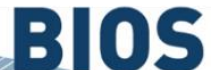

Lab on a Chip group

\section{UNIVERSITY | MESA+ OF TWENTE. INSTITUTE}
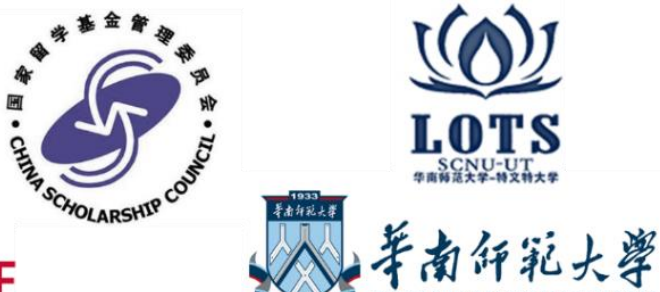



\section{Table of Contents}

\section{Chapter 1}

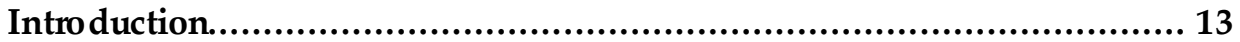

1.1 General introduction..........................................................................................

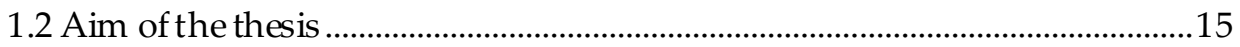

1.3 Thesis outline

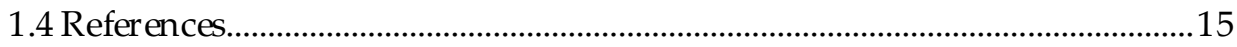

\section{Chapter 2}

In-Channel Responsive Surface Wettability for Reversible and Multiform Emulsion Droplet Preparation and Applications .................................... 19

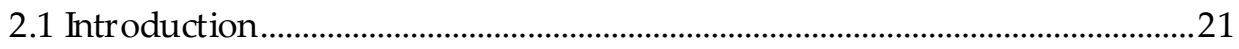

2.2 Material and methods............................................................................................23

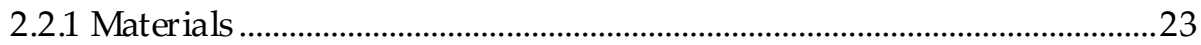

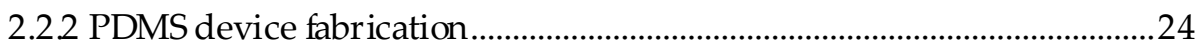

2.2.3 UV-induced surface grafting of NIPAm on PDMS mediated by

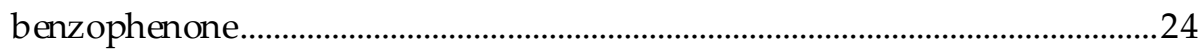

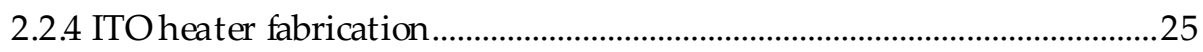

2.3 Results and discussion......................................................................................25

2.3.1 Characterization of PNIPAm-g-PDMS surface...........................................25

2.3.2 Thermally responsive surface w ettability of PNIPAm-g-PDMS..............27

2.3.3 In-channel surface treatment in microfluidic devices for reversible droplet and two-phase flow ty pes....................................................................2.

2.3.4 Surface wettability determined stable and unstable droplet formation with the same fluidic compositions in the same microfluidic device...

2.3.5 Controllable double emulsion droplet preparation in microfluidic devices by local temperature control. 
2.3.6 Synthesis of core-shell microcapsules via double emulsion droplets as

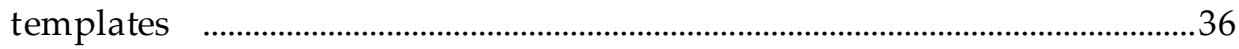

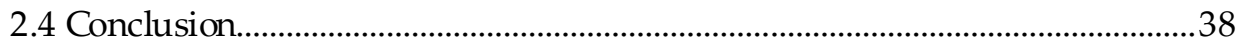

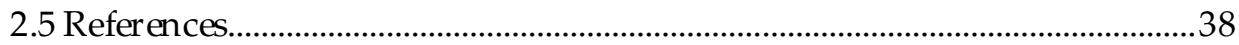

\section{Chapter3}

Autonomous Capillary Microfluidic Devices with Constant Flow Rate and Temperature-Controlled Valving........................................................45

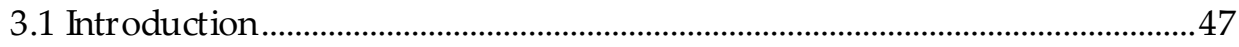

3.2 Working principle ..........................................................................................49

3.2.1 Laplace pressure and valving ……………………..................................50

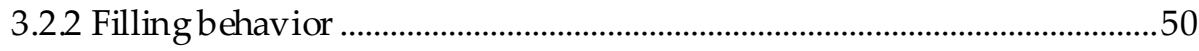

3.2.3 Temperature distribution along the channel walls at the liquid/air

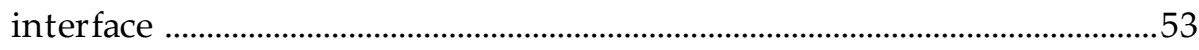

3.3 Materials and methods .......................................................................................54

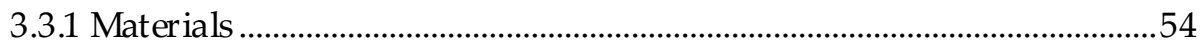

3.3.2 Heater and sensor fabrication ....................................................................55

3.3.3 Temperature sensor calibration ....................................................................5

3.3.4 PDMS device fabrication ...............................................................................5

3.3.5 UV-induced surface grafting of NIPAm on PDMS mediated by

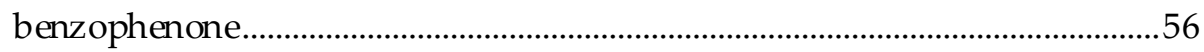

3.3.6 Contact angle measurements .......................................................................56

3.3.7 Capillary filling measurement procedure and data processing ...............57

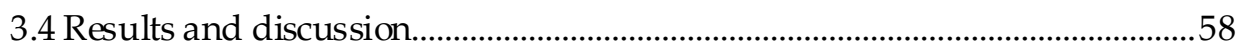

3.4.1 Surface characterization of PNIPAm-g-PDMS............................................58

3.4.2 Wetting properties of different channel walls and corresponding Laplace pressure …………...................................................................................60

3.4.3 Capillary filling behavior in the channel.......................................................63

3.4.4 Heat-triggered valve action...............................................................................64 
3.5 Conclusion and outlook

3.6 References. 66

\section{Chapter4}

On-Chip Detection of the Alcohol Concentration in Beer. 71

4.1 Introduction. .73

4.2 Working principle.

4.2.1 Phase behavior of Poly(Nisopropylacrylamide) in water/ethanol mixtures .74

4.2.2 Capillary filling behavior of water/ethanol mixtures in a PNIPAm-gPDMS microchannel .76

4.3 Materials and methods .78

4.3.1 Materials .78

4.3.2 PDMS device fabrication. .78

4.3.3 UV-induced surface grafting of NIPAm on PDMS mediated by benzophenone. 78

4.3.4 Contact angle measurements .78

4.3.5 Capillary filling measurement procedure and data processing ..............78

4.4 Results and discussion. .79

4.4.1 Wetting behavior of water/ethanol mixtures on PNIPAm-g-PDMS......79 4.4.2 Capillary filling behavior of water/ethanol mixtures in PNIPAm-gPDMS microchannels

4.4.3 Capillary filling behavior of Grolsch beer in PNIPAm-g-PDMS microchannel.

4.5 Conclusion and outlook 83

4.6 References. .84

\section{Chapter 5}

Integration of Air-pockets and Pillared Stop Valves in Microchannel for Capillary Flow Control. 


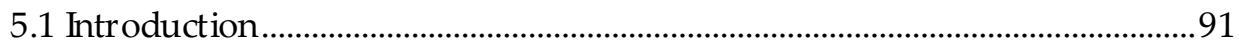

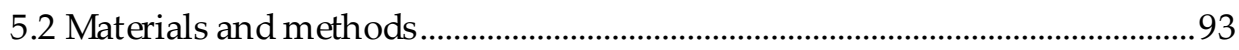

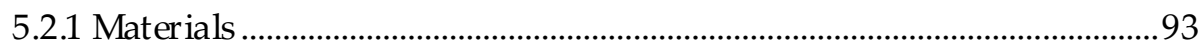

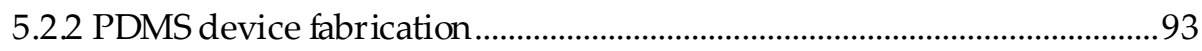

5.2.3 Chip coating with PNIPAm........................................................................94

5.2.4 Contact angle measurements .........................................................................94

5.2.5 Heater and sensor fabrication .......................................................................94

5.2.6 Temperature sensor calibration....................................................................94

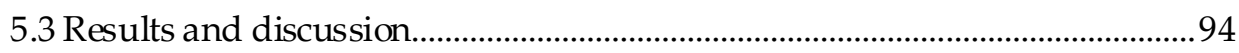

5.3.1 Contact angle (CA) of water on PNIPAm-g-PDMS pillared surface......94

5.3.2 Dimensional and temperature effects on the wetting behavior of water on PNIPAm-g-PDMS pillared surface .................................................................96

5.3.3 Temperature-controlled stop valve with pillared PNIPAm-g-PDMS for capillarity-driven flow control ............................................................................97

5.3.4 Air gate induced by thermal expansion of gas in a closed air pocket as a

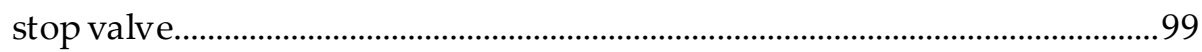

5.3.5 Integration of stop valves and air gates for capillary flow control in a

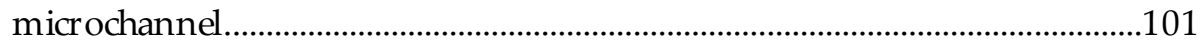

5.4 Conclusion and outlook....................................................................................103

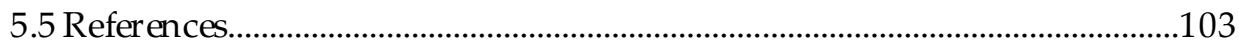

\section{Chapter 6}

High Efficiency Hydrodynamic DNA Fragmentation in a Bubbling System............................................................................ 107

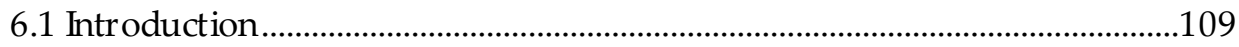

6.2 Materials and methods .........................................................................................111

6.2.1 The bubbling sy stem .................................................................................111

6.2.2 DNA sample preparation...........................................................................111

6.2.3 DNA quantification and analysis............................................................112 
6.2.4 Ligation Reaction

6.2.5 Random-amplified polymorphic DNA polymerase chain reaction (RAPD-PCR)...

6.3 Results and discussion.

6.3.1 The effect of gas Pressure...........................................................................113

6.3.2 The effect of Bubbling time.........................................................................114

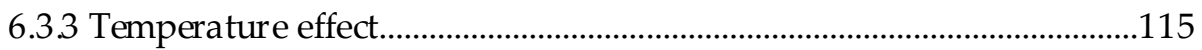

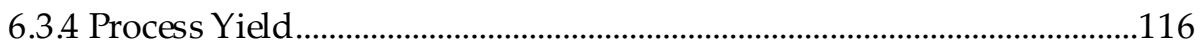

6.3.5 Ligation of Fragmented DNA..................................................................117

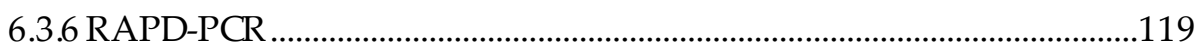

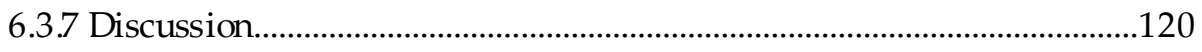

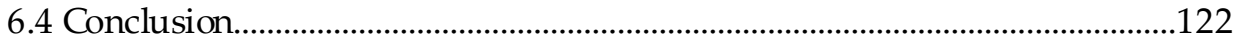

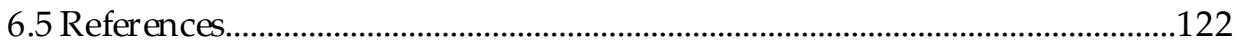

Summary and Outlook..............................................................125

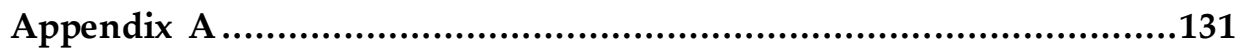

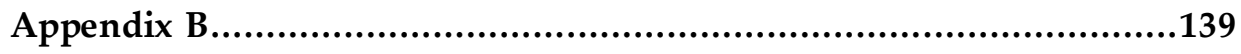

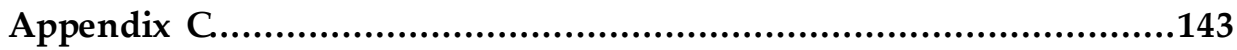

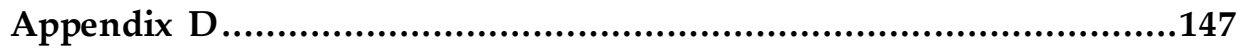

Appendix E...................................................................................153

Scientific Output.......................................................................161

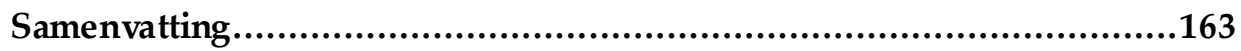

Funding and Contributions …....................................................167

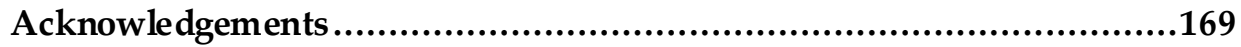





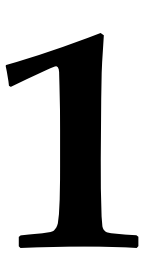

\section{Introduction}




\subsection{GENERAL INTRODUCTION}

Microfluidics provides the capability for manipulating small amounts of liquid (typically nano/microliters or less). ${ }^{1}$ Much current research is focusing on the development of cost-effective, automated microfluidic technologies for on-chip liquid manipulation. Microfluidic devices that generate monodisperse emulsion droplets of controllable volume have been widely used for microreactors, ${ }^{2}$ synthesis of nanoparticles, ${ }^{3,4}$ single-cell analysis, ${ }^{5,6}$ and drug delivery. ${ }^{7}$ Also, in recent decades more and more microfluidic technologies based on autonomous pumping, such as capillary-driven-flow-based microfluidic devices, were developed for liquid handling for Point-of-Care diagnostics. ${ }^{8}$

On downscaling to the microscale, the interfacial properties of the liquid and the solid channel wall surfaces become important for controlling liquid behavior and determine the emulsion type. ${ }^{9}$ Thus, adjusting the surface wettability of the channel surface enable opportunities for liquid handling in microfluidic devices. Various surface modification methods such as plasma oxidation, ${ }^{10} \mathrm{UV}$ treatment, ${ }^{11}$ sol-gel treatments, ${ }^{12,13}$ layer-by-layer $(\mathrm{LbL})$ assembly, ${ }^{14}$ and stimulusresponsive polymer grafting ${ }^{15-17}$ have been developed for various applications. Also, some solvent-responsive surfaces have been reported with dynamic wettability dependent on solvent components. ${ }^{18,19}$ Among all the surface modification technologies, stimulus-responsive polymers can change the surface wettability to a specific value due to the reversible chain conformation changes in response to environmental changes e.g. temperature ${ }^{20}$, light ${ }^{21}$, or solvent component such as $\mathrm{pH}^{22}$ ion concentration. ${ }^{23}$ Poly(N-isopropylacrylamide) (PNIPAm), a temperature-sensitive polymer with a lower critical solution temperature of $32{ }^{\circ} \mathrm{C}$, has been widely used for microfluidic applications for tissue engineering and liquid manipulation, due to its biocompatibility and facile operation technology with temperature. ${ }^{24,25}$ In addition to its thermal responsivity, PNIPAm is also sensitive to solutes in aqueous solution e.g. alcohol ${ }^{26,27}$ and anions especially the Hoffmeister series ${ }^{2829}$. The phase change of PNIPAm in response to the stimulus (e.g. temperature) induces a volume phase change of the PNIPAm thin film or hydrogel, resulting in a switchable wettability, which has been widely used for cell culturing, sensing, and switchable adhesive applications. ${ }^{30}$ Moreover, PNIPAm can be easily locally coated in microfluidic devices, especially PDMS-based devices, by UV-induced surface grafting ${ }^{31}$ offering flexible solutions for developing functionalized microfluidic devices. 


\subsection{AIM OF THE WORK PRESENTED IN THIS THESIS}

The work presented in this thesis results from a collaboration between the University of Twente and South China Normal University. The cooperation originally started with developing microfluidic devices for DNA sample manipulation and then was changed to developing microfluidic devices with fast switching surface wettability between hydrophilic and hydrophobic, aiming at controlling emulsion type and manipulating capillary flow.

\subsection{THESIS OUTLINE}

This thesis in chapters 2-5 presents several microfluidic technologies for applications mainly in two fields: reversible emulsion generation and capillary flow control. In chapters 2, 3, 4, and 5, PDMS w as used as the material for device fabrication. PNIPAm was grafted on the channel surface to obtain reversible hydrophilic/hydrophobic surface wettability. Throughout this thesis, we call these devices PNIPAm-g-PDMS device. Chapter 2 studies the reversible wettability of PNIPAm-g-PDMS microfluidic devices and presents a method for preparing reversible emulsion and multiform emulsion generation. Chapter 3 presents a PNIPAm-g-PDMS capillary microfluidic device with a constant filling flow rate and temperature-controlled valving in a temperature range of 20 to $40{ }^{\circ} \mathrm{C}$. In Chapter 4, we further study the effects of ethanol on the wetting behavior of $w$ ater on PNIPAm-g-PDMS surfaces and present a device for alcohol concentration detection in beer; Chapter 5 is a further development of the technology in chapter 3 , where we developed a technology for on-chip capillary flow control by integrating pillared PNIPAm-g-PDMS stop valve and air gates. The PhDresearch originally started with work on microfluidics for DNA sample preparation, and the PhD aim was later changed to the study of PNIPAm in microfluidic devices. Chapter 6 presents w ork from the initial period, where we use bursting bubbles for DNA fragmentation. At the end of the thesis in Chapter 7 , a summary of all the chapters and recommendations for future work are presented.

\subsection{REFERENCES}

1. Tarn, M. D. \& Pamme, N. Microfluidics. in Reference Module in Chemistry, Molecular Sciences and Chemical Engineering (eds. Ligler, F. S. \& Taitt, C. R. B. T.-O. B. (Second E.) 659-681 (Elsevier, 2014).

2. Das, S. \& Srivastava, V.C. Microfluidic-based photocatalytic microreactor for environmental application: a review of fabrication substrates and 
techniques, and operating parameters. Photochem. Photobiol. Sci. 15, 714-730(2016).

3. Abalde-Cela, S., Taladriz-Blanco, P., de Oliveira, M. G. \& Abell, C. Droplet microfluidics for the highly controlled synthesis of branched gold nanoparticles. Sci. Rep. 8, 2440 (2018).

4. Frenz, L. et al. Droplet-Based Microreactors for the Synthesis of Magnetic Iron Oxide Nanoparticles. Angew. Chemie Int. Ed. 47, 6817-6820 (2008).

5. Luo, T., Fan, L., Zhu, R. \& Sun, D. Microfluidic Single-Cell Manipulation and Analysis: Methods and Applications. Micromachines 10,104(2019).

6. Kang, D. K., Monsur Ali, M., Zhang, K., Pone, E. J. \& Zhao, W. Droplet microfluidics for single-molecule and single-cell analysis in cancer research, diagnosis and therapy. Tr AC - Trends in Analytical Chemistry (2014).

7. Zhao, C.-X. Multiphase flow microfluidics for the production of single or multiple emulsions for drug delivery. Adv. Drug Deliv. Rev. 65, 14201446 (2013).

8. Jung, W., Han, J., Choi, J.-W. \& Ahn, C. H. Point-of-care testing (POCT) diagnostic systems using microfluidic lab-on-a-chip technologies. Microelectron. Eng. 132, 46-57 (2015).

9. Shui, L., van den Berg, A. \& Eijkel, J. C. T. Interfacial tension controlled W/O and O/W 2-phase flows in microchannel. Lab Chip 9, 795-801 (2009).

10. Fritz, J. L. \& Owen, M. J. Hydrophobic Recovery of Plasma-Treated Polydimethylsiloxane. J. Adhes. 54,33-45 (1995).

11. Trantidou, T., Elani, Y., Parsons, E. \& Ces, O. Hydrophilic surface modification of PDMS for droplet microfluidics using a simple, quick, and robust method via PVAdeposition. Microsystems Nanoeng. 3, 16091 (2017).

12. Roman, G. T., Hlaus, T., Bass, K. J., Seelhammer, T. G. \& Culbertson, C. T. Sol-Gel Modified Poly(dimethylsiloxane) Microfluidic Devices with High Electroosmotic Mobilities and Hydrophilic Channel Wall Characteristics. Anal. Chem. 77, 1414-1422 (2005).

13. Abate, A. R., Lee, D., Do, T., Holtze, C. \& Weitz, D. A. Glass coating for PDMS microfluidic channels by sol-gel methods. Lab Chip 8, 516 (2008).

14. Choi, C.-H., Lee, H. \& Weitz, D. A. Rapid Patterning of PDMS Microfluidic Device Wettability Using Syringe-Vacuum-Induced Segmented Flow in Nonplanar Geometry. ACS Appl. Mater. Interfaces 10, 3170-3174(2018).

15. Hu, S. et al.Surface Modification of Poly (dimethylsiloxane) Microfluidic Devices by Ultraviolet Polymer Grafting. Anal. Chem. 74, 4117-4123 (2002). 
16. Schneider, M. H., Willaime, H., Tran, Y., Rezgui, F. \& Tabeling, P. Wettability Patterning by UV-Initiated Graft Polymerization of Poly (acrylic acid) in Closed Microfluidic Sy stems of Complex Geometry. Anal. Chem. 82, 8848-8855 (2010).

17. $\mathrm{Mu}, \mathrm{M}$. \& Ebara, M. Smart polymers. in Polymer Science and Nanotechnology (ed. Narain, R. B. T.-P.S. and N.) 257-279 (Elsevier, 2020).

18. Fukuyama, M., Tokeshi, M., Proskurnin, M. A. \& Hibara, A. Dynamic wettability of polyethylene glycol-modified poly(dimethylsiloxane) surfaces in an aqueous/organic two-phase system. Lab Chip 18, 356-361 (2018).

19. Mu, M. \& Ebara, M. Smart polymers. in Polymer Science and Nanotechnology (ed. Narain, R. B. T.-P.S. and N.) 257-279 (Elsevier, 2020). doi:10.1016/B978-0-12-816806-6.00012-1

20. Kim YJ, Matsunaga YT. Thermo-responsive polymers and their application as smart biomaterials. Journal of Materials Chemistry B. 5(23):4307-21(2017).

21. Motornov, M., Sheparovych, R., Tokarev, I., Roiter, Y. \& Minko, S. Nonwettable Thin Films from Hybrid Polymer Brushes Can Be Hydrophilic. Langmuir 23, 13-19 (2007).

22. Kocak G, Tuncer CA, Bütün VJ. pH-Responsive polymers. Polymer Chemistry. 8(1):144-76 (2017).

23. Wang, Y. et al. Covalent Micropatterning of Poly (dimethylsiloxane) by Photografting through a Mask. Anal. Chem. 77, 7539-7546 (2005).

24. Da Silva, R. M. P., Mano, J. F. \& Reis, R. L. Smart thermoresponsive coatings and surfaces for tissue engineering: switching cell-material boundaries. Trends Biotechnol. 25, 577-583(2007).

25. Patel, N. G. \& Zhang, G. Responsive systems for cell sheet detachment. Organogenesis 9, 93-100 (2013).

26. Winnik, F. M., Ringsdorf, H. \& Venzmer, J. Methanol-water as a cononsolvent system for poly(N-isopropylacrylamide). Macromolecules 23, 2415-2416(1990).

27. Schild,H. G., Muthukumar, M. \& Tirrell, D. A. Cononsolvency in mixed aqueous solutions of poly (N-isopropylacrylamide). Macromolecules 24, 948-952(1991).

28. Zhang, Y., Furyk, S., Bergbreiter, D. E. \& Cremer, P. S. Specific ion effects on the w ater solubility of macromolecules: PNIPAM and the Hofmeister series. J. Am. Chem. Soc. (2005).

29. Zajforoushan Moghaddam, S. \& Thormann, E. Hofmeister Effect on PNIPAM in Bulk and at an Interface: Surface Partitioning of Weakly Hydrated Anions. Langmuir 33, 4806-4815 (2017). 
30. Yu, Y., Brió Pérez, M., Cao, C. \& de Beer, S. Switching (bio-) adhesion and friction in liquid by stimulus responsive polymer coatings. Eur. Polym. J. 147, 110298 (2021).

31. Hu, S. et al. Surface-Directed, Graft Polymerization within Microfluidic Channels. Anal. Chem. 76, 1865-1870 (2004). 


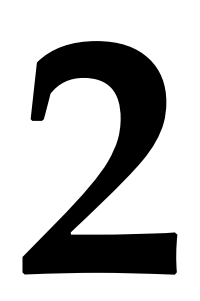

In-Channel Responsive Surface Wettability for Reversible and Multiform Emulsion

Droplet Preparation and Applications 
$\mathcal{S}_{\text {surface-to-volume ratio in microfluidic devices. As a consequence, achieving }}^{\text {urface wettability plays a crucial role in microfluidic systems due tothe large }}$ active control of surface w ettability has become a paradigm for the formation of emulsions in microfluidic devices. In this chapter, we report a simple approach for in-channel functionalization of a polydimethylsiloxane (PDMS) surface to obtain a switchable and reversible wettability change between hydrophilic and hydrophobic states. The thermally responsive polymer, Poly $(\mathrm{N}$ Isopropylacrylamide) (PNIPAm), $w$ as grafted on the surface of PDMS channels by UV-induced surface grafting. The surface wettability of PNIPAm grafted PDMS (PNIPAm-g-PDMS) can be thermally tuned to obtain water contact angles varying in the range of 24.3 to $106.1^{\circ}$ by varying the temperature between $25-$ $38{ }^{\circ} \mathrm{C}$. By selectively modifying the functionalized area in the microfluidic channels, multiform emulsion droplets of oil-in-water $(\mathrm{O} / \mathrm{W})$, water-in-oil $(\mathrm{W} / \mathrm{O})$, oil-in-water-in-oil $(\mathrm{O} / \mathrm{W} / \mathrm{O})$, and water-in-oil-in-water $(\mathrm{W} / \mathrm{O} / \mathrm{W})$ could be created on-demand. Combining solid surface wettability and liquid-liquid interfacial properties, tunable generation of $\mathrm{O} / \mathrm{W}$ and $\mathrm{W} / \mathrm{O}$ droplets and stratified flows were enabled in the same microfluidic device with either different or the same two-phase fluidic systems, by properly heating/cooling thermally responsive microfluidic channels and by choosing suitable surfactants. Controllable creation of $\mathrm{O} / \mathrm{W} / \mathrm{O}$ and $\mathrm{W} / \mathrm{O} / \mathrm{W}$ droplets $\mathrm{w}$ as also achieved in the same microfluidic device, by locally heating or cooling the droplet generation areas with integrated electric heaters to achieve opposite surface w ettability. Hollow microcapsules were prepared using double emulsion droplets as templates in the microfluidic device with sequential hydrophobic and hydrophilic channel segments, demonstrating the strength of the proposed approach in practical applications.

tThis chapter is based on the publication: L. Li, Z. Yan, M. Jin, X. You, S. Xie, Z. Liu, A. van den Berg, J. C.T. Eijkel, L. Shui. In-Channel Responsive Surface Wettability for Reversible and Multiform Emulsion Droplet Preparation and Applications. ACS applied materials \& interfaces. 2019, 11(18), 16934-16943. 


\subsection{INTRODUCTION}

Wettability is one of the key factors related to functionalities in biological system 1 , cell membrane remodeling, ${ }^{2}$ water transport in earth soil, ${ }^{3}$ w etting behavior of liquid on surfaces, ${ }^{4}$ protein, and bimolecular adsorption, ${ }^{5}$ nanomaterial synthesis, ${ }^{6}$ and sustainability of photoelectric devices. ${ }^{7}$ Surface wettability is also a key factor in handling complex fluidic systems involving both polar and nonpolar fluids, such as emulsions. Emulsions composed of two or more immiscible liquids are used in a wide range of industries such as cosmetic, food, agriculture, and pharmaceutical industries. ${ }^{8}$ Emulsion types basically including $\mathrm{O} / \mathrm{W}, \mathrm{W} / \mathrm{O}$, $\mathrm{O} / \mathrm{W} / \mathrm{O}$, and $\mathrm{W} / \mathrm{O} / \mathrm{W}$ emulsions have been well developed for preparing stable micro/nano-droplets or microcapsules, which are applied as food emulsion, or for topical applications such as for diagnostics and drug delivery. ${ }^{9,10}$ However, for some special applications in food industry and petrochemical industry, emulsion reversion is a necessity that still in need of more study. $8,11,12$

Droplet-based microfluidics has emerged as a popular technology to generate multiform emulsions for a wide range of applications with high throughput such as encapsulation, chemical synthesis, and biochemical assays.13,14 Generally, droplet-based microfluidic systems create discrete volumes that serve as confined spaces serving as microreactors, microcages, etc. The size of the generated droplets is mainly controlled by the fluid viscosity, flow rate, interfacial tension between the immiscible phases, flow geometry, and the surface wettability of the microfluidic channels used for droplet generation. ${ }^{13,15,16}$ On downscaling to the microscale level, the high surface-to-volume ratio causes an increasing role of interfacial properties either between the immiscible fluidic phases coexisting in a microchannel or between the fluidic phaseand the channel wall. ${ }^{13,17}$ Controlling the surface wettability of the microfluidic channel is therefore important when creating either $\mathrm{W} / \mathrm{O}$ or $\mathrm{O} / \mathrm{W}$ emulsion droplets or for the formation of double or multiple emulsions. W/O droplets are generally formed in a hydrophobic microchannel wetting the oil phase, whereas $\mathrm{O} / \mathrm{W}$ droplets can be obtained in a hydrophilic channel.13,18 To form a double emulsion, a two-step process ${ }^{19,20}$ or local modification of microfluidic channels ${ }^{21-23}$ is required to form two droplet generators with opposite w et tability. ${ }^{24,25}$

Microfluidic devices are commonly fabricated in silicon, glass, or polymeric materials such as polydimethylsiloxane (PDMS), cy clic olefin copolymer (COC), or poly(methyl methacrylate) (PMMA) using standard photolithography, softlithography, mechanical milling, or laser writing methods. ${ }^{26}$ Among these 
materials, PDMS-based microfluidic devices are especially popular, primarily due to their ease of fabrication, optical transparency, biological inertness, nontoxicity, and gas permeability. ${ }^{2,28}$ However, due to its intrinsic hydrophobicity, PDMS encounters significant limitations in producing stable $\mathrm{O} / \mathrm{W}$ emulsion droplets. The microchannel surface must be treated to be hydrophilic to ensure effective wetting to the continuous aqueous solution, and the result of this treatment should be stable in time. ${ }^{29}$

Various surface modification methods have been investigated toachieve surface wettability control of PDMS microfluidic devices. Plasma oxidation is by far the most commonly used method which oxidizes the PDMS surface to create a hydrophilic silica surface layer; how ever, this method only leads to surface roughness and a temporary hydrophilic surface, as PDMS regains its original surface properties over time. ${ }^{30} \mathrm{UV}$ treatment facilitates much deeper modification of the PDMS surface without inducing cracks or mechanically weakening PDMS; how ever, this effect is also transient. ${ }^{31}$ Sol-gel treatments result in a glass-like layer on the PDMS surface; however, they are difficult to handle due to the complicated modification process which also limits their applications for simple geometries. ${ }^{32,33}$ Layer-by-layer (LbL) assembly deposits positively and negatively charged polymers onto the PDMS surface to successfully render it hydrophilic; however, the coating process is timeconsuming and difficult to control due to its chemical sensitivity. ${ }^{2829,34}$ Photoinitiated grafting has been applied for surface modification with the advantages of allowing local modification of selected areas in channels with few steps. 2835 Various materials, such as acrylic acid, ${ }^{35-37}$ acrylamide, ${ }^{35}$ poly(ethylene glycol), ${ }^{3839}$, and $\mathrm{N}$-isopropylacrylamide, ${ }^{40-42}$ have furthermore been reported to be graftedon the PDMS surface via photo-induced grafting, to render it hy drophilic.

Poly (N-isopropylacrylamide) (PNIPAm) is well-known as a thermo-responsive polymer and has been applied as a thermo-responsive surface for cell culturing and harvesting. ${ }^{40,43}$ PNIPAm changes its structure in response to temperature variation in aqueous solutions. The polymer chains swelling in water at a temperature below its lower critical solution temperature (LCST) of $32{ }^{\circ} \mathrm{C}$ and forming compact structures that can aggregate and precipitate from solution at a temperature above its LCST. ${ }^{44}$ As a result, the w ettability of PNIPAm surfaces changes with the molecular structure in response to temperature variation, show ing hydrophilic and hy drophobic properties at the temperature below and above LCST, respectively. ${ }^{45,46}$ The switchable wettability of PNIPAm surfaceshas 
been employed in various functional material 4748 and tissue engineering fields. ${ }^{40,49-51}$

In this w ork, PDMS-based microfluidic channels were modified by in-channel UV-induced grafting of NIPAm to show thermally responsive wettability. The preparation of PDMS microfluidic devices and the surface modification process are demonstrated step-by-step. The properties and quality of the coatings at different stages were investigated by using Fourier transform infrared spectroscopy (FTIR) for chemical group characterization, contact angle measurement for surface wettability determination, and Scanning Electron Microscope (SEM) and Atomic Force Microscope (AFM) for surface topology characterization. The effect of UV initiating time on surface wettability modification was studied. The thermal wettability response of the prepared PNIPAm grafted PDMS (PNIPAm-g-PDMS) was subsequently investigated, showing a large change of water contact angle (CA) by slightly varying temperature. Modification of a flat PDMS surface, entire microfluidic channels, and local parts of microfluidic channels have all been demonstrated. Emulsion droplets of $\mathrm{O} / \mathrm{W}, \mathrm{W} / \mathrm{O}, \mathrm{O} / \mathrm{W} / \mathrm{O}$, and $\mathrm{W} / \mathrm{O} / \mathrm{W}$ could be produced in a single microfluidic device by adjusting the temperature of the grafted surfaces. Such a flexible control over droplet types is much beneficial for applications such as the production of solid microspheres and core-shell microcapsules of various materials.

\subsection{MATERIALS AND METHODS}

\subsubsection{Materials}

N-Isopropylacrylamide (NIPAm, CAS: 2210-25-5), benzophenone (CAS: 119-619), $\mathrm{NaIO}_{4}$ (CAS: 7790-28-5), benzyl alcohol (CAS: 100-51-6), and Ethoxylated trimethylolpropane triacrylate (ETPTA) were all obtained from Sigma-Aldrich (Shanghai, China). 2-dimethoxy-2-phenylacetophenone (Heowns Co., Tianjin, China) was used as a photoinitiator. Deionized(DI) w ater $\left(18.2 \mathrm{M} \Omega\right.$ at $\left.25^{\circ} \mathrm{C}\right)$ was prepared using a Milli-Q Plus water purification system (Milli-Q Plus water purification, Sichuan Wortel Water Treatment Equipment Co. Ltd, Sichuan, China). Negative photoresist SU-8 3050 and developer solution w ere purchased from MicroChem (MA, USA) for fabricating the silica mold with designed microchannels. Positive photoresist SUN-120P (Suntific Microelectronic Materials Co. Ltd, Weifang, China) was used for fabricating the ITO patterns. The poly (dimethylsiloxane) (PDMS, Sylgard 184) package was purchased from Dow 
Corning Corporation (Midland-Michigan, USA) and was used for fabricating the microfluidic chip. ITO-coated glass slides (Shenzhen Laibao Hi-tech Co. Ltd., China) were used to fabricate ITO heaters. Silver Conductive Epoxy was purchased from MG Chemicals (Surrey, BC, Canada).

\subsubsection{PDMS device fabrication}

Designed microchannel patterns were transferred on a SU-8 (negative photoresist) layer spin-coated on a silicon wafer (Lijing Optoelctronics Co. Ltd, Suzhou, China), to serve as master mold using a standard photolithography technique.52 PDMS pre-polymer and curing agent were mixed using a stirring machine at a mass ratio of 10:1 and then degassed in a vacuum chamber. The mixture was then cast onto the master mold and thermally cured in a heater at $90{ }^{\circ} \mathrm{C}$ for $0.5 \mathrm{~h}$. The PDMS replica with designed channel patterns $\mathrm{w}$ as then peeled from the silicon master and cut into the predesigned size. ThePDMS with designed channel patterns was bonded with a block of $1 \mathrm{~mm}$ thick flat PDMS layer after $\mathrm{O}_{2}$ plasma bonding in a plasma cleaner (PDC-002, MycroTechnologies Co., Ltd, Beijing, China) at $27 \mathrm{~W}$ power for $3 \mathrm{~min}$ to form a microfluidic chip. The schematic drawing of this process is shown in Figure A1 in Appendix A.1.

2.2.3 UV-induced surface grafting of NIPAm on PDMS mediated by benzophenone

UV-induced grafting of PNIPAm was conducted according to the method reported by Ebara et al.. ${ }^{40}$ Benzophenone, a photosensitizer, $w$ as dissolved in acetone (20 wt \%). $1 \mathrm{~mm}$ thick flat PDMS film was immersed in the benzophenone-acetone solution for $10 \mathrm{~min}$ at room temperature to let the photosensitizer be fully absorbed intoPDMS. Afterward, the piece of PDMS film w as thoroughly rinsed with ethanol and water. The other layer of PDMS film with a thickness of $3 \mathrm{~mm}$ was then prepared. Two PDMS films w erealigned and integrated with a gap distance of $60 \mu \mathrm{m}$ controlled by a PDMS spacer with known thickness. The monomer solution containing NIPAm $(10 \mathrm{wt} \%), \mathrm{NaIO}_{4}(0.5$ $\mathrm{mM})$, benzyl alcohol $(0.5 \mathrm{wt} \%) \mathrm{w}$ as then filled into the gap between the two PDMS films by capillary filling. This device was subsequently placed in an oven with a UV irradiator $(210 \mathrm{~W}, 365 \mathrm{~nm}$, Intelliray 600, Uvitron International Inc, USA) to graft the PNIPAm onto the PDMS surfaces, and then immersed and washed in DI water to remove residual monomer and polymer. An ice-water bath was then used to keep the temperature of the monomer solution below the LCST of NIPAm. 


\subsubsection{ITOheater fabrication}

A patterned Indium tin oxide (ITO) heater was fabricated by standard photolithography and a wet-etching process, as shown in Figure A2 (See Appendix A). ITO-glass was first ultrasonically washed withDI w ater and 99.5\% ethanol, cleaned with $\sim 4 \mathrm{wt} \%$ alkaline cleaning agent, and then rinsed with deionized water and blow-dried using a nitrogen gun. Afterward, the cleaned ITO-glass was spin-coated by a photoresist (SUN-120P) layer and then exposed to UV $\left(27 \mathrm{~mW} / \mathrm{cm}^{2}\right)$ for $30 \mathrm{~s}$ through a patterned transparent photomask. The heater patterns were developed in a $4 \mathrm{wt} \% \mathrm{KOH}$ solution. ITO etching was performed by immersing the photoresist patterned slides in an aqueous solution containing $\mathrm{HNO}_{3}, \mathrm{HCl}$, and $\mathrm{H}_{2} \mathrm{O}$ (with a volume ratio of $3: 50: 50$ ) at $50{ }^{\circ} \mathrm{C}$ for 1.5 min. SUN-120P was then removed by dissolving in ethanol. The electrical conductors were fabricated by coating the patterned heater with $5 \mathrm{~nm}$ of chromium followed by sputtering $35 \mathrm{~nm}$ of gold (AJA International. ATC Orion 8 , USA) covered with a shadow mask.

\subsection{RESULTS AND DISCUSSION}

\subsubsection{Characterization of PNIPAm-g-PDMS surface}

Bare PDMS consists of repeating - $\mathrm{OSi}\left(\mathrm{CH}_{3}\right)_{2}$ - units on the surface. The - $\mathrm{CH} 3$ groups result in poor surface wettability to water with a contact angle of about $106 \pm 0.4^{\circ}$. UV irradiation induces the generation of free radicals which cause NIPAm monomers to polymerize on the treated PDMS surface. ${ }^{35,37}$ Figure 2.1a and $b$ illustrate the initial PDMS surface and the PDMS with the grafted PNIPAm layer after UV irradiation. ${ }^{53}$ In Figure 2.1c, the FT-IR spectrum (Vertex-70 FTIR spectrometer, Bruker, Germany) of the PNIPAm-g-PDMS surface shows a new absorbance peak at around $1660 \mathrm{~nm}^{-1}$, compared to the spectrum of the bare PDMS surface. This new peak can be assigned to the $\mathrm{C}=\mathrm{O}$ stretching vibration of the amide group. Another high and broad peak at $\sim 3300 \mathrm{~nm}^{-1}$ can be attributed to the $\mathrm{N}-\mathrm{H}$ stretching vibration. Both features indicate that PNIPAm has been grafted on the PDMS surface.

Surface wettability was characterized by using a contact angle interfacial tension meter (OCA Pro15, Dataphysics, Germany). Flat PDMS surfaces with and without grafted polymer were prepared using the same protocol. Images of contact angle (CA) measurements were captured at $10 \mathrm{~s}$ after a water drop was placed onto the surface. CA of $\sim 106^{\circ}$ demonstrated the hydrophobicity of the bare PDMS surface, as shown in Figure 2.1a(ii). How ever, when PNIPAm was 
grafted onto the PDMS surface, a CA of $\sim 25^{\circ}$ w as obtained (Figure 2.1b(ii)), suggesting a hydrophilic surface. SEM images (Figure 2.1a(iii) and b(iii)) showed an increase in surface roughness of PNIPAm-g-PDMS compared to bare PDMS; and the root-mean-square (RMS) roughness from AFM images (Figure 2.1 a(iv) and $b(i v)) w$ as 128.46 and $1.84 \mathrm{~nm}$ for PNIPAm-g-PDMS and bare PDMS surfaces, respectively.

UV irradiation time $w$ as investigated to determine its influence on the grafted surface wettability. As shown in Figure 2.1d, CAs on the PNIPAm-g-PDMS surfaces changed with UV irradiation time. At the UV irradiation time of $<3 \mathrm{~min}$, CA did not change significantly. For UV irradiation time of $>3 \mathrm{~min}, \mathrm{CA}$ decreased with ir radiation time, with CAs of $84 \pm 0.7,79 \pm 2,56 \pm 1,40.7 \pm 1,37.5$ \pm 0.7 , and $25 \pm 2.7^{\circ}$ obtained at the irradiation time of $3,5,10,15,20$, and $25 \mathrm{~min}$, respectively. CAs on the surfaces of bare PDMS and PDMS pretreated with benzophenone-acetone solution (PDMS with BP) did not show obvious changes with the UV irradiation time in the range of $0-25 \mathrm{~min}$.

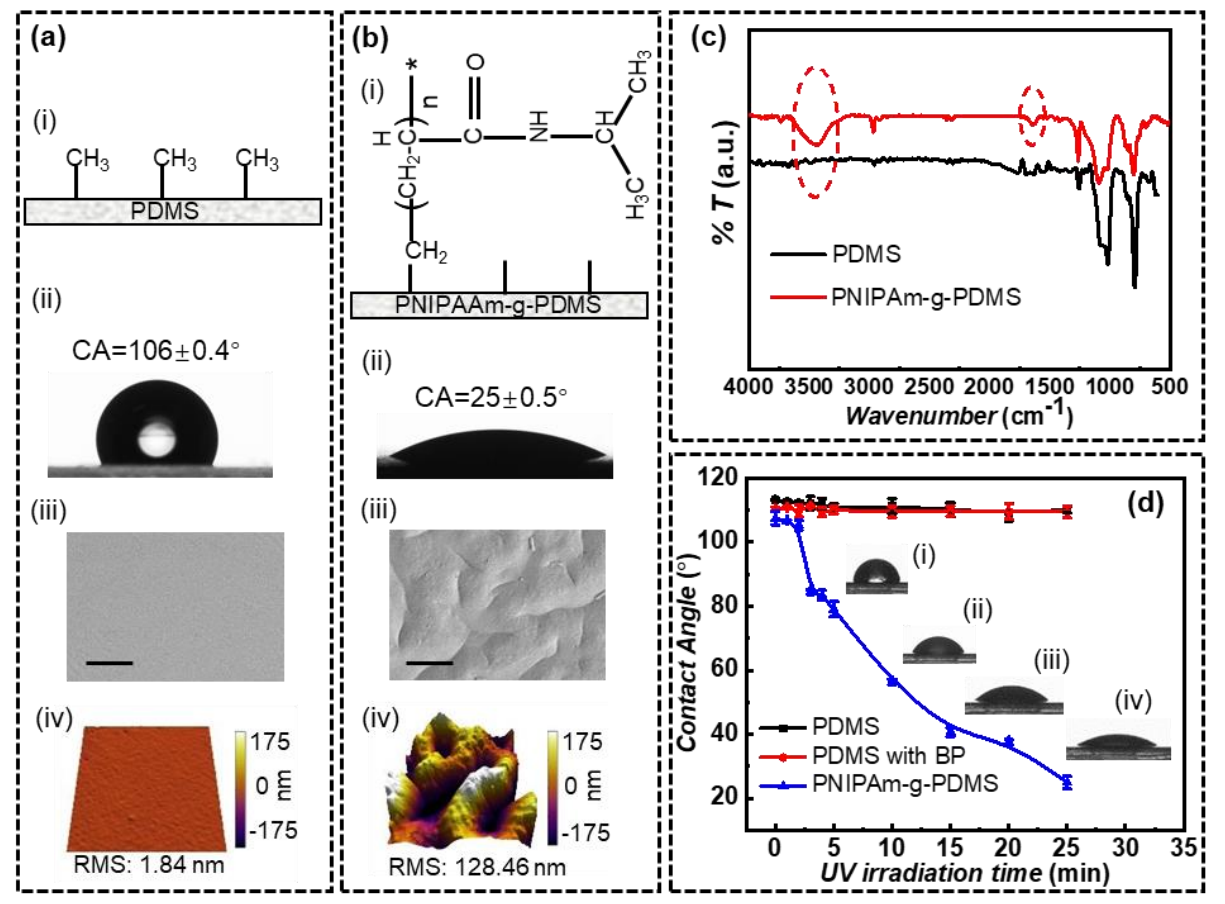

Figure 2.1. Mechanism and characterization of grafting PNIPAm on PDMS surface. (a) Bare PDMS surface: (i) chemical groups on PDMS surface, (ii) water contact angle on bare PDMS surface, (iii) SEM image of a bare PDMS surface, and (iv) AFM image of a bare 
PDMS surface (RMS = $1.84 \mathrm{~nm}$ ). (b) PNIPAm-g-PDMS surface: (i) chemical groups on PNIPAm grafted PDMS surface, (ii) water contact angle on a PNIPAm-g-PDMS surface, (iii) SEM image of a PNIPAm-g-PDMS, and (iv) AFM image of a PNIPAm-g-PDMS surface (RMS $=128.46 \mathrm{~nm}$ ). This PNIPAm-g-PDMS was prepared at $25 \mathrm{~min}$ UV irradiation. (c) FT-IR spectra of the PDMS and PNIPAm-g-PDMS. (d) Effect of UV irradiation time on water contactangles on bare PDMS, PDMS treated with benzophenone-acetone solution (PDMS with BP), and PNIPAm-g-PDMS surfaces at $25^{\circ} \mathrm{C}$, with (i), (ii), (iii) and (iv) being the PNIPAm-g-PDMS surfaces prepared at UV-irradiation time of 5, 10,15 and $25 \mathrm{~min}$, respectively. All scale bars denote $10 \mu \mathrm{m}$.

In general, a major difficulty for surface coating is its stability when exp osed to air and water, especially for polymer coatings, either due to the slow process of the movement of hydrophobic groups to the polymer surface or the low molecular w eight polymer from the bulk to the surface. ${ }^{35}$ Therefore, in this work, we have also investigated the long-term stability of PNIPAm-g-PDMS after UV irradiation times of 5,10,15,20, and 25 min by measuring the CAs after days and w eeks of storage under environmental conditions in air, with the results shown in Figure A3 (See Appendix A). Typically, the CA slightly (with about $10^{\circ}$ ) increased with storage time in several days after being prepared, and became stable after one week. When the UV irradiation time was longer than $10 \mathrm{~min}$, the surface became stable after 4 weeks of storage with CAst ypically lower than $70^{\circ}$.

\subsubsection{Thermally responsive surface w ettability of PNIPAm-g-PDMS}

A glass substrate coated with indium tin oxide (ITO) w as used as an electrical heater with a resistance of $330 \Omega$. An ITO heater was clamped with a PDMS substrate to control the PDMS surface temperature via applying a voltage for a certain duration of time. The exact temperature on a PNIPAm-g-PDMS film was measured by using a Type-T Thermocouple (Copper/Constantan), with the data collected by a data acquisition system (Keithley Series 2700, USA), as shown in Figure 2.2a(i). Patterning of the ITO heater was realized by standard photolithography. A copper w ire w as glued to the heat conductor using silver conductive epoxy. Thewater CA on the PNIPAm-g-PDMS surface was measured by repeatedly placing and removing a $2 \mu \mathrm{L}$ w ater droplet on the surface at each temperature. Figure 2.2a(ii) shows the sequential CA change on a PNIPAm-gPDMS sample surface (prepared with 25 min UV irradiation) w ith temperaturecontrolled by the ITO heater.

On a PNIPAm-g-PDMS sample surface prepared by 25 min UV initiation, the water CA varied from 24.3 to $106.1^{\circ}$ when the surface temperature increased 
from 25 to $38^{\circ} \mathrm{C}$ (Figure 2.2a). Figure 2.2b shows the water CA varying with temperature on PNIPAm-g-PDMS surfaces prepared with different UV irradiation times of 5, 10, 15, 20, and $25 \mathrm{~min}$. In contrast to the bare PDMS, all samples of PNIPAm-g-PDMS showed thermally switchable surface wettability with the corresponding CAs in the ranges of 70.8 - 101.3, 55 - 98.6, 59 - 100, 45.1 103.4, and $44.4-104^{\circ}$. At the temperature of $32^{\circ} \mathrm{C}$ (LCST of NIPAm), the CA on PNIPAm-g-PDMS surface was about $80^{\circ}$, and the surface became hydrophobic at a temperature above $34^{\circ} \mathrm{C}$. These observations show that a surface with a thermally-responsive wettability has been obtained. This surface wettability can be reversibly varied between hy drophilic and hy drophobic over a widerange of CAs.

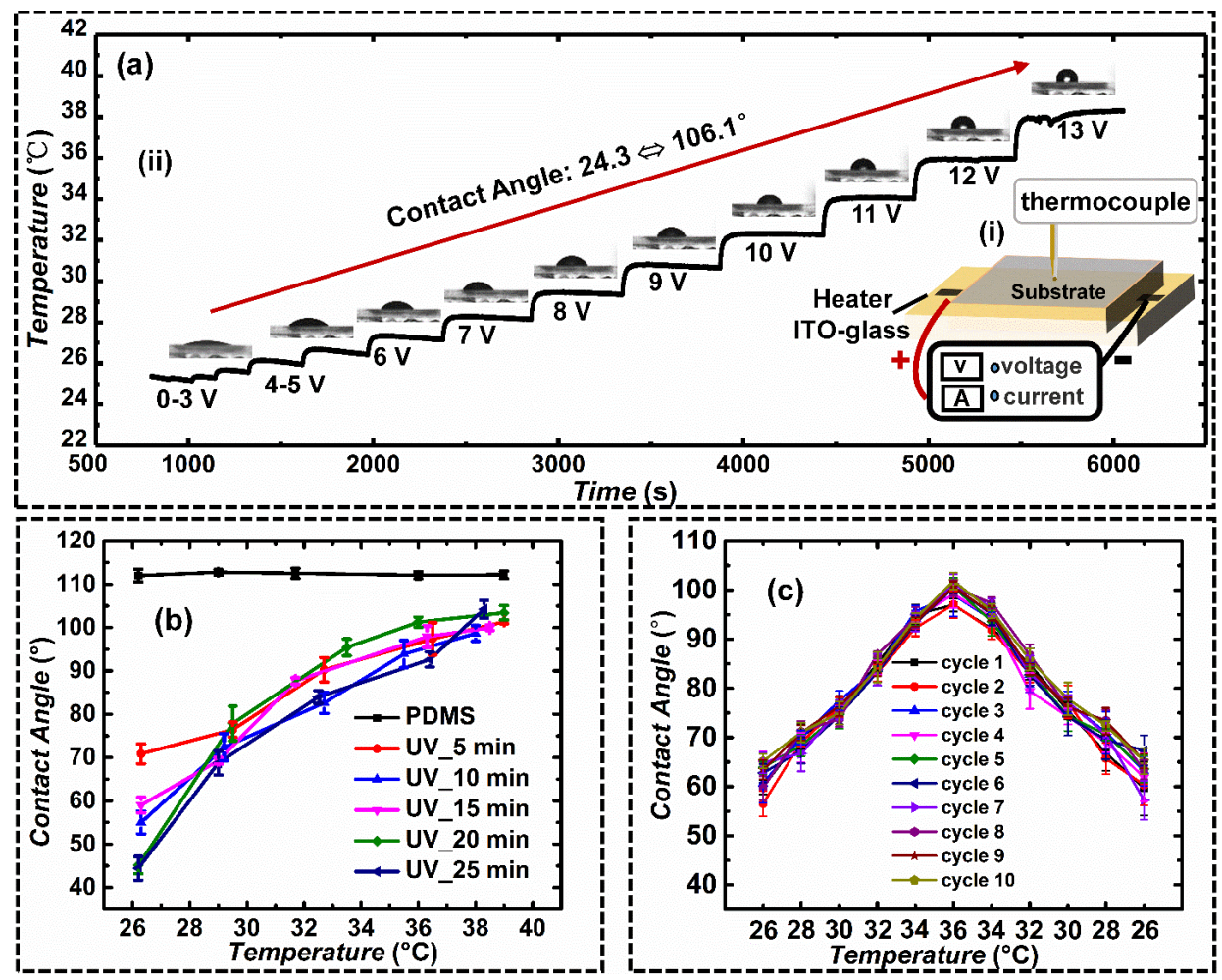

Figure 2.2. Thermo-responsive surface wettability of PNIPAm-g-PDMS. (a) Water CAs on a PNIPAm-g-PDMS surface (prepared by $25 \mathrm{~min}$ UV irradiation) changing with temperature: (i) schematic drawing of the temperature control by an ITO-heater and measurement by a thermocouple device, and (ii) PDMS surface temperature versus heating time at different applied voltages, and the water CAs on a PNIPAm-g-PDMS surface at different temperature. (b) Effect of temperature on PNIPAm-g-PDMS surface 
wettability. Samples were prepared using the same process but varying UV irradiation time of 5, 10, 15, 20, and $25 \mathrm{~min}$. (c) Reversibility and repeatability of thermo-responsive surface wettability of the PNIPAm-g-PDMS film. The error bars represent the standard deviation calculated from 5 repeated experiments for each data point.

Repeatable and reversible temperature control over the surface wettability was also investigated by heating and cooling the same sample for multiple cycles. PNIPAm-g-PDMS films were prepared under UV irradiation times of 10, 15, 20, and $25 \mathrm{~min}$. We chose a temperature range from 25 (room temperature) to $36^{\circ} \mathrm{C}$ (the PNIPAm-g-PDMS becomes hydrophobic according to Figure 2.2b). Figure 2.2c shows the surface wettability changes of a PNIPAm-g-PDMS film (10 min UV irradiation) over 10 cycles of heating and cooling. At temperature below $34^{\circ} \mathrm{C}$, the PNIPAm-g-PDMS surfaces were hydrophilic with w ater CAs of $<90^{\circ}$, while they became hydrophobic with CAs of $>90^{\circ}$ when heated above $34^{\circ} \mathrm{C}$. Experimental results show excellent reversibility in wettability control over the 10 heating-cooling cycles. The slight increase in initial CAs from $59.9 \pm 1.4$ to 65.1 $\pm 1.5^{\circ}$ after several heating-cooling cycles, may be attributed to contaminants ${ }^{54} \mathrm{or}$ the precipitation of benzophenone during the solvent evaporation in the heating process. Figure A4 (See Appendix A) show s results for PNIPAm-g-PDMS films prepared under UV irradiation time of 15,20 , and $25 \mathrm{~min}$, showing similar trends like that in Figure 2.2c. The hydrophilicity of prepared PNIPAm-g-PDMS surfaces is thus conserved for multiple heating-cooling cycles and the transition temperature from hydrophilic to hydrophobic remains at approximately $34^{\circ} \mathrm{C}$.

2.3.3 In-channel surface treatment in microfluidic devices for reversible droplet and two-phase flow types

At the micro/nano-scale, the emulsion type depends critically on the preferential wetting of the channel walls by the continuous phase, and the change of microchannel surface wettability can cause emulsion inversion. ${ }^{55}$ By using the same grafting process as for the open surface, an in-channel surface coating was realized by sequentially flowing the corresponding compounds through the selected channel areas. Figure 2.3a shows the schematic of the in-channel surface treatment process. Benzophenone solution ( $20 \mathrm{wt} \%$ in acetone) was pumped into the bare PDMS channel for 3 min to cause benzophenone to be fully absorbed in PDMS. The channel was then washed extensively with water and flushed with air to dry it. Afterward, NIPAm monomer solution containing NIPAm $(10 \%$ in water), $\mathrm{NaIO}_{4}(0.5 \mathrm{mM})$, and benzyl alcohol $(0.5 \mathrm{wt} \%)$ w as loaded into the channel. Intensive UV light $(210 \mathrm{~W}, 365 \mathrm{~nm}$, Intelliray 600, Uvitron International 
Inc., USA ) was then applied to initiate the cross-linking of the methyl groups on the PDMS and NIPAm to obtain PNIPAm-g-PDMS microfluidic channels. The channels were then flushed with DI water and kept filled with DI water by connecting the tubing to a water reservoir to avoid drying. The preferential wetting towards water or oil at different temperatures has been investigated by measuring the water in oil contact angles on a PNIPAm-g-PDMS surface, as summarized in Table A1 (See Appendix A). The grafted surface shows preferential to water and oil phases at the temperature below and beyond $32{ }^{\circ} \mathrm{C}$ (LCST of PNIPAm), respectively. This shows the possibility of controlling emulsion droplet types generated in channels by varying temperatures.

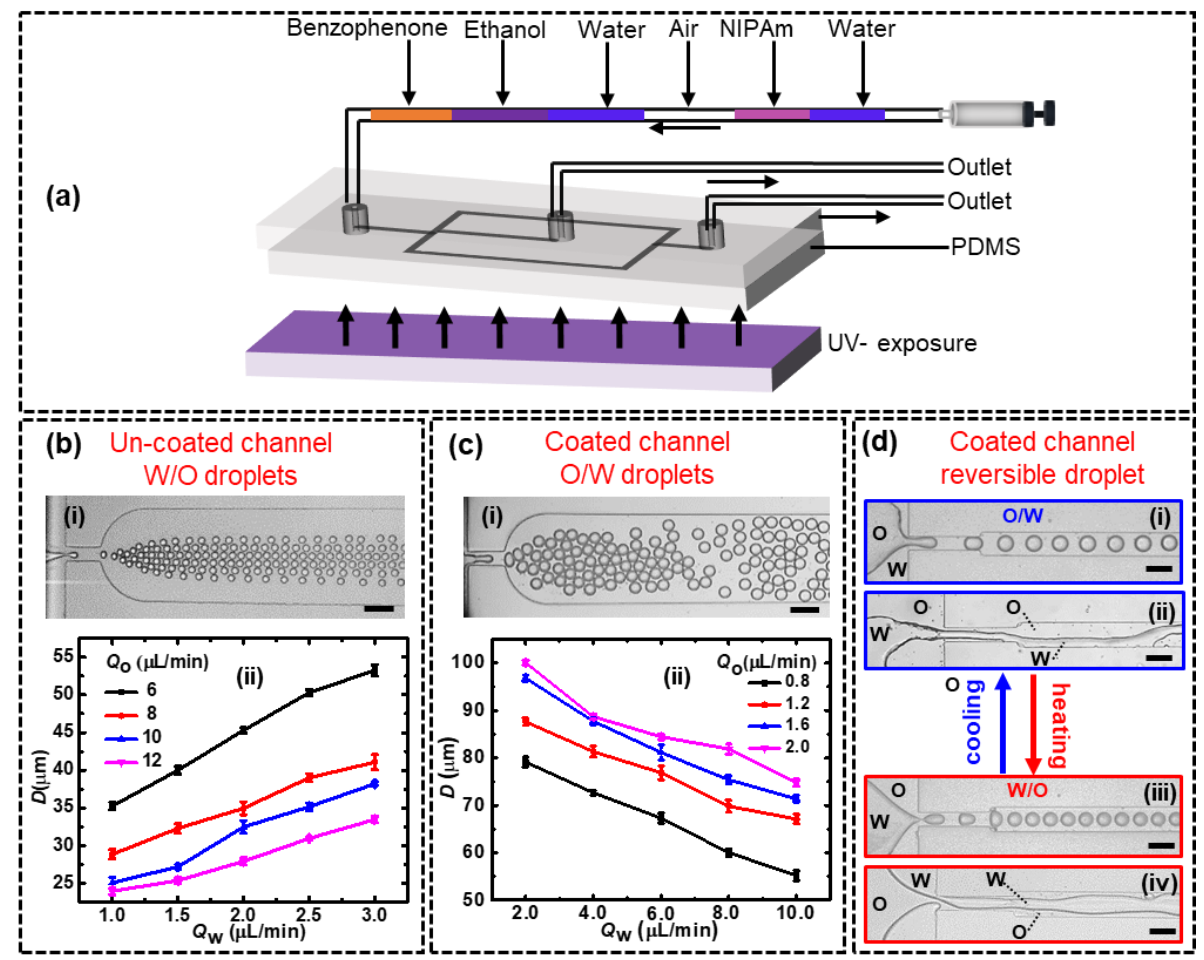

Figure 2.3. Uncoated and coated PDMS mic rofluidic chips for tunable droplet types and stratified flows via wettability control. (a) Schematic drawing of the in-channel surface treatment process. (b) W/O droplets generated in uncoated bare PDMS channels with (i) a snapshot of the droplet generation at the flow-focusing junction and (ii) the dependence of droplet diameter (D) on water phase flow rates $\left(Q_{w}\right)$ at constant oil flow rates $\left(Q_{o}\right)$. (c) $\mathrm{O} / \mathrm{W}$ droplets generated in coated PDMS channels with (i) a snapshot of the droplet generation at the flow-focusing junction and (ii) the curves of D versus $\mathrm{Q}_{\mathrm{w}}$ at constant $\mathrm{Q}_{\text {o }}$. The error bars represent the standard deviation calculated from 200 droplets for each data 
point. (d) Reversible (i) $\mathrm{O} / \mathrm{W}\left(\mathrm{Q}_{\mathrm{w}}=2.0 \mu \mathrm{L} / \mathrm{min}, \mathrm{Q}_{\mathrm{o}}=0.5 \mu \mathrm{L} / \mathrm{min}\right)$ and (iii) $\mathrm{W} / \mathrm{O}\left(\mathrm{Q}_{\mathrm{o}}=5.0\right.$ $\left.\mu \mathrm{L} / \mathrm{min}, \mathrm{Q}_{\mathrm{w}}=2.0 \mu \mathrm{L} / \mathrm{min}\right)$ droplet formation controlled by cooling $\left(25^{\circ} \mathrm{C}\right)$ and heating $\left(36{ }^{\circ} \mathrm{C}\right.$ ) the PNIPAm-g-PDMS microfluidic channels, obtained by the thermo-responsive surface, with the corresponding (ii) $\mathrm{O} / \mathrm{W}$ and (iv)W/O in-parallel flows, by using surfactants (SDS and Span 80) with opposite wettability to the channel surface. All channel depths are $60 \mu \mathrm{m}$. The scale bars denote $100 \mu \mathrm{m}$.

In general, $\mathrm{W} / \mathrm{O}$ emulsion droplets are produced in bare PDMS channels due to the hy drophobicity of the PDMS surface. As shown in Figure 2.3b, water droplets in the mineral oil solution (containing $3.0 \mathrm{wt} \% \mathrm{Span} 80$ ) were generated in a flowfocusing PDMS chip (Figure 2.3b(i)), where the droplet size w as controlled by adjusting the disperse water-phase flow rate $\left(\mathrm{Q}_{\mathrm{w}}\right)$ and the continuous oil-phase flow rate $\left(\mathrm{Q}_{\circ}\right)$. In this experiment, the values of $\mathrm{Q}_{\mathrm{w}}$ w ere fixed at 1.0, 1.5, 2.0, 2.5, and $3.0 \mu \mathrm{L} / \mathrm{min}$ with Qo being increased from 6.0 to $12.0 \mu \mathrm{L} / \mathrm{min}$. Obtained W/O emulsion droplets under different flow rate conditions are shown in Figure $2.3 \mathrm{~b}$ (ii), suggesting that the generated droplet diameter (D) increases with the increase of $Q_{w}$ and decreases with the increase of $Q_{o}$. Monodispersed water droplets are obtained with diameters ranging from 23 to $54 \mu \mathrm{m}$ and corresponding relative standard deviations (RSDs) less than $3.0 \%$.

When the hydrophobic PDMS surface was grafted with a layer of PNIPAm, a hydrophilic surface was obtained (Figure 2.1b). As a result, the water phase preferentially wetted the channel surface instead of oil, and consequently, the formation of oil droplets in the water phase $w$ as achieved, as demonstrated in Figure 2.3c. Mineral oil droplets were formed in an aqueous solution containing $3.0 \mathrm{wt} \%$ sodium dodecyl sulfate (SDS) in PNIPAm grafted PDMS chips (Figure 2.3c(i)). Generated droplet size could be tuned by varying the continuous water $\left(\mathrm{Q}_{\mathrm{w}}\right)$ and dispersed oil $\left(\mathrm{Q}_{\mathrm{o}}\right)$ flow rates in the same device, being increase with the increase of $Q_{o}$ and decrease $w$ ith the increase of $Q_{w}$, as shown in Figure 2.3c(ii). In this experiment, $Q_{o} w$ as fixed at $0.8,1.2,1.6$, and $2.0 \mu \mathrm{L} / \mathrm{min}$ with $\mathrm{Q}_{w}$ increased from 2.0 to $10.0 \mu \mathrm{L} / \mathrm{min}$. The obtained oil droplet dia meter was in the range of 55 - $101 \mu \mathrm{m}$ with RSDs of $<2 \%$. To evaluate the coating stability, the same device was reused several times over one month. Similar O/W droplet generation processes and flow regimes were obtained w ithout obvious changes.

The thermo-responsive wettability in the microfluidic channels $\mathrm{w}$ as then evaluated by heating-cooling cycles. An ITO heater was placed underneath the microfluidic device to control the temperature via the applied voltage. As shown in Figure 2.2b, the hydrophilic PNIPAm-g-PDMS surface could be efficiently 
changed to hydrophobic by heating above $34^{\circ} \mathrm{C}$. In the experiment, the surface w as heated up to $\sim 36{ }^{\circ} \mathrm{C}$ to ensure a hydrophobic channel surface. At the room temperature of $\sim 25{ }^{\circ} \mathrm{C}$, the PNIPAm grafted PDMS channels behaved hydrophilic, making the w ater phase acting as the continuous phase. As shown in Figure 2.3d(i), silicon oil droplets were produced in an aqueous solution containing $3.0 \mathrm{wt} \%$ sodium dodecyl sulfate (SDS) as the surfactant. Also when using silicon oil containing $2.0 \mathrm{wt} \%$ Span 80 as the continuous phase (a more hy drophobic surfactant), $w$ ater droplets w ere not formed which can be ascribed to the fact that the oil phase is disfavored by the channel w alls (Figure 2.3d(ii)). Instead, as we expect, due to the competition between the interfacial preference of forming W/O curvature caused by the Span 80 surfactant and the solid surface w ettability to the $w$ ater phase, an oil-in-water in-parallel flow was still obtained but without droplet formation. When the channel is heated up to $36^{\circ} \mathrm{C}$, the coated channel surface becomes hydrophobic and the oil phase wets the channel surface. As a result, water droplets in silicon oil containing 2.0\% Span 80 were formed (Figure 2.3d(iii)). Due to the same reason as described above, in the hydrophobic channel at high temperature, water-in-oil in-parallel flow was observed as shown in Figure 2.3d(iv). In summary, the continuous and dispersed phases could be changed at will by thermally switching the surface w ettability.

2.3.4 Surface w ettability determined stable and unstable droplet formation with the same fluidic compositions in the same microfluidic device

As show $n$ above, stable $\mathrm{O} / \mathrm{W}$ and $\mathrm{W} / \mathrm{O}$ droplets could be stably generated in thermally switched hydrophilic and hydrophobic channels, respectively, by applying suitable hydrophilic and hy drophobic surfactants in the w ater and oil phases. However, when a two-phase fluidic system is used of a constant composition that can generate both $\mathrm{O} / \mathrm{W}$ and $\mathrm{W} / \mathrm{O}$ droplets, the microfluidic channel surface wettability becomes the key factor controlling the droplet creation processes and thus the emulsion types. Thus, a microfluidic channel with tunable surface wettability can help to find out the boundaries of the regimes where $\mathrm{O} / \mathrm{W}$ and $\mathrm{W} / \mathrm{O}$ droplets are created, as w ell as the regimes where stable and unstable droplet formation processes occur. To investigate this possibility, w e chose a two-phase fluidic system $w$ ith a hydrophilic surfactant in w ater and a hydrophobic surfactant in oil. An aqueous solution containing 4.0 $\mathrm{w} t \%$ PVA (surfactant) and $30 \mathrm{w} t \%$ glycerol was used as the aqueous phase, and HFE - 7500 containing $2.0 \mathrm{wt} \%$ PFPE- PEG600 (surfactant) $\mathrm{w}$ as applied as the oil phase. From this fluidic system, both $\mathrm{O} / \mathrm{W}$ and $\mathrm{W} / \mathrm{O}$ droplets could be generated 
by using conventional mechanical agitation. In this work, we could dynamically adjust the surface wettability by heating and cooling the microfluidic devices with the PNIPAm-g-PDMS channel surface. As a result, stable or unstable O/W, and stable or unstable W/O droplets could be obtained at choice in a single microfluidic device by regulating the temperature, results are shown in Figure 2.4. At room temperature, the w ater phase served as the continuous phase since the PNIPAm coated channel wall is hydrophilic (with a w ater contact angle of $56.3^{\circ}$ ), and $\mathrm{O} / \mathrm{W}$ emulsion droplets were obtained at a large range of oil and water flow rates. When the oil (disperse phase) flow rate was too high toform droplets, the stratified flow $w$ as obtained $w$ ith the $w$ ater phase still wetting the channel w alls. How ever, when heating up to $32.5^{\circ} \mathrm{C}$, the w ater CA changes to $842^{\circ}$ and the oil phase started to wet the channel wall and hence became the continuous phase. As a result, W/O emulsion droplets w ere obtained. Moreover, in this case, we have observed stratified flow when the water flow rate increased above a threshold value, with the oil phase still wetting the channel walls. In this way, it proves that the surface wettability determines emulsion types generated inside the microfluidic device no matter how inner and outer phases are introduced. Such a surface-wettability-induced dynamic reversible emulsion process will be highly useful for studying the phase inversion mechanism, which has applications in the food and the cosmetics industry where phase inversion is necessary or instead needs to be avoided. ${ }^{11,12}$

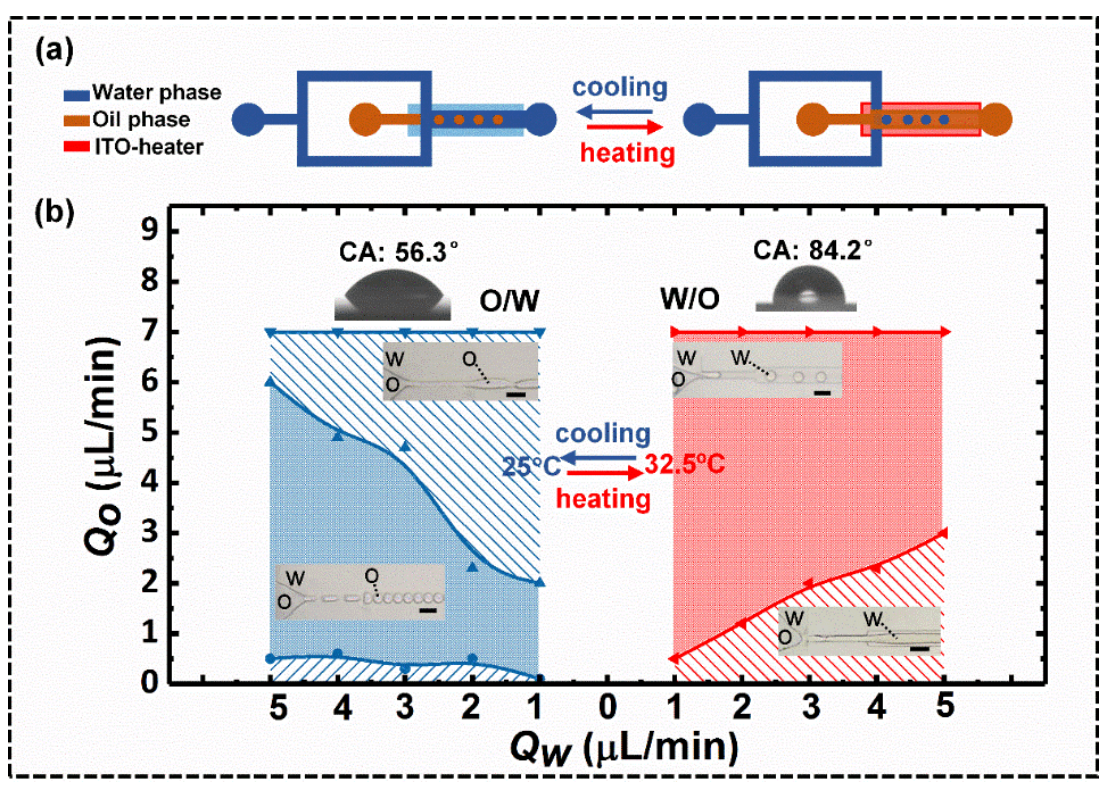


Figure 2.4. Reversible tuning $\mathrm{O} / \mathrm{W}$ and $\mathrm{W} / \mathrm{O}$ at droplet and stratified flows by thermo responsive surface wettability in one microfluidic device using the same fluidic system. (a) Diagrammatic drawing of dynamically reversible emulsion droplet generation. (b) Stable and unstable droplet formation in a heating and cooling process. Blue and red areas are $\mathrm{O} / \mathrm{W}$ and $\mathrm{W} / \mathrm{O}$ flow ty pes, being reversible in a cooling and heating cycle. CAs of $56.3^{\circ}$ and $84.2^{\circ}$ represent hydrophilic and hydrophobic surface wettability at the temperature of 25.0 and $32.5^{\circ} \mathrm{C}$, respectively. The microfluidic channels were coated with PNIPAm under UV irradiation time of $10 \mathrm{~min}$. The water and oil phases were aqueous solution containing $4.0 \mathrm{wt} \%$ PVA (surfactant) and $30 \mathrm{wt} \%$ glycerol, and HFE -7500 containing 2.0 wt $\%$ PFPE-PEG600 (surfactant). All scale bars denote $50 \mu \mathrm{m}$.

2.3.5 Controllable double emulsion droplet preparation in microfluidic devices by local temperature control

Double emulsions of either $\mathrm{W} / \mathrm{O} / \mathrm{W}$ and $\mathrm{O} / \mathrm{W} / \mathrm{O}$ ty pes can encapsulate various contents as cores (inner phase) via forming shells (middle phase) to form coreshell structures and have found a variety of applications including microcapsule fabrication, ${ }^{56}$ vesicle preparation, ${ }^{57}$ drug delivery, ${ }^{58,59}$ chemical synthesis, ${ }^{60}$ and single-cell screening. ${ }^{61}$ Controlled fabrication of multiple emulsion droplets using microfluidics has been well developed in recent years. Generation of double emulsion droplets has been reported by using microfluidic devices of assembled glass capillaries ${ }^{60,61}$ or selectively modified microfluidic channels. ${ }^{23,62-}$ ${ }^{64}$ Fixed emulsion types of either $\mathrm{W} / \mathrm{O} / \mathrm{W}$ or $\mathrm{O} / \mathrm{W} / \mathrm{O}$ could be produced in those devices once the microfluidic channels w ere prepared. Hereby, we demonstrate that the necessary surface wettability changes can also be obtained in a reversible and switchable manner in a single device by PNIPAm grafted surface and temperature control. For this purpose, microfluidic devices with two sections of flow-focusing junctions were selectively modified. Local non-modification of PDMS channels $w$ as enabled by selectively filling the channel section with air to block the BP solution, and thus preventing PNIPAm grafting to keep its hydrophobicity. As demonstrated in Figures A5a(i) and b(i) (See Appendix A), PDMS microfluidic devices were selectively modified to obtain opposite wettability at the two consecutive flow-focusing-device (FFD) sections. Figure A5 show s the cor responding formation of single and double emulsion droplets. The sequential untreated-treated and treated-untreated PDMS FFDs could produce $\mathrm{W} / \mathrm{O} / \mathrm{W}$ and $\mathrm{O} / \mathrm{W} / \mathrm{O}$ droplets, respectively. Here, the high contrast in surface wettability of the PDMS $\left(\mathrm{CA}=106^{\circ}\right)$ and PNIPAm-g-PDMS $\left(\mathrm{CA}=25^{\circ}\right)$ is used for stably creating double emulsions. 
The key advantage of the PNIPAm material is its thermo-responsive property. Therefore, we investigated in-channel local surface w ettability control by locally heating part of the microfluidic channels, as demonstrated in Figure 2.5. The two FFD sections were first treated to be hydrophilic by grafting a PNIPAm layer. Under each FFDsection, an ITO heater strip was placed to selectively control its surface temperature via an applied voltage, as shown in Figures 2.5a(i) and b(i). In this way, the surface wettability of the two sections of FFD could be selectively manipulated with the same or opposite wettability with a water contact angle difference of about $80^{\circ}$.

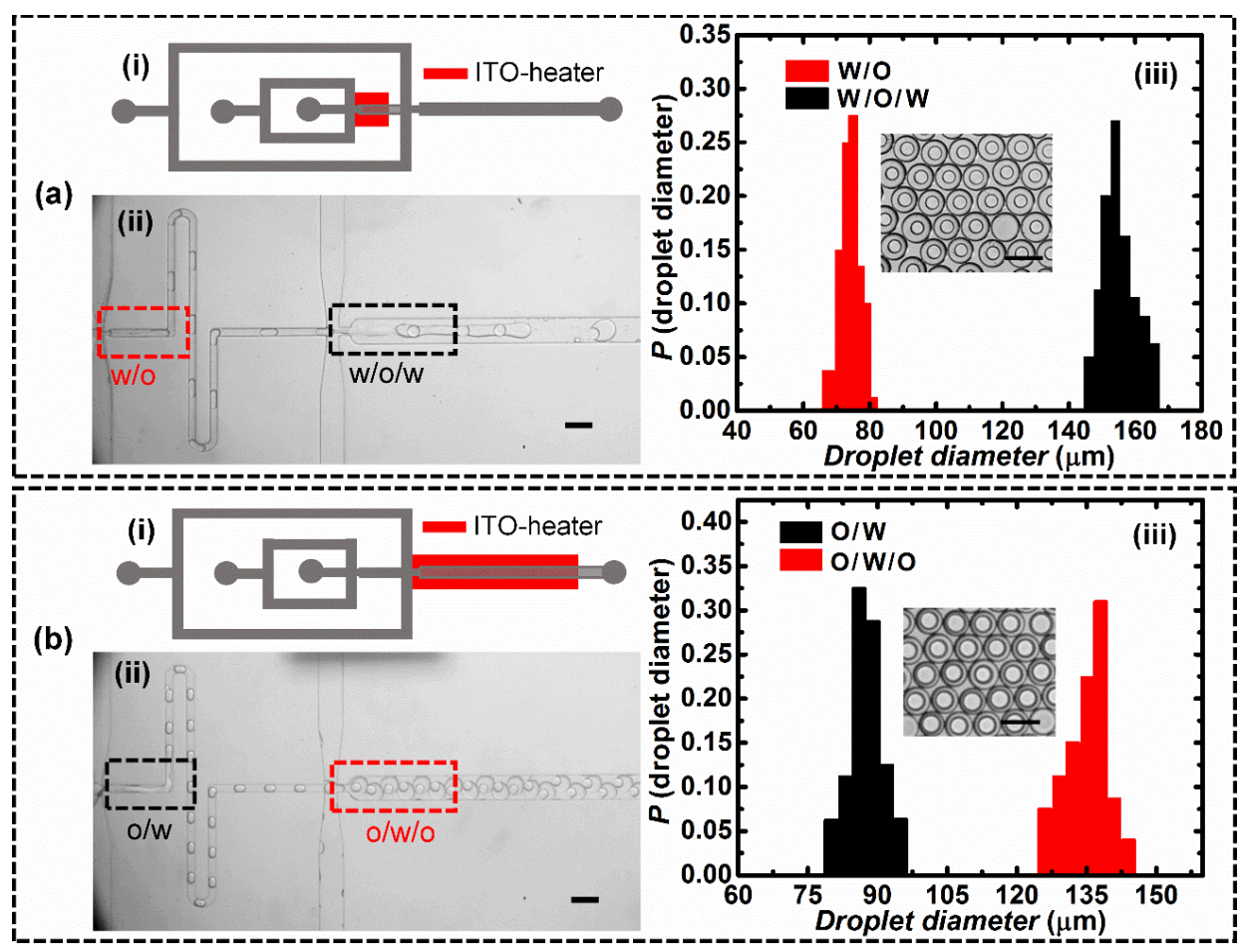

Figure 2.5. Local surface wettability control via temperature, and corresponding double emulsion droplets formation in the same microfluidic device with PNIPAm-g-PDMS surface. (a) Local heating at the first FFD section for producing W/O/W double emulsion droplets: (i) schematic drawing of selectively heating the first FFD section, (ii) formation of $\mathrm{W} / \mathrm{O}$ and $\mathrm{W} / \mathrm{O} / \mathrm{W}$ droplets at the first and second FFD junctions, and (iii) collected droplets and droplet size distribution. (b) Local heating at the second FFD section for producing $\mathrm{O} / \mathrm{W} / \mathrm{O}$ double emulsion droplets: (i) schematic drawing of selectively heating the secondFFD section, (ii) formation of $/ \mathrm{W}$ and $\mathrm{O} / \mathrm{W} / \mathrm{O}$ droplets at the first and second FFD junctions, and (iii) collected droplets and droplet size distribution. All scale bars 
denote $200 \mu \mathrm{m}$. The droplet diameter distribution was calculated with a total droplet number of 200.

To form W/O/W double emulsion droplets, the first (left) FFDsection should be hydrophobic while the second (right) FFD section should be hydrophilic. The PNIPAm-g-PDMS surface is hydrophilic at a temperature of $\sim 25^{\circ} \mathrm{C}$. When the first FFD section was heated up to $36^{\circ} \mathrm{C}$ while keeping the second FFD section at $\sim 25^{\circ} \mathrm{C}$, the first and second FFD sections became hydrophobic and hydrophilic, respectively. Indeed, $\mathrm{W} / \mathrm{O} / \mathrm{W}$ double emulsion droplets were obtained, as presented in Figure 2.5a(ii). The inner, middle and outer fluidic phases were DI w ater, FC-40 containing $2.0 \mathrm{wt} \%$ PFPE-PEG600 as the surfactant, and an aqueous solution containing $2 \mathrm{wt} \%$ PVA and $15 \mathrm{wt} \%$ glycerol, respectively, with the corresponding flow rates of $0.3,1.8$, and $14 \mu \mathrm{L} / \mathrm{min}$. On the other hand, $\mathrm{O} / \mathrm{W} / \mathrm{O}$ double emulsion droplets were successfully generated in thesame device by just locally heating the second FFD section, as shown in Figure 2.5b(ii). The inner, middle, and outer fluidic phases were HFE-7500, an aqueous solution containing $2.0 \mathrm{wt} \%$ PVA and $15.0 \mathrm{wt} \%$ glycerol, and HFE-7500 containing $2.0 \mathrm{wt} \%$ PFPEPEG600 as the surfactant, respectively, $w$ ith the cor responding flow rates of 0.5 , 1.5 , and $4.0 \mu \mathrm{L} / \mathrm{min}$. Collected droplets were analyzed using Image J software, as presented in Figures 2.5a(iii) and b(iii), both the single emulsion droplets produced at the first FFD junction and the double emulsion droplets generated at the second FFD junction show good monodispersity with a relative standard deviation of $<7 \%$. Higher enwrapping efficiency (the portion of double emulsion droplets in all generated droplets) $\mathrm{w}$ as obtained by adjusting the flow rates of the three phases.

2.3.6 Synthesis of core-shell microcapsules via double emulsion droplets as templates

To demonstrate the usability and reliability of the thermo-responsive surface wettability of the PNIPAm-g-PDMS, preparation of core-shell microcapsules in locally heated PNIPAm-g-PDMS chips was investigated. To produce core-shell microcapsules, double emulsion droplets containing an inner liquid phase (core) and middle liquid phase (shell) in the outer liquid phase are prepared. In this w ork, we chose the aqueous solution containing $10.0 \mathrm{wt} \%$ glycol, the ethoxylated trimethylolpropane triacrylate (ETPTA) with 2-dimethoxy-2-phenyl acetophenone, and the aqueous solution containing $4.0 \mathrm{wt} \% \mathrm{PVA}$ and $30.0 \mathrm{wt} \%$ glycerol, as inner, middle, and outer phases, respectively. Monodispersed double emulsion droplets were obtained at the inner, middle, and outer flow rate ranges 
of $0.035-0.4,0.45,8.0 \mu \mathrm{L} / \mathrm{min}$, respectively. Collected droplets were then exposed to UV light $(210 \mathrm{~W}, 365 \mathrm{~nm}$, Intelliray 600, Uvitron International Inc., USA) for 20 $s$ to polymerize the middle ETPTA phase to form a hard shell. Microcapsules with different shell thicknesses were obtained by varying the flow rate of the inner phase. Figure 2.6 shows the microscopic images of the double emulsion droplets and SEM images of the polymerized core-shell microcapsules with ETPTA shells at various thicknesses. The double emulsion droplets were obtained at the inner flow rates of $0.08,0.15,0.28,0.35$, and $0.38 \mu \mathrm{L} / \mathrm{min}$ while keeping the middle and outer flow rates at 0.45 and $8.0 \mu \mathrm{L} / \mathrm{min}$. Before SEM analysis, the microcapsules w ere ruptured between two glass slides to view the cross-sectional area. A $15 \mathrm{~nm}$ platinum layer was deposited onto the microcapsule surface before putting intothe chamber of SEM. The microcapsule size and shell thickness in the range of $30-150 \mu \mathrm{m}$ and $1-40 \mu \mathrm{m}$ could be created by precisely tuning the fluidic properties and flow rates. Such microcapsules could be applied as micro-containers for encapsulating active agents for the preparation of fragrance retention pow der, ${ }^{65}$ osmotic pressure triggered cavitation, ${ }^{66}$ photonic crystals, ${ }^{67}$ and so on. Moreover, since the wettability of the tw o FFDjunctions could both be tuned between hydrophilic and hydrophobic to obtain high wettability contrast, various types of microcapsule materials could be synthesized using such a device, which would explore materials with more functionalities, and thus expand their application fields.

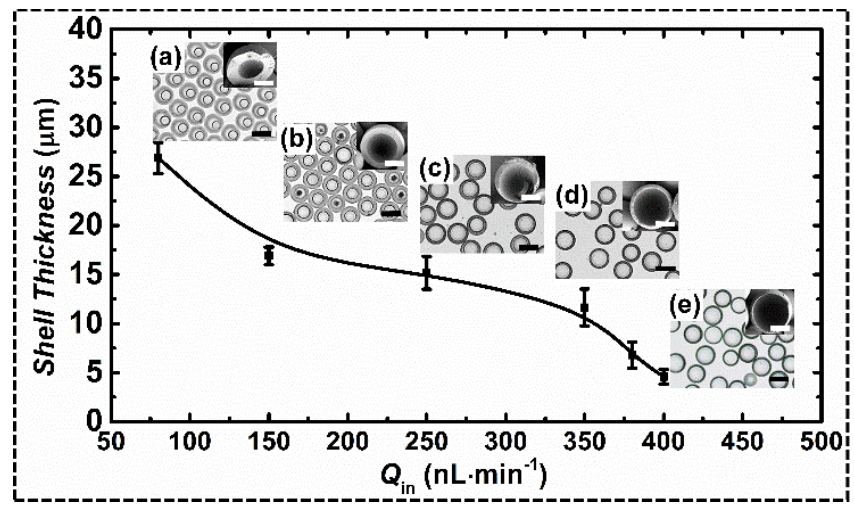

Figure 2.6. Controllable generation of double emulsion droplets for fabricating core-shell microcapsules with different shell thickness. (a), (b), (c), (d) and (e) are microsco pic images and SEM images of double emulsion droplets and core-shell microcapsules prepared at the inner flow rates of $0.08,0.15,0.28,0.35$ and $0.38 \mu \mathrm{L} / \mathrm{min}$, with the constant middle and outer phase flow rates of 0.45 and $8.0 \mu \mathrm{L} / \mathrm{min}$. The white scale bars denote $40 \mu \mathrm{m}$, and 
black scale bars denote $100 \mu \mathrm{m}$. The standard deviation was calculated from 100 core-shell capsules for each data point.

\subsection{CONCLUSION}

In this work, a surface coating method based on photo-grafting of the thermoresponsive polymer PNIPAm onto PDMS has been investigated and applied in microfluidic devices. Bare hydrophobic PDMS surfaces became stably hydrophilic when grafted with PNIPAm. A highly sensitive and reversible thermo-responsive wettability change of $7.4^{\circ} /{ }^{\circ} \mathrm{C}$ w as achieved on the surfaces with the water CA changing between 25 and $106^{\circ}$ when varying the PNIPAm-gPDMS surface temperature between 25 and $38^{\circ} \mathrm{C}$. The surface modification, as well as the thermal switching, were found to be long-term stable under environmental conditions. A dynamically reversible generation of $\mathrm{O} / \mathrm{W}$ and $\mathrm{W} / \mathrm{O}$ emulsions was achieved via heating and cooling the same microfluidic device with PNIPAm grafted walls. Such a high wettability contrast with a switchable CA difference of $\sim 80^{\circ}$ brings high controllability over the single and double emulsion droplet for mation using various fluidic compositions. Both $\mathrm{O} / \mathrm{W} / \mathrm{O}$ and $\mathrm{W} / \mathrm{O} / \mathrm{W}$ emulsion droplets can be generated either via selectively grafting part of PDMS channels or selectively heating the overall grafted microfluidic channels as designed, according to the opposite wettability of PDMS and PNIPAm-gPDMS surfaces, and the thermo-responsive w ettability of the PNIPAm-g-PDMS surface. Dynamic tuning of the channel surface wettability enabled us, for the first time, to establish the regimes of $\mathrm{O} / \mathrm{W}$ droplet flow, $\mathrm{O} / \mathrm{W}$ stratified flow, $\mathrm{W} / \mathrm{O}$ droplet flow, and W/O stratified flow, for the same two-phase fluidic system in the same microfluidic device. Core-shell microcapsules containing a fluidicinner core and a hard shell were also synthesized via the double emulsion droplet process, with a tunable core and shell thickness. Overall, we demonstrated a facile and rapid surface modification to prepare long-term stable hydrophilic PDMS surfaces with thermal wettability switching between hydrophilic and hydrophobic states. This method may serve as a useful platform to study dynamical processes of reversible multiphase micro/nano-fluidic phenomena on surfaces or in confined micro-spaces.

\subsection{REFERENCES}

1. Rajaei-Sharifabadi, H.; Greene, E.; Piekarski, A.; Falcon, D.; Ellestad, L; Donoghue, A.; Bottje, W.;Porter, T.; Liang, Y.; Dridi, S. Surface Wetting Strategy Prevents Acute Heat Exposure-Induced Alterations of 
Hy pothalamicStress- and Metabolic-Related Genes in Broiler Chickens. J. Anim.Sci. 2017, 95 (3), 1132-1143.

2. Charles-Orszag, A.; Tsai, F.-C.; Bonazzi, D.; Manriquez, V.; Sachse, M.; Mallet, A.; Salles, A.; Melican, K.; Staneva, R.; Bertin, A.; Millien C.. Adhesion to Nanofibers Drives Cell Membrane Remodeling through One-Dimensional Wetting. Nat. Commun. 2018, 9 (1), 4450.

3. Meskini-Vishkaee, F.; Mohammadi, M. H.; Neyshabouri, M. R. Revisiting the Wet and Dry Ends of Soil Integral Water Capacity Using Soil and Plant Properties. Soil Res. 2018, 56 (4),331-345.

4. Du X, Wang J, Cui H, Zhao Q, Chen H, He L, Wang Y. Breath-Taking Patterns: Discontinuous Hydrophilic Regions for Photonic Crystal Beads Assembly and Patterns Revisualization. ACS applied materials \& interfaces. 2017, 9(43):38117-38124.

5. Sigal, G. B.; Mrksich, M.; Whitesides, G. M. Effect of Surface Wettability on the Adsorption of Proteins and Detergents. J. Am. Chem. Soc. 1998, 120 (14), 3464-3473.

6. $\quad$ Bi, C.; Wang, Q.; Shao, Y.; Yuan, Y.; Xiao, Z.; Huang, J. Non-Wetting Surface-Driven High-Aspect-Ratio Crystalline Grain Growth for Efficient Hybrid PerovskiteSolar Cells. Nat. Commun. 2015, 6 (1), 7747.

7. Tetsuka, H.;Matsui, T. Non-IonicFluorosurfactant Improves Wettability of Nitrogen-Functionalized Graphene Quantum Dots for Integration with Optoelectronic Devices. Chem. Lett. 2018, 47 (7), 850-852.

8. Chappat, M. Some Applications of Emulsions. Colloids Surfaces A Physicochem. Eng. Asp. 1994, 91, 57-77.

9. Guzey, D.; McClements, D. J. Formation, Stability and Properties of Multilayer Emulsions for Application in the Food Industry. Adv. Colloid Interface Sci. 2006, 128, 227-248.

10. Koroleva, M. Y.; Nagovitsina, T.Y.; Bidanov, D. A.; Gorbachevski, O. S.; Yurtov, E. V. Nano- and Microcapsules as Drug-Delivery Systems. Resour. Technol. 2016, 2, 233-239.

11. Thakur, R. K.; Villette, C.; Aubry, J. M.; Delaplace, G. Dynamic Emulsification and Catastrophic Phase Inversion of Lecithin-Based Emulsions. Colloids Surfaces A Physicochem. Eng. Asp. 2008, 315 (1), 285-293.

12. Perazzo, A.; Preziosi, V.; Guido, S. Phase Inversion Emulsification: Current Understanding and Applications. Adv. Colloid Interface Sci. 2015, 222, 581-599.

13. Teh, S. Y.; Lin, R.;Hung, L. H.; Lee, A.P. Droplet Microfluidics. LabChip $2008,8(2), 198-220$. 
14. Kelly, B. T.; Baret, J.-C.; Taly, V.; Griffiths, A.D. Miniaturizing Chemistry and Biology in Microdroplets. Chem. Commun. 2007, 38 (18), 1773-1778.

15. Liu, G.; Wang, X.; Wang, K.; Tostado, C. P.; Luo, G. Effect of Surface Wettability on Internal Velocity Profile during Droplet Formation Process in MicrofluidicDevices. Int. J. Multiph. Flow 2016, 80, 188-193.

16. Wehking, J. D.; Gabany, M.; Chew, L.; Kumar, R. Effects of Viscosity, Interfacial Tension, and Flow Geometry on Droplet Formation in a Microfluidic T-Junction. Microfluid. Nanofluidics 2014,16 (3), 441-453.

17. Shui, L.; Pennathur, S.; Eijkel, J. C. T.; Van Den Berg, A. Multiphase Flow in Lab on Chip Devices: A RealTool for the Future? Lab Chip2008, 8 (7), 1010-1014.

18. Derzsi, L.; Jankow ski, P.; Lisow ski, W.; Garstecki, P. Hydrophilic Polycarbonate for Generation of Oil in Water Emulsions in Microfluidic Devices. Lab Chip 2011, 11 (6), 1151-1156.

19. Okushima, S.; Nisisako, T.; Torii, T.; Higuchi, T. Controlled Production of Monodisperse Double Emulsions by Two-Step Droplet Breakup in Microfluidic Devices. Langmuir 2004, 20 (23), 9905-9908.

20. Wu, N.; Oakeshott, J. G.; Easton, C. J.; Peat, T. S.; Surjadi, R.; Zhu, Y. A Double-Emulsion Microfluidic Platform for in Vitro Green Fluorescent Protein Expression. J. Micromechanics Microengineering 2011, 21 (5), 054032.

21. Carvalho, R. R.;Pujari, S.P.; Lange, S.C.;Sen, R.; Vrouwe, E. X.;Zuilhof, $\mathrm{H}$. Local Light-Induced Modification of the Inside of Microfluidic Glass Chips. Langmuir 2016, 32 (10), 2389-2398.

22. Bashir, S.; Bashir, M.; Casadevall i Solvas, X.; Rees, J. M.; Zimmerman, W. B. Hy drophilic Surface Modification of PDMS Microchannel for O/W and W/O/W Emulsions. Micromachines 2015, 6 (10), 1445-1458.

23. Abate, A. R.; Krummel, A. T.; Lee, D.; Marquez, M.;Holtze, C.; Weitz, D. A. Photoreactive Coating for High-Contrast Spatial Patterning of Microfluidic Device Wettability. Lab Chip 2008, 8 (12), 2157-2160.

24. Nisisako, T.; Okushima, S.; Torii, T. Controlled Formulation of Monodisperse Double Emulsions in a Multiple-Phase Microfluidic System. Soft Matter 2005, 1 (1), 23-27.

25. Pannacci, N.; Bruus, H.; Bartolo, D.; Etchart, I.; Lockhart, T.; Hennequin, Y.; Willaime, H.; Tabeling, P. Equilibrium and Nonequilibrium States in Microfluidic Double Emulsions. Phys. Rev. Lett. 2008, 101(16), 164502.

26. Bhushan, B. Springer Handbook of Nanotechnology; Bhushan, B., Ed.; Springer Berlin Heidelberg: Ber lin, Heidelberg, 2004, 487-536. 
27. McDonald, J. C.; Duffy, D. C.; Anderson, J. R.; Chiu, D. T.; Wu, H.; Schueller, O. J.; Whitesides, G. M. Fabrication of Microfluidic Systems in Poly(Dimethylsiloxane). Electrophoresis 2000, 21 (1), 27-40.

28. Zhou, J.; Ellis, A. V.; Voelcker, N. H. Recent Developments in PDMS Surface Modification for Microfluidic Devices. Electrophoresis 2010, 31 (1), 2-16.

29. Bauer, W. A. C.; Fischlechner, M.; Abell, C.; Huck, W. T. S. Hydrophilic PDMS Microchannels for High-Throughput Formation of Oil-in-Water Microdroplets and Water-in-Oil-in-Water Double Emulsions. Lab Chip 2010, 10 (14), 1814-1819.

30. Fritz, J. L.; Owen, M. J. Hydrophobic Recovery of Plasma-Treated Polydimethylsiloxane. J. Adhes. 1995, 54 (1-4),33-45.

31. Trantidou, T.; Elani, Y.; Parsons, E.; Ces, O. Hydrophilic Surface Modification of PDMS for Droplet Microfluidics Using a Simple, Quick, and Robust Method via PVA Deposition. Microsystems Nanoeng. 2017, 3, 16091 .

32. Roman, G. T.;Hlaus, T.; Bass, K. J.; Seelhammer, T. G.; Culbertson, C. T. Sol-Gel Modified Poly(Dimethylsiloxane) Microfluidic Devices with High Electroosmotic Mobilities and Hydrophilic Channel Wall Characteristics. Anal. Chem. 2005, 77 (5), 1414-1422.

33. Abate, A. R.; Lee, D.; Do, T.; Holtze, C.; Weitz, D. A. Glass Coating for PDMS Microfluidic Channels by Sol-gel Methods. Lab Chip 2008, 8 (4), 516-518.

34. Choi, C. H.; Lee, H.; Weitz, D. A. Rapid Patterning of PDMS Microfluidic Device Wettability Using Sy ringe-Vacuum-Induced Segmented Flow in Nonplanar Geometry. ACS Appl. Mater. Interfaces 2018, 10 (4), 31703174.

35. Hu, S.; Ren, X.; Bachman, M.;Sims, C.; Li, G. Surface Modification of Poly (Dimethylsiloxane) Microfluidic Devices by Ultraviolet Polymer Grafting. Anal. Chem. 2002, 74 (16), 4117-4123.

36. Schneider, M. H.; Willaime, H.; Tran, Y.; Rezgui, F.; Tabeling, P. Wettability Patterning by UV-Initiated Graft Polymerization of Poly(Acrylic Acid) in Closed Microfluidic Systems of Complex Geometry. Anal. Chem. 2010, 82 (21), 8848-8855.

37. Wang, Y.; Lai, H. H.; Bachman, M.;Sims, C. E.; Li, G. P.; Allbritton, N. L. Covalent Micropatterning of Poly(Dimethylsiloxane) by Photografting through a Mask. Anal. Chem. 2005, 77 (23), 7539-7546.

38. Hu, S.; Ren, X.; Bachman, M.; Sims, C. E.; Li, G. P.; Allbritton, N. L. Surface-Directed, Graft Polymerization within Microfluidic Channels. Anal. Chem. 2004, 76(7), 1865-1870. 
39. Faure, K.; Blas, M.; Yassine, O.; Delaunay, N.; Crétier, G.; Albert, M.; Rocca, J.-L. Electrochromatography in Poly(Dimethylsiloxane) Microchips Using Organic Monolithic Stationary Phases. Electrophoresis 2007, 28(11), 1668-1673.

40. Ebara, M.; Hoffman, J. M.; Hoffman, A. S.; Stayton, P. S. Switchable Surface Traps for Injectable Bead-Based Chromatography in PDMS Microfluidic Channels. Lab Chip2006, 6 (7), 843-848.

41. Ma, D.; Chen, H.; Shi, D.; Li, Z.; Wang, J. Preparation and Characterization of Thermo-Responsive PDMS Surfaces Grafted with Poly(N-Isopropylacrylamide) by Benzophenone-Initiated Photopolymerization. J. Colloid Interface Sci. 2009, 332 (1), 85-90.

42. Sugiura, S.; Imano, W.; Takagi, T.; Sakai, K.; Kanamori, T. Thermoresponsive Protein Adsorption of Poly(N-Isopropylacrylamide)Modified Streptavidin on Polydimethylsiloxane Microchannel Surfaces. Biosens. Bioelectron. 2009, 24 (5), 1135-1140.

43. Chen, J. P.; Huffman, A. S. Polymer-Protein Conjugates. II. Affinity Precipitation Separation of Human Immunogammaglobulin by a Poly(N-Isopropylacrylamide)-Protein A Conjugate. Biomaterials 1990, $11(9), 631-634$.

44. Heskins, M.; Guillet, J. E. Solution Properties of Poly(NIsopropylacrylamide). J. Macromol. Sci. Part A - Chem. 1968, 2 (8), 14411455.

45. Cheng, X.; Canavan, H. E.;Stein, M. J.; Hull, J. R.;Kw eskin, S. J.; Wagner, M. S.;Somorjai, G. A.; Castner, D. G.; Ratner, B. D. Surface Chemical and Mechanical Properties of Plasma-Polymerized N-Isopropylacrylamide. Langmuir 2005, 21 (17), 7833-7841.

46. Yakushiji, T.;Sakai, K. Graft Architectural Effects on Thermoresponsive Wettability Changes of Poly (N-Isopropylacrylamide)-Modified Surfaces. Langmuir 1998, 7463 (18), 4657-4662.

47. Du X, Cui H, Sun B, Wang J, Zhao Q, Xia K, Wu T, Humayun MS. Photothermally Triggered Shape-Adaptable 3D Flexible Electronics. Adv. Mater. Technol. 2017, 2(10):1700120.

48. Wang J, Zhao Q, Cui H, Wang Y, Chen H, Du X. Tunable shape memory polymer mold for multiple microarray replications. J. Mater. Chem. A. 2018;6(48):24748-55.

49. Da Silva, R. M. P.; Mano, J. F.; Reis, R. L. Smart Thermoresponsive Coatings and Surfaces for Tissue Engineering: Switching Cell-Material Boundaries. Trends Biotechnol. 2007, 25 (12), 577-583.

50. Patel, N. G.; Zhang, G. Responsive Systems for Cell Sheet Detachment. Organogenesis 2013, 9 (2), 93-100. 
51. Liu, H.; Wang, S. Poly(N-Isopropylacrylamide)-Based ThermoResponsiveSurfaces with Controllable Cell Adhesion. Sci. China Chem. 2014, 57 (4), 552-557.

52. Xia, Y.; Whitesides, G. M. Soft Lithography. Annu. Rev. Mater. Sci. 1998, 28 (1), 153-184.

53. Rohr, T.; Ogletree, D. F.; Svec, F.; Fréchet, J. M. J. Surface Functionalization of Thermoplastic Polymers for the Fabrication of Microfluidic Devices by Photoinitiated Grafting. Adv. Funct. Mater. 2003, $13(4), 264-270$.

54. Yekta-Fard, M.; Ponter, A. B. Factors Affecting the Wettability of Polymer Surfaces. J. Adhes. Sci. Technol. 1992, 6 (2), 253-277.

55. Shui, L.; Van den Berg, A.; Eijkel,J. Interfacial Tension Controlled W/O and O/W 2-Phase Flows in Microchannel. Lab Chip 2009, 9 (6), 795-801.

56. Lee, D.; Weitz, D. A. Double Emulsion-Templated Nanoparticle Colloidosomes with Selective Permeability. Adv. Mater. 2008, 20 (18), 3498-3503.

57. Shum, H. C.; Lee, D.; Yoon, I.; Kodger, T.; Weitz, D. A. Double Emulsion Templated Monodisperse Phospholipid Vesicles. Langmuir 2008, 24 (15), 7651-7653.

58. Herranz-Blanco, B.; Arriaga, L. R.; Mäkilä, E.; Correia, A.; Shrestha, N.; Mirza, S.; Weitz, D. A.; Salonen, J.; Hirvonen, J.; Santos, H. A. Microfluidic Assembly of Multistage Porous Silicon-Lipid Vesicles for Controlled Drug Release. Lab Chip 2014, 14 (6), 1083-1086.

59. Duncanson, W.J.; Lin, T.; Abate, A.R.; Seiffert, S.; Shah, R.K.; Weitz, D. A. MicrofluidicSynthesis of Advanced Microparticles for Encapsulation and Controlled Release. Lab Chip2012, 12 (12), 2135-2145.

60. Chu, L. Y.;Utada, A.S.;Shah, R. K.;Kim,J.W.;Weitz, D. A. Controllable Monodisperse Multiple Emulsions. Angew. Chemie. Int. Ed. 2007, 46 (47), 8970-8974.

61. Shah, R.K.;Shum, H. C.; Row at, A. C.; Lee, D.; Agresti, J. J.;Utada, A. S.; Chu, L. Y.; Kim, J. W.; Fernandez-Nieves, A.; Martinez, C. J.; Weitz DA. Designer Emulsions Using Microfluidics. Mater. Today 2008, 11 (4), 1827.

62. Bai, Z.; Wang, B.; Chen, H.; Wang, M. Spatial Wettability Patterning of Glass Microchips for Water-in-Oil-in-Water (W/O/W) Double Emulsion Preparation. Sensors Actuators B Chem. 2015, 215, 330-336.

63. Abate, A. R.; Weitz, D. A. High-Order Multiple Emulsions Formed in Poly(Dimethylsiloxane) Microfluidics. Small 2009, 5 (18), 2030-2032.

64. Li, S.; Gong, X.; Mc Nally, C. S.; Zeng, M.; Gaule, T.; Anduix-Canto, C; Kulak, A. N.; Baw azer, L. A.; McPherson, M. J.; Meldrum, F. C. Rapid 
Preparation of Highly Reliable PDMS Double Emulsion Microfluidic Devices. RSC Adv. 2016, 6 (31), 25927-25933.

65. Lee, H.; Choi, C. H.; Abbaspourrad, A.; Wesner, C.; Caggioni, M.; Zhu, T.; Weitz, D. A. Encapsulation and Enhanced Retention of Fragrance in Polymer Microcapsules. ACS Appl. Mater. Interfaces 2016, 8 (6), 40074013.

66. Shang, L.; Cheng, Y.; Wang, J.; Yu, Y.; Zhao, Y.; Chen, Y.; Gu, Z. Osmotic Pressure-Triggered Cavitation in Microcapsules. Lab Chip 2016, 16 (2), 251-255.

67. Ye, B.; Ding, H.; Cheng, Y.; Gu, H.; Zhao, Y.; Xie, Z. Photonic Crystal Microcapsules for Label-Free Multiplex Detection. Adv. Mater. 2014, $3270-3274$. 


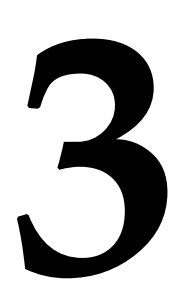

Autonomous Capillary Microfluidic Devices with Constant Flow Rate and Temperature-Controlled Valving 
$\mathrm{A}_{\text {hydrophobic patches as stop valves have been reported, characterized by }}^{\text {utonomous capillary pumping and flow control with switchable }}$ cost-effective fabrication and facile operation. Challenges still remain for full control of single valve-switching and liquid flow for biologically relevant assays. In this chapter, we report on a capillary microfluidic device $w$ ith constant flow rate and temperature triggered stop valve function. It contains a PDMS channel that $\mathrm{w}$ as grafted by a thermo-sensitive polymer Poly (N-isopropylacrylamide) (PNIPAm). The channel exhibits a constant capillary filling speed. By locally increasing the temperature in the channel from $20{ }^{\circ} \mathrm{C}$ to $37{ }^{\circ} \mathrm{C}$ using a microfabricated heater, a change of the surface wettability from hydrophilic to hydrophobic is obtained creating a hydrophobic stop valve. The valve can be reopened by lowering the temperature. The device is simple to fabricate and can be used as an actuatable capillary pump around room temperature. To understand the constant capillary filling speed, we performed contact angle measurements, in which we found slow w etting kinetics of PNIPAm-g-PDMS surfaces at temperatures below the lower critical solution temperature (LCST) of PNIPAm and fast wetting kinetics above the LCST. We interpret this as the result of the diffusive hydration process of PNIPAm below the LCST and the absence of hydration on the hydrophobic PNIPAm thinlayer above the LCST.

tThis chapter is based on the article manuscript: L. Li, E.Y. Westerbeek, J.C. Vollenbroek, S.J.A. de Beer, L. Shui, M. Odijk, and J.C.T. Eijkel. Autonomous capillary microfluidic devices with constant flow rate and temperaturecontrolled valving. Submitted for publication. 


\subsection{INTRODUCTION}

Microfluidic technology allows precise control and manipulation of fluids at the small scale (typically sub- 100 micrometer). ${ }^{1}$ It has been widely used in biological applications such as high-through put screening, ${ }^{2}$ drug delivery, ${ }^{3}$ cellular assays, ${ }^{4}$ as well as point-of-care (POC) diagnostics. ${ }^{5}$ Accurate flow control in microfluidics can be achieved by using either active or passive ways. In active flow-control systems, the flow is controlled using external equipment such as syringe pumps, centrifuges, and electrical or mechanical actuators. ${ }^{6}$ On the other hand, in passive flow-control system, the fluid is pumped utilizing the surface properties and geometric effects at micro-scales such as capillary pumping. For many bioanalytical applications, a passive capillary-driven flow is more attractive due to its spontaneous nature, cost-effective fabrication, and simple operation. $^{7-9}$

Passive capillary-driven flow results from the interfacial energy balance at the meniscus (the solid-liquid-air interface) in microfluidic devices. ${ }^{9}$ Capillary flow can be controlled by valves such as time-delay valves, stop valves, and trigger valves.10-12 Time-delay valves are used to delay the flow of one liquid or to precisely time the delivery of multiple different liquids. Commonly used approaches to create time-delay valves are manufacturing a widened section in the flow channel or locally increasing the surface hydrophobicity. Another approach to creating time delays is by implementing a dissolvable barrier, e.g a dissolvable thin film. ${ }^{13}$ Stop valves can be based on the same principles as delay valves. One approach is to abruptly enlarge the channel diameter, ${ }^{14,15}$ and another locally reversing the surface wettability from hydrophilic to hydrophobic. Different approaches have been reported, such as hydrophobic patterning ${ }^{11}$ using stimulus materials to create reversible surface wettability, ${ }^{16,17}$ or using a hydrogel with changeable volume. ${ }^{18-20}$ Trigger valves are basedon two liquid fronts merging to enable further movement of the liquid in a common outlet. This type of trigger valve has been widely used for controlling of sequential liquid delivery for high-throughput screening..$^{21}$ The autonomous driving process of capillary flow and the different valve functionalities make capillarity-based microfluidic devices highly useful tools for clinical and bioanalytical tests. Apart from the use of valves, control of the flow rate in capillary devices is difficult exactly due to the autonomous nature. In capillary channels of constant cross-section, the liquid behavior is generally believed to follow the well-known Washburn behavior, characterized by a flow rate that 
decreases with $\sqrt{t} .{ }^{22}$ The seminal work of Delamarche ${ }^{23}$ shows metering and operation in autonomous capillary devices at constant flow rate by the geometric device design. Such a solution can improve diagnosticassay performance.

Poly (N-isopropylacrylamide) (PNIPAm) is a thermosensitive polymer that has a low er critical solution temperature (LCST) of $32^{\circ} \mathrm{C}$. It has been widely used for surface treatment of microfluidic devices to control autonomous liquid flow due to its thermo-responsive hydrophilic/hydrophobic properties. One group has previously reported on a PNIPAm valve in a microfluidic device, ${ }^{16,17,24}$ created by local grafting of the channel surface and then heating the entire device. This choice has the drawback that in a device with multiple valves all valves switch simultaneously, making single-valve control impossible. The authors furthermore operated the valve by applying a wide temperature range (from room temperature to $55^{\circ} \mathrm{C}$ ).

In this chapter, we introduce a PNIPAm grafted polydimethylsiloxane (PNIPAm-g-PDMS) capillary microfluidic device with constant flow rates and local temperature-controlled valving. By locally varying the temperature of the channel surface between 20 and $36^{\circ} \mathrm{C}$, the surface can locally be switched from hydrophilic to hydrophobic thus obtaining a local valving function. This temperature range makes the valve suitable for most biomedical applications. The device we present here provides larger oper ational freedom, enables singlevalve control, and operates at a more convenient temperature range.

In addition, we found that the device is characterized by a capillary filling rate that is constant in time. This finding is of great interest for liquid control in microfluidic devices. In this chapter, we explain the constant flow rate by a dynamic contact angle, caused by the diffusive hydration of the PNIPAm film. Moreover, we demonstrate that the observed oscillating velocity is attributed to the slip-jump of the contact line due to differently w ettable opposing channel walls.

In short, in this chapter, we offer a solution for full control of single valveswitching in capillary devices and present a further study of the liquid filling behavior in PNIPAm coated microfluidic devices. 


\subsection{WORKING PRINCIPLE}

(a)

(i) microfluidic device

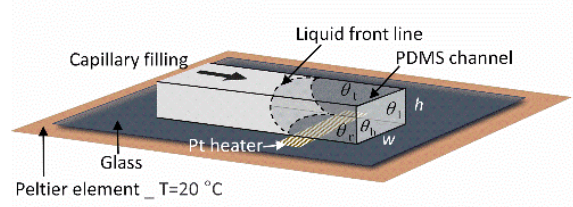

(ii) cross-section view

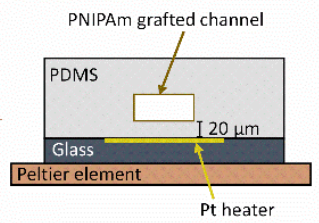

(b)

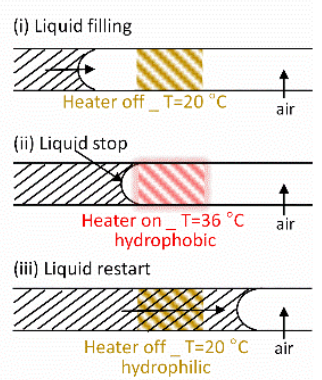

Figure 3.1 Schematic drawing of liquid filling and temperature-controlled valving in a straight rectangular channel of constant width $w$ and height $h$. (a) Capillary filling in a rectangular channel with differently wettable walls. $\theta_{b}, \theta_{t}, \theta_{l}, \theta_{r}$ are the contact angles of the liquid on the bottom, top, left, right walls, respectively. A heater was fabricated underneath the channel to locally control the temperature. A Peltier element controlled at a constant temperature of $20^{\circ} \mathrm{C}$ was placed under the chip to confine the heated area. (b) Autonomous liquid filling (i) at $20^{\circ} \mathrm{C}$; liquid stop (ii) by locally heating at $36{ }^{\circ} \mathrm{C}$ making the surface hydrophobic; liquid restart (iii) by turning off the heater and allowing the temperature to return to $20^{\circ} \mathrm{C}$.

Figure 3.1a shows a schematic drawing of the microfluidic chip (i) and the cross-section of the channel (ii). The channel is made from PDMS and coated with the thermo-responsive polymer PNIPAm. As a result, the channel surface shows a variable wettability, being hydrophilic at temperatures below its LCST $\left(32^{\circ} \mathrm{C}\right)$, and becoming hydrophobic at temperatures above $36^{\circ} \mathrm{C}$. Figure $1 \mathrm{~b}$ shows the valving process. At room temperature, aqueous solution fills the hydrophilic channel by the capillary driving force at the liquid front (Figure 3.1b(i)). When the channel is locally heated up to $36^{\circ} \mathrm{C}$, the PNIPAm-g-PDMS surface forms a hydrophobic patch which will stop the flow (Figure 3.1b(ii)). When we turn off the heater, the temperature drops to $20^{\circ} \mathrm{C}$ and liquid filling spontaneously restarts since the surface becomes hydrophilic again (Figure $\mathbf{3 . 1 b}$ (iii)).

Precise control of flow rate is of great importance in many applications. Here we will investigate both the filling and valving function, based on the surface properties of our device. 


\subsubsection{Laplace pressure and valving}

For microchannels of a rectangular cross-section, the capillary pressure $P$ of a liquid/air meniscus is ${ }^{1}$

$$
P=-\gamma_{l v}\left(\frac{\cos \theta_{b}+\cos \theta_{t}}{h}+\frac{\cos \theta_{l}+\cos \theta_{r}}{w}\right)
$$

Here $\theta_{b}, \theta_{t}, \theta_{l}, \theta_{r}$ are the contact angles of the liquid on the bottom, top, left, right $\mathrm{w}$ alls, respectively, and $h$ and $w(\mathrm{~m})$ are the height and width of the microchannel. A schematicdrawing of channel geometry is shown in Figure 3.1a. Each channel wall with a hydrophilic surface $\left(\theta<90^{\circ}\right)$ contributes to generating a negative pressurein front of the liquid meniscus, drawing the liquid into the channel. For a channel with differently wettable walls, the filling process depends on the joint action of the four channel walls and the cross-sectional geometry. When equation (1) results in a positive Laplace pressure, the liquid movement willstop.

In our experiments, the four channel walls are coated with PNIPAm, making the w ettability of the channel surfaces thermo-sensitive. The capillary pressure $P$ will thus depend on the temperature distribution over the channel surfaces. By locally controlling the temperature, we can obtain liquid filling, stopping, and restarting in the channel(Figure $3.1 \mathrm{~b}$ ).

\subsubsection{Filling behavior}

\subsubsection{Constant filling speed}

The capillary filling process of a rectangular microfluidic channel with width $w>>$ height $h$ is generally described by the Lucas-Washburn equation ${ }^{22,25}$, balancing the driving force resulting from the surface tension and the resistive viscous force:

$$
2 w \gamma l w \cos \theta_{s}=\frac{12 \pi w}{h} l(t) \frac{d l(t)}{d t}
$$

Here $\gamma_{l v}$ is the surface tension of the liquid $(\mathrm{N} / \mathrm{m}), \theta_{s}$ is the static liquid/solid contact angle in the channel, $l(t)(\mathrm{m})$ is the length of the filled channel section at time $t(\mathrm{~s})$ and $\eta\left(\mathrm{N} \cdot \mathrm{s} \cdot \mathrm{m}^{-2}\right)$ is the dynamic viscosity of the liquid.

In a number of papers, it has been shown that the dynamic contact angle depends on the filling speed, which is attributed to hydrodynamic and wall surface forces. ${ }^{26-30}$ In the case of the PNIPAm film/water system, a different process causes a dynamic contact angle. The contact angle measurements shown in Figure 3.4 indicate that the contact angle of a macroscopic water drop on a dry 
PNIPAm film changes in about 30 seconds from about $100^{\circ}$ to about $60^{\circ}$. We contribute this slow hy drophilization to the diffusive hydration of the PNIPAm film. In a microchannel, the moving meniscus will thus be preceded by a region in which the PNIPAm brush is progressively hydrated (Figure 3.2). In the case that the PNIPAm hy dration solely determines the meniscus velocity $d l(t) / d t$, and the viscous dissipation along the liquid column can be neglected, it can be expected that the contact angle at the meniscus is just slighty below $90^{\circ}$. We define $d$ as the axial extension of the diffusion region that under these conditions precedes the meniscus. Over this distance $d$, the contact angle decreases from the non-hydrated value of $\sim 110^{\circ}$ to $90^{\circ}$. From the location of the moving meniscus forw ard, this region will move with an axial velocity of $\alpha D / d$ with $D$ the diffusion coefficient of $w$ ater in PNIPAm and $\alpha$ a numerical constant with a value between 2 (1D diffusion) and 4 (2D diffusion). At locations behind the liquid meniscus, the diffusive hy dration will further proceed and the contact angle $w$ ill decrease until $\theta_{s}$ is reached. With increasing length of the filled channel, the viscous dissipation along the liquid column increases, decreasing the meniscus velocity $d l(t) / d t$, and decreasing the dynamic contact angle until the static contact angle is reached. We can thus approximate the dynamic contact angle by an equation combining the diffusional hydration velocity and the meniscus velocity,

$$
\cos \theta_{d}=\cos \theta_{s}\left(1-\frac{d}{\alpha D} \cdot \frac{d l(t)}{d t}\right)
$$

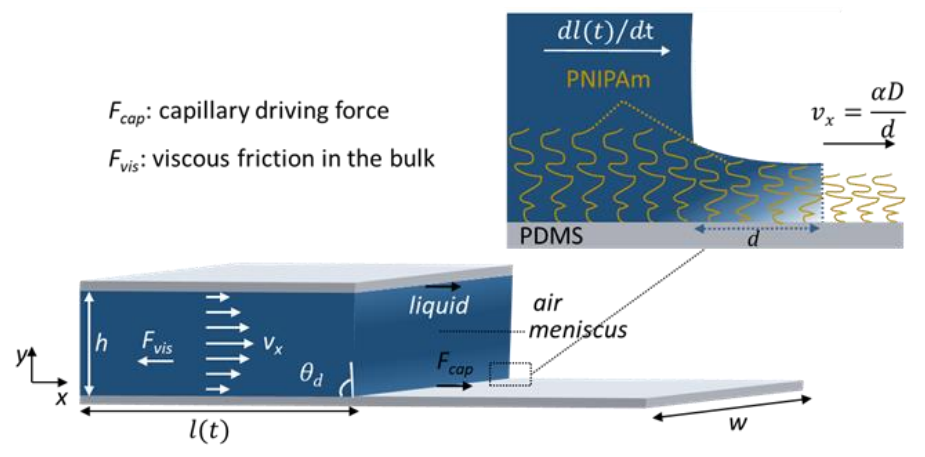

Figure 3.2. Schematic drawing of the filling process. The liquid fills in the channel with a height of $h$ and width of $w$. At the meniscus the dynamic contact angle is $\theta d$, resulting in a capillary driving force $\left(F_{c a p}\right)$ balanced with the viscous resistance force $\left(F_{v i s}\right)$ in the liquid bulk. When the viscous resistance force can be neglected, the contactangle at the meniscus equals $\sim 90^{\circ}$ and the meniscus velocity $d l(t) / d t$ is equal to the diffusional penetration velocity of water in the PNIPAm film, $v_{x}=\alpha D / d$, with $D$ the diffusion coefficient of wate $\mathrm{r}$ in PNIPAm and $d$ the axial extension of the diffusion layer in front of the meniscus. 
From equations 2 and 3 we obtain

$$
\gamma_{l v} \cos \theta_{s}\left(1-\frac{d}{\alpha D} \cdot \frac{d l(t)}{d t}\right)=\frac{6 \eta}{h} l(t) \frac{d l(t)}{d t}
$$

When $d l(t) / d t \ll \alpha D / d$, we retrieve the Lucas-Washburn equation, with $l$ proportional to the square root of time,

$$
l(t)=\sqrt{\frac{h \gamma_{h} \cos \theta_{s}}{3 \eta} t}
$$

When $d l(t) / d t \approx \alpha D / d$, filled length $l$ will be proportional to time,

$$
l(t)=\frac{\alpha D}{d} t
$$

The experimentally determined meniscus velocity in case of a constant filling rate is of the order of $100 \mu \mathrm{m} / \mathrm{s}$, and the water diffusion coefficient in a PNIPAmbrush $D=1.5 \cdot 10^{-11} \mathrm{~m}^{2} / \mathrm{s}^{, 31}$ resulting in $d=150 \alpha \mathrm{nm}$. Assuming that $2<\alpha<4$ (between 1D and 2D diffusion), we find that $d$ is in the order of the thickness of the PNIPAm brush.

We observed frequent contact line pinning as well as anirregular contact line in our system (see Video 1, 2, 3 in Appendix B). Both the height variations of the PNIPAm brush (Figure 3.4b) as the deformation of the PNIPAm at the contact line on water intrusion can play a role here. Pinning and depinning in a PNIPAM-clad channel was also described by Silva et al., though these authors used a capillary with a $10 \mu \mathrm{m}$ dry thickness PNIPAm gel layer, where pinning due to mechanical deformation of the PNIPAm gel is expected to play a much larger role than in our system where the PNIPAm brush has a submicrometer thickness. 32

3.2.2.2 Different w ettability of the opposing channel walls, causing an oscillating contact line velocity

Interestingly, we observed velocity oscillations on the $100 \mathrm{~ms}$ time scale. We explain this from a different wettability of the top and bottom channel walls in our system, which results from the manufacturing process. The water penetration into the PNIPAm will then proceed with a different velocity at the top and bottom walls of the channel. A peculiar meniscus profile $w$ ill be formed, with the fastest moving contact line leading and the slowest moving contact line following. In the absence of pinning, this meniscus will move with a constant velocity intermediate to the two contact lines. When pinning of the slowest 
contact line occurs, the contact angle at the slowest wetting surface will further increase by the movement of the fastest contact line until it leads to depinning of the pinned slow contact line by the change of contact angle. The depinning will be follow ed by a rapid advance of the slow contact line due to the energy stored in the meniscus. A sequence of subsequent pinning and depinning $w$ ill result in an oscillating velocity of both contact lines. Assuming that pinning events frequently occur and depinning occurs when the fastest meniscus has advanced over a length of the order of the channel height $h$, it is expected that the temporal frequency $f$ at which these slip-jumpevents occur is proportional to the meniscus velocity and inversely proportional tochannel height $h\left(f \alpha \frac{\left(\frac{\alpha D}{d}\right)}{h}\right), \mathrm{w}$ ith $\Delta\left(\frac{\alpha D}{d}\right)=\left(\frac{\alpha D}{d}\right)$ $\left(\frac{\alpha D}{d}\right)_{b}$.

3.2.3 Temperature distribution along the channel walls at liquid/air interface

For the temperature distribution at the channel walls at the water-air interface, a three-dimensional, time-dependent heat equation $w$ as solved for the geometry as indicated in Figure 3.3a using COMSOL 5.5,

$$
\rho C_{p} \frac{\partial T}{\partial t}+\nabla \cdot(-k \nabla T)=0
$$

Here $\rho$ is the density $\left(\mathrm{kg} \cdot \mathrm{m}^{-3}\right), C_{p}$ the heat capacity $\left(\mathrm{J} \cdot \mathrm{kg}^{-1} \cdot \mathrm{K}^{-1}\right), T$ the temperature $(\mathrm{K}), t$ the time $(\mathrm{s})$, and $k$ the thermal conductivity $\left(\mathrm{W} \cdot \mathrm{m}^{-1} \cdot \mathrm{K}^{-1}\right)$. Since $P e<<1$, heat transport due to convection was neglected. The air-liquid interface was considered to be static. The air-liquid interface was considered to be static. The boundary conditions are displayed in Figure 3a, the heater was simulated as a 1D line with a constant temperature. The material properties used in the simulation can be found in Appendix B.6.

Because of the symmetry of both heater and channel, only half the channel was simulated. The simulation results are shown in Figure 3.3, using the boundary conditions mentioned in the caption, indicating that for a channel of $100 \mu \mathrm{m}$ width and $35 \mu \mathrm{m}$ height, both the temperature along the channel sidewall (Figure 3.3b) and the top channel wall (Figure 3.3c) reaches values above $36^{\circ} \mathrm{C}$ within $1 \mathrm{~s}$ of switching the platinum heater filament temperature from $20^{\circ} \mathrm{C}$ to $40^{\circ} \mathrm{C}$. We deem this a sufficiently fast temperature response. 


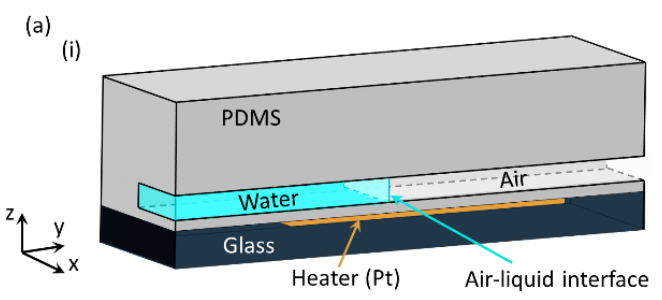

(b) sidewall cross-section

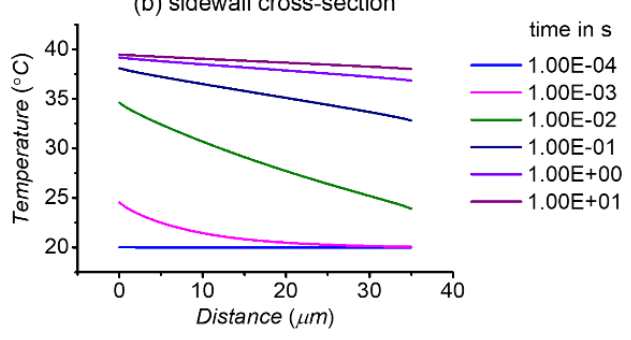

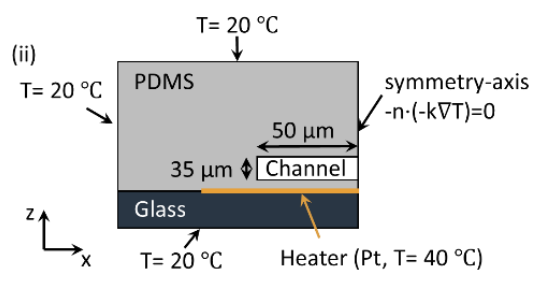

(c) topwall cross-section

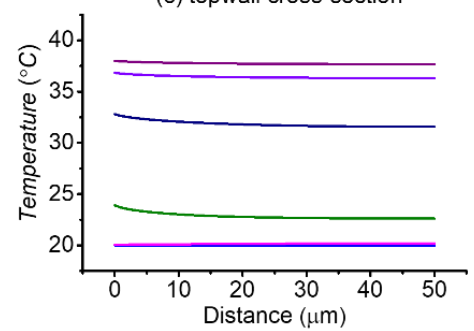

Figure 3.3. Simulation results of the temperature distribution along channel walls. (a) Schematic drawing of the microfluidic chip (i) and the cross-sectional view (ii) (not on scale) showing materials and boundary conditions; $\mathrm{T}=20^{\circ} \mathrm{C}$ for the outer boundaries of the chip and the inlets and $\mathrm{T}=40{ }^{\circ} \mathrm{C}$ for the Platinum. At the symmetry-axis $-\mathbf{n} \cdot(-k \nabla T)=0$. The standard solver settings were used. The liquid-air interface was modeled at the middle of the platinum. A finer mesh was used at the liquid-air interface, and a coarser mesh was used in the rest of the model. The specific mesh details and geometry of the simulation can be found in the COMSOL file, which is added in the Appendix B.6. (b) The temperature distribution at the liquid-air interface along the channel side wall ( $x=0$ to 35 $\mu \mathrm{m})$. (c) The temperature distribution at the liquid-air interface along the to $p$ channel wall ( $x=0$ to $50 \mu \mathrm{m}$ ), taken at times $10^{-4}, 10^{-3}, 10^{-2}, 10^{-1}, 1$ and $10 \mathrm{~s}$.

\subsection{MATERIALS AND METHODS}

\subsubsection{Materials}

N-Isopropylacrylamide (NIPAm, CAS: 2210-25-5), benzophenone (CAS: 119-619), $\mathrm{NaIO}_{4}$ (CAS: 7790-28-5), and benzyl alcohol (CAS: 100-51-6) were all obtained from Sigma-Aldrich (the Netherlands). Negative photoresist SU-8 3050 and developer solution were purchased from MicroChem (MA, USA) for fabricating the silica mold with the designed microchannels on a Si wafer (525 $\mu$ m thick, Okmetic, Finland). Positive photoresist OiR907-17i (Fujifilm, Japan) w as used for fabricating the platinum patterns. The poly (dimethylsiloxane) (PDMS, Sylgard 184) package was purchased from Dow Corning Corporation (MidlandMichigan, USA) and w as used for fabricating the microfluidic chip. 


\subsubsection{Heater and sensor fabrication}

A patterned platinum heater and sensor structure $w$ as manufactured on glass by standard photolithography, a wet-etching (recess) process, and sputtering. A positive resist(PR) layer (OiR907-17i, Fujifilm, Japan) of 1.71 $\pm 0.04 \mu \mathrm{m}$ thickness was spin-coated on a glass wafer (525 $\mu \mathrm{m}$ thick, Okmetic, Finland) at $4000 \mathrm{rpm}$ for $30 \mathrm{~s}$, followed by baking on a hot-plate at $95^{\circ} \mathrm{C}$ for $2 \mathrm{~min}$. A layer of a few nanometer-thick hexamethyldisilazane (HMDS) was spin-coated on the glass w afer before spin-coating the PR layer. This HMDS layer is used to increase the adhesion of patterned PR structures with the substrates. The exposure process w as conducted by using a mask alignment sy stem (EVG620, EV Group, Austria) for $3 \mathrm{~s}$ at an intensity of $12 \mathrm{~mW} \cdot \mathrm{cm}^{-2}$ in hard contact mode. Thereafter, the wafer w as post-baked on a hot plate at $120^{\circ} \mathrm{C}$ for $15 \mathrm{~s}$, followed by developing in an OPD4246 developer for $1 \mathrm{~min}$, and rinsing with deionized (DI) w ater to complete the fab rication of the pattern for the platinum structures on a glass substrate. BHF $\mathrm{w}$ as then been used as a w et etchant to etch the heater pattern $200 \mathrm{~nm}$ deep into the glass substrate. This process w as directly followed by the sputtering of a 20 $\mathrm{nm}$ thick tantalum (Ta) adhesion layer and a $180 \mathrm{~nm}$ thick platinum $(\mathrm{Pt})$ layer, causing the heater to be embedded into the glass substrate. Lastly, PR layer is lift-off hereby also removing excess TA/Pt on top of the PR layer.

\subsubsection{Temperature sensor calibration}

The temperature sensor calibration w as done by mounting the chip on a Printed Circuit Board (PCB) and wire bonding the electrodes to the PCB, so the chip can be addressed via external connectors. The chip w as fully immersed in a beaker of olive oil (AH Olijfolie mild) standing on a hotplate, type IKA RET. A commercially available thermocouple (Fluke 51 II, Fluke Corporation, United States), w as used as a reference thermometer and measured the temperature of the oil. A magnetic stirring bead was added to increase the uniformity of the temperature in the oil. The hotplate w as heated from $20^{\circ} \mathrm{C}$ to $100{ }^{\circ} \mathrm{C}$ while the resistance of the temperature sensor $\mathrm{w}$ as measured. The change in resistance to the temperature measured by the sensors is shown in Figure B1 (see Appendix B).

\subsubsection{PDMS device fabrication}

Designed microchannel patterns w ere transferred on a SU-8layer spin-coated on a silicon wafer, to serve as master mold using standard phot olithography techniques. ${ }^{33}$ PDMS pre-polymer and curing agent were mixed using a stirring 
machine at a mass ratio of 10:1 and then degassed in a vacuum chamber. The mixture was then cast ontothe master mold to form the channel layer. Also, a 20 $\mu \mathrm{m}$ thick PDMS layer was made on a glass slide by spin coating a PDMS mixture at $3000 \mathrm{rpm}$ for $70 \mathrm{~s}$. Then the PDMS on the master mold and the glass slide with a thin PDMS layer was thermally cured in an oven at $60^{\circ} \mathrm{C}$ for $50 \mathrm{~min}$. The PDMS replica with designed channel patterns was then peeled from the silicon master and cut into the predesigned size and bonded with the glass slide with PDMS layer by thermal bonding in an oven at $60^{\circ} \mathrm{C}$ for 15 hours. Before bonding, a liquid reservoir at both the inlet and outlet of the channel was created by punching holes in the PDMS using a puncher with a $3 \mathrm{~mm}$ inner diameter.

3.3.5 UV-induced surface grafting of NIPAm on PDMS mediated by benzophenone

UV-induced grafting of PNIPAm was conducted by UV-initiated surface polymerization, ${ }^{34,35}$ a schematic drawing of the grafting process is shown in Figure B2 (see Appendix B). Benzophenone-acetone (20 wt $\%$ ) solution was introduced into the PDMS channels for $5 \mathrm{~min}$, followed by a deep wash of the channels with DI water and ethanol. Then the monomer solution containing NIPAm $(10 \mathrm{wt} \%), \mathrm{NaIO}_{4}(0.5 \mathrm{mM})$, and benzyl alcohol (0.5 wt $\left.\%\right)$ was introduced into the channel through tubing connected to the inlet using manual syringe pumping. The device $w$ as subsequently placed in an oven with UV irradiation ( $210 \mathrm{~W}, 365 \mathrm{~nm}$, IntelliRay 600, Uvitron International Inc., USA) to graft the PNIPAm ontothe PDMS surfaces, and then the channel was washed extensively with DI w ater to remove residual monomer and polymer. An ice-water bath was then used to keep the temperature of the monomer solution below the LCST of NIPAm.

\subsubsection{Contact angle measurements}

A single water drop of small volume $(2-12 \mu \mathrm{L})$ was dripped gently on the PNIPAm-g-PDMS substrates. An interfacial tension meter OCA 15 Pro (Dataphysics, Germany) was used to measure the static contact angle or dynamic contact angle. The w ater wetting process on PNIPAm-g-PDMS below the LCST $\left(22{ }^{\circ} \mathrm{C}\right)$ and above the LCST $\left(37^{\circ} \mathrm{C}\right)$ including the advancing, wetting, and receding states were investigated by increasing/decreasing the volume of the w ater droplet. Each substrate $w$ as measured for five times at different positions to obtain the average value of w etting contact angles. 


\subsubsection{Capillary filling measurement procedure and data processing}

The glass chip with platinum heaters and sensors was placed into a Polyoxymethylene chip holder. A Peltier element $w$ as placed underneath the chip to ensure steep temperature gradients around the heaters to prevent elevating the temperature of the entire glass chip. For control of the heaters, a Labview PI controller was w ritten and executed on the myRIO-1900 (National Instruments, U.S.A). Details of the controller were reported in previous w ork of our group..$^{36}$ The temperature sensors fabricated in the glass chip thereby provide the feedback temperature for a programmed control loop. In all experiments, the temperature of the Peltier element was set at a constant temperature of $20^{\circ} \mathrm{C}$. Before the filling process, PDMS chips were grafted with PNIPAm. After drying by air blow, the chip w as then used to study the filling process. For this purpose, $6 \mu \mathrm{L}$ of DI water was pipetted in the reservoir. The filling behavior of the channels w as observed using a Leica DM $6000 \mathrm{Mmicroscope}$ with a Leica DFC 420 digital camera (Wetzlar, Germany). Captured pictures and videos were analyzed using MATLAB R2019a and ImageJ (version: 2.0.0-rc-69/1.52p) to calculate the location and velocity of liquid contact lines in the channel. For the data processing, we first used ImageJ to convert each frame to grayscale and determined the frame rate, frame number, and pixel size. Then we used MATLAB to track the liquid meniscus movement by comparing differences between frames. The absolute difference between successive frames $w$ as used to divide an image frame into changed and unchanged regions. Since only the liquid meniscus moves, we expect the changed region to be associated only within the channel area. We thus obtained the liquid meniscus movement in pixel numbers. Subsequently, location, time, and velocity were calculated from the pixel number and known frame rate, and pixel size. The motion of the contact line w as then filtered by a Gaussian filter. Then meniscus location (filled length) and liquid velocity were plotted against time. To show the periodical oscillation of the contact line, firstly, the middle part of the channel was cropped from the movie by Imagej; secondly, the contrast of the frames was adjusted to the maximum value; finally, we calculated the percentage of the black area of the total area of the entire frame as changing in time $w$ as calculated. 


\subsection{RESULTS AND DISCUSSION}

\subsubsection{Surface characterization of PNIPAm-g-PDMS}

Before surface treatment, bare PDMS shows a poor wettability for water with a $\mathrm{CA}$ of about $108^{\circ}$. This hydrophobicity is ascribed to the repeating - $\mathrm{OSi}\left(\mathrm{CH}_{3}\right)_{2-}$ units on the surface. UV ir radiation in a NIPAm-containing solution induces the generation of free radicals which cause NIPAm monomers to polymerize on the treated PDMS surface. At temperatures below its LCST, molecular water in the atmosphere interacts with the PNIPAm chains in the PNIPAm thin film driven by enthalpy gain, ${ }^{37}$ and the PNIPAm shows hydrophilic properties $w$ ith a static water CA of $67^{\circ}$ at $22{ }^{\circ} \mathrm{C}$. At temperatures above the LCST, PNIPAm chains collapse resulting in the absence of hydrogen bonds between amide groups and water molecules. Atmospheric water is rejected by the surface layer of dehydrated PNIPAm film, resulting in a hydrophobic surface ${ }^{38} \mathrm{w}$ ith a static CA of $93^{\circ}$ at $36^{\circ} \mathrm{C}$. Schematic drawings of PNIPAm chain hydration at temperatures below and above the LCST and the corresponding static CA are shown in Figure 3.4a. Figure 3.4b shows the surface roughness of the PNIPAm-g-PDMS surface (Atomic force microscopy (AFM) microscopy, tapping mode, Cypher ES Environmental AFM, Oxford Instruments, UK). A roughness parameter (Ra) of $3.57 \mathrm{~nm}$ was obtained on a surface prepared with a UV-irradiation time of $10 \mathrm{~min}$. The thickness of the PNIPAm layer, in this case, is around $430 \mathrm{~nm}$. Figure 3.4c show s the FT-IR spectrum (FT-IR Spectrometer INVENIO®, Bruker, Germany) using a sample cell equipped with a ZnSe single crystal in ATR mode. The PNIPAm-g-PDMS surface shows new absorbance peaks at $\sim 3300 \mathrm{~cm}^{-1} \sim 1650$ $\mathrm{cm}^{-1}, \sim 1550 \mathrm{~cm}^{-1}$ compared to the spectrum of the bare PDMS surface. These new peaks can be assigned to the $\mathrm{N}-\mathrm{H}$ stretching vibration, amide $\mathrm{I}(\mathrm{C}=\mathrm{O}$ stretching vibration), and amide II bonds (N-Hbending). All features indicate that PNIPAm has been grafted on the PDMS surface. 


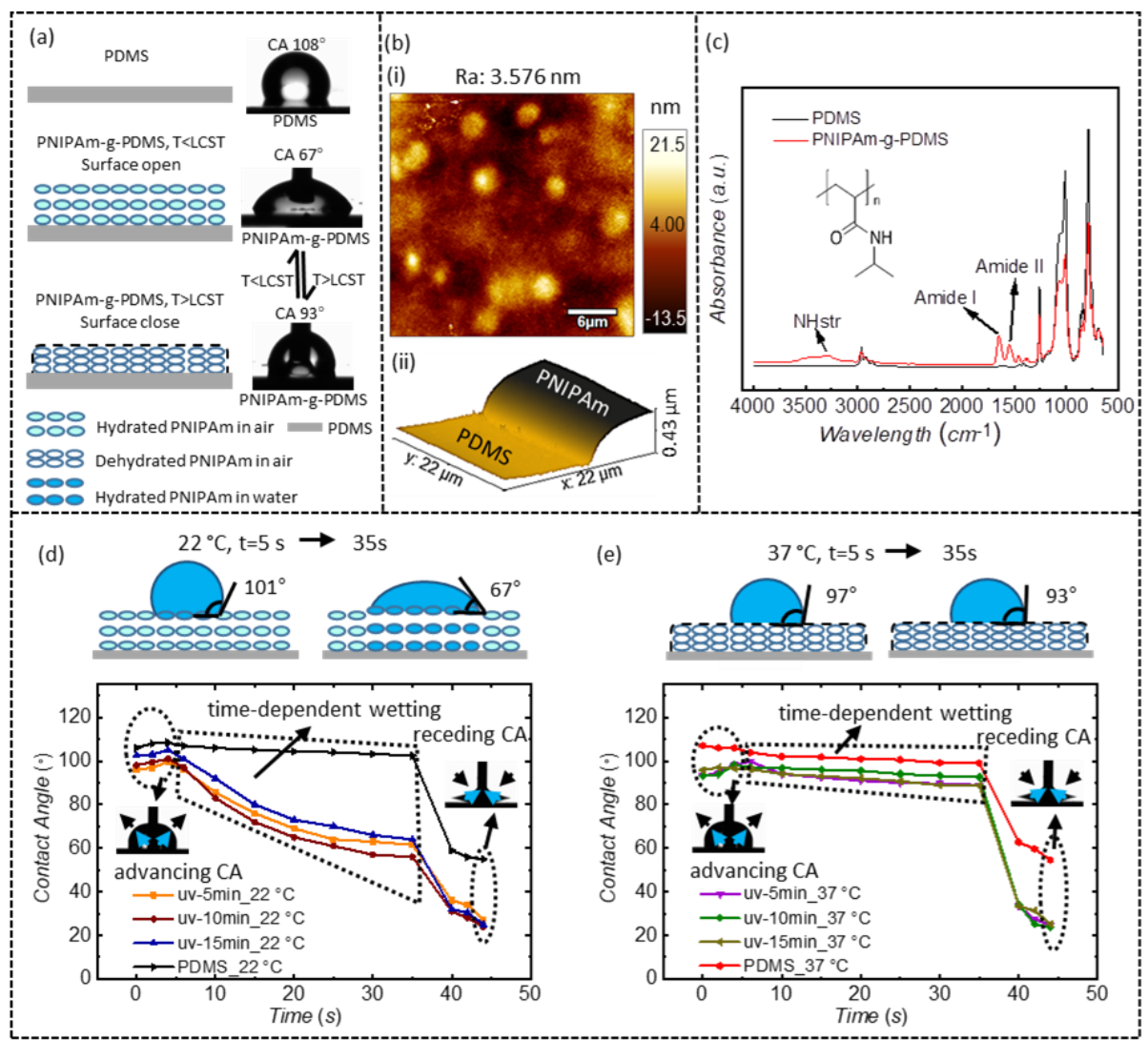

Figure 3.4. Wetting properties of a PNIPAm-g-PDMS surface. (a) The static water CA on PDMS, PNIPAm grafted on a PDMS surface. At $22^{\circ} \mathrm{C}(\mathrm{T}<\mathrm{LCST})$, water molecules can form hydrogen bonds with the polymer chain, results in ahydrophilic surface with a static CA of $67^{\circ}$. At $36{ }^{\circ} \mathrm{C}(\mathrm{T}>\mathrm{LCST})$, water molecules in the air are rejected by the shrink PNIPAm surface, results in a hydrophobic surface with a static CA of $93^{\circ}$. (b) FT-IR spectra of the PDMS and PNIPAm-g-PDMS. (c) Wetting behavior of PNIPAm-g-PDMS surfaces at $20^{\circ} \mathrm{C}$, showing the advancing CA (before 5 seconds) and receding CA (after 35 seconds) and time-dependent wetting behavior to reach static CA on PDMS and PNIPAm-g-PDMS surfaces prepared under UV irradiation for times of 5, 10, $15 \mathrm{~min}$. The schematic drawing above shows the contact line moving from highly hydrated PNIPAm to less hydrated PNIPAm dry film in the air during the time-dependent wetting process. (d) wetting properties of PDMS and PNIPAm-g-PDMS surfaces at $40{ }^{\circ} \mathrm{C}$, which show a similar advancing and receding angle but no obvious time-dependent wetting behavior which is due to the dehydrated PNIPAm on the surface. The schematic drawing above shows the absence of obvious contact line movement after deposition of the water drop.

The measured wetting behavior of water on PNIPAm-g-PDMS is shown in Figure $3.4 \mathrm{~d}$ and e. At $22^{\circ} \mathrm{C}$, the PNIPAm-g-PDMS surface shows an advancing 
CA of $\sim 96^{\circ}$ and a receding CA of $\sim 33^{\circ}$. This contact angle hysteresis indicates contact line pinning on the surface. Furthermore, at $22^{\circ} \mathrm{C}$ the surface shows timedependent wetting, which we ascribe to the slow swelling and hydration of the PNIPAm chains in water as described above. At $37^{\circ} \mathrm{C}$ how ever, the surface wetting is practically time-independent, which we ascribe to the absence of a hydration process of the PNIPAm film. The non-hydrated PNIPAm at temperatures above the LCST causes a hydrophobic PNIPAm-g-PDMS surface. At $37^{\circ} \mathrm{C}$, the surface still shows contact angle hysteresis with the advancing CA of $\sim 96^{\circ}$ and the receding CA of $\sim 33^{\circ}$, resulting from contact line pinning. Pelton ${ }^{39}$ described how the hydrophobicity of PNIPAm, as judged by the contact angle, is a strong function of the properties of the contact phase.

3.4.2 Wetting properties of different channel walls and corresponding Laplace pressure

Since the thickness of the PDMS layer at the channel bottom $(20 \mu \mathrm{m})$ in our experiments is much smaller than that at the top and side $w$ alls ( $\mathrm{mm}$ scale), the concentration of the photoinitiator benzophenone diffused in the PDMS before the grafting of the NIPAm will be different at these different locations. Since the photoinitiator generates radicals that accelerate the grafting rate of monomerson the PDMS surface, the concentration of benzophenone is a key factor in the grafting process. ${ }^{40}$ To estimate the benzophenone concentration in the different channel w alls, we used the fluorescent Rhodamine 6G at an equal concentration as the photoinitiator in acetone as a diffusion indicator. ${ }^{41}$ The results are shown in Figure B3 (in Appendix B), indicating that theconcentration of benzophenone in the bottom PDMS layer is much higher than in the top wall and sidewalls. We, therefore, assume that the wetting properties of the top and side walls are identical, and $\theta_{t}, \theta_{l}$, and $\theta_{r}$ in equation (1) are equal, but that $\theta_{t}$ differs. We therefore only need to measure the CA on the top and bottom wall of the channel.

The CA measurement procedure is described in 3.3.6 and results are shown in Figure 3.5. Static contact angles on PNIPAm-g-PDMS surfaces prepared under 5, $10,15 \mathrm{~min}$ of UV exposure were investigated after different storage times. 
(a)
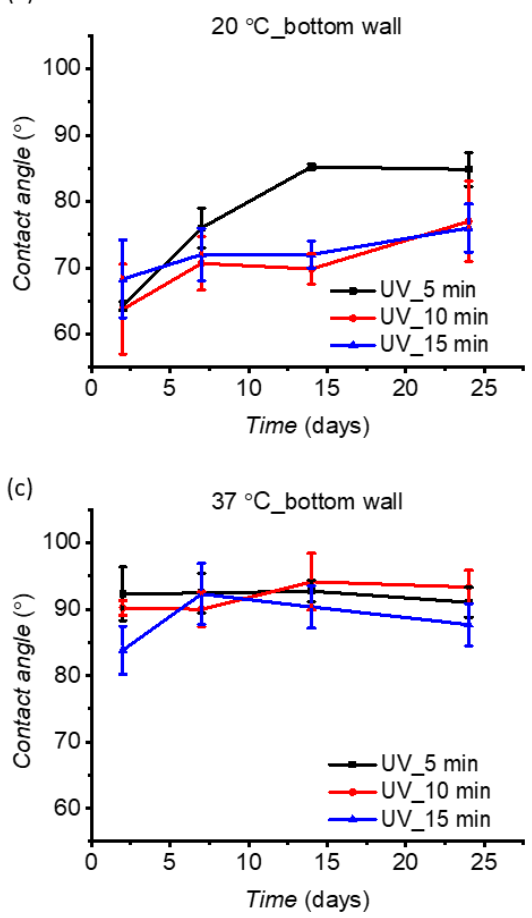

(b)

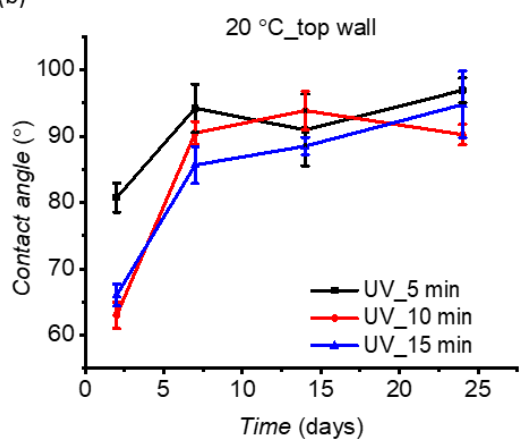

(d)

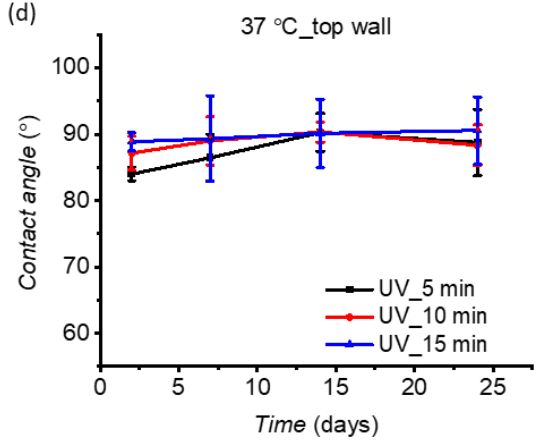

Figure 3.5. Static contact angle on the channel walls changes with time at 20 and $37^{\circ} \mathrm{C}$. The error bars represent the standard deviation calculated from 5 measurements from different locations on the surface for each data point.

From Figure 3.5a, we see that the CA on the bottom wall (thin PDMS layer) increases quickly in the first week of stor age and then more slowly after around 2 w eeks. It remains below $90^{\circ}$ even after 23 days. The increase of the CA during storage can be either ascribed to a slow process of movement of hydrophobic groups to the polymer surface or of PDMS oligomers from the bulk to the surface, which processes can be easily influenced by environmental factors like humidity and temperature. ${ }^{42}$ The CA change can also be caused by contamination from the atmosphere. The top w all after two days is less hydrophilicthan the b ottom wall and with further storage, the CA of the bottom wall increases to $90^{\circ}$ or higher (Figure 3.5b). As mentioned above, we ascribe this to the lower concentration of benzophenone in the PDMS of the top and side $\mathrm{w}$ alls which results in a thinner PNIPAm layer. ${ }^{43}$ Figure 3.5 $\mathrm{c}$ and $\mathrm{d}$ show the w ater CA on the bottom $w$ all and top w all at $37^{\circ} \mathrm{C}$. As this tem perature is above its LCST, the PNIPAm molecules contract to a compact conformation and form small hydrophobic nano-pockets. ${ }^{4}$ 
Such a compact state makes the surfaceless hydrophilicor even hydrophobic. In our experiment, the w ater CA on a PNIPAm-g-PDMS surface at $37^{\circ} \mathrm{C}$ increased to $90^{\circ}$.

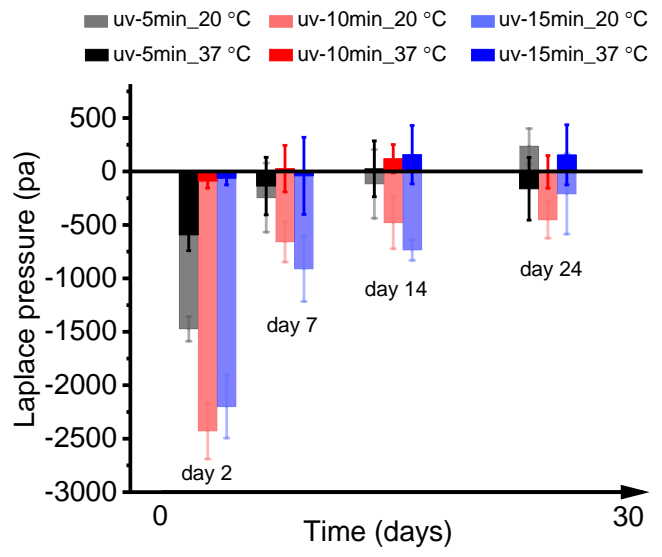

Figure 3.6. Calculated Laplace pressure based on figure 4 and equation 5 at $20^{\circ} \mathrm{C}$ and 37 ${ }^{\circ} \mathrm{C}$ for the indicated time. PNIPAm-g-PDMS channels prepared under UV irradiation times of 5, 10, $15 \mathrm{~min}$ are represented in black, red, and blue, respectively. The error bars represent the confidence interval of Laplace pressure calculated from the CA data from Figure 3.5.

Using the measured values of the static water CA on various channel $w$ alls, we can estimate the Laplace pressure in the channel using equation (1), to predict whether capillary filling of the channel will occur. As we can see from Figure 3.6, the CAs of freshly treated channels, using 5, 10,15 min UV exposure are all in a negative Laplace pressure range at both low and high temperatures. Freshly treated channels thus will alw ays generate a capillary driving force, and no valving action can be obtained by heating above the LCST. The Laplace pressure of the devices prepared with $5 \mathrm{~min}$ UV irradiation becomes positive after 24 days of storage, implying that capillary filling will not occur anymore at room temperature. Channels prepared under 10 and $15 \mathrm{~min} U V$ irradiation show larger Laplace pressure changes from $20^{\circ} \mathrm{C}$ and $37^{\circ} \mathrm{C}$ from negative to positive after 14 and 24 days and allow a better stopvalve function. As we didn't see a significant difference between devices prepared using 10 and 15 minutes irradiation, and longer UV irradiation time may make the surface opaque. ${ }^{41}$ Therefore, we performed surface grafting under $10 \mathrm{~min}$ UV exposure time. 


\subsubsection{Capillary filling behavior in the channel}

After grafting the PNIPAm, we observed the liquid filling process in freshly coated channels. Figure 3.7a shows the time-dependent filling in channels with the same width of $300 \mu \mathrm{m}$ and various heights of 34,60 , and $100 \mu \mathrm{m}$. The graphs show a constant filling velocity instead of a filling velocity decaying $w$ ith the square root of time as predicted by the classical Lucas-Washburn equation. ${ }^{2225}$ An average filling velocity of 160,217, $240 \mu \mathrm{m} / \mathrm{s}$ was obtained in the 34,60 and $100 \mu \mathrm{m}$ high channels, respectively. To better analyze the filling process, Figure 3.7b shows double-logarithmic plots of filling length against time. Thereby the red and blue dashed lines represent the extreme cases of diffusive hydrationlimited behavior (slope $=1$ ) and Lucas-Washburn behavior (slope $=2$ ), respectively. Slopes close to 1 were obtained for most filling processes, indicating that the diffusive hydration process dominates. In addition, an oscillating velocity at the contact line in themiddle part of the channel was observed (Video 1, 2, 3 in Appendix B show liquid filling behavior in 34,60, and $100 \mu \mathrm{m}$ high channels, respectively). As mentioned in the theoretical section, we attribute this phenomenon to the different wettability of the top and bottom walls in combination with transient contact line pinning. The pinning and depinning results in a periodical size change of the meniscus area, which is microscopically visible as a black area at the liquid front due to the angle that the meniscus surface makes with the normally incident light. When pinning occurs on the slowest moving contact line, the black area increases in size with the moving contact line on the opposite wall and reaches its maximum just before depinning of the slow contact line, followed by a sudden decrease when depinning occurs (slip jump), then reaching its minimum value. After a slip jump, the size of the black area increasing again until the next jump occurs. Since pinning and depinning are expected to occur randomly on all channel walls causing a complicated movement pattern, we only studied the size of the black area in the middle part of the channel to reduce the effect of pinning and depinning on sidew alls. Figure 3.7c shows the variation of the black area in time, the detailed measurement procedure is presented in 3.3.7. Periodical oscillations were observed with slip jump frequencies around 6.8, 4.4, and $4 \mathrm{~Hz}$. Due to the nonhomogeneous PDMS surface and the random growth of PNIPAm on the PDMS surface by UV-induced polymerization, the surface of PNIPAm-g-PDMS is nonhomogeneous, resulting in a less regular oscillation pattern during the filling process. Fur thermore, in Figure 3.7 a we see that a higher average velocity is obtained in the higher channel, different from the theoretical prediction of 
equation (6) that the velocity is independent of channel geometry. The reason could be that the dissipation caused by the distortion of the flow in the smaller channel is higher, as we see stronger periodical oscillations in this channel in

Figure 3.7c.
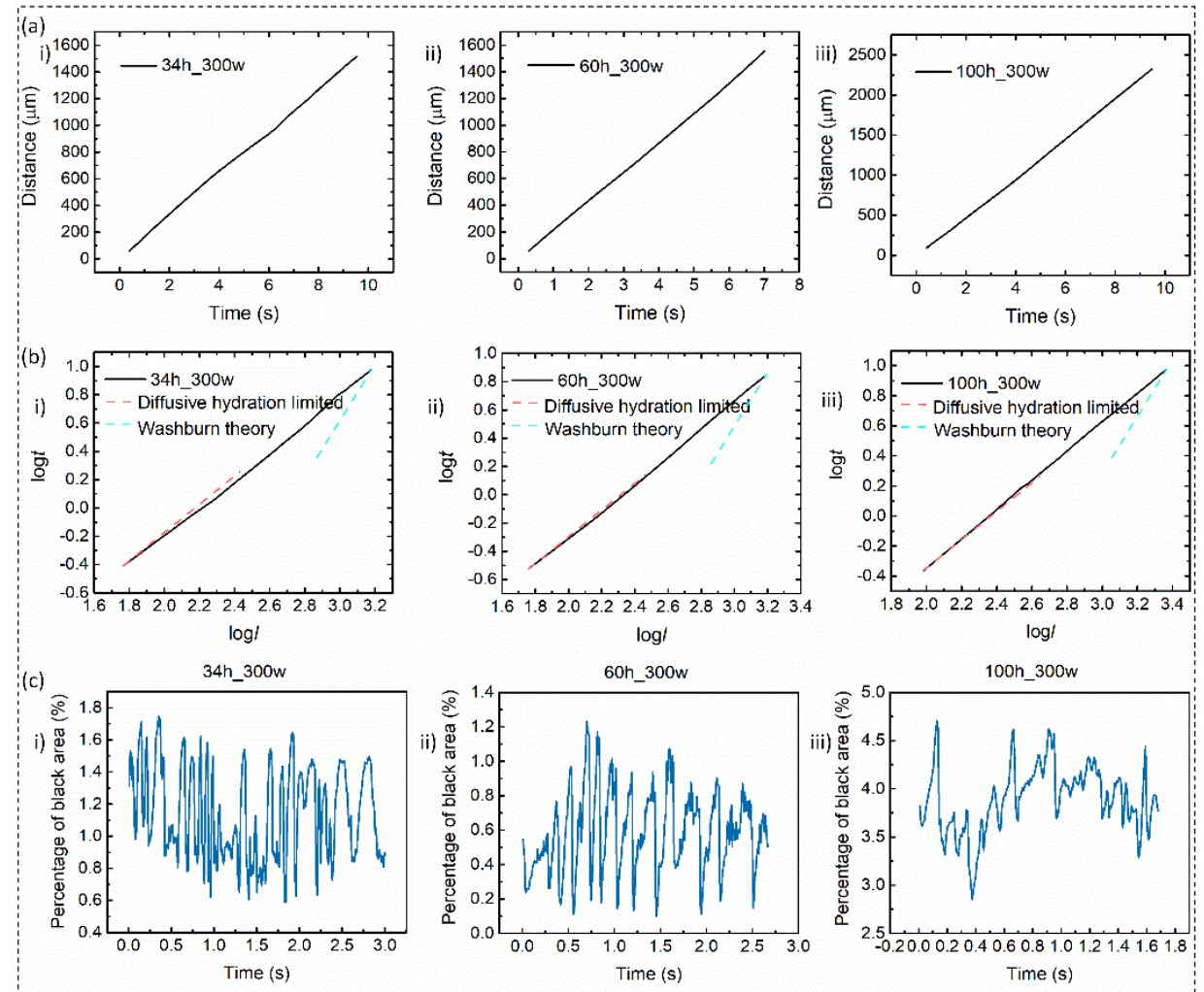

Figure 3.7. Liquid filling behavior in channels with different heights. (a) Filled length against time in channels of $300 \mu \mathrm{m}$ in width and 34 (i), 60 (ii), 100 (iii) $\mu \mathrm{m}$ in height. (b) Double-logarithmic plots of filling length against time. Channels were freshly coated, and the bottom walls are $60 \mu \mathrm{m}$ thick. (c) Percentage of the black area of the entire frame at the liquid front change in time in channels of 34 (i), 60 (ii), 100 (iii) $\mu \mathrm{m}$ high. The total area of the frames measured for (i), (ii), and (iii) are 35100, 46255, and $21056 \mu \mathrm{m}^{2}$, respectively.

\subsubsection{Heat-triggered valve action}

Due to the temperature response of the PNIPAm-g-PDMS surface, the channel w alls show a wettability change from hydrophilic to hydrophobic when their temperature changes from $20^{\circ} \mathrm{C}$ to $36^{\circ} \mathrm{C}$. By locally integrating a heatingelement in the bottom of the channel, a sw itchable stop valve w as obtained, open at low 
temperature, and closed at high temperature. Video 4 in appendix B shows the valving function. Figure 3.8 shows the meniscus position and velocity as well as snapshots of the filling process. Within the heating area, the liquid can be stopped and triggered by off-on heating cycles. The stop-valve function can only be obtained in devices that were stored for 14-24 days, where the Laplace pressuregenerated at the transition temperature of around $36^{\circ} \mathrm{C}$ was positive, as show $n$ in Figure 3.6. A possible disadvantage of using the devices after storage times of $\sim 20$ days is, that the filling velocity is below $10 \mu \mathrm{m} / \mathrm{s}$. How ever, this slow speed could be beneficial to achieve a homogeneous concentration of reactants by diffusion in chemical reactor chips ${ }^{23}$ or to allow long incubation times when performing immunoassays in microfluidicsystems. ${ }^{45}$

(a)

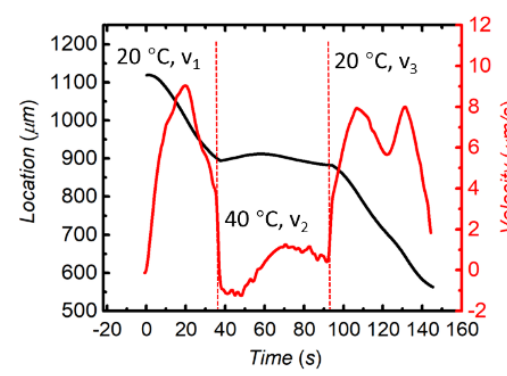

(b)

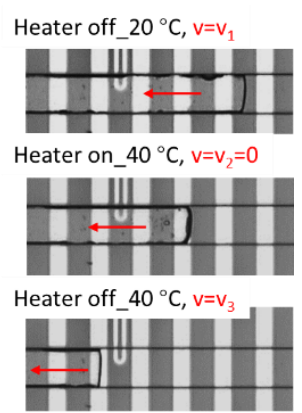

Figure 3.8. Controllable and reversible valving by locally varying the temperature between $20^{\circ} \mathrm{C}$ and $40^{\circ} \mathrm{C}$.

\subsection{CONCLUSION AND OUTLOOK}

We developed a PNIPAm-g-PDMS capillary microfluidic device that is characterized by a constant capillary flow rate and has integrated stop valves. Water fills the channel automatically by capillary forces and can be stopped by locally controlling the surface wettability of the channel walls by changing the temperature from $20^{\circ} \mathrm{C}$ to $36^{\circ} \mathrm{C}$. Constant flow velocities ranging from $1 \mu \mathrm{m} / \mathrm{s}$ to $240 \mu \mathrm{m} / \mathrm{s}$ can be obtained in dry PNIPAm-g-PDMS and freshly treated PNIPAmg-PDMS devices with different channel geometry. Stop and trigger valve function can be obtained by temperature control. Though it comes at the cost of a lower rate of filling, it could be beneficial to achieve a homogeneous concentration of the reactants by diffusion in reactions. ${ }^{23}$ The constant flow rate, obtained without external equipment of external flow control, $w$ as explained by diffusive hydration of the PNIPAm film. The theory is suitable for more 
complicated solutions and different channelgeometries. In this thesis, the change in velocity with channel height is not w ell explained, futurestudy of contact line motion in PNIPAm brush coated microfluidic channel using Molecular Dynamic simulations is recommended. In this chapter, the valving did not work in all devices due to variations in the water CA on the PNIPAm-g-PDMS surface at the hydrophobic state, sometimes causing an insufficient positive Laplace pressure. In chapter 5 , the problem has been solved by a surface with wider w ettability change between hydrophilic and hydrophobic states.

\subsection{REFERENCES}

1. Delamarche, E., Juncker, D. \& Schmid, H. Microfluidics for Processing Surfaces and Miniaturizing Biological Assays. Adv. Mater. 17, 2911-2933 (2005).

2. Hong, J., Edel, J. B. \& deMello, A. J. Micro- and nanofluidic systems for high-throughput biological screening. Drug Discov. Today 14, 134-146 (2009).

3. Dittrich, P.S. \& Manz, A. Lab-on-a-chip: microfluidics in drug discovery. Nat. Rev. Drug Discov. 5, 210-218 (2006).

4. Pihl, J., Sinclair, J., Karlsson, M. \& Orw ar, O. Microfluidics for cell-based assays Microfluidic systems are powerful tools in chemistry , physics, and. Mater. Today 8, 46-51(2005).

5. Gervais, L., de Rooij, N. \& Delamarche, E. Microfluidic Chips for Pointof-Care Immunodiagnostics. Adv. Mater. 23, H151-H176 (2011).

6. Woias, P. Micropumps - past, progress and future prospects. 105, $28-38$ (2005).

7. Eijkel, J. C. T. \& Van Den Berg, A. Young 4ever - The use of capillarity for passive flow handling in lab on a chip devices. Lab Chip 6, 1405-1408 (2006).

8. Lynn, N.S. \& Dandy, D. S. Passive microfluidic pumping using coupled capillary/evaporation effects. Lab Chip 9, 3422-3429 (2009).

9. Zimmermann, M., Schmid, H., Hunziker, P. \& Delamarche, E. Capillary pumps for autonomous capillary systems. Lab Chip 7, 119-125 (2007).

10. Olanrewaju, A., Beaugrand, M., Yafia, M. \& Juncker, D. Capillary microfluidics in microchannels: from microfluidic networks to capillaric circuits. Lab Chip 18, 2323-2347 (2018).

11. Feng, Y., Zhou, Z., Ye, X.\& Xiong, J. Passive valves based on hydrophobic microfluidics. in Sensors and Actuators, A: Physical 108, 138-143 (2003).

12. Zimmermann, M., Hunziker, P.\& Delamarche, E. Valves for autonomous capillary systems. Microfluid. Nanofluidics 5, 395-402 (2008). 
13. Lenk, G. A., Stemme, G. \& Roxhed, N. Delay valving in capillary driven devices based on dissolvable thin films. in 18th International Conference on Miniaturized Systems for Chemistry and Life Sciences, MicroTAS 2014 (2014).

14. Papadimitriou, V. A., Segerink, L. I., van den Berg, A. \& Eijkel, J. C. T. 3D capillary stop valves for versatile patterning inside microfluidic chips. Anal. Chim. Acta 1000, 232-238(2018).

15. Glière, A. \& Delattre, C. Modeling and fabrication of capillary stop valves for planar microfluidic systems. Sensors Actuators A Phys. 130-131, 601608 (2006).

16. Londe, G., Chunder, A., Wesser, A., Zhai, L. \& Cho, H. Microfluidic valves based on superhydrophobic nanostructures and switchable thermosensitive surface for lab-on-a-chip (LOC) systems. Sensors Actuators B Chem. 132,431-438(2008).

17. Chunder, A., Etcheverry, K., Londe, G., Cho, H. J. \& Zhai, L. Conformal switchable superhydrophobic/hydrophilic surfaces for microscale flow control. Colloids Surfaces A Physicochem. Eng. Asp. 333, 187-193(2009).

18. Yu, Q., Bauer, J. M., Moore, J. S. \& Beebe, D. J. Responsive biomimetic hydrogel valve for microfluidics. Appl. Phys. Lett. (2001). doi:10.1063/1.1367010

19. Eddington, D. T. \& Beebe, D. J. Flow control with hydrogels. Adv. Drug Deliv. Rev. (2004). doi:10.1016/j.addr.2003.08.013

20. Delaney, C. et al. Precision control of flow rate in microfluidic channels using photoresponsive soft polymer actuators. Lab Chip 17, 2013-2021 (2017).

21. Novo, P., Volpetti, F., Chu, V. \& Conde, J. P. Control of sequential fluid delivery in a fully autonomous capillary microfluidic device. Lab Chip 13, 641-645(2013).

22. Washburn, E. W. The Dynamics of Capillary Flow. Phys. Rev. 17, 273-283 (1921).

23. Juncker, D. et al. Autonomous microfluidic capillary system. Anal. Chem. 74,6139-6144(2002).

24. Tafti, E. Y. et al. Wettability control and flow regulation using a nanostructure-embedded surface. J. Nanosci. Nanotechnol. 11, 1417-1420 (2011).

25. Lucas, R. Ueber das Zeitgesetz des kapillaren Aufstiegs von Flüssigkeiten. Kolloid-Zeitschrift 23, 15-22 (1918).

26. Joos, P., Van Remoortere, P. \& Bracke, M. The kinetics of wetting in a capillary. J. Colloid Interface Sci. 136, 189-197 (1990).

27. Seebergh, J. E. \& Berg, J. C. Dynamic w etting in the low capillary number regime. Chem. Eng. Sci. 47,4455-4464(1992). 
28. Bracke, M., Voeght, F. \& Joos, P. The kinetics of wetting: the dynamic contact angle. Trends Colloid Interface Sci. III 149, 142-149(2007).

29. Hamraoui, A., Thuresson, K., Nylander, T. \& Yaminsky, V. Can a dynamic contact angle be understood in terms of a friction coefficient? J. Colloid Interface Sci. 226, 199-204(2000).

30. Mumley, T. E., Radke, C. J. \& Williams, M. C. Kinetics of liquid/liquid capillary rise. I. Experimental observations. J. Colloid Interface Sci. 109, 398-412(1986).

31. Yoon, J., Cai, S., Suo, Z. \& Hayw ard, R. C. Poroelastic swellingkinetics of thin hydrogel layers: comparison of theory and experiment. Soft Matter 6, 6004 (2010).

32. Silva, J. E. et al. Stick-slip water penetration into capillaries coated with sw elling hydrogel. Soft Matter 11,5933-5939 (2015).

33. Xia, Y. \& Whitesides, G. M. Soft lithography. Annu. Rev. Mater. Sci. 28, 153-184(1998).

34. Ebara, M., Hoffman, J. M., Hoffman, A. S. \& Stayton, P. S. Switchable surface traps for injectable bead-based chromatography in PDMS microfluidic channels. Lab Chip 6, 843 (2006).

35. Li, L. et al. In-Channel Responsive Surface Wettability for Reversible and Multiform Emulsion Droplet Preparation and Applications. ACS Appl. Mater. Interfaces 11, 16934-16943(2019).

36. Vollenbroek, J. C. et al. Design and characterization of a microreactor for monodisperse catalytic droplet generation at both elevated temperatures and pressures. in 2017 IEEE 12th International Conference on Nano/Micro Engineered and Molecular Systems (NEMS) 746-751 (IEEE, 2017). doi:10.1109/NEMS.2017.8017127

37. Otake, K., Inomata, H., Konno, M. \& Saito, S. Thermal Analysis of the Volume Phase Transition with N-Isopropylacrylamide Gels. Macromolecules 23, 283-289(1990).

38. Liu, Y. \& Sakurai, K. Thickness Changes in Temperature-Responsive Poly (N-isopropylacrylamide) Ultrathin Films under Ambient Conditions. ACS Omega 4, 12194-12203(2019).

39. Pelton, R. Poly(N-isopropylacrylamide) (PNIPAM) is never hydrophobic. J. Colloid Interface Sci. 348, 673-674(2010).

40. Hu, S. et al. Surface-Directed, Graft Polymerization within Microfluidic Channels. Anal. Chem. 76, 1865-1870(2004).

41. Ma, D., Chen, H., Shi, D., Li, Z. \& Wang, J. Preparation and characterization of thermo-responsive PDMS surfaces grafted with poly(N-isopropylacrylamide) by benzophenone-initiated photopolymerization. J. Colloid Interface Sci. 332, 85-90(2009). 
42. Hu, S. et al. Surface modification of poly(dimethylsiloxane) microfluidic devices by ultraviolet polymer grafting. Anal. Chem. 74, 4117-4123 (2002).

43. Ma, Y., Liu, L. \& Yang, W. Photo-induced living/controlled surface radical grafting polymerization and its application in fabricating 3-D microarchitectures on the surface of flat/particulate organic substrates. Polymer (Guildf). 52, 4159-4173(2011).

44. Ahmed, Z., Gooding, E. A., Pimenov, K. V., Wang, L. \& Asher, S. A. UV Resonance Raman Determination of Molecular Mechanism of Poly( N isopropylacrylamide) Volume Phase Transition. J. Phys. Chem. B 113, 4248-4256 (2009).

45. Ghodbane, M. et al. Development of a low-volume, highly sensitive microimmunoassay using computational fluid dynamics-driven multiobjective optimization. Microfluid. Nanofluidics 18, 199-214 (2015). 
Chapter 3 


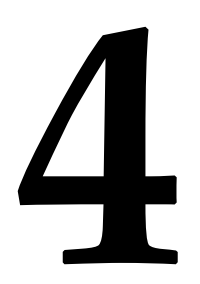

On-Chip Detection of the Alcohol Concentration in Beer 
Tn this chapter, w e investigated the wetting behavior of $w$ ater/ethanol mixtures

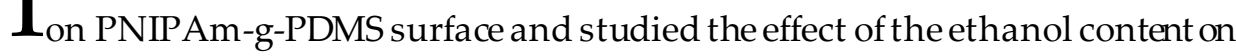
the filling behavior of water/ethanol mixtures in PNIPAm-g-PDMS microchannels. Water/ethanol mixtures between $0 \%$ to $4 \%$ molar fraction show a (non-W ashburn) constant filling flow rate in PNIPAm-g-PDMS microchannels with a flow rate that is dependent on the ethanol concentration. The dependence of the filling rate on the ethanol content makes the device a promising tool for the detection of low alcohol concentrations in alcoholic beverages by simply comparing the filling times. By using this method, Grolsch beers with $0 \%, 2 \%$, and $5 \% \mathrm{ABV}$ were distinguished from each other within $10 \mathrm{~s}$.

tThis chapter is based on the article manuscript: L. Li, L. Shui, M. Odijk, and J.C.T. Eijkel. On-chip detection of the alcohol concentration in beer. In preparation. 


\section{$4.1 \quad$ INTRODUCTION}

Ethanol is one of the main ingredients in beer, with a concentration ranging from $0.0 \%$ alcohol by volume (ABV) to over $10 \% .{ }^{1}$ In brewing industries, determination of ethanol in beer is important to control the quality of the beer. Classical methods to quantify the alcoholic content in alcoholic drinks are density measurements using a density meter, pycnometer, or hydrometer. These methods however lack rapidity and sensitivity. ${ }^{2}$ Other techniques using chromatographic methods such as high-performance liquid chromatography (HPLC) $)^{3,4}$ or gas chromatography (GC), ${ }^{5}$ show the advantages of selectivity and high speed of analysis but are often too expensive for small companies. Spectrometric analyses such as infrared (IR) spectrometry, ${ }^{6}$ spectrophotometric, 7 nuclear magnetic resonance (NMR) spectroscopy, ${ }^{8}$ and Raman spectroscopy 9,10 offer alternative methods to quantify ethanol concentrations in alcoholic beverages but also rely on more expensive equipment. New methods with rapidity and high sensitivity are therefore still in demand for the (home) brewery industry and laboratories.

In recent years, promising sensing approaches based on poly $(\mathrm{N}$ isopropylacrylamide) (PNIPAM)-based stimuli-responsive hydrogels for the detection of ethanol have been reported, ${ }^{11,12}$ characterized by a sensitive, facile, and rapid detection. Crosslinked PNIPAm and PNIPAm-copolymer gels undergo a volume-phase transition due to the co-nonsolvency effect in w ater/alcohol mixtures, where the microgel particle size or gel thickness varies with the alcohol concentration. ${ }^{13,14}$ Based on the swelling behavior of PNIPAm hydrogel in water/ethanol mixtures, ethanol sensors based on a piezoresistive pressure change ${ }^{11}$ and a diffraction efficiency (DE) change ${ }^{15}$ have been reported. Alcohol concentrations between $10 \%-50 \%$ were determined. These methods offer new potential for industry applications. How ever, a rapid and accurate method for the determination of ethanol concentrations below $10 \%$ is still needed.

In chapter 3, we developed a PNIPAm grafted PDMS (PNIPAm-g-PDMS)-based capillary microfluidic device that is characterized by a water filling rate that is constant in time. The constant filling rate was attributed to the diffusive hydration of the PNIPAm film in water at the liquid front. In literature, the w ater/PNIPAm interaction can be affected by the addition of a small amount of alcohol. In this chapter, w e investigated the effect of the ethanol content of water on the w etting behavior of PNIPAm-g-PDMS surfaces and the filling behavior of PNIPAm-g-PDMS microchannels. We found that $\mathrm{w}$ ater/ethanol mixtures with 
an ethanol molar fraction between $0 \%$ to $4 \%$ have a constant, ethanol concentration-dependent filling rate. This makes the device a promising tool for the detection of low alcohol concentrations in alcoholic beverages by simply comparing the filling time of the microchannels. The device was then used for the detection of low alcohol concentrations in Grolsch beer. Different beer types with alcohol concentrations from 0 to $5 \% \mathrm{ABV}$ were tested and could be distinguished from each other within $10 \mathrm{~s}$.

\subsection{WORKING PRINCIPLE}

4.2.1 Phase behavior of Poly(Nisopropylacrylamide) in w ater/ethanol mixtures

Poly (N-isopropylacrylamide) (PNIPAm) is a widely studied thermo-responsive polymer. The PNIPAm polymer chain undergoes a reversible coil-to-globule transition at around $32{ }^{\circ} \mathrm{C}$ in aqueous solutions. ${ }^{3}$ This temperature is known as its low er critical solution temperature (LCST). At temperature below its LCST, PNIPAm is soluble in water, forming a one phase transparent solution. At temperature above its LCST, the polymer chain precipitate from the solution. The temperature-induced phase separation of PNIPAm solutions is attributed to the dehydration of the PNIPAm molecules. ${ }^{16-18}$ Besides its thermo-responsive behavior, PNIPAm is also sensitive to the solvent composition such as wateralcohol mixtures. At a fixed temperature, e.g. $T=20^{\circ} \mathrm{C}$, PNIPAm dissolves in both pure water and pure methanol. However, the polymer chain exhibits a Coilto-Globule-to-Coil transition in water/methanol mixture with increasing methanol content (Figure 4.1a ${ }^{19}$ ), resulting in phase separation of PNIPAm in the w ater/methanol mixture. As a result, the LCST of a PNIPAm solution decreases with increasing methanol content of a water-rich zone and PNIPAm becomes insoluble in an intermediate range of solvent compositions, while with further enrichment of the methanol content, the polymer becomes soluble again in the solvent mixture. This phenomenon is called cononsolvency. ${ }^{20,21}$ So far, the cononsolvency phenomenon of PNIPAm-water-methanol solutions has been widely studied based on experimental studies and simulations. Theories on the co-nonsolvency, such as solvent-complex, ${ }^{2223}$ competitive hydrogen bonding, ${ }^{2426}$ preferential absorption ${ }^{2728}$ and strong water-cosolvent interactions ${ }^{29}$ have been reported. It has for example been reported that, in a w ater-rich solvent mixture, the presenceof methanol results in a weakening of PNIPAm-Solvent interaction, inducing a decrease in the number of hydrogen bond between PNIPAm and the solvents. ${ }^{19}$ How ever, the mechanisms underlying co-nonsolvency are still under debate. Few studies focusing on the co-nonsolvency of PNIPAm in water/ethanol 
mixtures have been reported, where results show that the reduction of the molar partial enthalpy of $w$ ater $w$ ith increasing ethanol content is the key factor of the LCST change in water-PNIPAm-ethanol solutions. ${ }^{30,31}$ Although the theory behind the co-nonsolvency of PNIPAm in water/alcohol mixtures is not fully understood, experimental results are in good agreement. Figure $4.1 b^{30}$ shows the phase behavior of PNIPAm with different viscosity averaged molecular weight $\left(M_{v}\right)$ and concentration $(c)$ in w ater/ethanol mixtures at different ethanol molar fractions in the temperature range of $-20-40^{\circ} \mathrm{C}$.

(a)

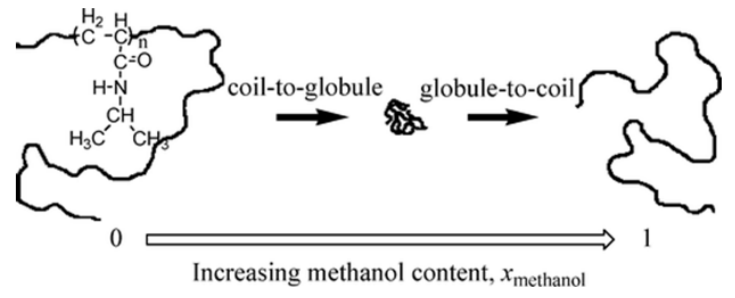

(b)

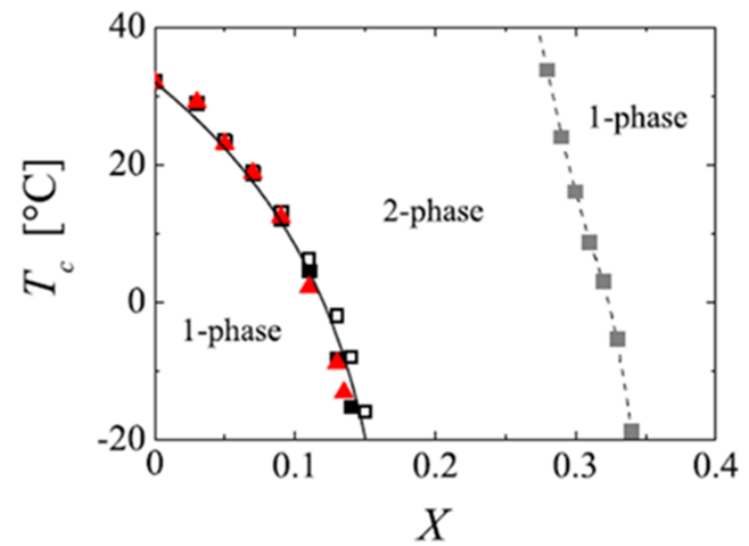

Figure 4.1 (a) Coil-to-Globule-to-Coil transition of a PNIPAm chain with increasing methanol content, $x_{\text {methanol, in water/methanol mixtures at room temperature. Reprinted }}$ with permission from Pang et al. ${ }^{19}$ Copyright 2010 American Chemical Society. (b) The relationship between the critical solution temperature $T_{c}$ and the ethanol molar fraction $X$. The full squares and open squares represent data for a PNiPAM with $M v=39000 \mathrm{~g} / \mathrm{mol}$ at concentrations of $\mathrm{c}=10^{-2} \mathrm{~g} / \mathrm{ml}$ and $\mathrm{c}=2 \cdot 10^{-3} \mathrm{~g} / \mathrm{ml}$, respectively. The full triangles represent data for a solution of a PNiPAM with $M v=465500 \mathrm{~g} / \mathrm{mol}$ at a concentration of c $=10^{-2} \mathrm{~g} / \mathrm{ml}$. Figure adapted with permission from Bischofberger et al. ${ }^{30}$ Copyright 2014 Springer Nature.

Figure 4.1b presented the phase behavior of PNIPAm in water/ethanol mixtures. PNIPAm is soluble in both pure water and ethanol at room temperature, while 
its solubility in water/ethanol mixtures is dependent on the ethanol content. In water-rich mixture with $X<0.15$, PNIPAm is fully soluble in water/ethanol mixtures at temperatures below the LCST. The polymer chain collapses with increasing $X$. When the molar ethanol fraction is increased to about 0.15 , the PNIPAM chains experience a coil-to-globule transition which results in phase separation. The chains stay in a globular state in the molar fraction range from 0.15 to 0.34 . Above $X=0.34$, the polymer becomes soluble again at room temperature in ethanol-rich mixtures.

The phase behavior of PNIPAm polymer solutions in response to temperature and water-alcohol content is reflected in PNIPAm brushes, ${ }^{32,33}$ PNIPAm thin films ${ }^{34}$ and PNIPAm-based crosslinked gels, ${ }^{35,36}$ which undergo a brush or gel sw elling in response to compositional variations in the w ater-alcohol mixture. In chapter 3, we attributed the capillary filling behavior of pure w ater in PNIPAmg-PDMS microchannel to the diffusive hydration of the PNIPAm film. Based on the co-nonsolvency of PNIPAm in a water/ethanol mixture, the hydration process of PNIPAm in the liquid mixture will change with the solvent composition, and capillary filling behavior of the solvent mixture in a PNIPAmg-PDMS microchannel will be solvent composition dependent.

4.2.2 Capillary filling behavior of $w$ ater/ethanol mixtures in a PNIPAm-g-PDMS microchannel

The capillary filling process of a rectangular microfluidic channel with width $w>>$ height $h$ can be described by the Lucas-Washburn equation ${ }^{37,38}$, balancing the driving force resulting from the surface tension and the resistive viscous force.

$$
2 w \gamma / \mathrm{lv} \cos \theta_{\mathrm{s}}=\frac{12 \eta w}{h} l(\mathrm{t}) \frac{d l(\mathrm{t})}{d t}
$$

Here $\gamma_{\mathrm{lv}}(\mathrm{N} / \mathrm{m})$ is the surface tension of the liquid, $\theta_{\mathrm{s}}\left(^{\circ}\right)$ is the static liquid/solid contact angle (CA) in the channel, $l(\mathrm{t})(\mathrm{m})$ is the filling length of liquid within the filled channel section at time $t(\mathrm{~s})$, and $\eta\left(\mathrm{N} \cdot \mathrm{s} \cdot \mathrm{m}^{-2}\right)$ is the dynamic viscosity of the liquid.

In equation (1), it is assumed that the contact angle $\theta_{\text {s remains constant during }}$ the filling process. The filling behavior is then characterized by $l(t) \alpha \sqrt{t}$. How ever, in PNIPAm-g-PDMS channel, a dynamic wetting of $w$ ater of PNIPAm w as presented in chapter 3 , a constant capillary filling rate $(l(t) \alpha t)$ w as obtained. We ascribed this phenomenon to the slow diffusive hydration process of the PNIPAm chains at the contact line, which could be described by a dynamic 
contact angle. In our system, the dynamic contact angle $\theta_{d}$ w as approximated with an equation combining the diffusionalhydration velocity $\alpha D / d$ at the liquid front and the meniscus velocity $d l(t) / d t$,

$$
\cos \theta_{d}=\cos \theta_{s}\left(1-\frac{d}{\alpha D} \cdot \frac{d l(t)}{d t}\right)
$$

Here $\alpha$ is a numerical constant with a value between 2 (1D diffusion) and 4 (2D diffusion), $D$ is the diffusion coefficient of w ater in a PNIPAm brush and $d$ is the diffusion length in front of the liquid meniscus.

From equations 1 and 2 we obtain

$$
\gamma l v \cos \theta_{s}\left(1-\frac{d}{\alpha D} \cdot \frac{d l(t)}{d t}\right)=\frac{6 \eta}{h} l(t) \frac{d l(t)}{d t}
$$

Solving equation 3 we obtained

a. When $d l(t) / d t \approx \alpha D / d$, the diffusive hydration of w ater on PNIPAm-g-PDMS surface limits the filling process, and the filling length $l$ will be proportional to time $t$

$$
l(t)=\frac{\alpha D}{d} t
$$

b. When $d l(t) / d t \ll \alpha D / d$, we retrieve the Lucas-Washburn equation, with $l$ proportional to the square root of time $(\sqrt{t})$,

$$
l(\mathrm{t})=\sqrt{\frac{h \gamma_{\mathrm{w}} \cos \theta_{s} t}{3 \eta}}
$$

Equations 4 and 5 describe the capillary filling of water in PNIPAm-g-PDMS microchannel. When the diffusive hydration process dominates, the filling rate will be constant in time, whilst when the diffusive hydration can be neglected, the filling process follows Lucas-Washburn theory and willbejointly determined by the surface tension of the liquid, the dynamic viscosity, the contact angle of liquid on the surface and the channel geometry.

Based on the considerations above, the capillary filling behavior of water/ethanol mixture in PNIPAm-g-PDMS microchannel may show a sensitivity to the ethanol content in water/ethanol mixture. This is investigated in this chapter. 


\subsection{MATERIALS AND METHODS}

\subsubsection{Materials}

N-Isopropylacrylamide (NIPAm, CAS: 2210-25-5), benzophenone (CAS: 119-619), $\mathrm{NaIO}_{4}$ (CAS: 7790-28-5), and benzyl alcohol (CAS: 100-51-6) were all obtained from Sigma-Aldrich (the Netherlands). Negative photoresist SU-8 3050 and developer solution were purchased from MicroChem (MA, USA) for fabricating the mold with the designed microchannelsin SU-8 on a Si w afer ( $525 \mu \mathrm{m}$ thick, Okmetic, Finland). The poly (dimethylsiloxane) (PDMS, Sylgard 184) package w as purchased from Dow Corning Corporation (Midland-Michigan, USA) and was used for fabricating the microfluidic chip.

\subsubsection{PDMS device fab rication}

The poly dimethylsiloxane (PDMS) microfluidic devices were fabricated using standard photolithography techniques. ${ }^{39}$ The detailed fabrication process was presented in chapter 3 .

4.3.3 UV-induced surface grafting of NIPAm on PDMS mediated by benzophenone

UV-induced grafting of PNIPAm was conducted by UV-initiated surface polymerization ${ }^{40}$. The detailed grafting method was presented in chapter 3 .

\subsubsection{Contact angle measurements}

A single drop of a water/ethanol mixture with a small volume (2-12 $\mu \mathrm{L})$ was dripped gently on the PNIPAm-g-PDMS substrates. An interfacial tension meter OCA 15 Pro (Dataphysics, Germany) was used to measure the static contact angle or dynamic contact angle of ethanol-water and beer samples on PNIPAm-gPDMS at room temperature.

\subsubsection{Capillary filling measurement procedure and data processing}

Before the filling process, PDMS chips w ere grafted with PNIPAm and washed thoroughly with DI water. After drying by air blowing, the chip was then used to study the filling process of water/ethanol mixtures or beer samples. For this purpose, $10 \mu \mathrm{L}$ of water/ethanol mixture or beer sample was pipetted in the reservoir. To check the reproducibility, each sample was tested at least 3 times in the same chip. Before filling the samples, the channel $w$ as $w$ ashed with DI $w$ ater and dried by blowing air. The filling behavior of the channels $w$ as observed 
using a Leica DM 6000 M microscope with a Leica DFC 420 digital camera (Wetzlar, Germany). Captured pictures and videos were analyzed using Matlab R2019a and ImageJ (version: 2.0.0-rc-69/1.52p) to calculate the location of the liquid contact lines in the channel in time. Detailed data processing methods were presented in chapter 3 .

\subsection{RESULTS AND DISCUSSION}

\subsubsection{Wetting behavior of w ater/ethanol mixtures on PNIPAm-g-PDMS}

The w etting behavior of DI w ater, water-ethanol mixtures with increasing molar fraction, and pure ethanol have been studied by CA measurements. Results are shown in Figure 4.2a. CAs of these liquid solutions on bare PDMS have been measured (Figure $4.2 \mathrm{~b}$ ) as references.
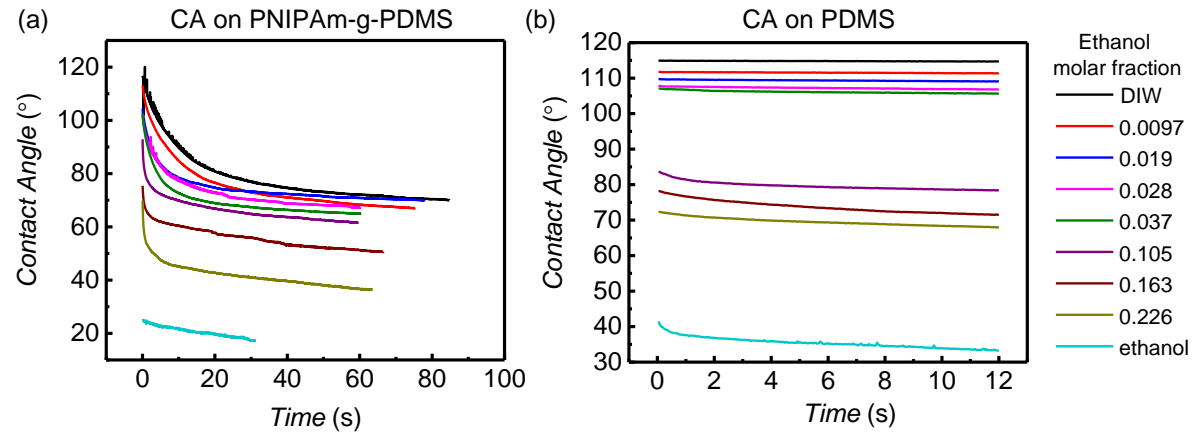

Figure 4.2 Wetting behavior of DI water, water-ethanol mixtures with different ethanol molar fractions and pure ethanol on PNIPAm-g-PDMS (a) and bare PDMS (b). CAs are measured at a temperature of $20^{\circ} \mathrm{C}$.

As seen from Figure 4.2a, DI w ater and water-ethanol mixtures w ith increasing molar fraction as well as pure ethanol all show time-dependent wetting on PNIPAm-g-PDMS. With increasing ethanol molar fraction, this time-dependent wetting behavior becomes weaker compared to pure water. When $X$ is in the range of $0.01-0.04$, it takes more than 20s toreach a static $C A$, while in the higher molar fraction range $(X>0.1)$, the $C A$ reaches the static state within a few seconds. What we need to remark here is that the continuously decreasing CAs at longer times are due to evaporation, especially for those solutions with higher $X$, as we saw the changes in droplet profile without baseline changes when we measured the CAs. The difference in the wetting behavior of these liquid solutions on a PNIPAm-g-PDMS surface and a bare PDMS surface were measured and are shown in Figure 4.2b. Pure water and water/ethanol mixtures with lower ethanol content $(0.0097<X<0.037)$ show no time-dependent wetting behavior on a 
PDMS surface butCAs $>90^{\circ}$ due to the hydrophobic nature of PDMS. How ever, w ater/ethanol mixtures with $X>0.1$ show dynamic wetting at the very beginning of the w etting state (micro-second scale), which may be due to the preference of ethanol for hydrophobic surfaces. ${ }^{41}$ We also studied the wetting behavior of w ater/ethanol mixtures ( $X=0.0097,0.028,0.037,0.226$ ) and pure ethanol on PNIPAm-g-PDMS at different temperatures $\left(20-36^{\circ} \mathrm{C}\right)$ (Figure $\mathrm{C} 1$ in Appendix C). An increasing contact angle of $w$ ater/ethanol mixtures $w$ ith temperature was observed at $T<32{ }^{\circ} \mathrm{C}$. From $T=32{ }^{\circ} \mathrm{C}$ to $T=36{ }^{\circ} \mathrm{C}$, w ater-ethanol mixtures with an $X$ of 0.226 and ethanol show a decrease in contact angle. The different temperature-dependent wetting behavior may be due to the transition from solvent-polymer interaction domain to solvent mixing domain with increasing $X .42$

Based on the co-nonsolvency phenomenon of PNIPAm mentioned in the literature cited in section 4.2.1, we make a hypothesis that a) the stronger timedependent wetting behavior of water/ethanol mixtures with lower ethanol content $(0.0097 \leq X \leq 0.037)$ at $20^{\circ} \mathrm{C}$, is due to the swelling of PNIPAm in the liquid, which is similar to the wetting behavior of $w$ ater on a PNIPAm-g-PDMS surface. From $X=0$ to $X \approx 0.1$, increasing $X$ values result in lower swelling rates of PNIPAm, hence we see the CAs of water/ethanol mixtures with higher $X$ reach the static CAs faster. The minimum swelling rate is reached between $X \approx 0.1$ and $X \approx 0.226$. When $0.1 \leqslant X \leqslant 0.226$, the PNIPAm forms a globular structure in the solution at room temperature, making the surface a hard surface where the diffusive hydration can be neglected. To our knowledge, the wetting behavior of w ater/ethanol mixtures on PNIPAm-g-PDMS have not been reported before. Our experimental results and hy pothesis match the swelling behavior of PNIPAm brushes in water/ethanol mixture reported by Yu et al. ${ }^{33}$ very well, where the brush collapsed for ethanol volume fractions between 20 and $50 \%$, which is the molar fraction between $0.07-0.24$.

4.4.2 Capillary filling behavior of $w$ ater/ethanol mixtures in PNIPAm-g-PDMS microchannels

We further investigated the capillary filling behavior of $w$ ater/ethanol mixtures in PNIPAm-g-PDMS microchannels. Figure 4.3 shows how the capillary filling distance of different solutions in time. Double logarithmic plots $(\log x-\log t)$ have been added to better clarify the filling behavior. A slope $=1$ demonstrates diffusive hydration making the filling flow rate constant in time (equation (4)). A 
slope $=2$ demonstrates Lucas-Washburn behavior with a filling distance increasing with $\sqrt{t}$ (equation (5)).
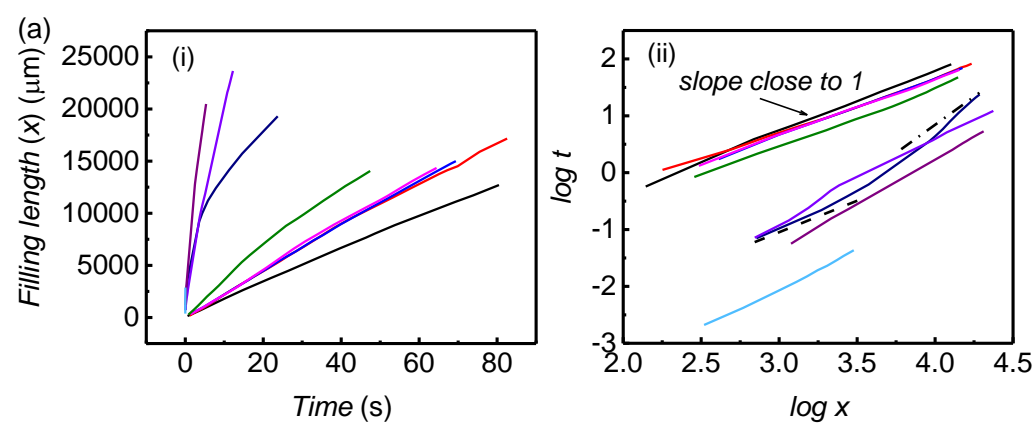

Ethanol molar fraction

- DIW

0.0097

0.019

0.028

0.037

0.105

$-0.163$

0.226

ethanol

Time (s)

$-\cdot-\cdot$ slope $=2$
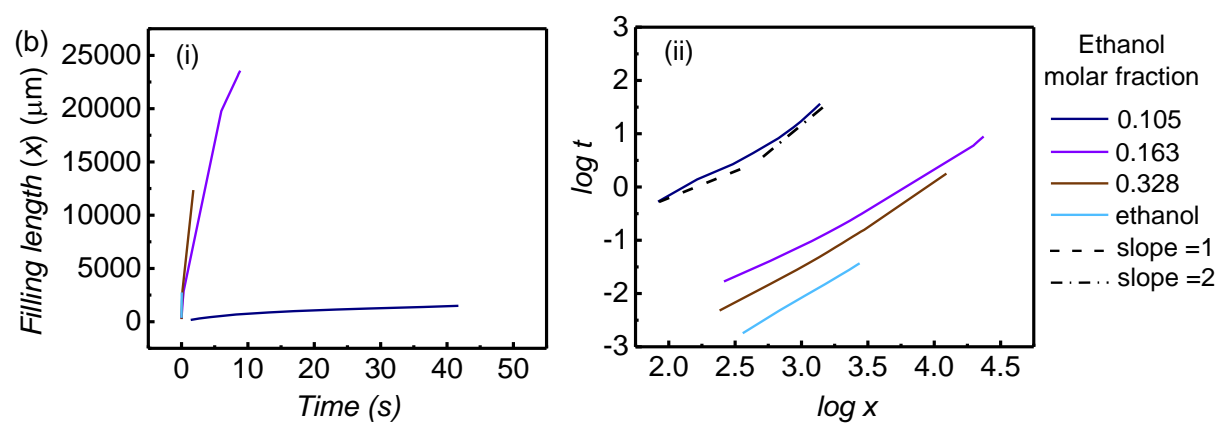

Figure 4.3 Capillary filling behavior of DI water, water-ethanol mixtures, and pure ethanol in PNIPAm-g-PDMS microchannels (a) and PDMS microchannels (b). The dimensions of the microchannels are $600(w) \mu \mathrm{m} \times 100(h) \mu \mathrm{m} \times 25000(l) \mu \mathrm{m}$.

The DI w ater, as well as water/ethanol mixtures with $X$ of $0.0097 \leq X \leq 0.028$ in

Figure 4.3a, shows a constant capillary filling flow rate with a slope in the double logarithmic plot close to 1 (Figure 4.3a (ii)). The filling flow rates of water/ethanol mixtures show a clear difference $w i t h$ that of DI $w$ ater and a slight dependence on $X$. A slightly nonlinear $\log t-\log x$ relationship was observed in a w ater/ethanol mixture of $X=0.037$. When $X=0.105$, a clear transition from a slope of 1 to a slope of $2 \mathrm{w}$ as observed, indicating that the filling behavior changed from diffusive hydration to Washburn behavior. When further increasing the ethanol content, e.g. $X=0.163$ and $X=0.226$, the capillary filling behavior of the water/ethanol mixture is similar to that of pure ethanol in a PNIPAm-g-PDMS microchannel, with a slope of $\log t-\log x$ plot close to 1.5. Such a filling behavior is comparable to the filling behavior of an ethanol-rich mixture in a PDMS microchannel. Taking into account the dynamic wetting behavior of ethanol-rich w ater/ethanol mixtures on PNIPAm-g-PDMS and bare PDMS surfaces in Figure 4.2, a dynamic capillary filling in PNIPAm-g-PDMS and bare PDMS 
microchannel w ould exist at the beginning of the filling process and may show a transition of filling behavior to Washburn behavior after some time. In our experiment, since the fillingspeed of the ethanol rich mixture is very high $(\mathrm{mm} / \mathrm{s})$, we can only capture the very beginning of the filling process $(t<1 \mathrm{~s})$, so we can't see a transition in Figure 4.3. In our experiment, we repeated the measurement in different devices with identical geometry, qualitatively identical (diffusive hydration-determined or Washburn behavior) filling behavior was observed, though the slopes might slightly differ.

On the basis of literature and our CA measurements we interpret the experimental results obtained as follows. When filling the channel with pure water, the diffusive hydration is dominated by the water-amide group interaction ${ }^{15}$. In the presence of ethanol, the interaction between water and ethanol is enthalpically favorable for $w$ ater, ${ }^{42}$ inducing the partial collapse of PNIPAm brushes in a water-rich water/ethanol mixture. In water/ethanol mixtures with an ethanol molar fraction $X<0.05$, water will form a "cage" surrounding the ethanol. ${ }^{43}$ There is no apparent collapse of the PNIPAm chain how ever, thus we expect that in our system the filling behavior is still dominated by diffusive hydration, showing a constant filling rate. In addition, the interaction between PNIPAm and ethanol is mainly due to the van der Waals interactions between isopropyl and ethyl groups, ${ }^{31}$ the electrostatic interaction (dipole-dipole interaction) between ethanol and the PNIPAm increases with the increase in ethanol concentration, ${ }^{44}$ resulting in continuous adsorption at the liquid front on the PNIPAm-g-PDMS channel surface, which contributes to the filling velocity. Hencewe see the filling flow rate increase slightly with increasing ethanol content for $0.0097 \leqslant X \leqslant 0.037$. When $0.105 \leqslant X \leqslant 0.226$, the collapse of the PNIPAm brush forms a hard surface, and no diffusional hydration occurs. The filling behavior now follows the Washburn theory. Finally, when filling the channel with pure ethanol, due to the good solvency of ethanol and the nonspecific PNIPAm-ethanol interactions, ${ }^{42,44}$ we don't have a slow dynamic wetting anymore (Figure 4.2a), the filling behavior of ethanol follows Washburn's theory.

4.4.3 Capillary filling behavior of Grolsch beer in PNIPAm-g-PDMS microchannel

Since water/ethanol mixtures with low ethanol molar fraction show constant filling behaviors in PNIPAm-g-PDMS channels with a sensitivity to the ethanol content, we decided to further study the filling behavior of real beer in PNIPAm- 
g-PDMS microchannels. Grolsch beers with alcohol contents of $0.0 \%$ (Grolsch $0.0 \%$ ), $2.0 \%$ (Grolsch - Radler) and $5.0 \%$ (Grolsch - Premium) were chosen as samples. Results are shown in Figure 4.4. Grolsch beers in PNIPAm-g-PDMS channel show a filling flow rate increasing with the increase in alcohol content. Figure 4.4a shows the filling distance $(x)$ of different Beers in the channel with time $(t)$, and Figure $4.4 \mathrm{~b}$ is the double logarithmic plots of $x$ and $t$ to better classify the filling behavior of real beers in the channel. As we can see from the results, non-alcohol beer $(0.0 \% \mathrm{vol})$ exhibits a nearly constant flow rate with $\log x$ and $\log t$ shows a linear relationship with a slope close to 1 in Figure 4.4b. Filling behaviors of $2 \%$ vol beer shows a filling flow rate slightly decrease with time with filling length proportional to $t^{0.9}$ (Figure $4.4 \mathrm{a}$ ), indicating a flow rate that is not so constant. From the filling behavior of $5.0 \%$ beer in Figure $4.4 \mathrm{~b}$, we see a clear transition from $\log t-\log x$ plot with slope change from 1.1 to 0.72 . The different filling behavior of real beer compare with water/ethanol mixture may due to the high viscosity of the beer sample, resulting in a transition from diffusion hydration dominant to Washburn behavior (surface tension and viscosity dominant). The slope of $0 \%, 2 \%$, and $5 \%$ ABV can be distinguished from each other within $10 \mathrm{~s}$.

(a)

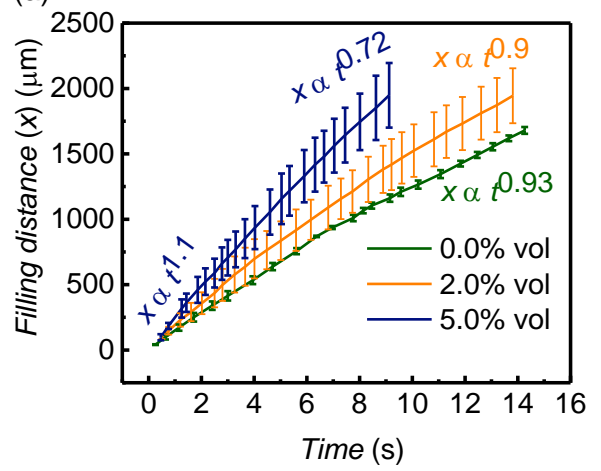

(b)

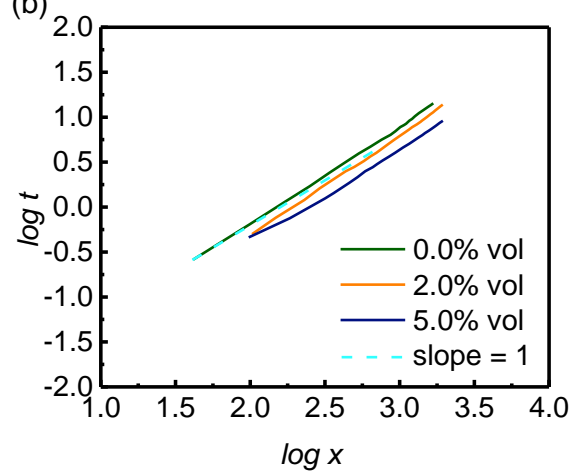

Figure 4.4 Capillary filling behavior of different types of Grolsch beers with an alcohol content of $0.0 \%, 2.0 \%$, and $5.0 \%$ ABV in a PNIPAm-g-PDMS microchannel. (a) Filling distance $(x)$ of different beers in the channel with time $(t)$, (b) Double logarithmic plots of $x$ and $t$. The errorbars represent the standard deviation of filling distance calculated from three repeated experiments in the same channel.

\subsection{CONCLUSION AND OUTLOOK}

In this chapter, we investigated the wetting behavior of w ater/ethanol mixture on PNIPAm-g-PDMS surfaces and the capillary filling behavior of w ater/ethanol mixtures in PNIPAm-g-PDMS microchannels. Water/ethanol mixtures with low 
ethanol molar fraction $(0.0097<X<0.037)$ show a strong time-dependent wetting on PNIPAm-g-PDMS surfaces and constant filling flow rate in PNIPAm-g-PDMS microchannels. The filling speed is increasing with ethanol content. Based on the sensitivity of the filling flow rate to the ethanol content in water/ethanol mixtures, Grolsch beers with an alcohol content of $0.0 \%, 2.0 \%$, and $5.0 \%$ ABV can be distinguished from each other in $10 \mathrm{~s}$. This shows the potential utility of PNIPAm-g-PDMS microfluidic devices for sensing low alcohol concentrations in solution, e.g. alcoholic beverages, blood samples, and sweat.

Apart from alcohol content and temperature, also ions such as Hofmeister Series ${ }^{45}$ have an influence on the solubility and chain for mation of PNIPAm in an aqueous solution and could be sensed. Furthermore, in this chapter, we only studied the wetting behavior at $T>20^{\circ} \mathrm{C}$. How ever, the LCST of PNIPAm in w ater-rich ethanol-water mixtures (e.g. of $0.05<X<0.15$ ) is below $20^{\circ} \mathrm{C}$. Future studies on the effect of ions and a wider temperature range on the filling behavior of $w$ ater/ethanol mixtures are recommended for practical sensing purposes.

\subsection{REFERENCES}

1. Rajendram, R. \& Preedy, V. R. Ethanol in Beer: Production, Absorption and Metabolism. in Beer in Health and Disease Prevention 431-440 (Elsevier, 2009). doi:10.1016/B978-0-12-373891-2.00041-9

2. Tonelli, D. Methods for Determining Ethanol in Beer. in Beer in Health and Disease Prevention 1055-1065 (Elsevier, 2009). doi:10.1016/B978-012-373891-2.00102-4

3. Lopez, E. F. \& Gomez, E. F. Simultaneous Determination of the Major Organic Acids, Sugars, Glycerol, and Ethanol by HPLC in Grape Musts and White Wines. J. Chromatogr. Sci. 34, 254-257 (1996).

4. Yarita, T. et al. Determination of ethanol in alcoholic beverages by highperformance liquid chromatography-flame ionization detection using pure water as mobile phase. J. Chromatogr. A 976, 387-391 (2002).

5. Wang, M.-L., Choong, Y.-M., Su, N.-W. \& Lee, M.-S. A rapid method for determination of ethanol in alcoholic beverages using capillary gas chromatography. J. Food Drug Anal. 11, 133-140 (2003).

6. Lachenmeier, D. W. et al. Rapid and mobile determination of alcoholic strength in wine, beer and spirits using a flow -through infrared sensor. Chem. Cent. J.4, 5 (2010).

7. SASAKI, Y., TAGASHIRA, S., MURAKAMI, Y. \& KAI, S. Analytical Application of O,O'-dialkyl dithiophosphate IX. Spectrophotometric 
Determination of the Alcohol Content of Alcoholic Drinks with Bis(O,O'dipropyl dithiophosphato)nickel(II). Anal. Sci. 9, 483-486(1993).

8. Isaac-Lam, M. F. Determination of Alcohol Content in Alcoholic Beverages Using 45 MHz Benchtop NMRSpectrometer. Int. J. Spectrosc. 2016, 1-8(2016).

9. Cleveland, D. et al. Raman Spectroscopy for the Undergraduate Teaching Laboratory: Quantification of Ethanol Concentration in Consumer Alcoholic Beverages and Qualitative Identification of Marine Diesels Using a Miniature Raman Spectrometer. Spectrosc. Lett. 40, 903924 (2007).

10. Boyaci, I. H., Genis, H. E., Guven, B., Tamer, U. \& Alper, N. A novel method for quantification of ethanol and methanol in distilled alcoholic beverages using Raman spectroscopy. J. Raman Spectrosc. 43, 1171-1176 (2012).

11. Erfkamp, J., Guenther, M. \& Gerlach, G. Hydrogel-based piezoresistive sensor for the detection of ethanol. J. Sensors Sens. Syst. 7, 219-226(2018).

12. Erfkamp, J., Guenther, M. \& Gerlach, G. Hydrogel-based sensors for ethanoldetection in alcoholic beverages. Sensors (Switzerland) 19, (2019).

13. Althans, D., Langenbach, K. \& Enders, S. Influence of different alcohols on the swelling behaviour of hy drogels. Mol. Phys. 110, 1391-1402(2012).

14. Hirokawa, Y.\& Tanaka, T. Volume phase transition in a non-ionic gel. in 203, 203-208(1984).

15. Peng, H.-Y. et al. Smart Hydrogel Gratings for Sensitive, Facile, and Rapid Detection of Ethanol Concentration. Ind. Eng. Chem. Res. 58, 17833-17841(2019).

16. Ono, Y. \& Shikata, T. Hydration and Dynamic Behavior of Poly(Nisopropylacrylamide)s in Aqueous Solution: A Sharp Phase Transition at the Lower Critical Solution Temperature. J. Am. Chem. Soc. 128, 10030-10031(2006).

17. Okada, Y.\& Tanaka, F. Cooperative Hydration, Chain Collapse, and Flat LCST Behavior in Aqueous Poly(N-isopropylacrylamide) Solutions. Macromolecules 38, 4465-4471 (2005).

18. Philipp, M. et al. From Molecular Dehydration to Excess Volumes of Phase-Separating PNIPAM Solutions. J. Phys. Chem. B 118, 4253-4260 (2014).

19. Pang, J., Yang, H., Ma, J. \& Cheng, R. Solvation Behaviors of N Isopropylacrylamide in Water/Methanol Mixtures Revealed by Molecular Dynamics Simulations. J. Phys. Chem. B 114, 8652-8658(2010). 
20. Winnik, F. M., Ringsdorf, H. \& Venzmer, J. Methanol-water as a cononsolvent system for poly ( $\mathrm{N}$-isopropylacrylamide). Macromolecules 23,2415-2416(1990).

21. Schild, H. G., Muthukumar, M. \& Tirrell, D. A. Cononsolvency in mixed aqueous solutions of poly ( $\mathrm{N}$-isopropylacrylamide). Macromolecules 24, 948-952(1991).

22. Zhang, G. \& Wu, C. Reentrant Coil-to-Globule-to-Coil Transition of a Single Linear Homopolymer Chain in a W ater/Methanol Mixture. Phys. Rev. Lett. 86, 822-825(2001).

23. Zhang, G. \& Wu, C. The Water/Methanol Complexation Induced Reentrant Coil-to-Globule-to-Coil Transition of Individual Homopolymer Chains in Extremely Dilute Solution. J. Am. Chem. Soc. 123,1376-1380 (2001).

24. Tanaka, F., Koga, T. \&Winnik, F. M. Temperature-Responsive Polymers in Mixed Solvents: Competitive Hydrogen Bonds Cause Cononsolvency. Phys. Rev. Lett. 101, 028302 (2008).

25. Okada, Y.\& Tanaka, F. Cooperative hydration, chain collapse, and flat LCST behavior in aqueous poly(N-isopropylacrylamide) solutions. Macromolecules 38, 4465-4471 (2005).

26. Dudowicz, J., Freed, K. F. \& Douglas, J. F. Relation between Solvent Quality and Phase Behavior of Ternary Mixtures of Polymers and Two Solvents that Exhibit Cononsolvency. J. Phys. Chem. B (2016). doi:10.1021/acs.jpcb.6b03248

27. Tanaka, F., Koga, T., Kojima, H., Xue, N. \& Winnik, F. M. Preferential Adsorption and Co-nonsolvency of Thermoresponsive Polymers in Mixed Solvents of Water/Methanol. Macromolecules 44, 2978-2989 (2011).

28. Mukherji, D., Marques, C. M. \& Kremer, K. Polymer collapse in miscible good solvents is a generic phenomenon driven by preferential adsorption. Nat. Commun. 5, 1-6 (2014).

29. Zuo, T. et al. Water/Cosolvent Attraction Induced Phase Separation: A Molecular Picture of Cononsolvency. Macromolecules 52, 457-464 (2019).

30. Bischofberger, I., Calzolari, D. C. E., De Los Rios, P., Jelezarov, I. \& Trappe, V. Hydrophobic hydration of poly-N-isopropyl acrylamide: a matter of the mean energeticstate of $w$ ater. Sci. Rep. 4, 4377 (2015).

31. Tavagnacco, L., Zaccarelli, E. \& Chiessi, E. Molecular description of the coil-to-globule transition of Poly( $\mathrm{N}$-isopropylacrylamide) in water/ethanol mixture at low alcohol concentration. J. Mol. Liq. 297, 111928 (2020).

32. Liu, G. \& Zhang, G. Reentrant Behavior of Poly(N -isopropylacrylamide) 
Brushes in Water-Methanol Mixtures Investigated with a QuartzCrystal Microbalance. Langmuir 21, 2086-2090(2005).

33. Yu, Y. et al. Tunable friction by employment of co-non-solvency of PNIPAMbrushes. Polymer (Guildf). 102, 372-378 (2016).

34. Kreuzer, L. P. et al. Poly(sulfobetaine) versus Poly( N isopropylmethacrylamide): Co-Nonsolvency-Type Behavior of Thin Films in a Water/Methanol Atmosphere. Macromolecules acs.macromol.0c02281 (2021). doi:10.1021/acs.macromol.0c02281

35. Bisw as, C. S., Patel, V.K., Vishwakarma, N. K., Mishra, A. K. \& Ray, B. Synthesis and characterization of porous poly(N-isopropylacrylamide) hydrogels prepared in ethanol-water mixtures. J. Appl. Polym.Sci. 121, 2422-2429(2011).

36. Scherzinger, C., Schwarz, A., Bardow, A., Leonhard, K. \& Richtering, W. Cononsolvency of poly-N-isopropyl acrylamide (PNIPAM): Microgels versus linear chains and macrogels. Curr. Opin. Colloid Interface Sci. 19, 84-94(2014).

37. Lucas, R. Ueber das Zeitgesetz des kapillaren Aufstiegs von Flüssigkeiten. Kolloid-Zeitschrift 23, 15-22(1918).

38. Washburn, E. W. The Dynamics of Capillary Flow. Phys. Rev. 17, 273283 (1921).

39. Xia, Y. \& Whitesides, G. M. SOFT LITHOGRAPHY. Annu. Rev. Mater. Sci. 28, 153-184 (1998).

40. Ebara, M., Hoffman, J. M., Hoffman, A. S. \& Stayton, P. S. Switchable surface traps for injectable bead-based chromatography in PDMS microfluidic channels. Lab Chip 6, 843(2006).

41. Ballal, D. \& Chapman, W.G. Hydrophobic and hydrophilic interactions in aqueous mixtures of alcohols at a hydrophobic surface. J. Chem. Phys. 139, 114706 (2013).

42. Bischofberger, I., Calzolari, D. C. E. \& Trappe, V. Co-nonsolvency of PNiPAM at the transition between solvation mechanisms. Soft Matter 10, 8288-8295(2014).

43. Nishi, N. et al. Hydrogen-Bonded Cluster Formation and Hydrophobic Solute Association in Aqueous Solutions of Ethanol. J. Phys. Chem. 99, 462-468(1995).

44. Dong, Q., Qian, M. \& Luo, C. Phase separation behavior and fluorescence properties of poly(N-isopropylacrylamide) $\mathrm{w}$ ith chalcone side groups in mixed solvents. J. Polym. Res. 23, 33 (2016).

45. Zhang, Y., Furyk, S., Bergbreiter, D. E. \& Cremer, P.S. Specific ion effects on the w ater solubility of macromolecules: PNIPAM and the Hofmeister series. J. Am. Chem. Soc. (2005). 
Chapter 4 


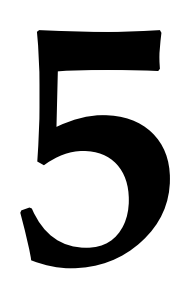

Integration of Air-pockets and Pillared Stop Valves in Microchannel for Capillary Flow Control 


\begin{abstract}
utonomous capillary systems where a liquid is driven by capillarity are

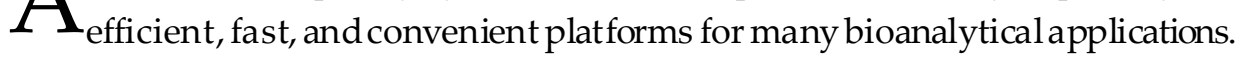
However, accurate control of the flow of liquids in capillarity-driven microfluidics is not easy due toits spontaneous nature. In this chapter, we report on a capillarity-driven microfluidic device with integrated thermo-sensitive hydrophobicstop valves and air gates to manipulate the liquid flow. The control unit consists of a microchannel with one partially pillared bottom $w$ all and two air pockets connected to its sidewalls. The channel surface w as coated with a thermos-sensitive polymer, PNIPAm. By actuating the temperature in the range of $20-40^{\circ} \mathrm{C}$, either the hydrophobic PNIPAm surface or the air expansion in the pockets generating an air gate will stop the liquid flow. By integrating multiple working units in a microfluidic device, the liquid flow path becomes programmable.
\end{abstract}

tThis chapter is based on the article manuscript: L. Li, L. Shui, M. Odijk, and J.C.T. Eijkel. Integration of air-pockets and pillared stopvalve in a microchannel for capillary flow control. In preparation 


\subsection{INTRODUCTION}

Capillary microfluidics, also called "passive" microfluidics, is a type of microfluidics using capillary action to manipulate liquids in capillaries or microchannels without the requirement of external pum ping mechanisms. ${ }^{1}$ It has been associated with several advantages such as being self-powered, having small sample volume requirements, cost-effective fabrication, easy operation, and portability. ${ }^{2}$ Taking advantage of these properties, capillary microfluidics has been widely used for immunoassays and diagnostic applications..$^{3-5}$ For those applications, accurate sample volumes to reach high sensitivity and controllable flow rate to improve diagnostic assay performance become im portant. ${ }^{6}$

Capillary action is governed by the surface tension of a liquid and its adhesion to a solid surface. In microchannel-based capillary systems, the filling behavior of a liquid is dependent on the w ettability of the channel $w$ alls and the channel geometry. ${ }^{7}$ Autonomous filling occurs in microchannels with a hydrophilic surface. By control the surface wettability of the device and the channel geometry, the liquid can be delivered in a programmed way. In capillary channels of constant cross-section, the liquid behavior generally follows the well-known Washburn behavior, characterized by a flow rate that decreases $w$ ith the square root of time. By varying geometric parameters, e.g. narrowing/expanding a single channel, ${ }^{8}$ integrating microstructure arrays with different shapes, density and relative positions,, 10 serial connection of capillary pumps, or locally creating a higher capillary pressure by a narrower geometry, ${ }^{11,12}$ the capillary flow rate can be increased, decreased or even be kept nearly constant. Recently, new findings on a constant capillary flow rate in a Poly( $\mathrm{N}$-isopropylacrylamide) (PNIPAm) hydrogel-coated capillary due to "stick-slip" motion have been reported, 13 broadening the knowledge base for controlling capillary flow in microchannels. In chapter 4, we reported on a PNIPAm-grafted PDMS (PNIPAm-g-PDMS) microchannel where a constant filling flow rate $w$ as also observed.

Capillary flow can be stopped by creating hydrophobic patches ${ }^{14,15}$ on the channel walls or by abruptly enlarging the channel diameter ${ }^{16-18}$ without external equipment. Alternative methods such as using a temperature-responsive polymer on the surface and delivering a temperature stimulus ${ }^{15,19}$ or using the electro-wetting phenomenon ${ }^{20,21}$ have also been reported to create stopvalves to control capillary flow. How ever, these valves are not as flexible as active valves such as pneumatic valves since they only have a stop function at the liquid front. 
Once the liquid passes the valving area, they won't be able to discontinue it. Complex programming of flow in a liquid circuit thus becomes difficult in capillary microfluidic devices, increasing the interest in integrating active valves in passive flow systems for liquid manipulation.

Bubbles have become a new control unit in microfluidics since they are simple to gener ate and the bubbles are compressible and bio-compatible. Air bubbles have been widely used as micropumps, ${ }^{22,23}$ microvalves, ${ }^{24,25}$ for micromixing, ${ }^{26}$ for liquid manipulation, ${ }^{24,27}$ and concentration gradient generation. ${ }^{28}$ In capillary microfluidic devices, both thermal expansion ${ }^{25}$ and electrochemical bubble generation ${ }^{29}$ are commonly used for air bubble generation.

In chapter 3, we presented a PDMS microfluidic device coated with the thermosensitive polymer PNIPAm enabling temperature-triggered stop valve function. The device exhibits a constant capillary filling speed which would be beneficial for example for immunoassays to have a steady flow during the incubation step. How ever, the stop valve doesn't work in freshly coated channels since the hydrophobicity of a smooth PNIPAm-g-PDMS surface is not sufficient to stop the flow. In this chapter, we solved this problem by machining a pillar array on the surface, where the pillared PNIPAm-g-PDMS surface exhibits a contact angle of $127^{\circ}$ at $40{ }^{\circ} \mathrm{C}$, and stop valve function could be obtained in a freshly coated PNIPAm-g-PDMS channel. Moreover, we integrated air pockets aside from the hydrophobic pillared patch, where an air gate can be easily formed in $1.15 \mathrm{~s}$ by thermal expansion of air by a temperature increase from 20 to $36{ }^{\circ} \mathrm{C}$. The integration of pillared PNIPAm-g-PDMS stop valves and the air gates were subsequently used for programming liquid delivery in microfluidic devices. Figure 5.1 shows the schematic overview of the valving units controlling the flow path in the device. 


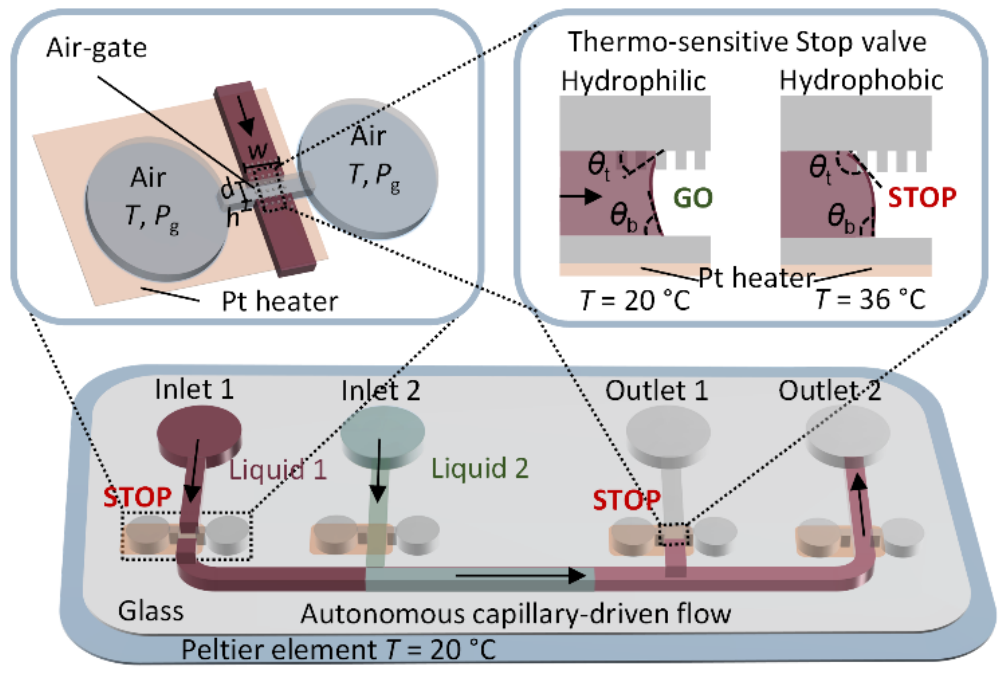

Figure 5.1 Schematic overview of a capillarity-driven microfluidic device with integrated air gates and thermo-sensitive hydrophobic stop valves for liquid manipulation. The liquid is autonomously transported in the hydrophilic microchannel by capillary action. The flow will be stopped by a thermo-sensitive stop valve due to meniscus pinning at the liquid front at $36^{\circ} \mathrm{C}$. The flow will resume when the temperature is lowered to $20^{\circ} \mathrm{C}$. An air gate can again pause the liquid flow due to air expansion from closed air pockets with increasing temperature. The integration of two valves can control the delivery of two liquids to the main channel and deliver a mixture of two liquids or a single liquid to a specific outlet. Platinum (Pt) heaters fabricated in the bottom glass wafer and a Peltierelement with a constant temperature of $20^{\circ} \mathrm{C}$ are used to locally control the temperature of the device.

\subsection{MATERIALS AND METHODS}

\subsubsection{Materials}

In this chapter, most of the materials used are the same as in chapter 3 . Food dyes (Jo-La, Surinam) were used for liquid coloring.

\subsubsection{PDMS device fabrication}

The polydimethylsiloxane (PDMS) microfluidic devices were fabricated using standard photolithography techniques ${ }^{30}$. Silicon wafers with patterned SU-8 structures were used as molds. Hole arrays with controlled dimensions were created on SU-8 to obtain pillared PDMS on the entire PDMS surface or locally on one of the PDMS microchannel walls. The mask design for mold fabrication is presented in Figure D1 and D2 in Appendix D. The detailed fabrication 
process of the SU-8 molds and PDMS chips is presented in Figure D3 in Appendix D. The photographs of the PDMS chip are shown in Figure D4 in Appendix D.

\subsubsection{Chip coating with PNIPAm}

The chip was coated with PNIPAm by UV-initiated surface polymerization. ${ }^{31}$ The detailed grafting method was presented in chapter 3. A shadow mask was used to obtain local grafting of PNIPAm inside the PDMS microchannel to ensure the surface of the air pockets remains hydrophobic.

\subsubsection{Contact angle mea surements}

The dynamic wetting of water droplets on the PNIPAm-g-PDMS pillared substrates was investigated by dropping a single drop of $w$ ater with a volume of $10 \mu \mathrm{L}$ and measured with an interfacial tension meter OCA 15 Pro (Dataphysics, Germany). The advancing/receding contact angle w as obtained by continuously increasing/decreasing the volume of the droplet from $2 \mu \mathrm{L}$ to $12 \mu \mathrm{L}$ at a speed of $2 \mu \mathrm{L} / \mathrm{s}$.

\subsubsection{Heater and sensor fabrication}

The detailed fabrication process $\mathrm{w}$ as presented in chapter 3 . The schematic design of the microheaters and sensors can be found in Figure D5 in Appendix D.

\subsubsection{Temperature sensor calibration}

The calibration method was presented in chapter 3 ..

\subsection{RESULTS ANDDISCUSSION}

\subsubsection{Contact angle (CA) of w ater on PNIPAm-g-PDMS pillared surface}

Bare PDMS exhibits a hydrophobic nature with a water CA of $105^{\circ}$. With micro/nano-arrays fabricated on its surface, we can obtain super-hydrophobic surfaces with CAs greater than $150^{\circ} .{ }^{32}$ By grafting the surface with PNIPAm, a thermo-sensitive hydrophilic/hydrophobic surface can be obtained. The static CAs on a smooth PNIPAm-g-PDMS surface ranges from $~ 70$ to 103 in the temperature range of $20-40^{\circ} \mathrm{C}$ (according to Figure 3.4 in chapter 3 ) On a rough solid surface, e.g. a micro/nano-structure patterned surface, the wettability is frequently represented by the apparent $\mathrm{CA}\left(\theta_{\mathrm{AP}}\right)$ of a liquid droplet on the surface. 
The Wenzel ${ }^{33}$ (surface is wetted by the droplet) and Cassie-Baxter ${ }^{34}$ (droplet is seated only on top of the rough features of the surface) models are used to describe the w etting state on a rough surface.

To study the CAs on pillared PNIPAm-g-PDMS surfaces, a square array of cylindrical PDMS micropillars on PDMS $w$ as fabricated. Then w e coated the surface with PNIPAm and measured the dynamic water CAs, including static apparent $\mathrm{CA}\left(\theta_{\mathrm{s}}\right)$, advancing $\mathrm{CA}\left(\theta_{\mathrm{a}}\right)$ and receding $\mathrm{CA}\left(\theta_{\mathrm{r}}\right)$ on both pillared PDMS surfaces and pillared PNIPAm-g-PDMS surfaces. Results are shown in Figure 5.2.

(a) Pillared PDMS surface

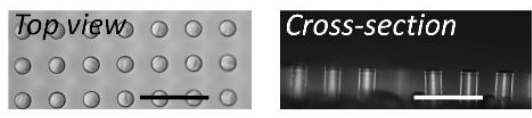

(b) CAs on pillared PDMS surface

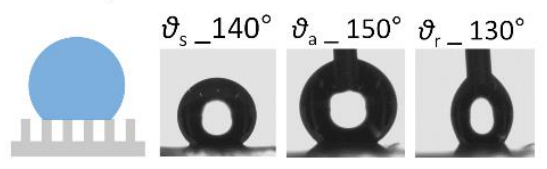

(c) CAs on pillared PNIPAm-g-PDMS surface

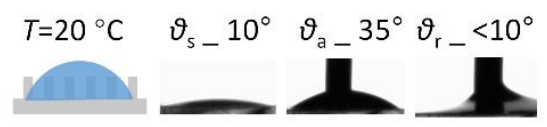

(d) CAs on pillared PNIPAm-g-PDMS surface

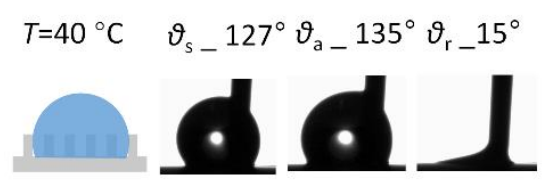

Figure 5.2 CA of water on pillared PDMS surface and a pillared PNIPAm-g-PDMS surface. (a) Microscope images of a square array of cylindrical PDMS micropillars. The PDMS pillars are $20 \mu \mathrm{m}$ high. The diameter of the micropillars is $10 \mu \mathrm{m}$ and the center-to-center distance is $25 \mu \mathrm{m}$. (b) CAs on a pillared PDMS surface (Cassie state). (c) CAs on a hydrophilic pillared PNIPAm-g-PDMS surface at $20{ }^{\circ} \mathrm{C}$. (d) CAs on a hydrophobic pillared PDMS surface at $40^{\circ} \mathrm{C}$ (Wenzel state).

CAs on PDMS surfaces with the dimensions given in Figure 5.2 exhibit CassieBaxter state with $\theta_{\mathrm{s}}, \theta_{\mathrm{a}}, \theta_{\mathrm{r}}$ of $140^{\circ}, 150^{\circ}$ and $130^{\circ}$, respectively. After coating with PNIPAm, the pillared surface become very hydrophilic at $20^{\circ} \mathrm{C}$. The water droplet fills the gap between the micropillars, and a small $\theta_{\mathrm{s}}, \theta_{\mathrm{a}}$, and $\theta_{\mathrm{r}}$ of $10^{\circ}, 35^{\circ}$ and $\mathrm{CA}<10^{\circ} \mathrm{w}$ as observed. When the temperature of the pillared PNIPAm-gPDMS surface is $40^{\circ} \mathrm{C}$, the surface becomes hydrophobic with a $\theta_{\mathrm{s}}, \theta_{\mathrm{a}}$ of $127^{\circ}$, 
$135^{\circ}$, respectively. The wetting at $40^{\circ} \mathrm{C}$ is in the Wenzel state since we observed that the water $w$ as filling into the gaps between pillars. As a result, the system show s a small receding angle of $15^{\circ}$, indicating the pinning of $w$ ater inside the micropillars. Compared with the water CA changes on smooth PNIPAm-gPDMS surface, the pillared surface shows a wider range in CA change with temperature.

5.3.2 Dimensional and temperature effects on the wetting behavior of water on PNIPAm-g-PDMS pillared surface

The w etting behavior of a liquid droplet on a surface is affected by surface energy and surface topography. ${ }^{35}$ In this case the dimension of the micropillars plays an important role in controlling the CAs of the pillared PNIPAm-g-PDMS surface. Figure 5.3a shows a schematic drawing of a surface $w$ ith a pillared array with a pillar diameter of $a$, pillar distance of $d$, and pillar height of $H$. On a pillared PNIPAm-g-PDMS surface with fixed values of $a$ and $b$, the apparent CA will increase with $H$ as the surface is hydrophobic and the roughness ratio increases with $H$, since the water on the surface is in the Wenzelstate. ${ }^{36}$

Figure 5.3b shows the time-dependent $\mathrm{CA}$ on pillared PNIPAm-g-PDMS surfaces with pillar height $H$ of $5 \mu \mathrm{m}, 10 \mu \mathrm{m}$, and $20 \mu \mathrm{m}$ at 20 and $40^{\circ} \mathrm{C}$. At $20^{\circ} \mathrm{C}$, the surface is hydrophilic, and with increasing $H$, we see an increase in advancing $\mathrm{CA}$ and a decrease in static $\mathrm{CA}$. The contact angle decreases with time due to the intrusion of $w$ ater into the gaps between the micropillars. At $40{ }^{\circ} \mathrm{C}$, where the PNIPAm collapses on the PDMS surface and the surface becomes hydrophobic due to the absence of PNIPAm hydration, no time-dependent wetting was observed. The static $C A$ increases with increasing $H$ due to the increase in surface roughness. The surface with $20 \mu \mathrm{m}$ high pillars shows the highest water CA at $40^{\circ} \mathrm{C}$. A better stop valve function can thus be obtained using a surface with 20 $\mu \mathrm{m}$ high pillars. 
(a) Pillared surface
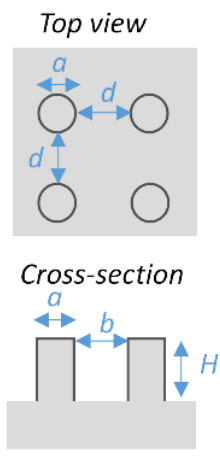

(b)

CAs on pillared PNIPAm-g-PDMS surface

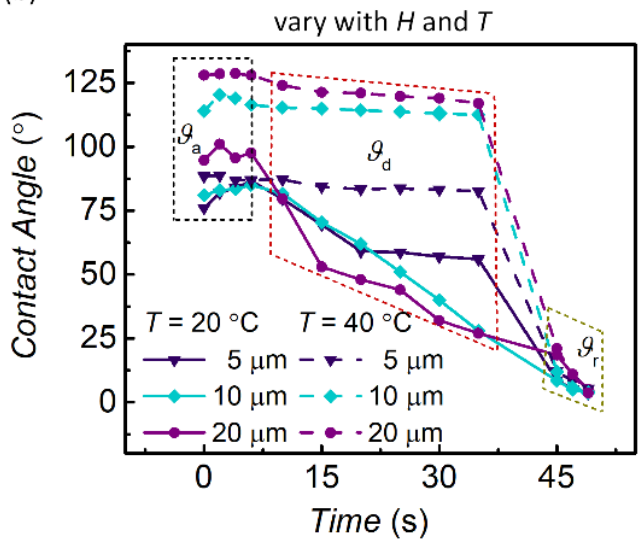

Figure 5.3 Pillar height and temperature effects on the wetting behavior of water on a PNIPAm-g-PDMS pillared surface. (a) Schematic drawing of a surface with a pillar array. The pillar diameter is $a$, pillar to pillar distance is $d$ and pillar height is $H$. (b) Timedependent CAs on a pillared PNIPAm-g-PDMS surface at $20^{\circ} \mathrm{C}$ and $40^{\circ} \mathrm{C}$. The PNIPAmg-PDMS pillars are 5, 10, $20 \mu \mathrm{m}$ high. $\theta_{\mathrm{a}}, \theta_{\mathrm{d}}, \theta_{\mathrm{r}}$ are advancing CA, dynamic CA and receding CA on the surface, respectively.

5.3.3 Temperature-controlled stop valve with pillared PNIPAm-g-PDMS for capillarity-driven flow control

In chapter 3, the thermo-sensitive wettability of PNIPAm-g-PDMS was used to create a stop valve to control capillarity-driven flow. The freshly coated surface didn't allow temperature control since its hydrophobicity at higher temperatures w as not large enough to stop the capillary flow in the microchannel. In this chapter, we show that a surface with $20 \mu \mathrm{m}$ high pillars which shows a higher $\mathrm{CA}$ at $40^{\circ} \mathrm{C}$, makes it possible to obtain stop valving in the freshly coated channels.

To study the thermally controlled stop valving function in a pillared PNIPAm-gPDMS channel, we made a PDMS microchannel consisting of a bottom wall fully machined with PDMS pillar arrays and the top, left, right walls with a smooth PDMS surface. Pillar ar rays with $a=10 \mu \mathrm{m}, d=15 \mu \mathrm{m}$, and $H=20 \mu \mathrm{m}$ were designed and fabricated on the bottom channel wall. The height $(h)$ of the channel is $60 \mu \mathrm{m}$. The channel $w$ as coated $w$ ith PNIPAm using the method described in 3.3.5 in chapter 3 . 
(a)

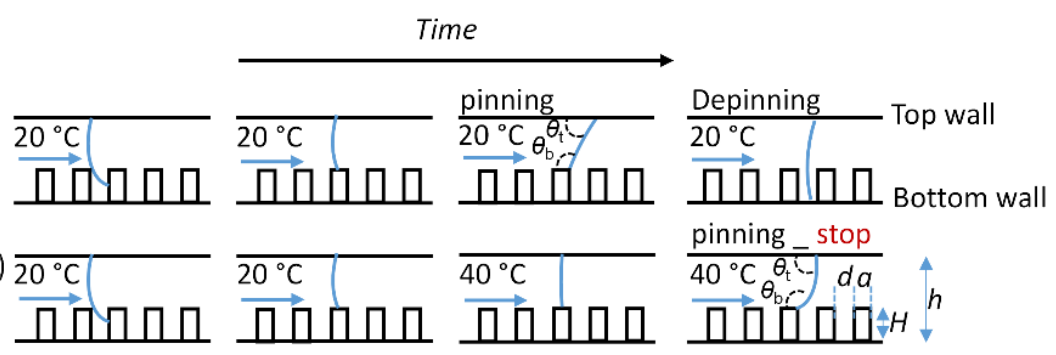

(b)

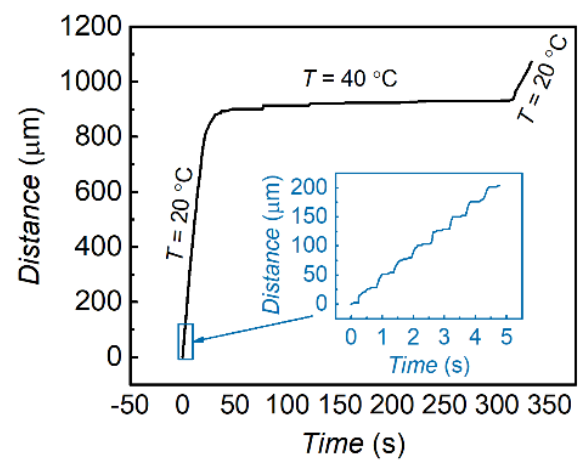

Figure 5.4 Temperature-controlled stop valving in the pillared PNIPAm-g-PDMS microchannel. (a) Schematic drawing of the centerline meniscus profile and contact angle change during liquid filling at 20 (i) and $40{ }^{\circ} \mathrm{C}$ (ii). (b) Centerline contact line position measured as a function of time in response to temperature.

Figure 5.4a schematically shows the meniscus profile at the center of the channel determined by the liquid contact line position and contact angle on the channel walls. At $20^{\circ} \mathrm{C}$, the static water CA on a smooth PNIPAm-g-PDMS surface is $70^{\circ}$, thus the contact angle on the top channel wall $\theta_{\mathrm{t}}$ is $\sim 70^{\circ}$, the same as the contact angle on the side walls (in our device, we assume equal contact angles on top and sidew alls as discussed in chapter 3, 3.4.2). When the advancing fluid reaches the beginning of a pillar, liquid im pinging on the pillar surface will have a CA near $90^{\circ}$, resulting in deformation of the liquid front until it reaches the top front of the pillar. When the liquid front reaches the end of the top pillar, pinning will occur at the edge of the pillar top causing an increase in contact angle $\left(\theta_{\mathrm{b}}\right)$. During the pinning, liquid on the bottom keeps moving since the CA on the side w alls as w ell as the pillar surface remains $70^{\circ}$. Depinning occurs when the liquid moves to the other side of the pillar, resulting in an acceleration of the liquid front ${ }^{3740}$ Figure $5.4 \mathrm{~b}$ shows the deceleration and acceleration of the contact line at $20^{\circ} \mathrm{C}$ (blue line graph). At $40{ }^{\circ} \mathrm{C}$, the PNIPAm-g-PDMS surface becomes hydrophobic w ith a static $\mathrm{CA} \theta_{t}$ near $90^{\circ}$. When the liquid front now reaches the 
edge of the pillar top, the increase of $\theta_{b}\left(\theta_{b}>90^{\circ}\right)$ and hydrophobic PNIPAm-gPDMS surface contribute to a positive Laplace pressure which results in a convex meniscus (Figure 5.4 a(ii)) at the liquid front, causing the liquid to stop (Figure $5.4 \mathrm{~b})$. Restarting of the liquid can be affected by decreasing the temperature to $20{ }^{\circ} \mathrm{C}$ (Figure 5.4b). A video of the temperature-controlled stop valving in PNIPAM-g-PDMS microchannel with integrated micropillars was added to the Appendix D5 (video 1).

5.3.4 Air gate induced by thermal expansion of gas in a closed air pocket as a stop valve.

The stop valve with the temperature-responsive hydrophilic/hydrophobic surface w orks well for controlling liquid movement at the air/liquid interface. However, once the surface is wetted by water, the stop valve will lose its functionality, which makes it difficult to program liquid circuits. The problem can be easily solved by integrating air pockets to generate an air gate within the channel, as reported by van der Wijngaart et.al...11 We designed and fabricated tw o air pockets connected to the main channel aside from the hydrophobic patch.

Figure 5.5a shows the schematic drawing of the w orking unit. The channel area w as grafted with PNIPAm while the surface inside the air pockets is hydrophobic (surface modification methods in section 5.2.3). The air-pockets are connected to the channel by an open window with height $h$ and width $d$. The air expansion induced by increasing the temperature in the air pocket results in an air gate connecting the two air pockets and discontinues the capillary filling of liquid.

Figure $5.5 \mathrm{~b}$ shows the cross-section of the air gate.

(a)

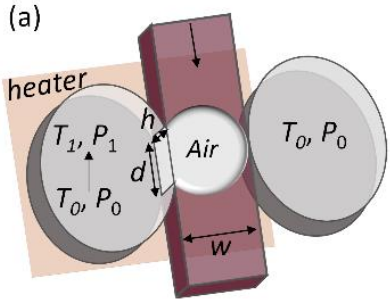

(b)

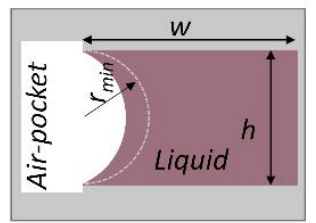

(c) $t=0 \mathrm{~s}$

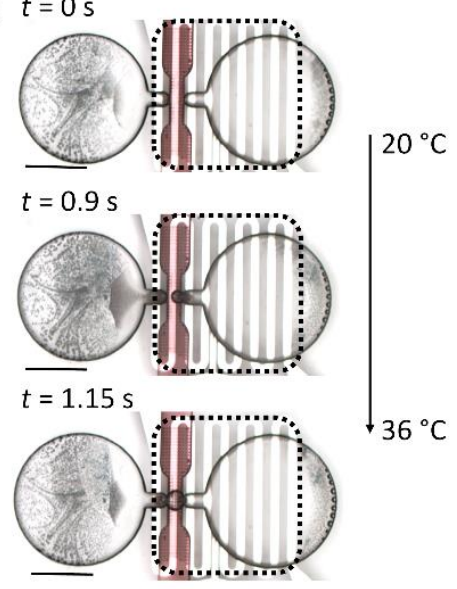


Figure 5.5 Air gate induced by thermal expansion of gas in a closed air pocket as microvalve. (a) Schematic drawing of the air bubble formation in the microchannel. The air pocket is connected with the channel through an open window with width $d$ and height $h$. The channel width is $w$. A heater was placed underneath the air pocket. With an air temperature increase from $T_{0}$ to $T_{1}$, the air pressure in the pocketincreases from $P_{0}$ to $P_{1}$. (b) Cross-section of the air gate. The dashed line indicates the interface where the gas bubble radius reaches its minimum $\left(r_{\min }\right)$. (c) Photomicrographs of air gate formation in the channel by a temperature increase from $20^{\circ} \mathrm{C}$ to $36^{\circ} \mathrm{C}$. Dashed squares indicate the heating area. Scale bar denotes $500 \mu \mathrm{m}$.

The theoretical analysis of the functioning of the air valve can be found in reference 41 . We will approximately follow it here. The pressure difference between the gas in the bubble and the liquid, $\Delta P$, (with the pressure in the gas being higher) is given by the Young-Laplace equation

$$
\Delta P=\gamma\left(\frac{1}{r_{1}}+\frac{1}{r_{2}}\right)
$$

Here $\gamma$ is the surface tension of the liquid and $r_{1}$ and $r_{2}$ are the principal radii of curvature of the bubble.

In our device, we design the channel and air pockets with $d \approx h<w$. On a temperature increase, the pressure in the bubble will increase, as well as its volume. The expanding gas bubble will suddenly intrude into the main channel once its radius passes the geometrical minimum $\left(r_{\min }\right) .{ }^{41}$ For the geometry in Figure $5.5 \mathrm{~b}, r_{\min } \approx h / 2 \approx d / 2$, so that equation (1) for the burst pressure by approximation is

$$
\Delta P_{\text {burst }}=\frac{4 \gamma}{h}
$$

$\triangle P_{\text {burst }}$ is the burst pressure to form an air gate in the channel. Since the volume of the air pocket is much larger than the water displacement $\Delta V$, the gas bubble volume is approximately constant so that the pressure in the air pocket at the moment of bursting can be estimated from the law of Gay-Lussac,

$$
P_{0} T_{1}=P_{1} T_{0}
$$

Here $P_{1}$ and $P_{0}$ are the pressure in the air pocket at temperature $T_{1}$ and $T_{0}\left(T_{1}>\right.$ $\left.T_{0}\right)$, respectively.

The increase in temperature needed to reach the burst pressure therefore is 100 


$$
T_{1}-T_{0}=T_{0} \frac{\Delta P_{\text {burst }}}{P_{1}}=T_{0} \frac{4 \gamma}{h P_{0}}
$$

In our system, $T_{0}=293 \mathrm{~K}, P_{0}=10^{5} \mathrm{~Pa}$, and the channel is $45 \mu \mathrm{m}$ high. According to equation 4 , the air gate $w$ ill then be generated by an estimated temperature increase of $18 \mathrm{~K}$. In our experiment, we found that the air gate could be generated in $1.15 \mathrm{~s}$ by increasing the temperature from $20^{\circ} \mathrm{C}$ to $36^{\circ} \mathrm{C}$ (Figure 5.5c), which is $2^{\circ} \mathrm{C}$ low er than the calculated temperature increase. That the gate closes at a slightly lower temperature may be due to the contribution of the $w$ ater vapor pressure, as we indeed see water condensation in the colder area. The increase of partial water vapor pressure between $20^{\circ} \mathrm{C}$ and $36^{\circ} \mathrm{C}$ is about is about $2.7 \mathrm{kPa}$, which could provide the needed extra pressure. In our system, since air can't replace water on a wetted PNIPAm surface, ${ }^{42}$ we integrated two air pockets on each side of the channel to make sure the air gate can completely prevent the liquid flow at the higher temperature.

In our experiment, we found that the air gate w on't open anymore after several w orking cycles (video 2 in Appendix D presents a valving failure after 4 working cycles), which may be due to the water evaporation and condensation that permanently forms an air plug in the channel. Future work is suggested to connect the unheated air pocket to the atmosphere to form an air vent to avoid a permanent air plug. By doing so, the air gate can still be formed at $36^{\circ} \mathrm{C}$ by thermal expansion of air in the heated air pocket. When the two air pockets are connected, the air pressure will decrease, however, the liquid will stop since the pillared PNIPAm-g-PDMS surface has become hydrophobic. At a subsequent temperature decrease, the valving area becomes an open area again and the formation of a permanent air plug will be avoided. The liquid filling will thus continue when decreasing the temperature.

5.3.5 Integration of stop valves and air gates for capillary flow control in a microchannel.

The stop valving function of the pillared PNIPAm-g-PDMS surface can be used to control sample loading and guiding of the liquid front, and the air gate function can be used to discontinue the liquid flow once the liquid has passed the PNIPAm-g-PDMS stop valve. The integration of these tw o working units in microfluidic devices can be utilized for programmed liquid circuit flow. 
(a) Hydrophobic stop valves control sample loading

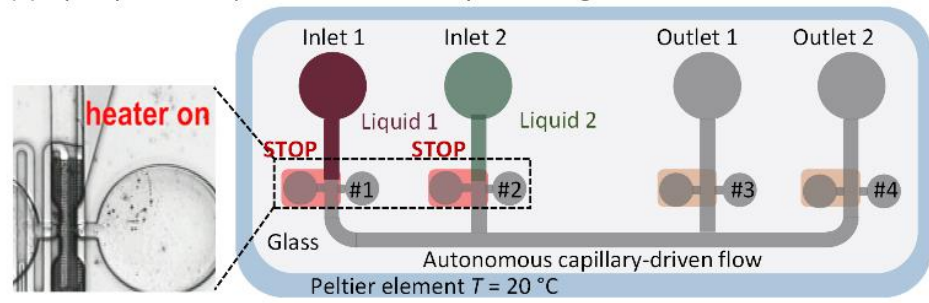

(b) Heater off allows sample filling and mixing; Stop valve allows liquid routing

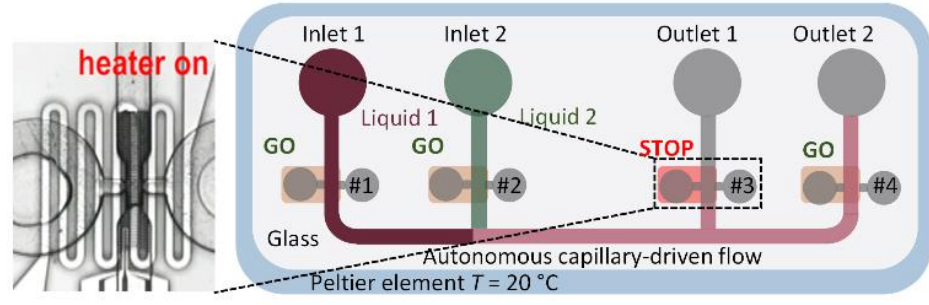

(c) Air gate 1 closed (heater 1 on)_ liquid 1 stops filling

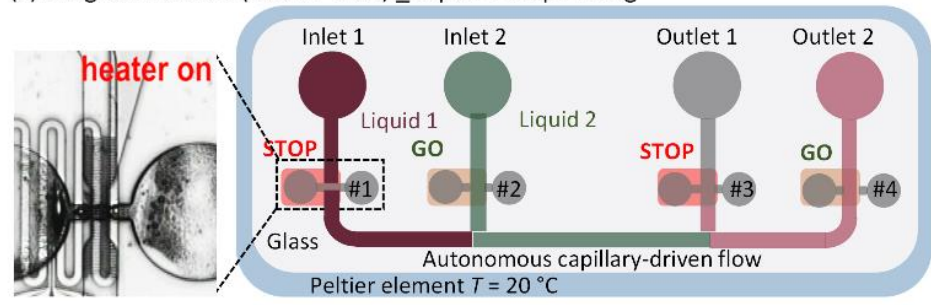

(d) Heater off guides liquid 2 to outlet 1

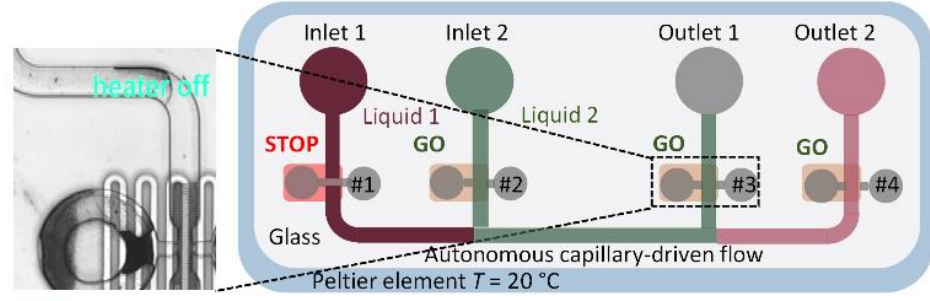

$$
\text { Heater on }-T=36{ }^{\circ} \mathrm{C} \quad \text { Heater off } \_T=20^{\circ} \mathrm{C}
$$

Figure 5.6 Controllable sequential liquid delivery using integrated pillared PNIPAm-g PDMS stop valves and air pockets. $\# 1, \# 2, \# 3, \# 4$ designate the four working units with integ rated air pockets and stop valve. (a) Stopvalve control of sample loading. Heater 1 and 2 on, liquids 1 and 2 stopped by the stop valves. (b) Liquid mixing and liquid routing. Heater 1 and 2 off, stop valves 1 and 2 closed, liquid 1 and 2 are released and mix in the main channel. Heater 3 on, stop valve 3 open, liquid stopped at valve 3, the mixture delivered to outlet 2. (c) Air gate discontinuing of liquid filling. Heater 1 on, air gate 1 closed, liquid 1 stopped by the air gate, liquid 2 delivered to the main channel. (d) Heater 
3 off, guiding liquid 2 to outlet 1 . Here the liquid stop/discontinued when the stop valve open and air gate close.

Figure 5.6 shows liquid mixing and specificliquid delivery using the stop valve and air gate combination. Liquid 1 and liquid 2 were loaded from inlet 1 and inlet 2 respectively to their specific channel by capillary action and stopped before the pillared area (we call this area 'loading point') by the pillared PNIPAm-g-PDMS stop valve (Figure 5.6a). Then the liquid was released by turning off the heaters 1 and 2 at the loading points, releasing liquid 1 and 2, and allowing liquid mixing in the main channel. The liquid mixture could then be delivered specifically to outlet 2 by closing stop valve 3 (Figure 5.6b). Subsequently, the delivery of liquid $1 \mathrm{w}$ as stopped by closing air gate 1 , and liquid 2 started filling the main channel (Figure 5.6c). By opening stop valve 3, liquid $2 \mathrm{w}$ as then delivered to outlet 1(Figure 5.6d). (A QR code of the whole videois presented in Appendix D Video 3)

\subsection{CONCLUSION AND OUTLOOK}

In this chapter, we reported the development of a capillary microfluidic device with integrated pillared PNIPAm-g-PDMS stop valves and air gates for programmable liquid delivery. The liquid fills the channel automatically due to the hydrophilic PNIPAm-g-PDMS surface at $20^{\circ} \mathrm{C}$. The pillared PNIPAm-gPDMS surface exhibits a contact angle of $127^{\circ}$ at $40^{\circ} \mathrm{C}$, w orking as a stop valve at the liquid front to control liquid loading and routing. The air pockets can form an air gate to discontinue liquid filling when the pocket temperature increases from 20 to $36^{\circ} \mathrm{C}$, due to the thermal expansion of the gas in the pocket. The integration of stop valves and air gates was used to control sample loading, mixing, and specific routing. The actuation temperature in these devices is compatible with bio-assays, and the device is easy to fabricate. With the constant filling flow rate in PNIPAm-g-PDMS channels and the double valving function, the device would be beneficial for precise, electrically actuated handling of liquids. Future w ork is suggested to integrate air venting by connecting one of the air pockets to the atmosphere to avoid a permanent bubble plug in the channel. High throughput manufacturing is recommended for handling more samples and perform multiple assays in a single chip.

\subsection{REFERENCES}

1. Zimmermann, M., Schmid, H.,Hunziker, P. \& Delamarche, E. Capillary pumps for autonomous capillary systems. Lab Chip (2007). 
2. Olanrewaju, A., Beaugrand, M., Yafia, M. \& Juncker, D. Capillary microfluidics in microchannels: From microfluidic networks to capillaric circuits. Lab Chip 18, 2323-2347 (2018).

3. Gervais, L., de Rooij, N. \& Delamarche, E. Microfluidic Chips for Pointof-Care Immunodiagnostics. Adv. Mater. 23, H151-H176 (2011).

4. Zimmermann, M., Hunziker, P. \& Delamarche, E. Autonomous capillary system for one-stepimmunoassays. Biomed. Microdevices 11, 1-8(2009).

5. Eicher, D. \& Merten, C. A. Microfluidic devices for diagnostic applications. Expert Rev. Mol. Diagn. 11,505-519(2011).

6. Wild, D. \& Kodak, E. The Immunoassay Handbook. The Immunoassay Handbook (Elsevier, 2013). doi:10.1016/C2010-0-66244-4

7. Delamarche, E., Juncker, D. \& Schmid, H. Microfluidics for Processing Surfaces and MiniaturizingBiological Assays. Adv. Mater. 17, 2911-2933 (2005).

8. van der Wijngaart, W. Capillary pumps with constant flow rate. Microfluid. Nanofluidics 16, 829-837 (2014).

9. Zimmermann, M., Schmid, H., Hunziker, P. \& Delamarche, E. Capillary pumps for autonomous capillary systems. Lab Chip 7, 119-125(2007).

10. Safavieh, R., Tamayol, A. \& Juncker, D. Serpentine and leading-edge capillary pumps for microfluidic capillary systems. Microfluid. Nanofluidics 18,357-366 (2015).

11. Juncker, D. et al. Autonomous Microfluidic Capillary System. Anal. Chem. 74, 6139-6144 (2002).

12. Guo, W., Hansson, J. \& van der Wijngaart, W. Capillary pumping independent of the liquid surface energy and viscosity. Microsystems Nanoeng. 4, 2 (2018).

13. Silva, J.E. et al.Stick-slip water penetration into capillaries coated with swelling hydrogel. Soft Matter 11,5933-5939 (2015).

14. Suk, J. W. \& Cho, J.-H. Capillary flow control using hydrophobic patterns. J. Micromechanics Microengineering 17, N11-N15 (2007).

15. Chunder, A., Etcheverry, K., Londe, G., Cho, H. J. \& Zhai, L. Conformal sw itchable superhydrophobic/hydrophilic surfaces for microscale flow control. Colloids Surfaces A Physicochem. Eng. Asp. 333, 187-193 (2009).

16. Eijkel, J. C. T. \& Van Den Berg, A. Young 4ever - The use of capillarity for passive flow handling in lab on a chip devices. Lab Chip 6, 1405-1408 (2006).

17. Glière, A. \& Delattre, C. Modeling and fabrication of capillary stop valves for planar microfluidic systems. Sensors Actuators, A Phys. (2006). doi:10.1016/j.sna.2005.12.011 
18. Papadimitriou, V. A., Segerink, L. I., van den Berg, A. \& Eijkel, J. C. T. 3Dcapillary stop valves for versatile patterning inside microfluidic chips. Anal.Chim. Acta (2018). doi:10.1016/j.aca.2017.11.055

19. Londe, G., Chunder, A., Wesser, A., Zhai, L. \& Cho, H. Microfluidic valves based on superhydrophobic nanostructures and switchable thermosensitive surface for lab-on-a-chip (LOC) systems. Sensors Actuators B Chem. 132, 431-438 (2008).

20. Satoh, W., Yokomaku, H., Hosono, H., Ohnishi, N. \& Suzuki, H. Electrowetting-based valve for the control of the capillary flow. J. Appl. Phys. 103, 034903(2008).

21. Arango, Y., Temiz, Y., Gökçe, O. \& Delamarche, E. Electro-actuated valves and self-vented channels enable programmable flow control and monitoring in capillary-driven microfluidics. Sci. Adv. 6, 1-13(2020).

22. Dijkink, R. \& Ohl, C. D. Laser-induced cavitation based micropump. Lab Chip (2008). doi:10.1039/b806912c

23. Chiu, S.-H. \& Liu, C.-H. An air-bubble-actuated micropump for on-chip blood transportation. Lab Chip 9, 1524(2009).

24. Prakash, M. \& Gershenfeld, N. Microfluidic bubble logic. Science (80-. ). 315, 832-835(2007).

25. Barman, U., Fiorini, P., Lagae, L. \& Jones, B. Capillary Microvalve Actuation Using Thermal Expansion of Trapped Air Bubble. Proceedings 2, 1031 (2018).

26. Hellman, A. N. et al. Laser-induced mixing in microfluidic channels. Anal.Chem. (2007). doi:10.1021/ac070081i

27. Khoshmanesh, K. et al. A multi-functional bubble-based microfluidic system. Sci. Rep.5, 9942 (2015).

28. Xu, Y., Lv, Y., W ang, L., Xing, W. \& Cheng, J. A microfluidic device with passive air-bubble valves for real-time measurement of dose-dependent drug cytotoxicity through impedance sensing. Biosens. Bioelectron. 32, 300-304 (2012).

29. Hua, S. Z., Sachs, F., Yang, D. X. \& Chopra, H. D. Microfluidic Actuation Using Electrochemically Generated Bubbles. Anal. Chem. 74, 6392-6396 (2002).

30. Xia, Y. \& Whitesides, G. M. SOFT LITHOGRAPHY. Annu. Rev. Mater. Sci. 28, 153-184 (1998).

31. Ebara, M., Hoffman, J. M., Hoffman, A. S. \& Stayton, P. S. Switchable surface traps for injectable bead-based chromatography in PDMS microfluidic channels. Lab Chip 6, 843 (2006).

32. Gao, J., Zhao, J., Liu, L. \& Xue, W. Dimensional effects of polymer pillar arrays on hydrophobicity. Surf. Eng. 32, 125-131(2016). 
33. Wenzel, R. N. Resistance of solid surfaces tow etting by water. Industrial \& Engineering Chemistry, 28(8), 988-994 (1936).

34. Cassie, A. B. D. \& Baxter, S. Wettability of porous surfaces. Trans. Faraday Soc. 40,546-551 (1944).

35. He, B., Patankar, N. A. \& Lee, J. Multiple Equilibrium Droplet Shapes and Design Criterion for Rough Hydrophobic Surfaces. Langmuir 19, 4999-5003 (2003).

36. Yeo, J. \& Kim, D. S. The effect of the aspect ratio on the hydrophobicity of microstructured polydimethylsiloxane (PDMS) robust surfaces. Microsyst. Technol.16,1457-1463(2010).

37. Chibbaro, S. et al. Capillary filling in microchannels with wall corrugations: a comparative study of the concus-finn criterion by continuum, kinetic, and atomistic approaches. Langmuir (2009). doi:10.1021/la901993r

38. Saha, A. A., Mitra, S. K., Tweedie, M., Roy, S. \& McLaughlin, J. Experimental and numerical investigation of capillary flow in SU8 and PDMS microchannels with integrated pillars. Microfluid. Nanofluidics7, 451-465(2009).

39. Mognetti, B. M. \& Yeomans, J. M. Capillary filling in microchannels patterned by posts. Phys. Rev. E80, 056309 (2009).

40. Kusumaatmaja, H., Pooley, C. M., Girardo, S., Pisignano, D. \& Yeomans, J. M. Capillary filling in patterned channels. Phys. Rev. E 77, 067301 (2008).

41. van der Wijngaart, W., Chugh, D., Man, E., Melin, J. \& Stemme, G. A Low-Temperature Thermopneumatic Actuation Principle for Gas Bubble Microvalves. J. Microelectromechanical Syst. 16, 765-774 (2007).

42. Pelton, R. Poly(N-isopropylacrylamide) (PNIPAM) is never hydrophobic. J. Colloid Interface Sci. 348, 673-674 (2010). 


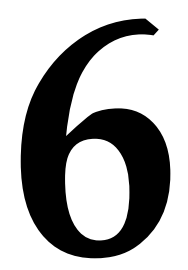

\section{High Efficiency Hydrodynamic DNA Fragmentation in a Bubbling System}


DA fragmentation down to a precise fragment size is important for biomedical applications, diagnosis of diseases, gene therapy, and shotgun sequencing. In this w ork, a cheap, easy to operate, and high-efficiency DNA fragmentation method is demonstrated based on hydrodynamic shearing in a bubbling system. We find that hydrodynamic forces generated during the bubbling process shear the DNA molecules, extending and breaking them at the points where shearing forces are larger than the strength of the phosphate backbone. Factors of applied pressure, bubbling time, and temperature have been investigated. Genomic DNA was reproducibly fragmented down to controllable 1-10 Kbp fragment lengths with a yield of $75.30-91.60 \%$. We demonstrate that the ends of the genomic DNA molecules generated from hydrodynamic shearing can be ligated by T4 ligase and the fragmented DNAs can be used as templates for polymerase chain reaction. Therefore, in the bubbling system, DNA molecules could be hydrodynamically sheared to produce smaller pieces of dsDNA available for further processes. The system could potentially serve as a DNA sample pretreatment technique in the future.

tThis chapter is based on the publication: L. Li., M. Jin, C. Sun., X. Wang., S. Xie,, G. Zhou, A. van den Berg, J.C.T. Eijkel \& L. Shui. High efficiency hydrodynamic DNA fragmentation in a bubbling system. Scientificreports, 2017, 7(1), 1-9. 
High Efficiency HydrodynamicDNA Fragmentation in a Bubbling System

\subsection{INTRODUCTION}

The size of target DNA fragments is a key parameter for next-generation sequencing (NGS) technology.1,2 DNA has a double helical strand structure storing genetic information. ${ }^{3}$ The identity and sequence of the nucleotides (A, T, C, G) define natural species and individuals, which, consequently, makes DNA sequencing a fundamental research area to extract this genetic information.45 Obtain random and size-controlled DNA fragments is a key step in the nextgeneration sequencing sample preparation process and gene expression studies. In molecular diagnostics, sections of genes are screened within a sample using various detection methods, where the target gene hybridizes to a complementary probe molecule. Therefore, DNA fragmentation is an important sample pretreatment step for diagnostic applications where short fragments are required for fast hybridization and high sensitivity target detection.

Methods available for DNA fragmentation include enzymatic digestion, ${ }^{6-9}$ sonication, ${ }^{10-12}$ nebulization, ${ }^{13,14}$ and hydrodynamic shearing. ${ }^{15-21}$ These methods have been widely used to produce DNA fragments for different applications. Each of them has its advantages and disadvantages. Enzymatic digestion using restriction endonucleases to fragment DNA in restriction enzyme cutting sites, which is efficient and precise; however, the resulting fragments are not randomly chopped, and some DNAs with high G-C content or tightly packed DNAs cannot be fully enzy matically digested.12,16 Sonication, nebulization and hydrodynamic shearing are all physical fragmentation methods. Compared to enzymatic digestion, they are more random and better controlled in size and size distribution.22 Sonication is an efficient and easy method; how ever, it can be quite variable and difficult to achieve correct size-distribution, tending to cause breaks within AT-rich regions and cause damage to DNA molecules. ${ }^{1222}$

In both nebulization and point-sink shearing, DNA molecules are fragmented by hydrodynamic shearing forces. The sheared fragment length depends on tube diameter, shape, and in-tube flow velocity. 16,17,21 Nebulization generates random DNA fragments by forcing a DNA solution through a small orifice of a nebulizer. ${ }^{13,14}$ It is a fast and reproducible method to fragment DNA molecules into small pieces; however, the resulting DNA fragments have a wide size range, and it also requires a large amount of input DNA and expensive equipment.2 Fragmentation based on hydrodynamic shearing in fluidic tubes/channels has been reported, and can produce DNA fragments of short fragment length and narrow size-distribution withless DNA damage, ${ }^{21,22}$ however, the process needs 
a complex device and well-trained operators. High efficiency, good repeatability, and low-cost technologies to achieve precise length DNA fragments are still highly required in the standard DNA sample preparation procedure for routine diagnostic processes.

In biotechnological processes, it has been found that exposure to a bubbling system can induce cell damage or protein denaturation. ${ }^{23-25}$ The bubbling process typically includes three important processes: bubble formation, bubble movement, and bubble bursting. Shear stresses are generated during the bubbling process, such as resulting from gas-liquid friction, drainage during bubble film change, and hydrodynamic forces during bubble bursting. The timescale for foam (bubble) formation and bursting is typically in the submillisecond range, ${ }^{26}$ and the shear stress generated in the process of a bubble rising through the liquid, foam draining, and bubble bursting are respectively estimated to be in the range of $10^{0}, 10^{-1}$ and $10^{3} \mathrm{~N} / \mathrm{m}^{2}$ assuming air bubble diameter of $0.5 \mathrm{~cm}$ and a film thickness of $10 \mu \mathrm{m}$ in pure water at room temperature. ${ }^{25}$ These shear stresses would act on the molecules in the system and affect their behavior.

In this work, we investigated the DNA fragmentation in a bubbling fluidic system. When gas is introduced into the DNA solution via a tube, bubbles form at the orifice, rising, merging, and bursting at the air-liquid surface. ${ }^{27,28}$ The hy drodynamic forces generated during this process arefound to break the DNA molecules into smaller pieces. By controlling gas pressure, bubbling time, and temperature, we have obtained size-controllable DNA fragments ranging from 10 to $1 \mathrm{Kbp}$ with a narrow size distribution. Ligation and RAPD-PCR experiments using fragmented genomic DNA samples confirmed the usability of the fragmented DNAs in genome sequencing and gene expressing analysis. We show that this hydrodynamic shearing DNA fragmentation method can fragment different DNA samples to desired lengths. The demonstrated method has several advantages. Except for the setup itself (Figure 6.1), there is no other chemical or biological materials involved in this bubbling system. The setup is simple and cheap to construct and oper ate in a general laboratory. The total cost of our setup is about 60 US dollars, and costs per sample is only from the gas consumption depending on the bubbling pressure and time. 
High Efficiency HydrodynamicDNA Fragmentation in a Bubbling System

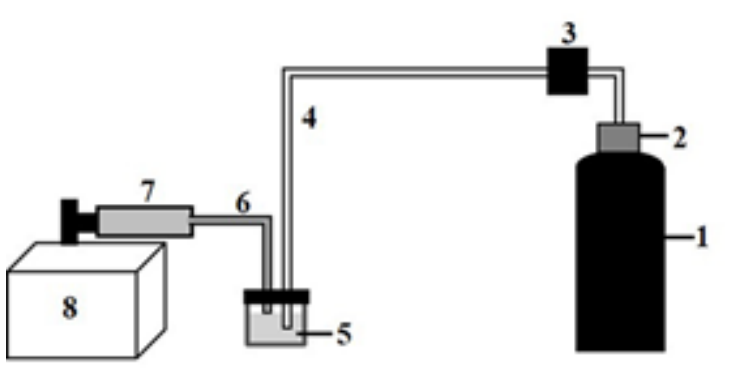

Figure 6.1. Schematic drawing of the experimental setup. 1 - $\mathrm{N}_{2}$ container, 2 - compression release valve, 3 - pressure controller, 4 - Teflon tube, 5 - DNA solution in Teflon cuvette, 6 - buffer supplier tube, 7 - syringe, 8 - syringe pump.

\subsection{MATERIAL AND METHODS}

\subsubsection{The bubbling system}

The schematic drawing of the bubbling system is shown in Fig. 1. Nitrogen gas (99.9999\% pure) is released from a $\mathrm{N}_{2} \operatorname{tank}(1)$ via a pressure release valve (2) and pressurecontroller (3) through a Teflon tube (4) to the DNA solution in a cuvette (5). To avoid solution loss and to keep the solution volume constant, a syringe pump (Gemini, KDscientific, America) (8) is used to continuously replenish the buffer solvent via a syringe (7) through a Teflon tube (6) to the sample solution (5). All accessories are disinfected in a high-pressure steam sterilizer (TOMY, SX500, Japan) before use. Gas bubbles are generated and dilated at the tip of the tube (4), then leave the tube and rise to the air-liquid interface where they burst and release the $\mathrm{N}_{2}$ to air. All materials and tools used in our experiments, including Teflon tube, cuvette, and connectors were thoroughly cleaned and sterilized before using to minimize external DNA contaminations. Experiments were carried out in a laminar flow clean workbench which had been sterilized using $70 \%$ ethanol and ultraviolet light.

\subsubsection{DNA sample preparation}

Salmon sperm DNAs (deoxy ribonucleic acid sodium salt from salmon testes, CAS: 68938-01-2, Sigma-Aldrich, Shanghai, China), herring sperm DNAs (deoxy ribonucleic acid sodium salt from herring testes, CAS: 438545-06-3, SigmaAldrich, Shanghai, China), lambda DNAs (duplex DNA, the molecular weight is $31.5 \times 106$ daltons and it is 48502 base pairs in length, CAS: B600011, Sangon Biotech, Shanghai, China) were chosen as the DNA samples for experiments. Solid samples w ere dissolved in sterilized ultra-pure w ater $(>18.25 \mathrm{M} \Omega \mathrm{cm})$ in a 
plastic cuvette at a concentration of $2.50 \mathrm{mg} / \mathrm{mL}$ and stored at $-20^{\circ} \mathrm{C}$. When used, the DNA solution was thawed and diluted to a concentration of $100 \mu \mathrm{g} / \mathrm{mL}$ with $0.5 \times$ TE-Buffer $(\mathrm{pH}=8.0)$ (diluted from $5 \times$ TE-Buffer $(\mathrm{pH}=8.0)$ containing $54.00 \mathrm{~g} / \mathrm{L}$ Tris-base), $27.50 \mathrm{~g} / \mathrm{L} \mathrm{H}_{3} \mathrm{BO}_{3}$, and $0.001 \mathrm{~mol} / \mathrm{L}$ Ethylene diamine tetra-acetic acid (EDTA). All other chemicals were purchased from Aladdin Industrial Corporation, Shanghai, China.

\subsubsection{DNA quantification and analysis}

DNA solutions and loading buffer of $0.25 \%$ bromophenol blue and $40.00 \% \mathrm{~m} / \mathrm{v}$ sucrose solution (both purchased from Aladdin Industrial Corporation, Shanghai, China) were loaded onto $100 \mathrm{~mL} 1.50 \sim 2.00 \%$ agarose gels (SigmaAldrich, CAS: $9012-36-6$, China) containing 5 uL $0.50 \mathrm{mg} / \mathrm{mL}$ ethidium bromide. Electrophoretic separation was carried out in a gel electrophoresis apparatus (DYY-8c, Beijing Liuyi Co., Ltd., Beijing, China) at a constant voltage of $\sim 115 \mathrm{~V}$. Length of the DNA fragments was computed by digitizing agarose slab gels with the Gel Imaging System (BIO-BEST "A" series, SIM, America). The image was calibrated by using marker solutions with known DNA fragment size (all markers used in our experiments were purchased from TaKaRa Biotechnology (Dalian) CO., Ltd, China), and then a cubic spline interpolation function was used to map digitized coordinates to fragment length in base pairs.

\subsubsection{Ligation Reaction}

The ligation reaction was performed in $10 \mu \mathrm{L}$ ligation reaction mixture: $1.2 \mu \mathrm{g}$ DNA sample, $1 \mu \mathrm{L}$ T4 DNA Ligase (350 U/ $\mu \mathrm{L}$, T4 DNA Ligase Kit, TaKaRa Biotechnology (Dalian) Co., Ltd, China), $1 \mu \mathrm{L}$ 10×T4 DNA Ligase buffer (T4 DNA Ligase Kit, TaKaRa Biotechnology (Dalian) Co., Ltd, China), and sterilized distilled water (added to tune the total volume to $10 \mu \mathrm{L}$ ). The mixture was incubated for $36 \mathrm{~h}$ at $15^{\circ} \mathrm{C}$ for the ligation reaction. Ligated DNAs were separated by agarose gels with the corresponding fragmented DNAs before and after the ligation reaction.

6.2.5 Random-amplified polymorphic DNA polymerase chain reaction (RAPDPCR)

RAPD-PCR w as performed to check the possibility of the fragmented DNAs from the bubbling system as DNA templates for PCR amplification. A microplate reader (Infinite M200 Pro, TECAN, Sw itzerland) w as used to determinate the DNA concentration, and $200 \mathrm{ng}$ of DNAs w as used in each RAPD-PCR reaction. 
High Efficiency HydrodynamicDNA Fragmentation in a Bubbling System

Four 10-base RAPD-PCR primers were used, primer 287 (5'-GCAACGGCGG-3'), primer C1 (5'-TTCGAGCCAG-3'), primer K2 (5'-GTCTCCGCAA-3') and primer F12 (5'-ACGGTACCAG-3'). Primer 287 has been used previously37, and all primers 287, C1，K2, F12 were provided by BGI tech (Shen Zhen, China). Three different PCR reactions $(\mathrm{x}, \mathrm{y}$, and $\mathrm{z})$ were carried out, respectively. Reaction $\mathrm{x}$ includes primers 287 and $C 1$, reaction y includes primers 287 and $K 2$, and reaction $\mathrm{z}$ includes primers 287 and F12. The RAPD-PCR reaction was: $95^{\circ} \mathrm{C}$ for $5 \mathrm{~min}$ followed by 40 cycles of $95^{\circ} \mathrm{C}$ for $45 \mathrm{~s}, 36^{\circ} \mathrm{C}$ for $1 \mathrm{~min}$, and $72^{\circ} \mathrm{C}$ for $2 \mathrm{~min}$; and $72{ }^{\circ} \mathrm{C}$ for an additional $10 \mathrm{~min}$ in a PCR Cycler (T100 Thermal Cycler, BioRad, USA).

\subsection{RESULTS AND DISCUSSION}

When $\mathrm{N}_{2}$ gas is introduced in the DNA solution via the tube, bubbles are created, rising to the solution surface and bursting there causing shearing. In this work, we have studied the effects of bubbling time, applied gas pressure, and temperature on the average fragmented DNA length.

\subsubsection{The effect of gas Pressure}

Long DNA fragments can be sheared and fragmented by the hydrodynamic forces produced in the bubbling process acting on the molecules. We have investigated the applied gas pressure effect on the DNA fragmentation results using the bubbling system. The applied pressure was $0.05,0.10,0.15,0.20,0.25$, and $0.30 \mathrm{MPa}$, and the bubbling time of $60 \mathrm{~min}$ was used to ensure maximal fragmentation at that pressure. Figure 6.2 shows that the average fragment length varies with applied gas pressure. With the increase of gas pressure, the average fragment length decreases dramatically from more than $10 \mathrm{~Kb}$ pdown to about $1 \mathrm{Kbp}$, the fragmented DNAs show a narrow distribution according to the fragmentation distribution curves (Figure E1 in Appendix E). An average fragment length of $1,090 \mathrm{bp}$ was obtained at $0.30 \mathrm{MPa}$, which we expect can be further decreased by using a better-sealed system allowing higher applied pressures. Each experiment was repeated four times, and as can be seen from Figure 6.2 that the bubbling system was stable and showed good repeatability. When the bubbling pressure $\mathrm{w}$ as increased to $>0.3 \mathrm{MPa}$ or using a smaller glass vessel, smaller DNA fragments could be obtained (Figure E3 in Appendix E); how ever, the vessel sealing cap was broken quickly. Therefore, these data of smaller DNA fragments at higher pressure are not shownhere. 


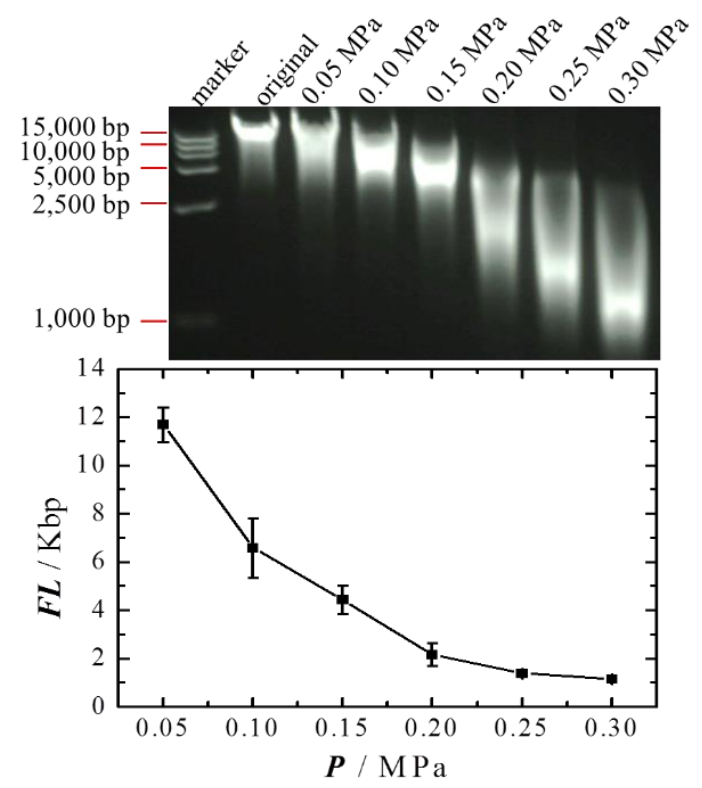

Figure 6.2. DNA frag ment length (FL) varies with applied gas pressure (P). The standard deviation is calculated from 4 repeated experiments for each point.

\subsubsection{The effect of Bubbling time}

The probability that a molecule undergoes sufficient extension to be fragmented will also be related to the number of times the molecule is ex posed to a shearing field. In the bubblingsystem, the frequency at which a DNA molecule is sheared depends on the number of bubbles which can be generated per unit vessel volume. At a fixed gas pressure and tube size, the bubble generation frequency and bubble size are constant; therefore, the bubbling time is the determining factor.

Figure 6.3 shows the effect of bubbling time on fragmentation. We fixed the gas pressure at different values and kept bubbling in DNA solution in the Teflon cuvette. A drop of DNA solution $(20 \mu \mathrm{L})$ was taken from the cuvette every $10 \mathrm{~min}$, and the fragment length was measured in electrophoresis experiments together with the original non-sheared sample for comparison. It clearly shows that the fragment length initially decreases with bubbling time, and reaches a plateau after some time. The fragment length at time 0 is not shown in the curve because the genomic DNA molecules are longer than $15 \mathrm{Kbp}$ and cannot be precisely measured using our setup. The plateau implies that DNA molecules cannot be 
further fragmented, and thus indicates the minimum length that can be achieved at that pressure. The time to reach the plateau $\left(t_{\mathrm{m}}\right)$ decreases with increasing applied gas pressure $(P)$, as shown in Figure 6.3c. It takes 55, 60, 50, 45 and 40 min to reach the plateau respectively at $0.10,0.15,0.20,0.25$ and $0.30 \mathrm{MPa}$.
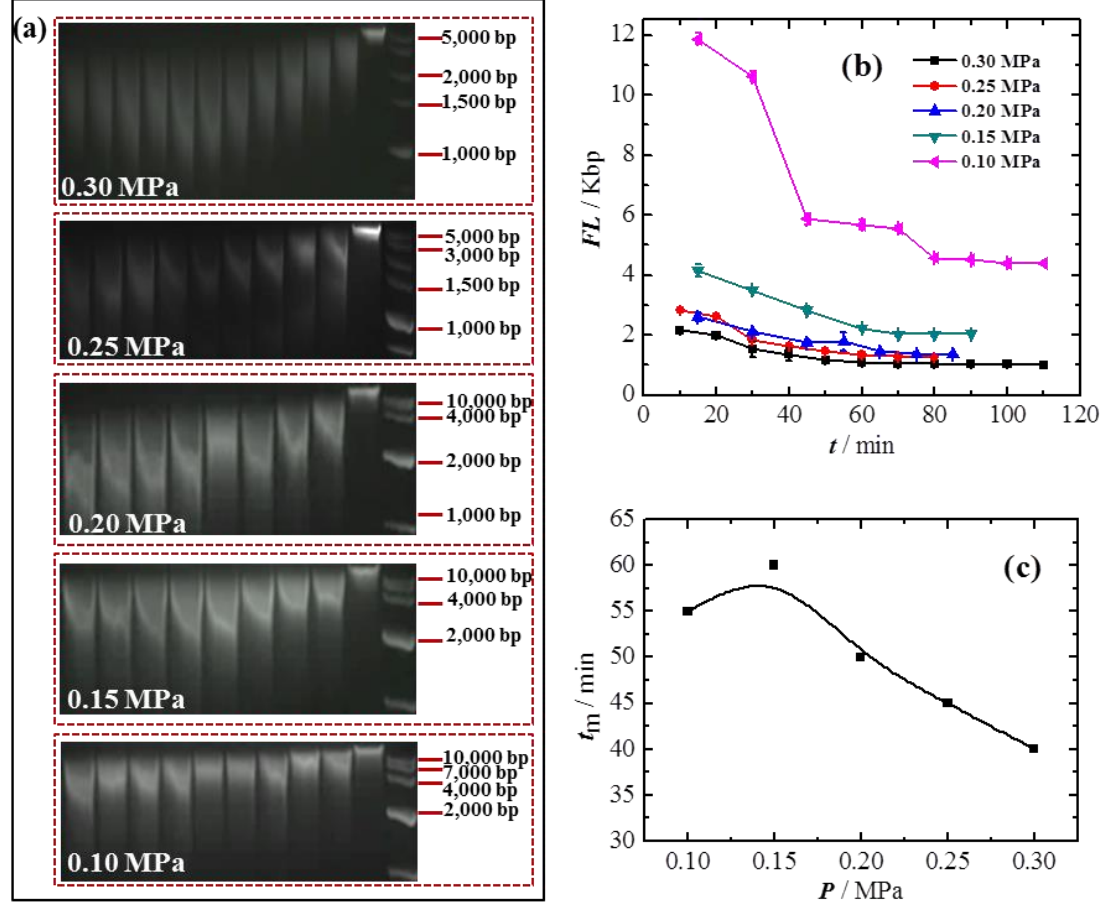

Figure 6.3. DNA fragment length varies with bubbling time at different gas pressure. (a) Gel images ofDNA fragments were obtained at different pressure and time. (b) Curves of bubbling time ( $t$ ) versus DNA fragment length at different pressure. (c) The time to reach the plateau of minimum fragment length $\left(t_{\mathrm{m}}\right)$ varies with applied gas pressure. The standard deviation is calculated from 3 to 4 repeated experiments for each point.

\subsubsection{Temperature effect}

Temperature affects the conformation of DNA molecules and viscosity of DNA solution. ${ }^{27}$ Both effects may influence the bubble formation and DNA fragmentation in our experiments. To investigate this influence, we have carried out experiments at an applied pressure of $0.25 \mathrm{MPa}$ and a bubbling time of 60 min at $0,10,20,30$, and $40^{\circ} \mathrm{C}$, as shown in Figure 6.4. Temperatures of $0-40^{\circ} \mathrm{C}$ were chosen to avoid freezing and denaturation of the DNA, of which singlechain molecules will be generated ${ }^{29}$. As shown in Figure 6.4, with increasing temperature, shorter DNA fragments were obtained. The size distribution of 
DNA fragments sheared under different temperatures is shown in Figure E2 in Appendix E.

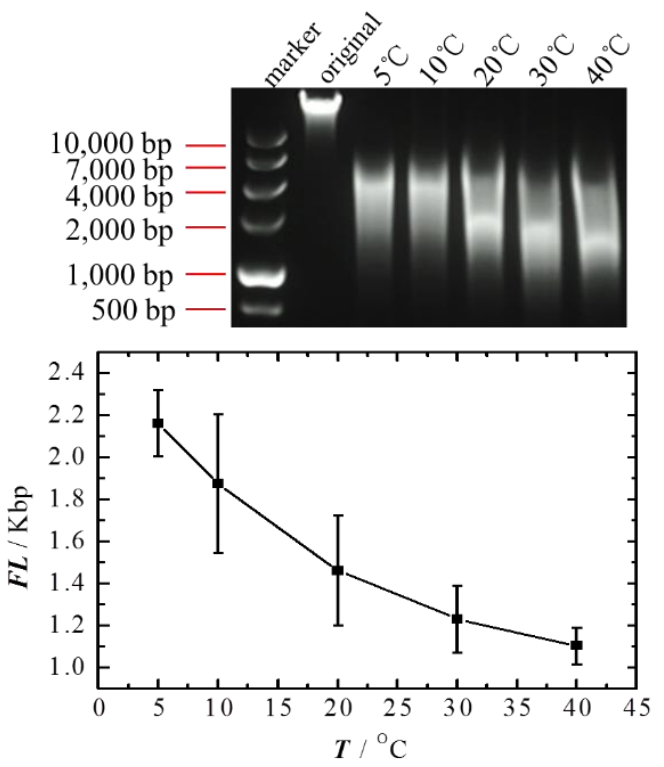

Figure 6.4. Temperature $(T)$ versus average DNA frag ment leng th obtained at the applied pressure of $0.25 \mathrm{MPa}$ and bubbling time of $60 \mathrm{~min}$. The standard deviation is calculated from 3 repeated experiments for each point.

Since viscosity decreases with increasing temperature, bubble formation and bubble rise become easier. In hydrodynamic DNA shearing systems, the fragment length increases with temperature in the same range as investigated here or decreasing the viscosity of the DNA solutions. ${ }^{16}$ This suggests that in our system the shearing forces generated during bubble formation and bubble rise do not significantly contribute to the DNA fragmentation effect. Therefore we expect that the bubble bursting might generate the major hydrodynamic forces for DNA fragmentation. On the other hand, the DNA molecular conformation and chain flexibility change with temperature. Flexible DNA molecules can be more easily extended on the bubble film, and exposed to larger shearing forces, which might also play a role in the observed increased DNA fragmentation. ${ }^{14,25}$

\subsubsection{Process Yield}

Sample loss is a problem for some DNA sample treatment methods $11,14,19-21$. In this bubbling DNA fragmentation process, water evaporates quickly and DNA sample loss can occur due to sample splashed out and stuck on tube/cuvette 
High Efficiency HydrodynamicDNA Fragmentation in a Bubbling System

surface. Therefore, we designed and applied a continuous buffer supply system to replenish the solution, keeping the sample volume constant, as shown in Fig. 1. A Teflon cuvette has been chosen because of its hydrophobic surface to avoid sample sticking. The yield of the process was calculated by

$$
\text { Yield }=\frac{m_{\mathrm{DNA}}(\text { after fragmentation })}{m_{\mathrm{DNA}}(\text { before fragmentation })} \times 100 \%
$$

Here mDNA is the amount of DNA obtained by multiplying the sample volume with the DNA concentration measured using a microplate reader (Infinite M200 Pro, TECAN, Switzerland).

As shown in Figure 6.5, the yield varies in the range of $75.30-91.60 \%$ at the applied pressures of $0.05-0.30 \mathrm{MPa}$ and bubbling time of $60 \mathrm{~min}$. Higher yield is achieved at lower gas pressure, and we expect it can be further improved with better-sealed devices. Typically, a yield of $75 \%$ could be guaranteed, which is sufficient for most genomic analysis studies.

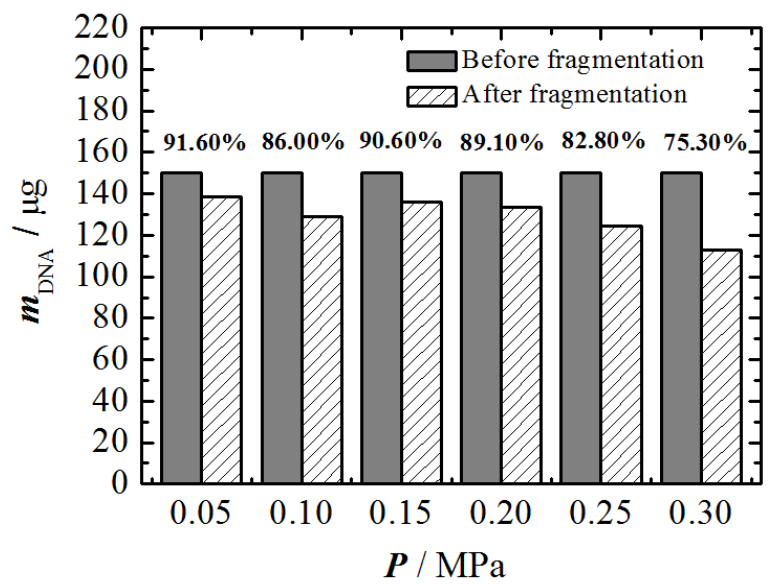

Figure 6.5. Sample yield sheared under different pressures. The bubbling time is $60 \mathrm{~min}$.

\subsubsection{Ligation of Fragmented DNA}

The ligation reaction was carried out to check the status of the ends of the fragmentedDNAs. Since there are papers reported that hydrodynamic shear and heat may cause double-strand DNA (dsDNA) to denature into single-strand DNA (ssDNA)30,31. The fragmented DNA samples were first checked by introducing ssDNA-specific nuclease, Exonuclease I (TaKaRa Biotechnology (Dalian) Co., Ltd, China), which can digest ssDNA but not dsDNA. Results from 
agarose gel electrophoresis indicate that the fragmented DNA samples were mainly dsDNA since the majority of the DNAs remain intact after the treatment with Exonuclease I (Figure E4 in Appendix E). The ability of Exonnulease I to digest ssDNA was confirmed here. Additionally, DNA concentration was also measured before and after Exonuclease I digestion using a spectrophotometer (Thermo Scientific NanoDrop 2000, USA ) (Table S1). After digestion, the DNA concentration is slightly decreased, which is acceptable considering the multiple sample treatment steps. Moreover, the ratio of optical density (OD) at 260 and $280 \mathrm{~nm}$ w avelength (OD260/OD280) is in the range of 1.8-1.9 before digestion, demonstrating that the DNA samples are quite pure without obvious contamination from RNA, protein, or phenol; however, the OD260/OD280 decreased to $<1.6$ after digestion, likely due to the presence of enzymes in the reaction solution ${ }^{32}$.

DNAs sheared under different gas pressure were ligated by T4 DNA ligase in a $10 \mu \mathrm{L}$ ligation reaction mixture, and the ligated DNAs were separated by agarose gel electrophoresis (Figure 6.6). Results demonstrate that the length of DNAs after ligation is about twice their corresponding DNAs before ligation, suggesting that the relatively smaller fragmented DNA molecules are ligated into larger ones. These results show that the ends of the fragmented DNAs are not damaged by hydrodynamic shearing and they are us able for the next step process.

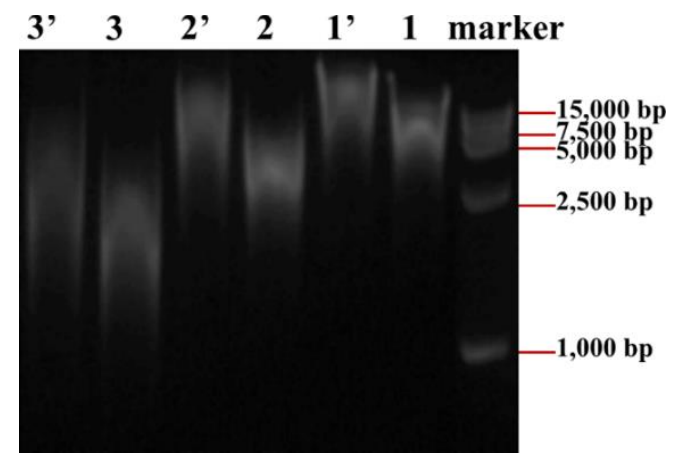

Figure 6.6. Gel images of the DNAs before and after ligation reaction with 1 and $1^{\prime}$ fragmented genomic DNAs at 0.10 MPa before and after ligation, 2 and 2'- fragmented genomic DNAs at $0.20 \mathrm{MPa}$ before and after ligation, 3 and 3 ' - fragmented genomic DNAs at $0.30 \mathrm{MPa}$ ) before and after ligation. The bubbling time is $60 \mathrm{~min}$ for all samples. Molecular weight marker DL 15,000 was used to measure the size of the DNAs. 
High Efficiency HydrodynamicDNA Fragmentation in a Bubbling System

Furthermore, two genomic DNA samples (DNAs from Salmon Testes and DNAs from Herring Testes) and one Lambda DNA w ith known A-Trich regions ${ }^{33}$ were hydrodynamically sheared using this method (Figure E5). All three samples show similar smear bands, suggesting the hydrodynamic shearing in the bubbling system is un-selective for A-T rich region at the conditions applied in our experiments.

\subsubsection{RAPD-PCR}

Amplification of random regions of genomic DNA using 10-base primers has been done to check the fragmented DNAs as templates for PCR. Three RAPDPCR reactions were performed for each of the two fragmented DNA samples (sample No. 2:30 min at 0.20 MPa; sample No.3:30 min at 0.30 MPa). Genomic DNAs without fragmentation were used as positive controls (sample No. 1) (Figure 6.7(a)). The average fragment length of samples 1, 2, and 3 are $>15 \mathrm{Kbp}$, $\sim 2.5 \mathrm{Kbp}$, and $\sim 1.2 \mathrm{Kbp}$, respectively. All three DNA samples can be successfully amplified in three different RAPD-PCR reactions, generating products with specific bands in the range of 1,000 to 5,000 b pin size (Figure 6.7(b)).

For smaller size PCR products $(<1.5 \mathrm{~kb})$, the band patterns were very similar for the fragmented and non-fragmented DNA molecules. For larger size PCR products $(>3 \mathrm{~kb})$, as expected, weaker bands were observed due to the reduced size of the DNA template in samples 2 and 3 . These results suggest that the fragmented genomic DNAs can be used for PCR amplification, and we expect they can also be used for DNA hybridization, mate-pair sequencing, shotgun sequencing, genome polymorphism analysis, DNA microarray, paternity test, and so on.

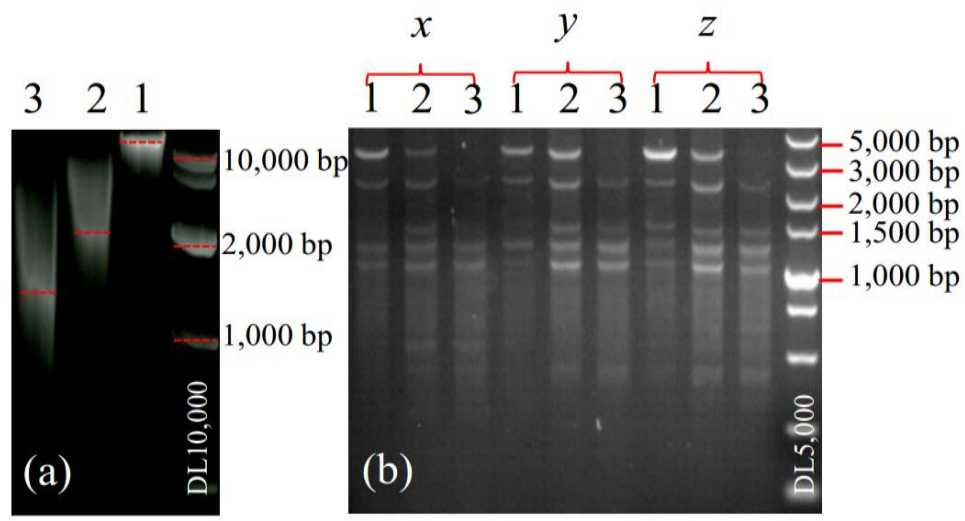


Figure 6.7. RAPD-PCR experimental results. (a) DNA samples used for RAPD-PCR experiments. 1 - untreated genomic DNAs, positive control; 2 - fragmented genomic DNAs (30 min at $0.20 \mathrm{MPa}$ ), 3 - fragmented genomic DNAs (30 min at $0.30 \mathrm{MPa}$ ). Molecular weight marker DL 10,000 was used to estimate the size of the genomic DNAs. (b) RAPD-PCR products of sample 1,2, 3 in three different reactions using different sets of primers - $(x, y, z)$, and molecular weight marker DL 5,000 was used to estimate the size of the PCR products.

In summary, genomic DNAs can be efficiently and unselectively fragmented to dsDNA by hydrodynamic shearing with fragment ends available for ligationand fragments as templates for PCR.

\subsubsection{Discussion}

When $\mathrm{N}_{2}$ is introduced into the DNA solution via a small tube, bubbles are generated from the tube tip (orifice), and then rise to the solution surface where they burst to release $\mathrm{N}_{2}$ to air and DNA solution back to the bulk solution. A high-speed camera (Phantom Miro M110, Vision Research Inc., Wayne County, NC, USA) w as used to visualize and record the bubbling process as shown in Figure 6.8. The images were taken under a resolution of $1,280 \times 800$, and the shooting frequency was 1,600 fps. At the pressure of $0.10 \mathrm{MPa}, \mathrm{N}_{2}$ gas was released from the orifice of a $0.5 \mathrm{~mm}$ inside-diameter tube. A bubble was generated (traced in a red dashed square) (a); grew and released from the orifice (b); rose to the air/solution interface (c); coalesced $w$ ith another bubble to form a larger bubble (d); ruptured at the air-solution interface (e).

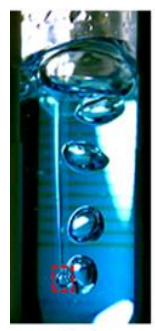

(a)

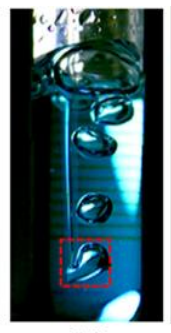

(b)

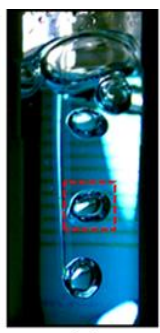

(c)

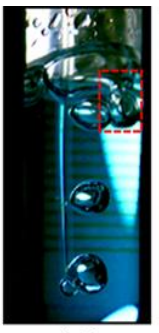

(d)

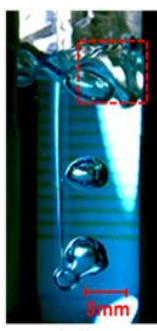

(e)

Figure 6.8. High-speed microscope images of bubbling process. (a) Bubble generation, (b) Bubble dilation and release, (c) Bubble rising, (d) Bubble coalescence with another one to a larger bubble, (e) Larger bubble rupture at the liquid-air interface. This sequence of events is also schematically shown in Figure 6.9. 


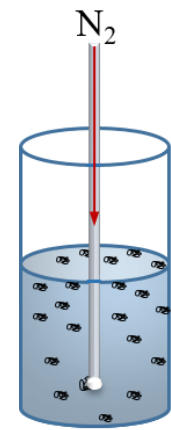

(a)

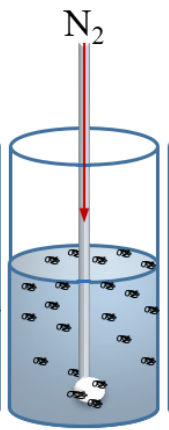

(b)

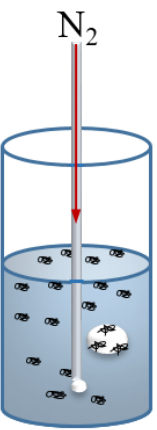

(c)

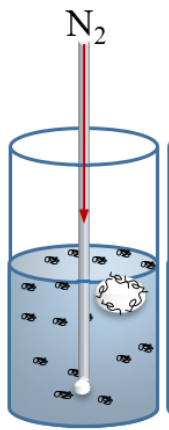

(d)

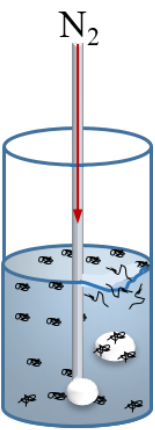

(e)

Figure 6.9. Schematic drawing of DNA fragmentation in the bubbling system. (a) Initiation of a gas bubble at the tube orifice, and DNA molecules absorbed to the bubble surface, (b) Bubble dilation, more DNA molecules to the bubble surface, (c) Bubble detaching and rising, DNA molecules move with bubble and sheared by flow around the bubble, (d) Bubble coalescence, DNA molecules sheared by the extensional strain force, (e) Bubble bursting, DNA molecules broken by bubble breakup and high-speed liquid jet.

DNA molecules are commonly coiled in the aqueous solution and can be stretched by elongational shear forces. ${ }^{34}$ Lentz et al. have investigated the mechanism of DNA fragmentation by jet nebulization and found that hydrodynamic shear is responsible for DNA degradation. ${ }^{13}$ Clarkson et. al. have confirmed that protein denaturation in foam is correlated with interfacial exposure leading to conformational changes at the gas-liquid interface. ${ }^{1425}$ When the residence time of the DNA fragments in a shear gradient is sufficiently long, it allows the extension of the DNA molecules into a highly extended conformation. When the molecule assumes a completely extended configuration the applied forces are greatest and most likely to break covalent bonds. As mentioned before, the bubble film thickness is in the range of a few to tens of micrometers, ${ }^{35}$ the timescale for foam (bubble) bursting is in the sub-millisecond range, ${ }^{26}$ and the bubble bursting at the air-solution interface generates the highest shear stress during the bubbling process. ${ }^{25} \mathrm{In}$ this process the bubble film becomes increasingly thinner leading to bubble rupture at the free gas-liquid surface with the thin liquid film breaking up into many tiny droplets generating jets of liquid that are projected into the air due to the rush of inflowing gas as the pressure in the bubble is released. 28,36

To understand the main contributions to DNA fragmentation in the bubbling system, several factors have been considered and tested. Firstly, $\mathrm{N}_{2}$ was chosen 
as the gas source of bubbles to avoid oxidation during DNA fragmentation contributing to the process. Secondly, we performed experiments by putting a layer of silicon oil on top of the DNA solution to investigate the effect of the bubble bursting on the fragmentation results. No obvious DNA fragmentation results were achieved when the DNA solution surface was covered by $1 \mathrm{~mL}$ silicon oil, while the same DNA sample could be fragmented to $\sim 8 \mathrm{Kbp}$ without the oil film under the same conditions (bubbling for $30 \mathrm{~min}$ at $0.10 \mathrm{MPa}$, Fig. S6). This points at the bubble bursting and jet formation as the main process causing DNA fragmentation. Furthermore, smaller fragments were obtained at higher temperatures as described above in section 6.3.3. As mentioned in this section, this also points to the fact that bubble formation and rising are not the main causes of DNA fragmentation. Taking all these factors into account, we conclude that DNA fragmentation in the bubbling system is mainly caused by shearing during the bubble bursting and jet formation (Figure 6.9). Multiple fragmentation events will lead to increasingly shorter fragments with the fragmentation continuing until the fragments are too short to be sufficiently extended for shear breaking at different thresholds.

\subsection{CONCLUSION}

DNA fragmentation is an important sample pretreatment step for nucleic acid analysis, which is critical for rapid hybridization reaction of gene study and the production of genomic libraries. In this chapter, we have demonstra ted a DNA fragmentation method using a home-built bubbling system. This method can efficiently fragment genomic DNA samples to the range of 1.0-10 Kbp with high reproducibility. Random and contamination-free DNA fragmentation is guaranteed according to the hydrodynamic shearing using high-purity $\mathrm{N}_{2}$ gas. The use of this method is simple, cheap, and ready-to-use for many laboratories. The fragment length can be further decreased with a better-sealed sample cuvette.

\subsection{REFERENCES}

1 Bahassi el, M. \& Stambrook, P. J. Next-generation sequencing technologies: breaking the sound barrier of human genetics. Mutagenesis 29,303-310, (2014).

2 Head, S. R. et al. Library construction for next-generation sequencing: Overviews and challenges. Biotechniques 56, 61-77, (2014).

3 Watson, J. D. \& Crick, F. H. Molecular structure of nucleic acids; a structure for deoxyribose nucleic acid. Nature 171, 737-738, (1953). 
High Efficiency HydrodynamicDNA Fragmentation in a Bubbling System

4 FrankKamenetskii, M. D. Biophysics of the DNA molecule. Phys. Rep. 288, 13-60, (1997).

5 Liu, L. et al. Comparison of next-generation sequencing systems. J. Biomed. Biotechnol. 2012, 251364,(2012).

6 Anderson, S. Shotgun DNA sequencing using cloned DNase I-generated fragments. Nucleic. Acids. Res. 9, 3015-3027, (1981).

7 Hoheisel, J. D., Nizetic, D. \& Lehrach, H. Control of partial digestion combining the enzymes dam methylase and Mbol. Nucleic. Acids. Res. 17, 9571-9582, (1989).

8 Seed, B., Parker, R. C. \& Davidson, N. Representation of DNA sequences in recombinant DNA libraries prepared by restriction enzyme partial digestion. Gene 19, 201-209, (1982).

9 Wong, K. K., Markillie, L. M. \& Saffer, J. D. A novel method for producing partial restriction digestion of DNA fragments by PCR with 5-methyl-CTP. Nucleic. Acids. Res. 25, 4169-4171, (1997).

10 Tseng, Q., Lomonosov, A. M., Furlong, E. E. \& Merten, C. A. Fragmentation of DNA in a sub-microliter microfluidic sonication device. Lab Chip 12, 4677-4682, (2012).

11 Deininger, P. L. Random subcloning of sonicated DNA: application to shotgunDNA sequence analysis. Anal. Biochem. 129, 216-223, (1983).

12 Kasoji, S. K. et al. Cavitation Enhancing Nanodroplets Mediate Efficient DNA Fragmentation in a Bench Top Ultrasonic Water Bath. PLoS One 10, e0133014, (2015).

13 Lentz, Y. K., Worden, L. R., Anchordoquy, T. J. \& Lengsfeld, C. S. Effect of jet nebulization on DNA: identifying the dominant degradation mechanism and mitigation methods. J. Aerosol. Sci.36, 973-990, (2005).

14 Sambrook, J. \& Russell, D. W. Fragmentation of DNA by nebulization. CSH Protoc. 2006, pdb.prot4539, (2006).

15 Yew, F. F. \& Davidson, N. Breakage by hydrodynamic shear of the bonds between cohered ends of lambda-DNA molecules. Biopolymers 6, 659$679,(1968)$.

16 Thorstenson, Y.R., Hunicke-Smith, S.P., Oefner, P.J. \& Davis, R. W. An automated hydrodynamic process for controlled, unbiased DNA shearing. Genome Res. 8, 848-855, (1998).

17 Shui, L. L. et al. High yield DNA fragmentation using cyclical hydrodynamic shearing. Rsc Adv.3, 13115-13118, (2013).

18 Oefner, P. J. et al. Efficient random subcloning of DNA sheared in a recirculating point-sink flow system. Nucleic. Acids. Res. 24, 3879-3886, (1996). 
19 Nesterova, I. V., Hupert, M. L., Witek, M. A. \& Soper, S. A. Hydrodynamicshearing of DNA in a polymeric microfluidic device. Lab Chip 12, 1044-1047, (2012).

20 Joneja, A. \& Huang, X. H. A device for automated hydrodynamic shearing of genomic DNA. Biotechniques 46, 553-556, (2009).

21 Shui, L., Bomer, J. G., Jin, M., Carlen, E. T. \& van den Berg, A. Microfluidic DNA fragmentation for on-chip genomic analysis. Nanotechnology 22, 494013, (2011).

22 Hengen, P. N. Shearing DNA for genomic library construction. Trends Biochem. Sci. 22, 273-274, (1997).

23 Chalmers, J. J. Cells and Bubbles inSparged Bioreactors. Cy totechnology 15,311-320, (1994).

24 Michaels, J. D., Mallik, A. K. \& Papoutsakis, E. T. Sparging and agitationinduced injury of cultured animals cells: Do cell-to-bubble interactions in the bulk liquid injure cells? Biotechnol. Bioeng. 51,399-409, (1996).

25 Clarkson, J. R., Cui, Z. F., Darton, R. C. \& Clarkson, J. R. Protein denaturation in foam - I. Mechanism study. J. Colloid Interface Sci. 215, 323-332, (1999).

26 Wilde, P.J. Interfaces: their role in foam and emulsion behaviour. Curr. Opin. Colloid Interface Sci. 5, 176-181, (2000).

27 Xu, Y. G., Ersson, M. \& Jonsson, P. G. A Mathematical Modeling Study of Bubble Formations in a MoltenSteelBath. Metall. Mater. Trans. B. 46, 2628-2638, (2015).

28 Boulton-Stone, J. M. \& Blake, J. R. Gas bubbles bursting at a free surface. J. Fluid Mech. 254, 437-466, (1993).

29 SantaLucia, J., Jr. \& Hicks, D. The thermodynamics of DNA structural motifs. Annu. Rev. Bioph. Biom. 33, 415-440, (2004).

30 Hershey, A. D., Goldberg, E., Burgi, E. \& Ingraham, L. Local denaturation of DNA by shearing forces and by heat. Journal of molecular biology 6, 230-243, (1963).

31 Pyeritz, R. E., Schlegel, R. A. \& Thomas, C. A., Jr. Hydrodynamic shear breakage of DNA may produce single-chained terminals. Biochimica et biophysica acta 272, 504-509, (1972).

32 Yantz, G. R. et al. Rapid DNA stretching in sudden elongational shear flow. Biophys. J. 86, 327a-327a, (2004).

33 Han, Y. B. \& Shikazono, N. The effect of bubble acceleration on the liquid film thickness in micro tubes. Int, J, Heat Fluid Fl.31, 630-639, (2010).

34 Lord, R. Some application of photographic. Nature 44, 249-254, (1891).

35 Huang, C. F., Lin, Y.H. \& Chen, J.D. The use of RAPD markers to assess catfish hybridization. Biodivers. Conserv. 14,3003-3014, (2005) 


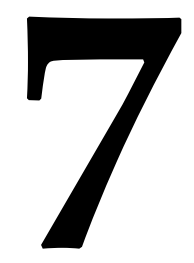

Summary and Outlook 
This chapter summarizes the main results and conclusions presented in this

1 thesis. Recommendations for improvement of the developed devices and suggestion for future direction are provided as outlook. 


\subsection{SUMMARY OF CHAPTERS 2-5 OF THE THESIS}

In this thesis, PDMS-based microfluidic devices functionalized with PNIPAm have been developed for droplet generation and capillary flow manipulation. The working principles of these devices were explained based on the timedependent w etting behavior of $w$ ater or aqueous solution s on PNIPAm-g-PDMS surfaces. The switchable wettability was used for reversible and multiform emulsion generation in droplet-based devices and to create stop valving in capillary-based devices. Moreover, the capillary filling behavior surprisingly showed a constant filling flow rate, which was studied and explained using a modified Washburn equation taking into account the diffusive hydration of PNIPAm in w ater at liquid front. The device w as successfully used for alcohol sensing in beer. By subsequently integrating air gates in the capillarity-based microfluidic devices, a device with more complete functionality was obtainedfor controlling capillary flow.

Chapter 2 described the surface coating method based on photo-grafting of the thermo-responsive polymer, PNIPAm, onto PDMS. This coating method also serves as a basic coating technology in chapters 3,4 , and 5 . A reversible thermoresponsive wettability was achieved. When varying the PNIPAm-g-PDMS surface temperature between 25 and $38{ }^{\circ} \mathrm{C}$, the surface wettability can be reversibly controlled between hydrophilic $\left(\mathrm{CA}=26^{\circ}\right)$ to hydrophobic $\left(\mathrm{CA}=106^{\circ}\right)$. The dynamic tuning of the channel surface wettability enabled us to obtain reversible $\mathrm{O} / \mathrm{W}$ and $\mathrm{W} / \mathrm{O}$ emulsions in the same chip. O/W/O and W/O/W double emulsions could be formed by either locally coating the channel surface with PNIPAm or locally heating the PNIPAm grafted channel. The devices w ere used to produce core-shell microcapsules with different shell thicknesses. This device can serve as a useful platform to study dynamical processes of reversible multiphase micro/nano-fluidic phenomena on surfaces or in confined microspaces.

In chapter 3 , the same grafting technology was used in a PDMS-based capillary microfluidic device. Strong time-dependent wetting behavior of water on PNIPAm-g-PDMS surface was observed and investigated by contact angle measurements. The reversible wettability was used to create a stop valve to control the capillary flow, as water fills the channel automatically by capillary forces and can be stopped by locally controlling the surface wettability of the channel walls by switching the temperature from $20^{\circ} \mathrm{C}$ to $36^{\circ} \mathrm{C}$. Moreover, in this device constant capillary filling flow rates (non-Washburn capillary filling 
behavior) ranging from $1 \mu \mathrm{m} / \mathrm{s}$ to $240 \mu \mathrm{m} / \mathrm{s}$ w ere observed. We explained this phenomenon by diffusive hydration of PNIPAm in water at the meniscus. When diffusive hydration dominates, we will obtain constant filling flow rates in the channel. When there is no diffusive hydration, the filling behavior follows Washburn equation $w$ ith filling length proportional to $\sqrt{t}$. The constant capillary filling flow rate in PNIPAm-g-PDMS devices provides a steady and controllable flow field that could be favorable for incubation process in immun oassays. It can also be used as a liquid metering platform. In a channel with a given temperature and geometry, the liquid volume only depends on filling time.

Water-PNIPAm interaction can be affected by a small amount of alcohol. In chapter 4, we investigated the wetting behavior of water/ethanol mixtures on PNIPAm-g-PDMS surface and studied the effect of the ethanol content on the filling behavior of $w$ ater/ethanol mixtures in PNIPAm-g-PDMS microchannels. Water/ethanol mixtures between $0 \%$ to $4 \%$ molar fraction show a constant filling flow rate in PNIPAm-g-PDMS microchannels with a flow rate that is dependent on the ethanol concentration. Water/ethanol mixtures with higher molar fraction show a filling behavior transition from constant flow rate to W ashburn b ehavior. In general terms, the liquid filling behavior in PNIPAm-g-PDMS channels is governed by the liquid-PNIPAm interaction, liquid surface tension, and viscosity. The dependence of the filling rate on the ethanol content makes the device a promising tool for the detection of low alcohol concentrations in alcoholic beverages by simply comparing the filling times. By using this method, Grolsch beers with $0 \%, 2 \%$, and $5 \% \mathrm{ABV}$ were distinguished from each other within $10 \mathrm{~s}$.

In chapter 5, we further improved the performance of the PNIPAm-g-PDMS stop valve. By adding free-standing pillars on its surface, we obtained a surface with a wider w ettability range. Now, a stop valving function was also obtained in freshly coated chips. The stop valve function in a capillary microfluidic device is not sufficient for programmable liquid manipulation, as the liquid flow cannot be stopped again. Therefore, we integrated air gate stop valves with PNIPAm-gPDMS stop valves in one w orking unit. The air gate can be actuated by thermal expansion of the air in air pockets located at the sides of the hydrophobic stop valve. Both the hydrophobic valve and air gate can be actuated in the temperature range of $20-38{ }^{\circ} \mathrm{C}$. A device containing 4 working units was then developed and tested with the ability for liquid manipulation such as liquid loading, liquid mixing and liquid delivery by sequentially actuating the valving units. 
Chapter 6 presents a fast, easy, and robust DNA fragmentation technology using a bubbling system. By introducing $\mathrm{N}_{2}$ gas into a DNA aqueous solution via a small tube, a bubble will form at the orifice of the tube, rising, expanding and coalescing at the liquid surface and finally bursting followed by formation of a liquid jet. During the bubble formation to the bursting process, genomic DNA is absorbed to the gas-liquid interface (bubble thin film), with the DNA extending and being sheared by the hydrodynamic shearing force generated during the bubble bursting and the jet formation. The genomic DNA was sheared to the range of 1.0-10 Kbp with high reproducibility. The method is simple, cheap, and ready-to-use for many laboratories as a sample pretreatment protocol for DNA analysis.

\subsection{RECOMMENDATIONS AND OUTLOOK}

The work presented in this thesis mainly focuses on developing microfluidic devices with thermally responsive wettability for emulsion generation and capillary flow control. Work has been done step by step, from surface functionalization to the development of a single working unit, till the integration of different working units in a single device to achieve better performance for liquid manipulation. How ever, in view of the interesting phenomenon behind the reversible wettability of PNIPAm-g-PDMS surfaces, more work is recommended for better understanding the phenomenon and thereby improve the current devices:

- The constant capillary filling flow rate of $w$ ater as observed in PNIPAmg-PDMS microchannel in chapter 3 is recommended tobe fundamentally studied using Molecular Dynamics simulations. A better understanding of the molecular interactions on the liquid-solid surface may make the liquid filling behavior more controllable;

- We recommend touse a PNIPAm thin film surface for studying the effect of surface wettability on phase inversion in confined micro-spaces;

- Air venting is recommended to be integrated into the device developed in chapter 5 to avoid stable air plug formation by the water evaporation, as discussed in section 5.3.4 of chapter 
Chapter 7

130 


\section{Appendix $A$}

In-Channel Responsive Surface Wettability for Reversible and Multiform Emulsion

Droplet Preparation and Applications 


\section{A.1 Schematic drawing of the fabrication process of PDMS device}

The detailed fabrication process of PDMS devices is presented in Figure A1, with step-by-step description of details.

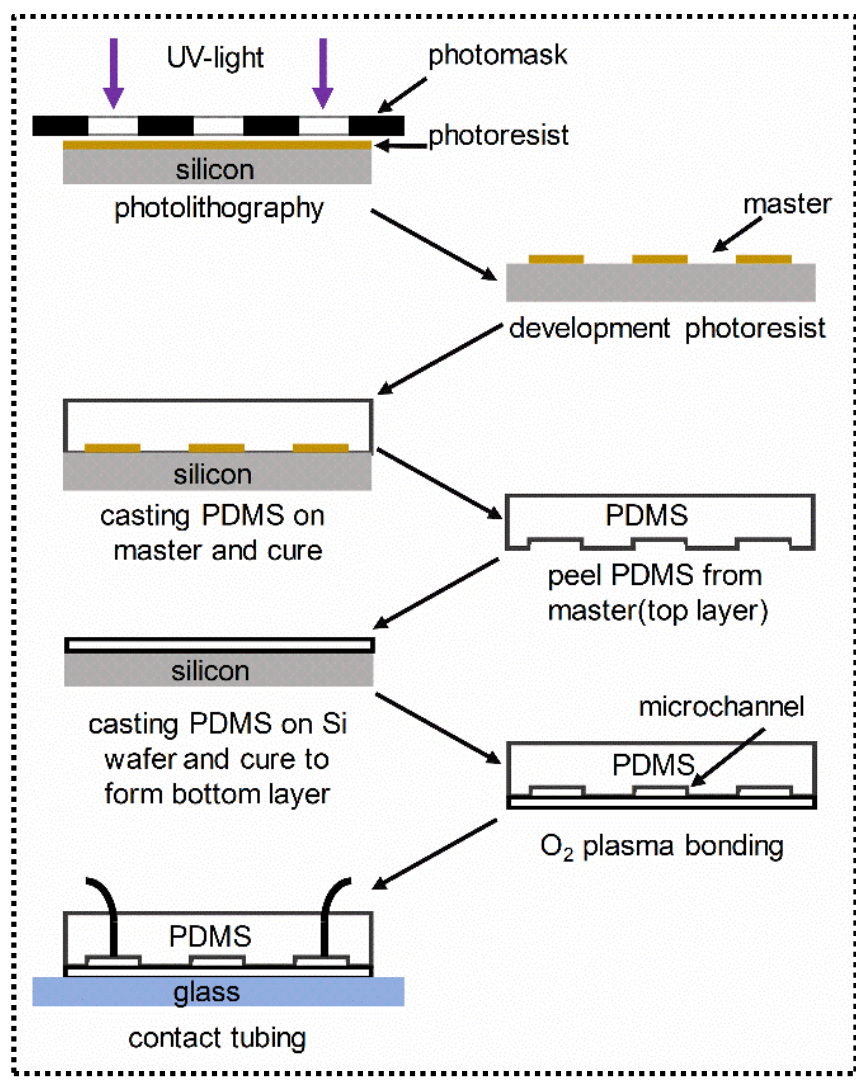

Figure A1 Schematic drawing of the fabrication process of the PDMS device.

\section{A.2 Schematic drawing of the fabrication process of patterned ITO heater}

The detailed fabrication process of the heater via patterned ITO on glass is presented in Figure A2, with step by step description of details. 


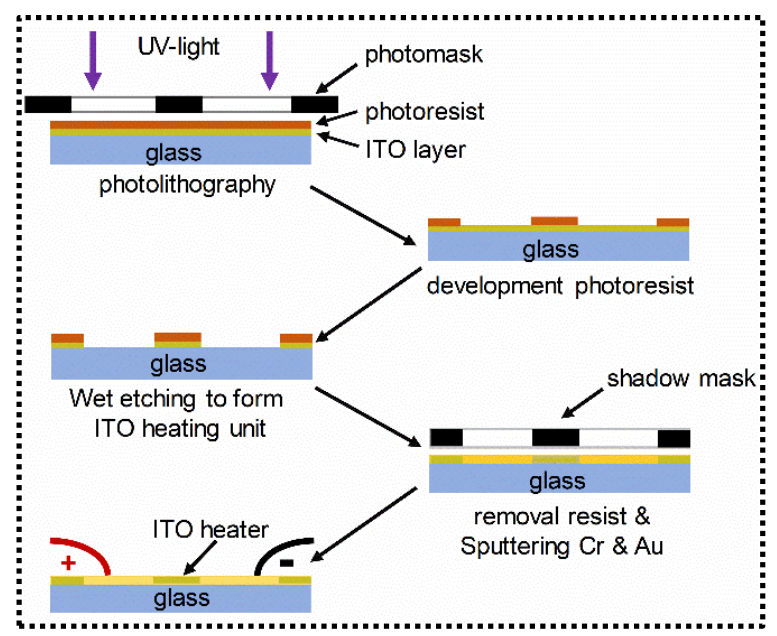

Figure A2 Schematic drawing of the fabrication process of patterned ITO heater.

\section{A.3 Long-term stability of PNIPAm coated PDMS channel}

Long-term stability of PNIPAm-g-PDMS with UV irradiation times of 5, 10, 15, 20 , and $25 \mathrm{~min}$, have been investigated by measuring the water contact angles after storage of the prepared devices in a box for a couple of days, the results are show $n$ in Figure A3. The water contact angle increased $w$ ith storage time after freshly treated, and then kept stable after one week. When the UV irradiation time w as longer than $10 \mathrm{~min}$, measured water contact angles were lower than $70^{\circ}$ after 4 w eeks.

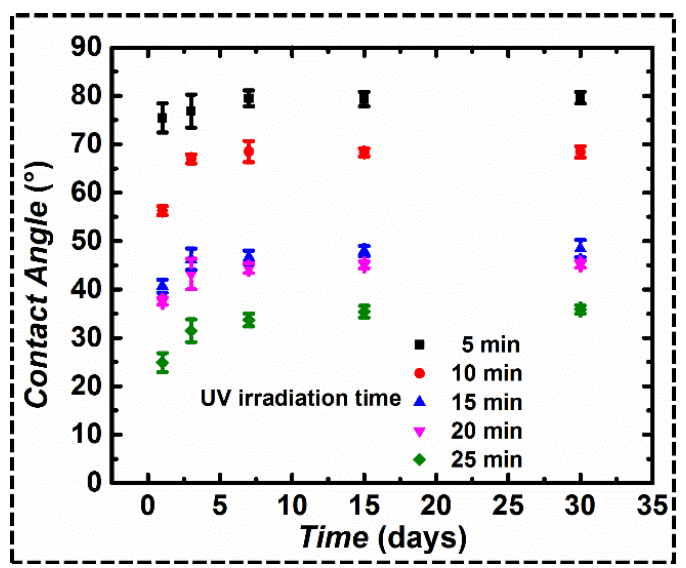

Figure A3 Long-term stability of PNIPAm-g-PDMS prepared under UV irradiation for 5, $10,15,20$, and $25 \mathrm{~min}$. The error bars represent the standard deviation calculated from 5 repeated experiments for each data point. 
A.4 Reversibility of thermo-responsive surface wettability on PNIPAm-gPDMS film

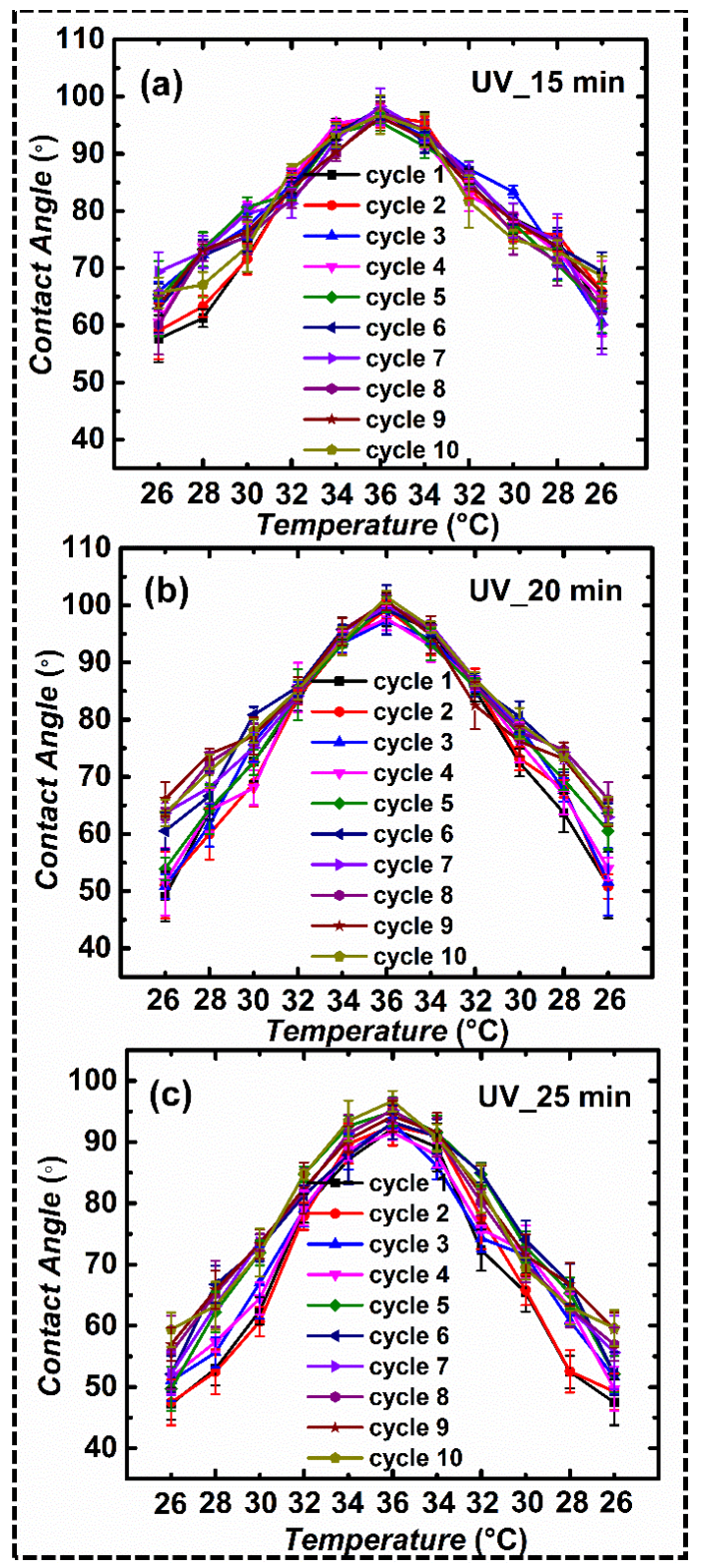

Figure A4 Reversibility of thermo-responsive surface wettability of the freshly prepared PNIPAm-g-PDMS films via different UV irradiation times. (a), (b), (c) are the curves of contact angle varying with temperature on the PNIPAm-g-PDMS surfaces prepared by UV irradiation time for 15, 20, $25 \mathrm{~min}$. 
The reversibility of thermo-responsive surface w ettability on PNIPAm-g-PDMS films $w$ as investigated by heating and cooling cycles. PNIPAm-g-PDMS films were prepared under UV irradiation time of $15,20,25 \mathrm{~min}$. The films were heated from $26{ }^{\circ} \mathrm{C}$ (around room temperature) to $36{ }^{\circ} \mathrm{C}$ (the PNIPAm-g-PDMS will become hydrophobic according to Figure 2.2b) for 10 cycles. Figure A4 shows the surface w ettability changes of the PNIPAm-g-PDMS films after heating and cooling for 10 cycles. CAs on the freshly treated films were $57 \pm 4,49 \pm 2.6$ and $46 \pm 3.7^{\circ}$ at $26^{\circ} \mathrm{C}$. After 10 cycles of heating and cooling, CAs at $26^{\circ} \mathrm{C}$ increased to $65.7 \pm 2^{\circ}, 63 \pm 2.3^{\circ}$, and $59.3 \pm 2.7^{\circ}$. The hydrophilic/hydrophobic transition temperature w as around $34-36^{\circ} \mathrm{C}$. These results exhibit the stable reversibility of surface $C A$ change for the samples prepared under different UV irradiation times.

\section{A.5 Water in oil contact angle on PNIPAm-g-PDMS surface at different temperature}

The w ater in oil contact angle on PNIPAm-g-PDMS surface in oil (CAwo) was measured at different temperatures. In this experiment, NIPAm was grafted on $1 \mathrm{~mm}$ thick PDMS film at a UV irradiation time of $15 \mathrm{~min}$. Afterward, the PNIPAm-g-PDMS film $w$ as bonded with glass and immersed in mineral oil at different temperatures. The $w$ ater in oil contact angle on PNIPAm-g-PDMS film was then measured by dripping a $1 \mu \mathrm{L}$ droplet of DI water, with experimental results are summarized in Table A1. At the temperature below $32{ }^{\circ} \mathrm{C}$ (LCST of PNIPAm), CAwo is smaller than $90^{\circ}$, the PNIPAm-g-PDMS surface w as wetting preferentially towards the w ater. At a temperature around $32{ }^{\circ} \mathrm{C}$, the PNIPAmg-PDMS surface started to become hydrophobic, and $C A$ wo increased to be about $92^{\circ}$. At the temperature higher than $32^{\circ} \mathrm{C}, \mathrm{CAwo}$ increased tobe around $104^{\circ}$ and $114^{\circ}$ at 36 and $40^{\circ} \mathrm{C}$, respectively, showing preferential w etting towards mineral oil.

Table A1 Water in oil contact angle on PNIPAm-g-PDMS surface at different temperatures.

\begin{tabular}{|c|c|c|c|c|c|}
\hline $\mathrm{T}\left({ }^{\circ} \mathrm{C}\right)$ & 25 & 28 & 32 & 36 & 40 \\
\hline $\mathrm{CA}_{\mathrm{w}}$ & $\mathrm{CA}: 63^{\circ}$ & $\mathrm{CA}: 84^{\circ}$ & $\mathrm{CA}: 92^{\circ}$ & $\mathrm{CA}: 104^{\circ}$ & $\mathrm{CA}: 114^{\circ}$ \\
& & & & & \\
\hline
\end{tabular}




\section{A.6 Local modification process of one of the FFD sections via the flow control process and the double emulsion droplets formation in corresponding devices}

To form W/O/W double emulsion droplets, the first (left) and second (right) FFD sections should be hydrophobic and hydrophilic. Thus, the channels were first pretreated with BP solution flowing through the second FFDjunction while gas flowing through the first FFD section to avoid BP solution flowing into it. The channels in the second FFD section were then filled with NIPAm monomer and irradiated with UV light for $10 \mathrm{~min}$, and then flushed and dried using DI w ater and air, respectively. The prepared device $w$ as then used to produce $\mathrm{W} / \mathrm{O} / \mathrm{W}$ double emulsion droplets (Figure A5 a(i)). The aqueous solution containing 2.0 $w \mathrm{t} \%$ PVA and $15.0 \mathrm{wt} \%$ glycerol was injected as the outermost phase at a flow rate of $14 \mu \mathrm{L} / \mathrm{min}$, the FC-40 solution containing $2.0 \mathrm{wt} \%$ PFPE-PEG600 as the surfactant $w$ as pumped into the middle channel at a flow rate of $1.6 \mu \mathrm{L} / \mathrm{min}$, and pure DI $w$ ater was used as the innermost phase and pump intothe channel at 0.6 $\mu \mathrm{L} / \mathrm{min}$. Figure $\mathbf{A} 5 \mathrm{a}$ (iv) shows the collected droplets with high enwrapping efficiency (almost 100\%). The droplet size and middle phase thickness could be tuned by varying the flow rates of different phases. The droplet diameter and size distribution of both single emulsion droplets and double emulsion droplets generated at FFD junctions 1 and 2 are shown in Figure A5 a(iv). The droplet size distribution was calculated from 200 droplets.

For the same principle, $\mathrm{O} / \mathrm{W} / \mathrm{O}$ double emulsion droplets have also been obtained, as shown in Figure A5b. The HFE-7500 solution containing $2.0 \% \mathrm{w} / \mathrm{w}$ PFPE-PEG600 as the surfactant was pumped into the external channel at a flow rate of $10 \mu \mathrm{L} / \mathrm{min}$, the aqueous solution containing $2.0 \mathrm{wt} \%$ PVA and $15.0 \mathrm{wt} \%$ glycerol $\mathrm{w}$ as injected as the middle phase at $2.2 \mu \mathrm{L} / \mathrm{min}$, and pure HFE-7500 was used as the innermost phase and injected into the inner channel at $0.6 \mu \mathrm{L} / \mathrm{min}$. Collected droplets are shown in Figure A5 b(iv). The droplet diameter and size distribution of both the single emulsion droplets of $(\mathrm{O} / \mathrm{W})$ and the double emulsion droplets of $(\mathrm{O} / \mathrm{W} / \mathrm{O})$ generated at FFDjunctions 1 and 2 are shown in Figure A5 b(iv). The droplet size distribution was calculated from 200 droplets. 

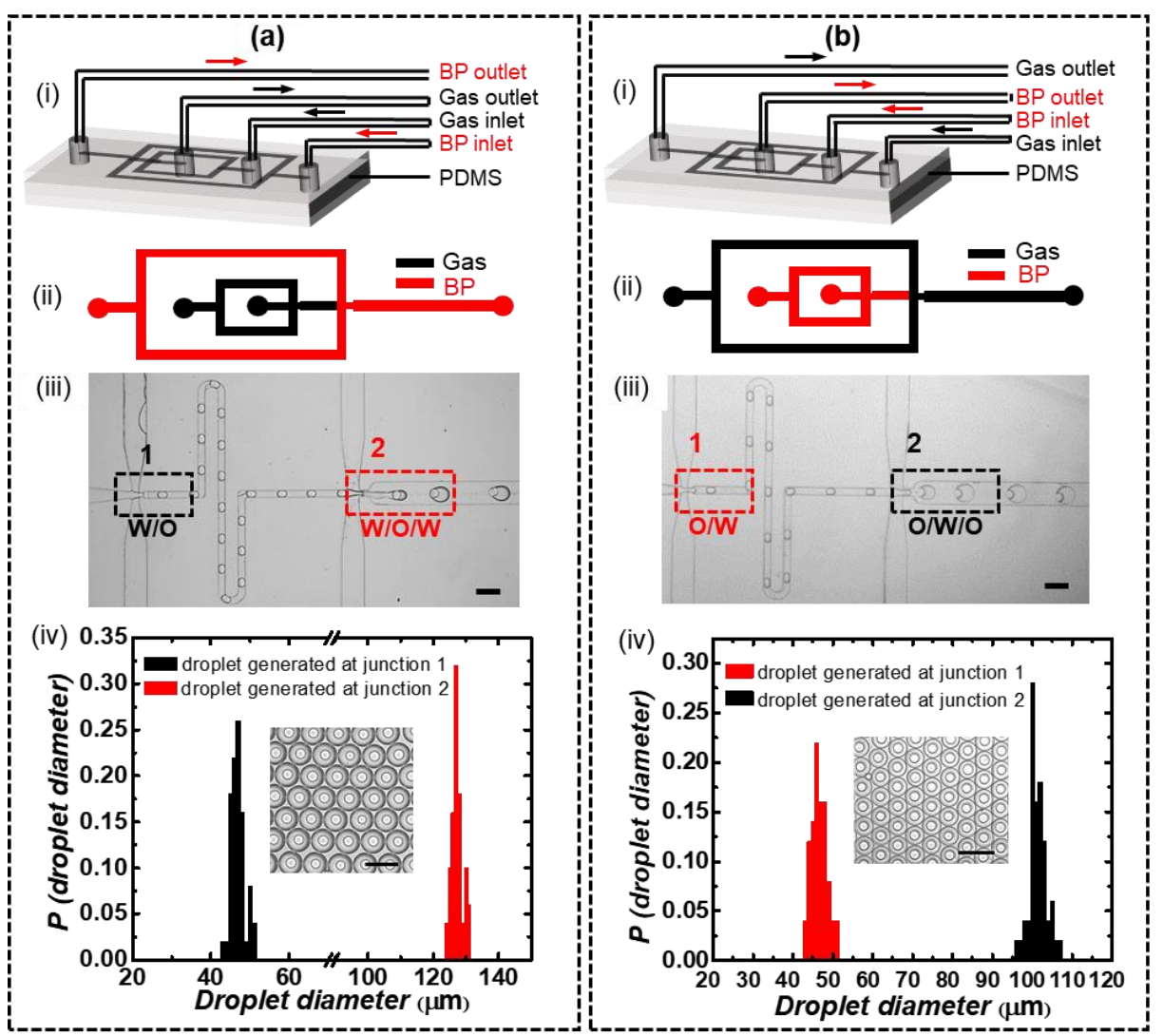

Figure A5 Local surface modification of PDMS channels via the flow process for controllable double emulsion droplet generation in microfluidic devices. (a) Local modification of the FFD section 2 for producing of $\mathrm{W} / \mathrm{O} / \mathrm{W}$ double emulsion droplets: (i) and (ii) selective hydrophilic surface coating of the FFD section 2 via the flow process (frame in red), (iii) formation of $\mathrm{W} / \mathrm{O}$ and $\mathrm{W} / \mathrm{O} / \mathrm{W}$ droplets at junctions 1 and 2, respectively, and (iv) collected droplets and the droplet diameter and size distribution generated at junctions 1 and 2. (b) Local modification of the FFD section 1 for production of O/W/O double emulsion droplets: (i) and (ii) selective hydrophilic surface coating of the FFD section 1 via the flow process (frame in red), (iii) formation of $\mathrm{O} / \mathrm{W}$ and $\mathrm{O} / \mathrm{W} / \mathrm{O}$ droplets at junctions 1 and 2, respectively, and (iv) collected droplets and the droplet diameter and size distribution generated at junctions 1 and 2 . The scale bars denote 200 $\mu \mathrm{m}$. The droplet diameter distribution was calculated with a total droplet number of 200 . 
Appendix A 


\section{Appendix}

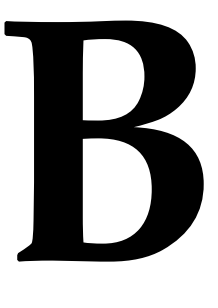

Autonomous Capillary Microfluidic Devices with Constant Flow Rate and Temperature-Controlled Valving 


\section{B.1 Temperature sensor characterization}

The tem perature sensor characterization is done as described in the experimental part. Figure B1 shows the measured resistance and linear fit of the sensor. It show s a sensitivity of $0.0495 \Omega /{ }^{\circ} \mathrm{C}$ and an offset of $20.948 \Omega$. The linearity shows an $\mathrm{R}^{2}$ value of 0.9994 .

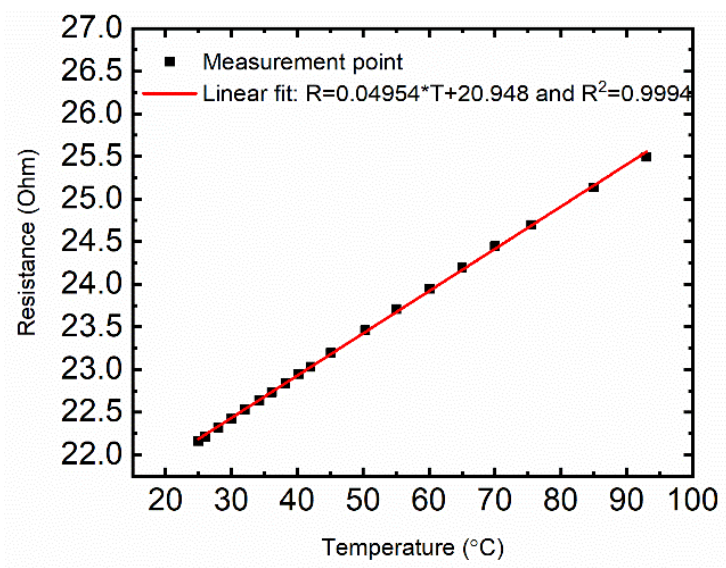

Figure B1 Temperature characteristic of the temperature sensor.

\section{B.2 UV-induced surface grafting of PNIPAm on PDMS mediated by benzophenone}

(1)

PDMS

(2)

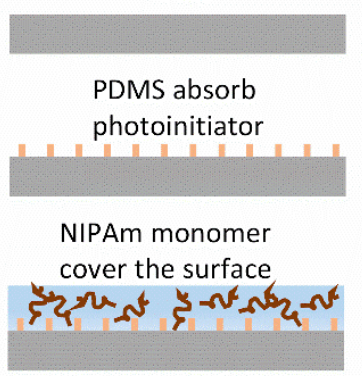

(4) UV initiated grafting and polymer chain growth
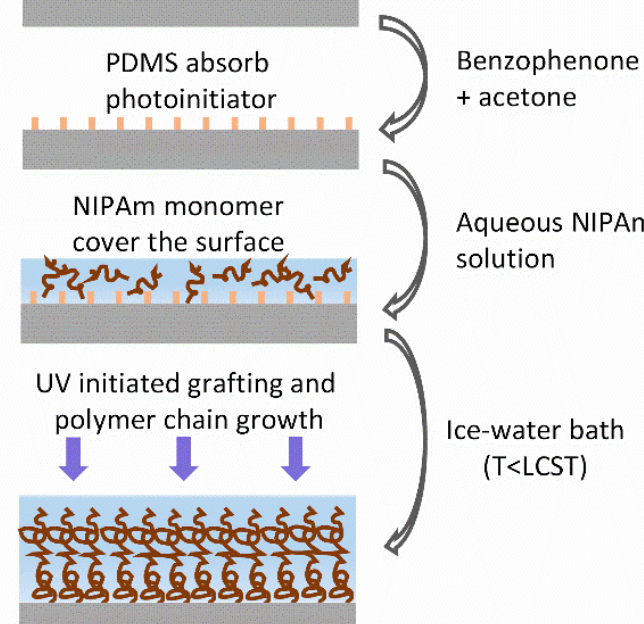

Aqueous NIPAm

solution

ce-water bath

( $\mathrm{T}<\mathrm{LCST}$ )

Figure B2 Schematic drawing of UV-induced surface grafting of PNIPAm on PDMS. 
B.3 Fluorescent dye concentration as an indicator for Benzophenone concentration in the different channel walls

We used the fluorescent Rhodamine $6 \mathrm{G}$ at an equal concentration with Benzophenone photoinitiator in acetone as a concentration indicator. The channel was filled with Rhodamine 6G-acetone for $5 \mathrm{~min}$ follow ed by a deep w ash of the channels with DI water. After w ashing, a fluorescence microscope image was captured using Olympus IX51 with a FLIR Grasshopper®3 color camera. As shown in Figure B3, results indicate that the concentration of benzophenone in the bottom PDMS layer is much higher than in the top walland sidew alls.

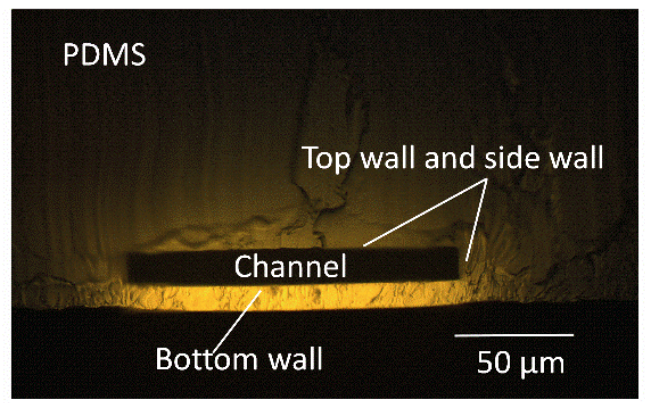

Figure B3. Fluorescent Rhodamine 6G in the PDMS channel walls.

\section{B.4 Videos of the filling behavior of water in PNIPAm-g-PDMS channel}

Videos 1, 2 and 3 show the filling behavior of DI $w$ ater in PNIPAm-g-PDMS channel with $300 \mu \mathrm{m}$ in width and 34,60,100 $\mu \mathrm{m}$ in height, respectively.

video 1

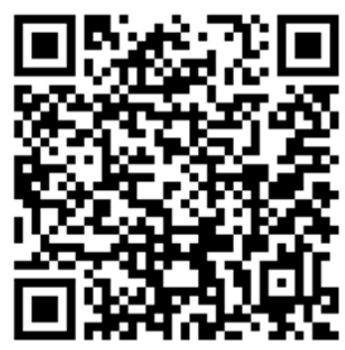

video 2

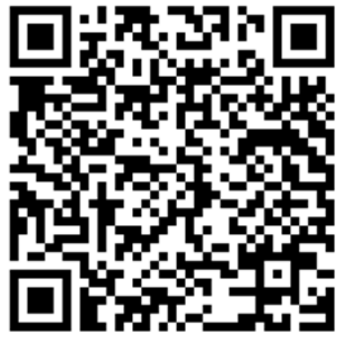

video 3

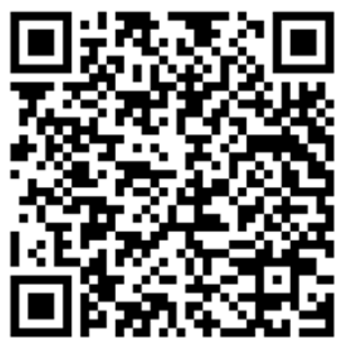

\section{B.5 Video of stop valving}

Video 4 shows the stop valving function in PNIPAm-g-PDMS channel at temperature of $40^{\circ} \mathrm{C}$. 


\section{video 4}

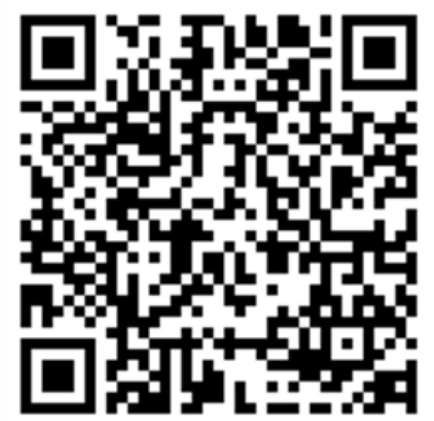

B.6 Material properties used in the numerical simulation

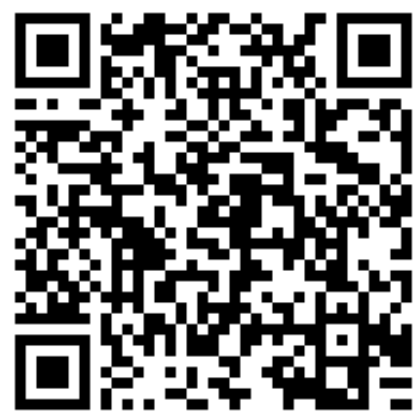

\section{B.7 References}

1. Ma, D., Chen, H., Shi, D., Li, Z. \& Wang, J. Preparation and characterization of thermo-responsive PDMS surfaces grafted with poly(N-isopropylacrylamide) by benzophenone-initiated photopolymerization. J. Colloid Interface Sci. 332, 85-90 (2009). 
Appendix

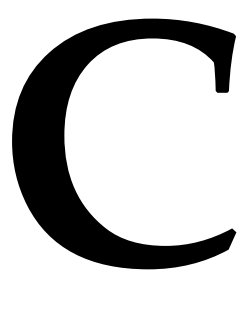

On-Chip Detection of the Alcohol Concentration in Beer 


\section{C.1 Temperature effects on the wetting behavior of DI water and water- ethanol mixtures on a PNIPAm-g-PDMS surface.}

The wetting behavior of DI water and water-ethanol mixtures with molar fraction $X$ of $0.0097,0.028,0.037,0.226$ and ethanol is shown in Figure $\mathbf{C} 1$. The observed behavior is rather complex. At temperatures below $32^{\circ} \mathrm{C}$, generally, an increase in contact angle with temperature is observed. From $32-36{ }^{\circ} \mathrm{C}$, this increase is also observed for the DI w ater and the water/ethanol mixtures with a molar fraction of up to 0.037 , while water/ethanol mixtures $w$ ith a molar fraction of 0.226 and pure ethanol show opposite changes in contact angle.
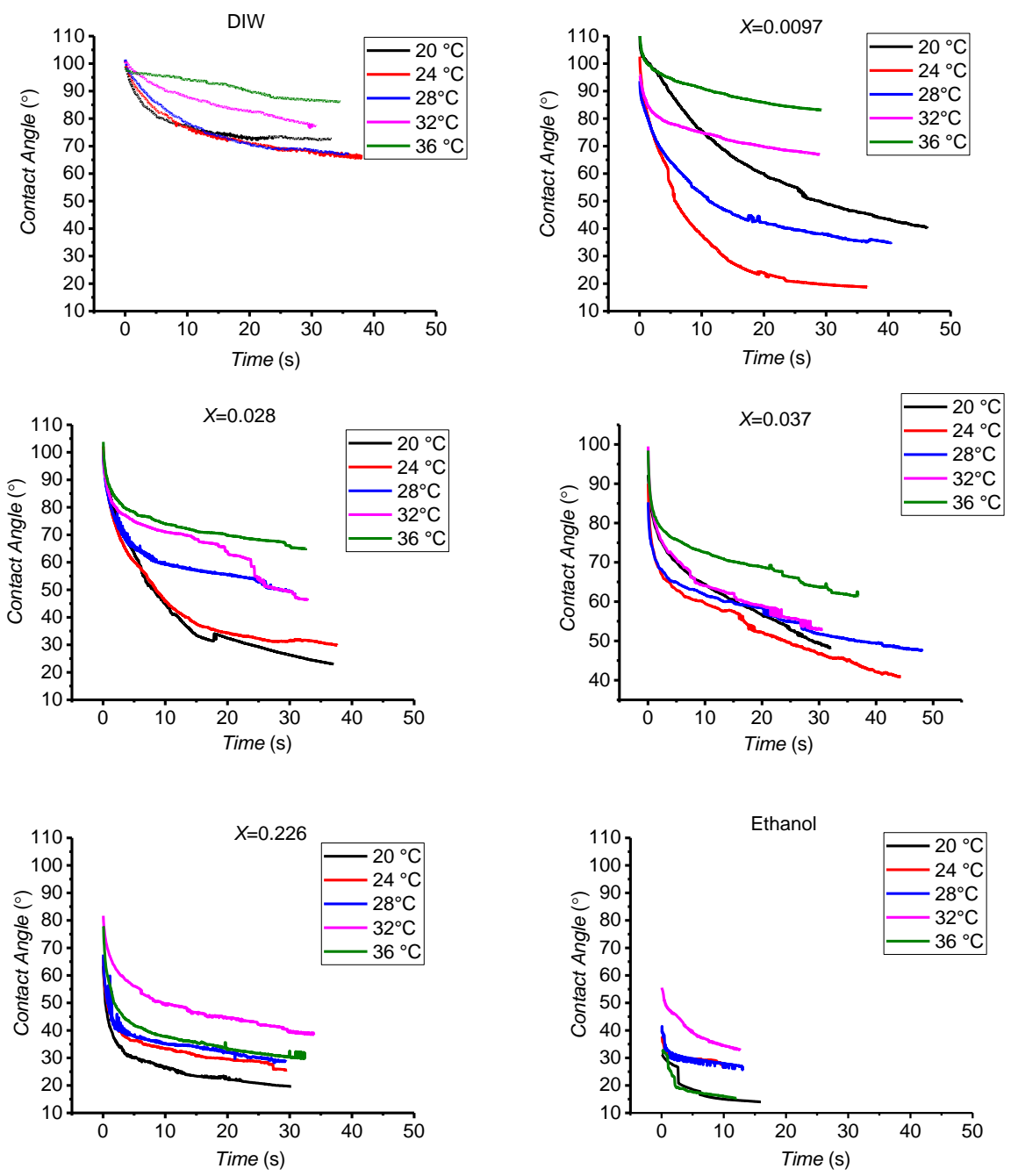
Appendix C

Figure C1 wetting property of DI water (DIW), water-ethanol mixtures with malar fraction $X=0.0097,0.028,0.037,0.226$, and ethanol on PNIPAm-g-PDMS at different temperatures. 
Appendix C

146 


\section{Appendix}

\section{Integration of Air-pockets and}

Pillared Stop Valves in

Microchannel for

Capillary Flow

Control 


\section{D.1 Mask layout for fabrication of SU-8 mold for PDMS chip fabrication}

The PDMS channel is integrated with free standing pillar arrays on the top wall. Tw o masks were used to fabricate the SU8 mold. The first mask w as designed and used to make the first layer SU-8 pattern (Figure D2). The second mask (Figure D3) was designed for fabricating the second layer SU-8 pattern with holes. A schematic overview of the fabrication process is shown in Figure D3a.

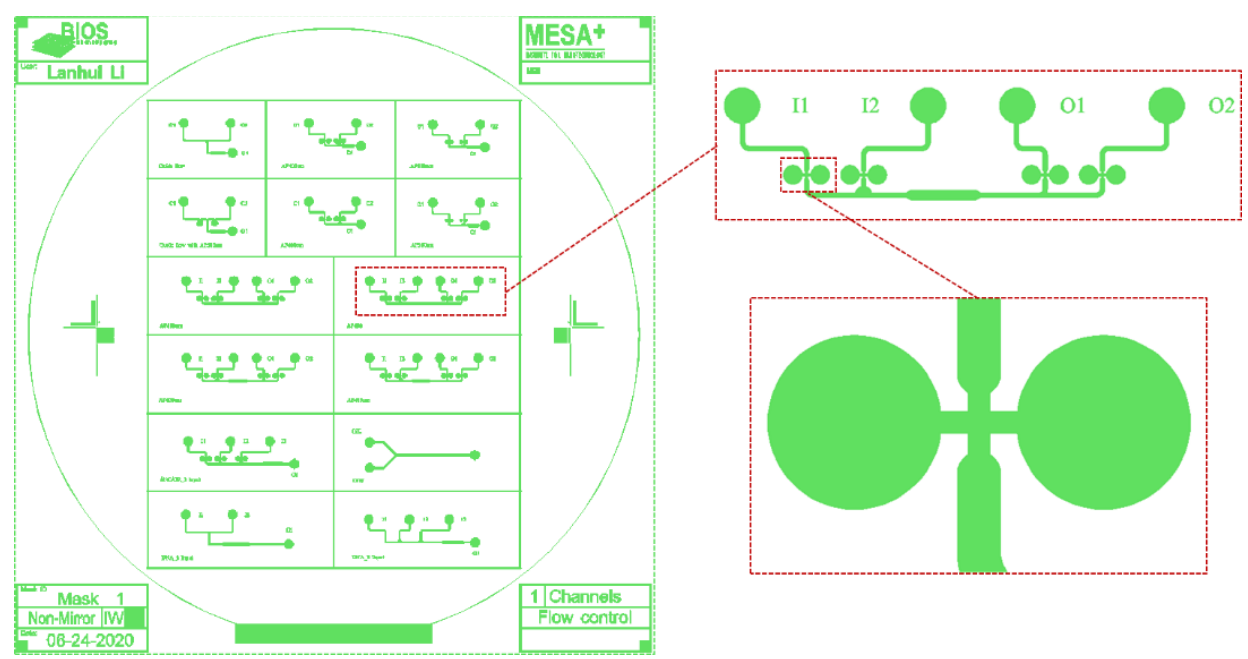

Figure D1 Mask 1 for the $1^{\text {st }}$ layerSU-8 pattern. Image on the top right shows the channel layout used in chapter 5 . Image on the bottom right presents the design of the air pockets aside from the channel.

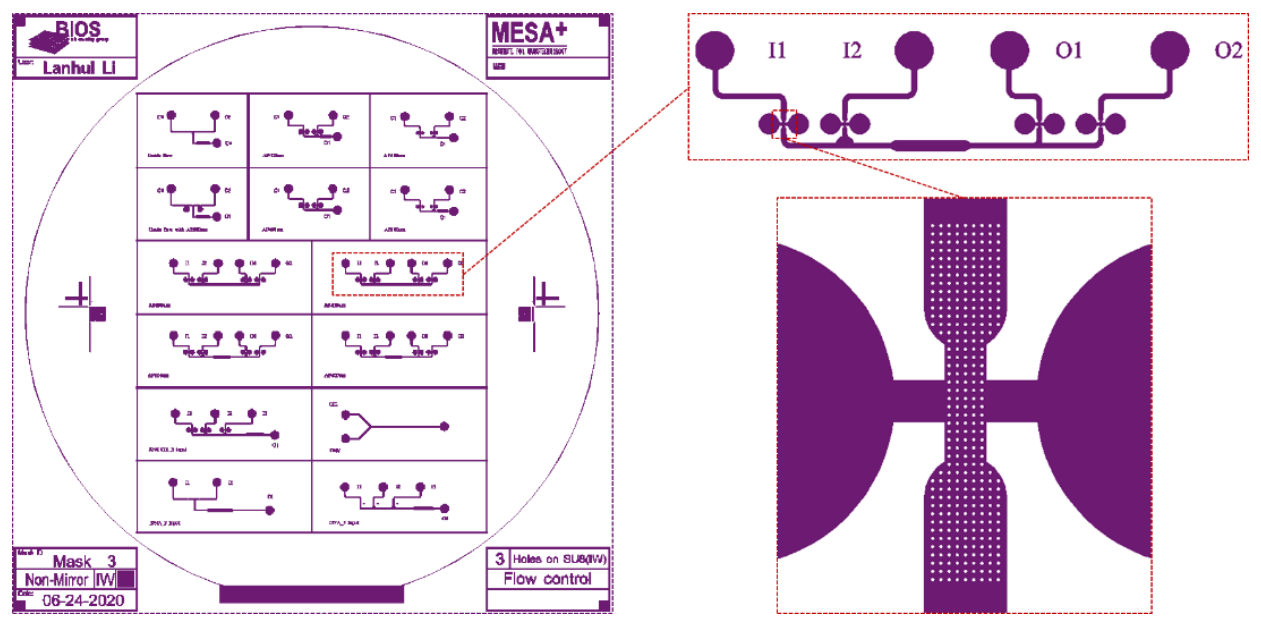


Figure D2 Mask 2 for the $2^{\text {nd }}$ layer SU-8 pattern. Image on the top right shows the channel layout which is the same as the channel layout in Figure D2. Image on the bottom right represents the mask for fabricating holes in the $2^{\text {nd }}$ layer SU-8 pattern.

\section{D.2 SU-8 mold and PDMS chip fabrication}

(a) SU8 mold fabrication

Spin coat $1^{\text {st }}$ SU8-25 \& soft bake

UV-exposure with mask 1

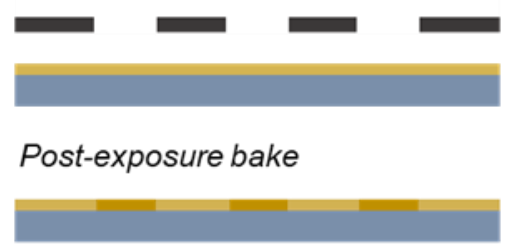

Spin coat $2^{\text {nd }}$ SU8-25 \& soft bake

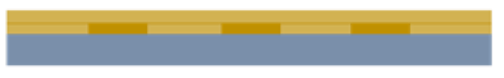

UV-exposure with mask 2

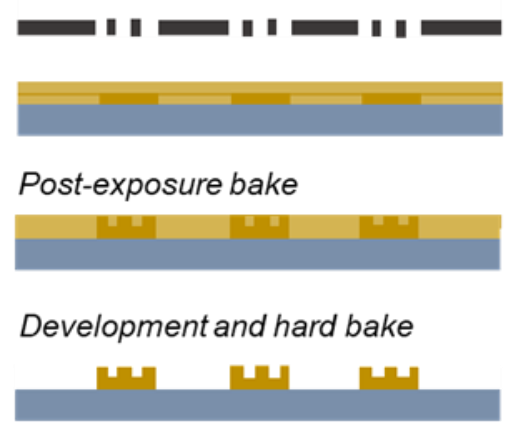

(b) PDMS chip fabrication

1. Top-layer
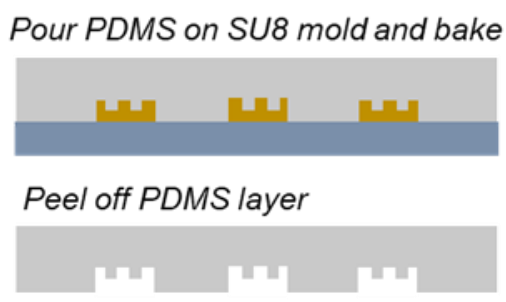

2. Bottom-layer

Spin coat PDMS on bare si-wafer

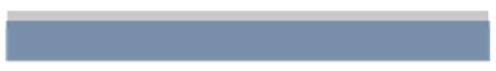

Thermo-bonding

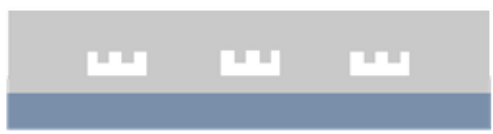

si-wafer

PDMS

Mask

SU8-25

Polymerized SU8-25

Figure D3 (a) Fabrication process ofSU-8 mold using masks in Figure D2. (b) Fabrication of PDMS chip integ rated with air-pockets and free-standing pillars.

\section{D.3 PDMS chip with air pockets and free-standing pillars}


(a)

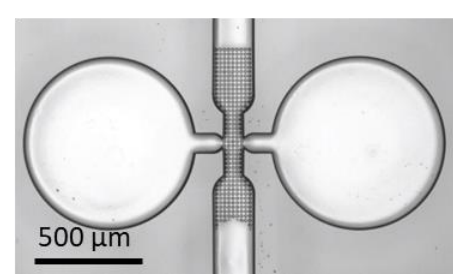

(b)

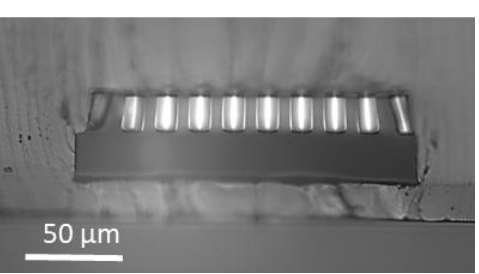

Figure D4 (a) Optical microscopy image of air-pockets and locally pillared PDMS microchannel. (b) Cross-section of the pillared PDMS channel.

\section{D.4 Mask layout of heaters and sensors}

Figure D1 shows the mask layout used for fabrication of the platinum heaters and temperature sensors on the glass wafer. The design was made using CleWin 5 .

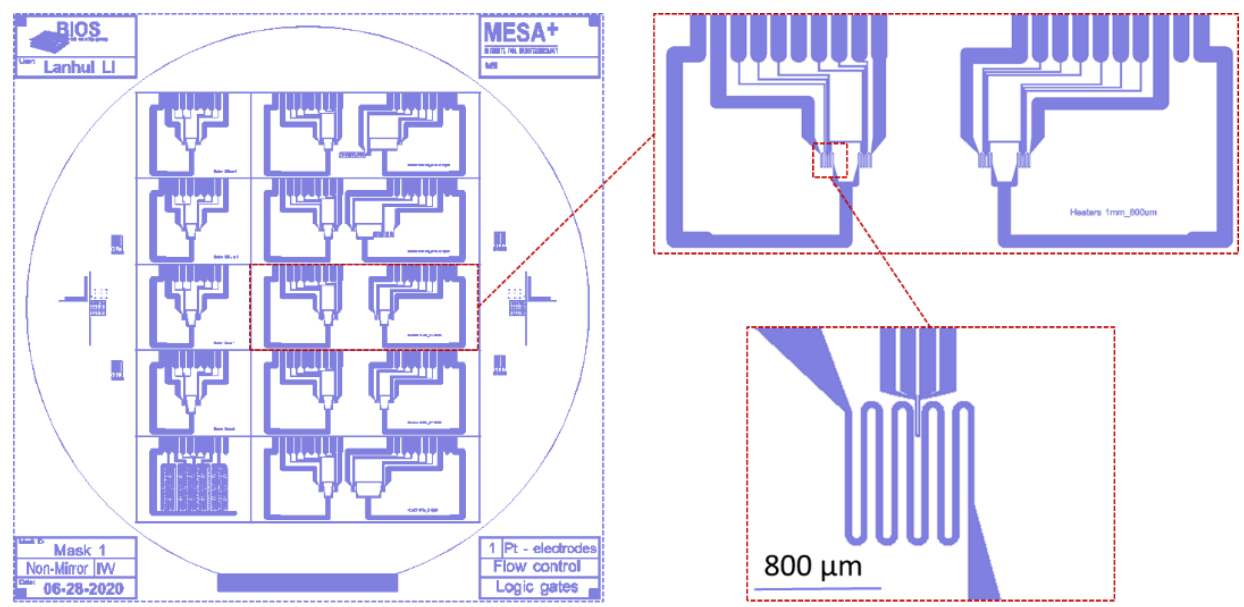

Figure D5 Design of heaters and sensors. Image on the top right shows the heater and sensorlayout used in chapter 5 . Image on the bottom right shows the detailed design of the heater and sensor.

D.5 Videos of pillared PNIPAm-g-PDMS stop valving, air-gate valving and liquid manipulation in chapter 5 .

Video 1 shows the capillary filling, thermal stimul us stop valving and liquid flow resumption in the pillared PNIPAm-g-PDMS microchannel. Video 2 shows the air-gate valving by temperature-controlled air expansion. Video 3 shows liquid loading, mixing and delivery in the channels. 


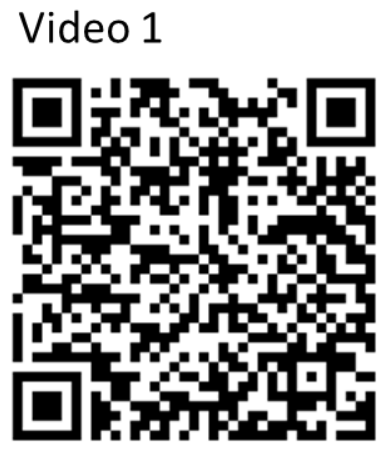

Video 2

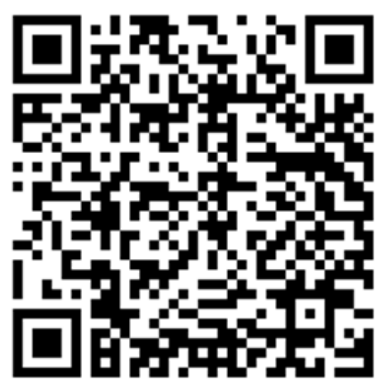

Video 3

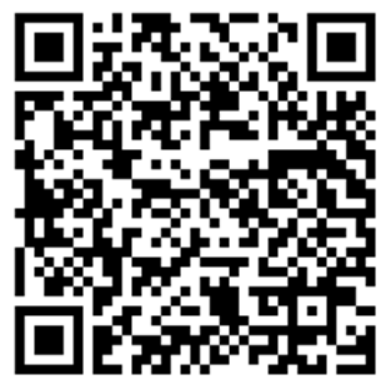




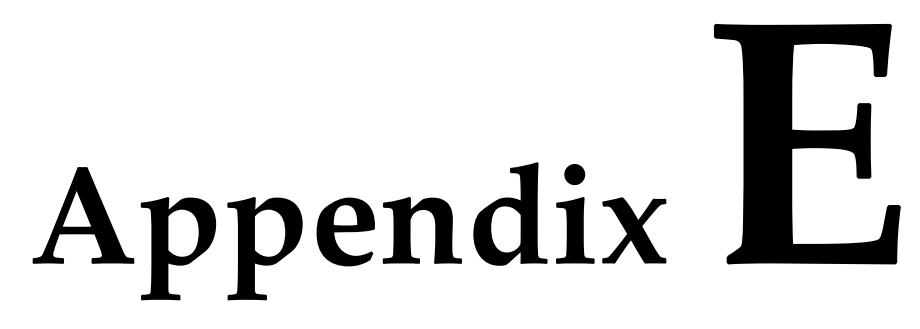

High Efficiency Hydrodynamic DNA Fragmentation in a Bubbling System 


\section{E.1 Gel image analysis}

Agarose gel images that were taken using a gel image system (BIO-BEST " $\mathrm{A}$ " series, SIM, America) were analyzed using MATLAB software to quantitate DNA fragment size and size distribution. The plot profile function for each electrophoresis lane is obtained as a curve of grayscale vs. migration distance. The gray scale of the gel image is corresponding to the fluorescence intensity. The size vs. migration distance calibration curve is obtained using the DNA markers (DL15,000 and DL 10,000 DNA markers, TaKaRa Biotechnology (Dalian) CO., Ltd, China). The fragment size of the major peaks is then determined based on the calibration curve of the migration distance. Then the curve of grayscale vs. fragment length (FL) can be obtained. The FL indicated on the $x$-axis (Figure E1 and Figure E2) is directly mapped using the markers. Figures E1 and E2 are the curves cor responding to Figures 6.2 and 6.4.

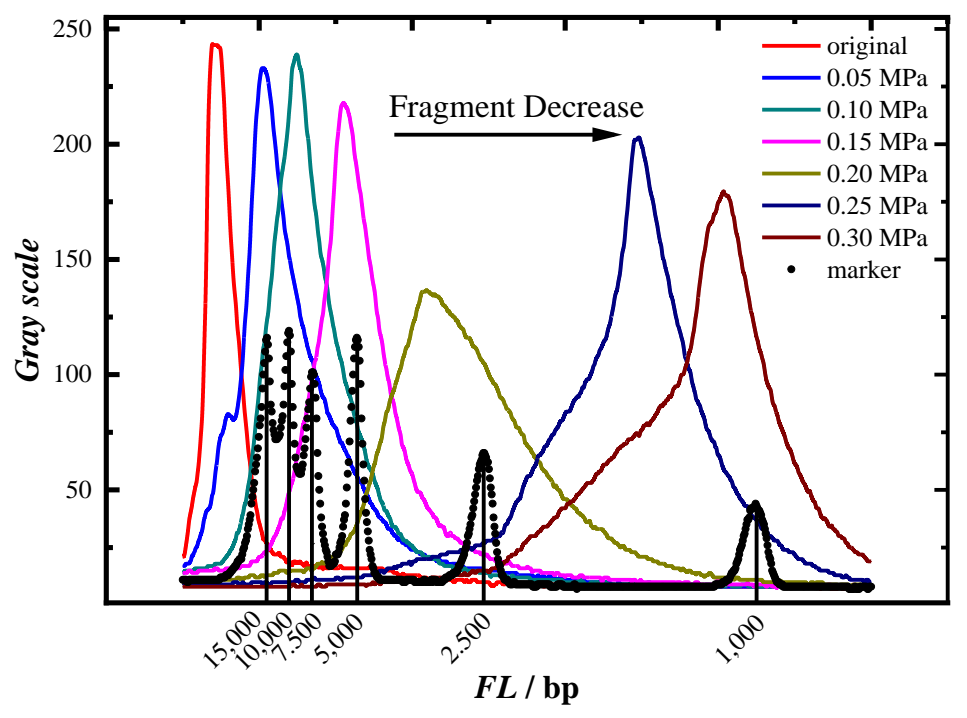

Figure E1 Fragment length distribution. DNA was fragmented at different pressure of 0 , $0.05,0.1,0.15,0.2,0.25,0.3 \mathrm{MPa}$ for $60 \mathrm{~min}$. DL 15000 that contains fragment leng th of 15 $\mathrm{Kbp}, 10 \mathrm{Kbp}, 7.5 \mathrm{Kbp}, 5 \mathrm{Kbp}, 2.5 \mathrm{Kbp}$, and $1 \mathrm{Kbp}$ was used as a marker. 


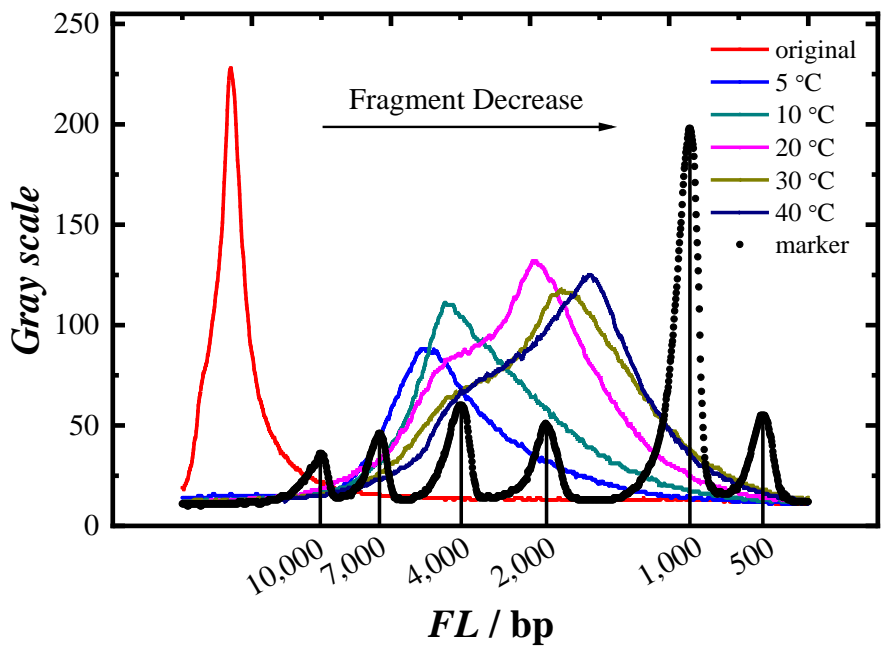

Figure E2 Fragment length distribution. DNA was fragmented at different temperature s of $5,10,20,30,40{ }^{\circ} \mathrm{C}$ for $60 \mathrm{~min}$. DL 10,000 that contains fragment length of 10,000 bp, $7,000 \mathrm{bp}, 4,000 \mathrm{bp}, 2,000 \mathrm{bp}, 1,000 \mathrm{bp}$, and $500 \mathrm{bp}$ was used as a marker.

\section{E.2 DNA fragmentation using a small glass vessel}

When the genomic DNAs were hydrodynamically sheared in a smaller glass vessel, as shown in Figure E3, shorter fragments with $F L<1000$ bp have been obtained.

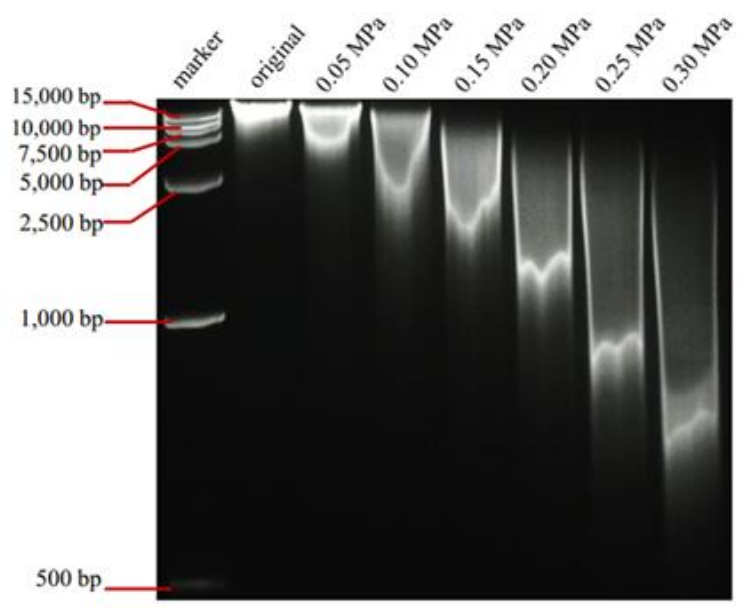

Figure E3 DNA fragment length $(F L)$ varies with applied gas pressure $(P)$. The shearing was carried out in a glass tube for $20 \mathrm{~min}$. 


\section{E.3 ssDNA digestion reaction.}

The digestion reaction solution contains a $1.2 \mu \mathrm{g}$ DNA sample (fragmented under different pressure); $6 \mu \mathrm{L}$ Exonuclease I ( $5 \mathrm{U} / \mu \mathrm{L}$, TaKaRa Biotechnology (Dalian) Co., Ltd, China); $2 \mu \mathrm{L}$ 10×Exonuclease I buffer and sterilized distilled w ater (to make the total volume up to $20 \mu \mathrm{L}$ ). The mixture w as incubated for 30 min at $37^{\circ} \mathrm{C}$. Then $10 \mu \mathrm{L}$ mixture was taken out to run gel electrophoresis experiments. The ssDNA was purchased from Sigma-Aldrich, Shanghai, China (Deoxyribonucleic acid, single-stranded from salmon testes, CAS: 68938-01-2). Figure E4 shows the gel images.

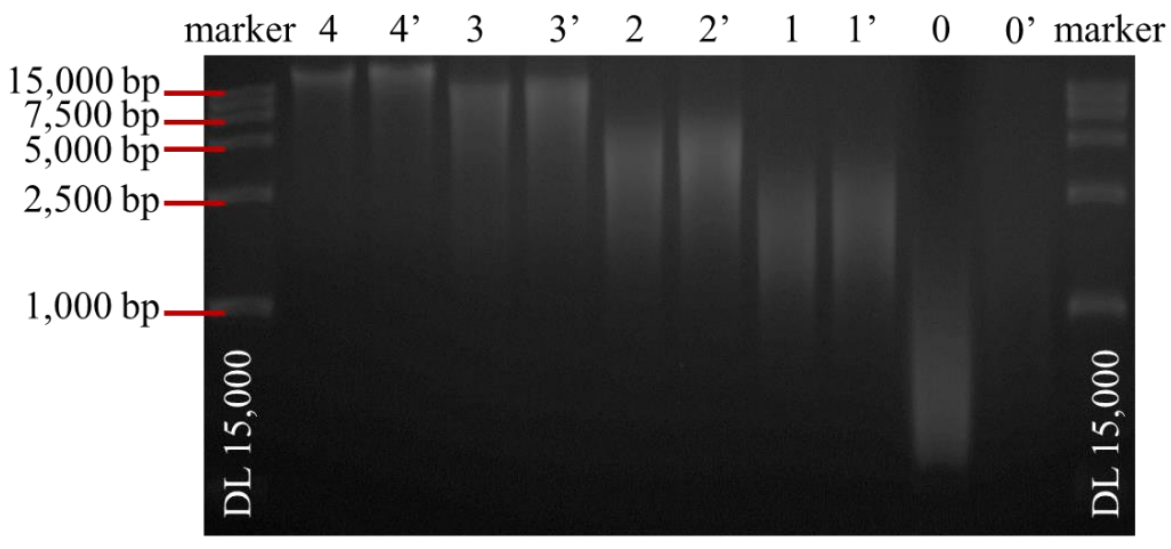

Figure $\mathrm{E} 4 \mathrm{Gel}$ images of ssDNA digestion reaction results: 0 and $0^{\prime}$ - ssDNA before and after digestion, 1 and $1^{\prime}$ - fragmented genomic DNAs (sheared at $0.10 \mathrm{MPa}, 60 \mathrm{~min}$ ) before and after digestion, 2 and $2^{\prime}$ - fragmented genomic DNAs (sheared at $0.20 \mathrm{MPa}, 60 \mathrm{~min}$ ) before and after digestion, 3 and $3^{\prime}$ - frag mented genomic DNAs (sheared at $0.30 \mathrm{MPa}, 60$ min) before and after digestion, 4 and $4^{\prime}$ - original DNA samples before and after digestion. The bubbling time is $60 \mathrm{~min}$ for all samples. Marker DL 15,000 was used to measure the size of the DNAs.

The concentration of dsDNA before and after digestion was measured by a spectrophotometer (Thermo Scientific NanoDrop 2000, USA). The results are shown in Table E1. Each data were averaged by 5 measurements. 
Table E1 Measurement results from Nanodrop 2000

\begin{tabular}{lllll}
\hline \multicolumn{3}{c}{ Before Digestion } & \multicolumn{2}{l}{ After Digestion } \\
\hline $\begin{array}{llll}\text { Pressure } \\
\mathrm{MPa}\end{array}$ & $\begin{array}{l}\text { dsDNA } \\
\text { concentration } \\
(\mathrm{ng} / \mu \mathrm{L})\end{array}$ & $\mathrm{OD}_{260} / \mathrm{OD}_{280}$ & $\begin{array}{l}\text { dsDNA } \\
\text { concentration } \\
\mathrm{ng} / \mu \mathrm{l}\end{array}$ & $\mathrm{OD}_{260} / \mathrm{OD}_{280}$ \\
\hline 0.0 & 82.56 & 1.86 & 82.64 & 1.37 \\
0.1 & 77.40 & 1.86 & 72.01 & 1.37 \\
0.2 & 80.10 & 1.85 & 72.37 & 1.5 \\
0.3 & 78.16 & 1.84 & 75.55 & 1.47 \\
\hline
\end{tabular}

\section{E.4 Fragmentation ofHerring Sperm DNA and Lambda DNA}

Herring Sperm DNAs (deoxy ribonucleic acid sodium salt from herring testes, CAS: 438545-06-3, Sigma-Aldrich, Shanghai, China) and Lambda DNAs (48.502 $\mathrm{Kbp}$, deoxyribonucleic acid, bacteriophage (Lambda Phage) from Escherichia coli (No. B600011, Sangon Biotech, Shanghai, China) were hydrodynamically sheared using this method (Figure E5). Herring Sperm DNA is a kind of natural DNA similar to Salmon Sperm DNA that we used in our experiments. The Lambda DNA is a ty pe of DNA with A-T rich sequences. ${ }^{1}$ If the hy drodynamic fragmentation happens easier at A-T region than $\mathrm{G}-\mathrm{C}$ region, specific DNA patterns should be observed. We carried out an experiment using this Lambda DNA, as show $n$ in Figure E5b. The fragmented DNAs show a smear pattern similar to the other two genomicDNAs (Salmon Sperm DNA(Figure 6.2 and 6.3) and Herring Sperm DNA (Figure E5a). Based on the current experimental results we obtained, thehydrodynamic DNA fragmented in the bubbling system seems not selective for A-Tregion at the parameters we have tested. 


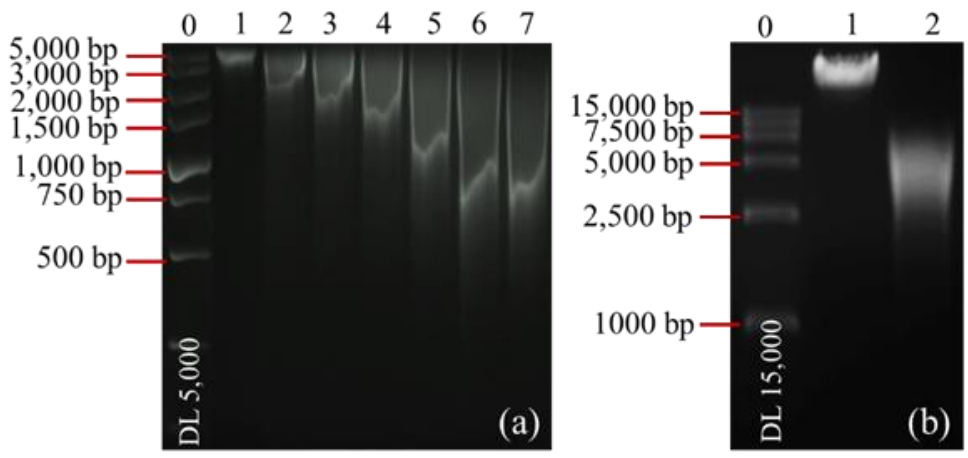

Figure E5 (a) Gel images of the DNAs fragmentation results of Herring Sperm DNA: 0 marker (DL 5,000), 1 - untreated Herring Sperm DNA (positive control), 2, 3, 4, 5, 6 and 7 - frag mented lambda DNAs for $30 \mathrm{~min}$ at $0.05,0.1,0.15,0.2,0.25,0.3 \mathrm{MPa}$, respectively. (b) Gel images of the DNA fragmentation results of lambda DNAs: 0 - marker (DL 15,000), 1 - untreated lambda DNA (positive control), 2 - fragmented lambda DNA for $30 \mathrm{~min}$ at $0.20 \mathrm{MPa}$.

\section{E.5 DNA fragmentation with silicon oil on the top}

Silicon oil was put on top of the DNA solution to investigate the effect of the bubble bursting on DNA fragmentation. $1.5 \mathrm{~mL}$ of $100 \mu \mathrm{g} / \mathrm{mL}$ DNA solutions with or without $1 \mathrm{~mL}$ silicon oil on the top of it were bubbled for $30 \mathrm{~min}$ at 0.10 MPa. The gel images of the original DNA sample (No. 1), bubbling with oil coverage (No.2) and without oil coverage (No.3) were demonstrated in Figure E6.

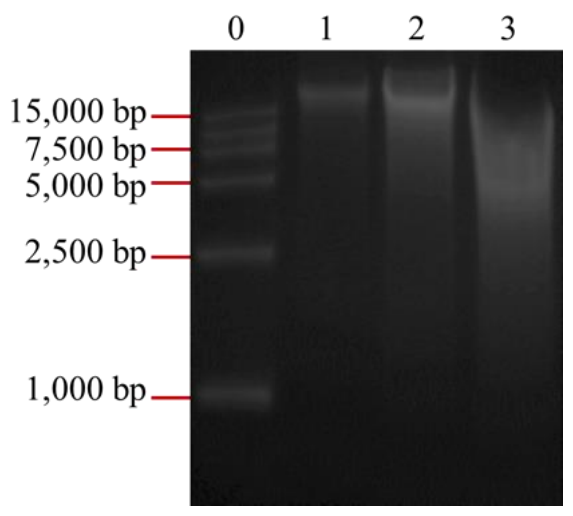

Figure E6 Gel images ofDNA fragmentation results with or without silicone oil coverage: 0 - marker (DL 15,000), 1 - original genomic DNA sample (positive control), 2 -frag mented 
DNA sample with oil coverage (30 min at $0.10 \mathrm{MPa})$, and 3 - fragmented DNA sample without oil coverage (30 $\mathrm{min}$ at $0.10 \mathrm{MPa})$.

\section{E.6 References}

1. Le Talaer, J. Y. \& Jeanteur, P. Preferential binding of E. coli RNApolymerase to A-T rich sequences of bacteriophage lambda DNA. FEBS Lett. (1971). 
Appendix E 


\section{Scientific Output}

\section{Peer-reviewed journal articles}

L. Li, E.Y. Westerbeek, J.C. Vollenbroek, S.J.A. de Beer, L. Shui, M. Odijk, and J.C.T. Eijkel. Autonomous capillary microfluidic devices with constant flow rate and temperature-controlled valving. In submission.

L. Li, L. Shui, M. Odijk, and J.C.T. Eijkel. On-chip detection of the alcohol concentration in beer. In preparation.

L. Li, L. Shui, M. Odijk, and J.C.T. Eijkel. Integration of air-pockets and pillared stop valve in a microchannel for capillary flow control. In preparation.

L. Li, Z. Yan, M. Jin, X. You, S. Xie, Z. Liu,A. van den Berg, J. C.T. Eijkel, L. Shui. In-Channel Responsive Surface Wettability for Reversible and Multiform Emulsion Droplet Preparation and Applications. ACS applied materials \& interfaces. 2019, 11(18), 16934-16943.

L. Li., M. Jin, C. Sun., X. Wang., S. Xie., G. Zhou, A. van den Berg, J.C.T. Eijkel \&

L. Shui. High efficiency hydrodynamicDNA fragmentation in a bubbling system. Scientificreports, 2017, 7(1), 1-9.

X. You, B. Wang, S. Xie, L. Li, H. Lu, M. Jin, X. Wang, G. Zhou \& L. Shui. Microfluidic-Assisted Fabrication of Monodisperse Core-Shell Microcapsules for Pressure-Sensitive Adhesive with Enhanced Performance. Nanomaterials, 2020, 10(2), 274 .

\section{Conference contribution}

L. Li, E.Y. Westerbeek, J.C. Vollenbroek, L. Shui, M. Odijk, and J.C.T. Eijkel. Autonomous capillary microfluidic devices with constant flow rate and temperature-controlled valving. 380-381. Paper presented at 24th International 
Scientificoutput

Conference on Miniaturized Systems for Chemistry and Life Sciences, $\mu$ TAS2020 (poster presentation, Virtual Event) 


\section{Samenvatting}

In dit proefschrift zijn op PDMS gebaseerde microfluïdische chips en systemen ontwikkeld. Deze zijn gefunctionaliseerd met PNIPAm, voor het genereren van druppels en het manipuleren van capillaire stromen. De werkingsprincipes van deze apparaten worden uitgelegd op basis van het tijdsafhankelijke 'wetting' (de affiniteit van een vloeistof voor een bepaalt oppervlak) van water of waterige oplossingen op PNIPAm-g-PDMS-oppervlakken. De schakelbare wetting werd gebruikt voor omkeerbare emulsievorming in op druppel vorming geb aseerde microfluïdische systemen en om afsluitkleppen te creëren in systemen die door capillaire werking worden aangedreven. Bovendien vertoonde het capillaire vulgedrag een constant vuldebiet, dat w erd bestudeerd en verklaard met behulp van een gemodificeerde Washburn-vergelijking waarbij rekening werd gehouden met de wrijving/frictie van de contactlijn. Het systeem werd met succes gebruikt voor het detecteren van alcohol in bier. Door vervolgens luchtkleppen te integreren in de op capillariteit gebaseerde microfluïdische apparaten, w erd een systeem met meer volledige functionaliteit verkregen voor het regelen van de capillaire stroming.

Hoofdstuk 2 beschrijft de methode van oppervlaktecoating die is gebaseerd op foto-enten van het thermo-responsieve polymeer PNIPAm op PDMS. Deze coatingmethode dient ook als basiscoatingtechnologie in de hoofdstukken 3, 4 en 5. Er w erd een omkeerbare thermo-responsieve w etting bereikt. Bij het variëren van de PNIPAm-g-PDMS-oppervlaktetemperatuur tussen 25 en $38^{\circ} \mathrm{C}$, kan de wetting van het oppervlak om keerbaar w orden geregeld tussen hydrofiel (water aantrekkend, $\mathrm{CA}=26^{\circ}$ ) en hydrofoob (water afstotend, $\mathrm{CA}=106^{\circ}$ ). De dynamische afstemming van de wetting van het kanaaloppervlak stelde ons in staat om omkeerbare olie-in-water $(\mathrm{O} / \mathrm{W})$ en water-in-olie $(\mathrm{W} / \mathrm{O})$ emulsies in dezelfde chip te verkrijgen. Dubbele O/W/O- en W/O/W-emulsies kunnen worden gevormd door ofw el het kanaaloppervlak lokaal te bedekken met PNIPAm of door het met PNIPAm geënte kanaal lokaal te verwarmen. De apparaten werden gebruikt om kern-schil (core-shell) microcapsules te produceren met verschillende schil-diktes. Dit systeem kan dienen als een nuttig platform om dynamische processen van omkeerbare meerfasige micro / 
nanofluïdische verschijnselen op oppervlakken of in besloten microruimtes te bestuderen.

In hoofdstuk 3 werd dezelfde ent-technologie gebruikt in een op PDMS gebaseerd capillair-microfluïdisch systeem. Sterk tijdsafhankelijke w etting van w ater op het PNIPAm-g-PDMS-oppervlak werd w aargenomen en onderzocht door middel van contacthoekmetingen. De omkeerbare wetting werd gebruikt om een afsluitklep te creëren om de capillaire stroom te regelen, aangezien water het kanaal automatisch vult door capillaire krachten en kan worden gestopt door lokaal de oppervlakte wetting van de kanaalwanden te regelen door de temperatuur te veranderen van $20^{\circ} \mathrm{C}$ naar $36^{\circ} \mathrm{C}$. Bovendien werden in dit systeem constante capillaire vulstroomsnelheden (capillair vulgedrag dat zich niet gedraagt volgens de klassieke Washburn vergelijking) variërend van $1 \mu \mathrm{m} / \mathrm{s}$ tot $240 \mu \mathrm{m} / \mathrm{s}$ waargenomen. We verklaarden dit fenomeen door middel van w rijving op de contactlijn en beschreven het met behulp van een gemodificeerde Lucas-Washburn-vergelijking. In deze vergelijking w ordt rekening gehouden met de w rijving van de contactlijn, de oppervlaktespanning en de viscositeit om het capillaire vulgedrag te bepalen. Wanneer de w rijving van de contactlijn dominant is, zullen we constante vulstroomsnelheden in het kanaal verkrijgen. Als er geen contactlijnwrijving is, volgt het vulgedragde W ashburn-vergelijking met de vullengte evenredig met $\sqrt{ } \mathrm{t}$. Het constante capillaire vuldebiet in PNIPAm-g-PDMS-apparaten biedt een stabiel en controleerbaar vloeistofstroomveld dat gunstig zou kunnen zijn voor het incubatieproces in immunoassays. Het kan ook w orden geb ruikt als een vloeistofdoseerplatform. In een kanaal met een bepaalde temperatuur en geometrie is het vloeistofvolume alleen afhankelijk van de vultijd. De interactie tussen water en PNIPAm kan w orden beïnvloed door een kleine hoeveelheid alcohol.

In hoofdstuk 4 onderzochten we de wetting van water/ethanolmengsels op PNIPAm-g-PDMS-oppervlak en bestudeerden we het effect van het ethanolgehalte op het vulgedrag van water/ethanolmengsels in PNIPAm-gPDMS-microkanalen. Water/ethanolmengsels van $0 \%$ tot $4 \%$ molaire fractie vertonen een constant vuldebiet in PNIPAm-g-PDMS-microkanalen met een debiet dat afhankelijk is van de ethanolconcentratie. Water/ethanolmengsels met een hogere molaire fractie vertonen een vulgedragsovergang van constant debiet naar Washburn-gedrag. In algemene termen w ordt het vloeistofvulgedrag in PNIPAm-g-PDMS-kanalen bepaald door de vloeistof-PNIPAm-interactie, vloeistofoppervlaktespanning en viscositeit. De afhankelijkheid van de 
vullingsgraad van het ethanolgehalte maakt het systeem tot een veelbelovend hulpmiddel voor het detecteren van lage alcoholconcentraties in alcoholische dranken door simpelweg de vultijden te vergelijken. Op deze manier werden Grolsch-bieren met $0 \%$, $2 \%$ en $5 \%$ ABV binnen 10 seconden van elkaar onderscheiden.

In hoofdstuk 5 hebben we de prestaties van de PNIPAm-g-PDMS-afsluitklep verder verbeterd. Door vrijstaande pilaren op het oppervlak toe te voegen, hebben we een oppervlak verkregen met een groter wetting bereik. Nu werd ook een afsluitklepfunctie verkregen in nieuw gecoate chips. De afsluitklepfunctie in een capillair microfluïdisch systeem is niet voldoende voor programmeerbare vloeistofmanipulatie, omdat de vloeistofstroom niet opnieuw kan worden gestopt. Daarom hebben we lucht aangedreven afsluitkleppen geïntegreerd met de PNIPAm-g-PDMS afsluitkleppen in één w erkend systeem. De luchtklep kan w orden bediend door thermische uitzetting van de lucht in luchtzakken aan de zijkanten van de hy drofobe afsluiter. Zow el de hy drofobe klep als de luchtklep kunnen w orden bediend in het temperatuurbereik van $20-38^{\circ} \mathrm{C}$. Een systeem met 4 individuele units werd vervolgens ontwikkeld en getest met het vermogen voor vloeistofmanipulatie, zoals het inbrengen van vloeistoffen, het mengen van vloeistoffen en het afgeven van vloeistoffen door de individuele kleppen achtereenvolgens in w erking te stellen.

Hoofdstuk 6 presenteert een snelle, gemakkelijke en robuuste DNAfragmentatietechnologie met behulp van een bubbelsysteem. Door N2-gas via een buisje in een waterige DNA-oplossing te brengen, zalzich een bel vormen bij de opening van de buis, deze bel stijgt op, zet uit en voegt samen aan het vloeistofoppervlak totdat deze tenslotte barst. Dit resulteert in de vorming van een vloeistofstraal. Tijdens verloop vanaf de vorming van bellen tot aan het barst proces wordt genomisch DNA geabsorbeerd aan het gas-vloeistofgrensvlak (dunne bellenfilm), w aarbij het DNA zich uitstrekt en uit elkaar geschoven door de hydrodynamische afschuifkracht die w ordt gegenereerd tijdens het barsten van de bellen en de jetvorming. Het genomische DNA werd uit elkaar geschoven tot het bereik van 1,0-10 Kbp met hoge reproduceerbaarheid. De methode is eenvoudig, goedkoopen gebruiksklaar voor veel laboratoria als protocol voor de voorbereiding van monsters voor DNA-analyse. 
Samenvatting 


\section{Funding and Contributions}

\section{Funding}

The research works presented in this thesis were supported by Guangdong Province Basic and Applied Research Fund (Grant No. 2019B1515120037), the Natural Science Foundation of Shaanxi Province (2020JQ-404), and the China Scholarship Council(CSC) (Grant no. 201806750019).

\section{Contributions}

This thesis is based on the collaboration between the BIOS Lab-on-a-Chip group of the University of Twente (BIOS/UT) headed by Prof. dr. Albert van den Berg and the Electronic Paper Display Institute of South China Normal University (EPDI/SCNU) headed by Prof. dr. Guofu Zhou. The research works described in this thesis were achieved through close collaboration of people from different research fields. Specifically Prof. dr. Jan C.T. Eijkel (BIOS/UT) and Prof. dr. Lingling Shui (EPDI/SCNU) contributed to all the chapters both directly via reviewing the thesis and the published articles and indirectly via fruitful weekly discussion and supervision. Prof. dr. ir Mathieu Odijk (BIOS/UT) provided input to chapters 3,4 , and 5 via reviewing the thesis and the research articles together with daily supervision and fruitful weekly discussions. Dr. Sissi de beer (Materials Science and Technology of Polymers, University of Tw ente) provided input in chapters 3 and 4 in the form of thorough discussions and reviewing the thesis and research articles.

\section{Chapter 2}

Prof. Mingliang Jin provided fruitful discussions and critical evaluation of the experimental work. Dr. Zhibin Yan contributed to experimental setup buildup, partial data collection, and analysis. I would like to thank Xiangshen You, Shuting Xie for their helpin conducting the lab work.

\section{Chapter 3}

This chapter is the results of collaboration with Jeroen C. Vollenbroek and Eiko Y.W esterbeek in BIOS Lab-on-a-Chip group. Jeroen helped with building up the 
experimental setup and Eiko conducted the simulation work. Both of them contributed to this work via fruitful discussions and writing/reviewing the research article. I would like to thank Dr. Vasilis Papadimitriou for his contribution to data processing. Thanks to Johan G. Bomer for fabrication support in thecleanroom. I alsow ant to thank Dr. H.T.M. van den Ende (Physics of Complex Fluids, University of Twente) for the discussion about the capillarity phenomenon and Vasilis Papadimitriou and Joshua Loessberg-Zahl for thorough discussions about capillary valves.

\section{Chapter 4}

I would like to thank dr. Sissi de Beer for the thorough discussion on the cononsolvency of PNIPAm in w ater/ethanol mixture.

\section{Chapter 5}

I w ould like to thank Henk B. Waayer from Techno Centre for Education and Research at the University of Twente (TCO-UT) for designing and fabricating the PCB board. Thanks to Rindert R. Nauta, and Akken Sahin (TCO-UT) for making the LabView program for temperature control. I also w ant to thank Jeroen C. Vollenbroek for help with PCB problems. Also many thanks to Johan G. Bomer for the cleanroom support!

\section{Chapter 6}

This chapter resulted from the collaboration with Chenglong Sun from the South China Sea Institute of Oceanology of Chinese Academy of Sciences. Chenglong Sun helped with the DNA analysis. Thanks to Prof. Xiaoxue Wang for the fruitful discussion and the review of the paper. I also w ant to thank Prof. Mingliang jin for the research ideas and the thorough discussion in this work. Thanks to Yany un Peng, Tao He, Angran Zhang, and Shuting Xie for the technical support. 


\section{Acknowledgements}

Welcome to the last but most important part of this thesis! After a long time $w$ riting and revising each chapter, I still feel I'm in a dream! Over the past 3 years and 7 months, I have had two academic homes, one in the Electronic Paper Display Institute of South China Normal University and one in the BIOS Lab-ona-chipgroup at the University of Tw ente! I always consider myself very lucky to meet and work with people of these two communities. Without the help and support from those lovely people, the w orks presented in this thesis can not be finished and I can't have such a colorful life in my PhD. Now it's time to look back to the past memorable years and express my gratitude and my appreciation to all those in the next lines.

I w ould like to start with my supervisor, prof. dr. Lingling Shui. Lingling, it has been almost 7 years since the day you started supervising me during my master's study. I still remember the questions you asked me during my postgraduate interview. After that, I started a long journey in the w orld of microfluidics and I think it will last the rest of my life. Thank you for all the knowledge you transferred to me and also for all kinds of support in my daily life. Thank y ou for encouraging me to explore my life abroad and to pursue my PhD. And thank you for alw ays ready to be a good listener when I w ant to talk. I really enjoy my time w orking with you.

Then my deep gratitude must go to my supervisor, prof. dr. Jan Eijkel. The first time I met with you, Jan, you were my supervisor's supervisor. I remember when I w as presenting my results to you, you always ask 'why?', a lot of 'w hy?'. I was impressed by your extraordinary and multidisciplinary knowledge. And I feel so lucky to be one of your PhD students. You can alw ays explain a complex phenomenon in a very simple and understandable way for outsiders. Every time after our meeting, I w ill take some A4 papers from you, where you draw pictures to explain a phenomenon or your thoughts. Those pictures were finally become text or figures here and there in my thesis, I appreciate them a lot. Jan, thank you for always ready to work together with me when I'm stuck and for always encouraging me when I'm not motivated. Thank you for your patience and 
kindness to people and your happiness and curiosity about life. Ilearn a lot from you, both in scientific work and in life. And recently I found I started to ask people 'w hy?' and myself as well, when reading or listening to others w orks. You influent me a lot!

Next, I w ould like to thank my co-supervisor, prof. dr.ir. Mathieu Odijk. My journey in BIOS started with capillary valves, in which a precisely controlled local heating technology is needed. I never doubt I will have any problem with this as you showed me all the microheater designs your students made after our first meeting. Mathieu, thank you for bringing me into the w orld of electro engineering. And thank you for all your thoughts and comments during our meeting from an engineer's point of view. Thank you for all the encourages and effor ts you made to make my life easier. Your excellent idea about the beer sensor, in the beginning, we planned to make it a Friday afternoon experiment for fun. And see how much we explored now, I learned a lot from this w ork! And there is one thing I still need to study I think, the logic gates!

My special thanks will address to prof. dr. Albert van den Berg. Thank you for leading BIOS a multidisciplinary working environment with much fun and happiness. The w orkweeks, barbecues, mountain-biking.... I enjoy my time in BIOS a lot. BIOS is a w ell-organized and structured research group with almost every thing a researcher needs to conduct their w ork (technical support, basic know ledge, nice facilitates...). I feel lucky to be a member of such a great team. Also, thanks to your efforts together with Jan, Guofu and Lingling, for founding thejoint laboratory 'LOTS' between SCNU and UT. That's where my story with BIOS begins.

Then my sincere thanks must go to prof. dr. GuofuZhou, the leader of Electronic Paper Display Institute. I remember years ago when you know I'm going to do a $\mathrm{PhD}$, you encouraged me to work hard and to be an independent scientist. Every time we see each other, you care about our research w ork and our life. Thank you for all your efforts to make EPDI stronger and more international, thus young researchers will have chances to explore the world.

I would like to thank my promotion committee members, prof. dr. Akihide Hibara, prof. dr Yanbo Xie, dr. Emmanuel Delamarche, prof. dr. Frederic $\mathbf{R}$. Wurm, prof. dr. Han Gardeniers and dr. Sissi de Beer, for evaluating and approving my thesis for public defense. I'm looking forward to your questions. 
I w ould like to thank dr. Wouter Olthuis, prof. Loes I. Segerink, dr Tim J. Segers and dr. Sergii Pud. Wouter, you have given a lecture on electrochemical sensors in the lab on chip course, where I learned the sensing methods, I hope in the future, I will have a chance to use one of them in my research work. Loes, Tim and Sergii, thank you for the feedback after the Monday morning talks.

I'd like to extend my thanks to Hermine Knol-de Vries, Johan Bomber, Paul ter Braak, Hans de Boer, Lisette V.Knippenborg, Pieter H. Huchshorn, Eddy L. de Weerd. Hermine, thank you for all kinds of help in administration. Withoutyour help, things wouldn't go so smoothly! Johan, thank you for your great help in the cleanroom. Not only in the fabrication process but also for characterization and surface treatment. Thank you for making the Peltierelement, which has become a very important part of my setup. Paul, thank you for taking care of the lab safety and ordering chemicals. So everyone can work in a safe, clean environment. Also thank you for always be cheerful both in the coffee cornerand the lab. I w ant to thank Hans for helping with technical problems. And thank Lisette for ordering chemicals. We had some problems or dering Benzolphenone from Sigma-Aldrich but in the end, we got it from Thermo Fisher! Many thanks to Pieter for alw ays be patient in helping me finding chemicals and introducing the new label printing system. Eddy, you alw ays come to my office and say goodbye at the end of the day, which makes me feel very warm, thank you!

I w ant to thank my officemates, Alessia, Jeroen and Rob. Alessia, you are the first Italian I have met in my life. Apparently, you gave me a very good impression of Italians. The first day I met you in the office, you make me feel you are my old friend! In the past 2 years, we shared a lot of happy moments. Thank you for waking me up and cheering me up when I was writing my thesis, and thank you for always reminding me of things. Jeroen, you are an important person in our group (both Alessia and I agree with that) as we see people always come to our office, looking for you for help. I then became one of them. Thank you for being always ready to help with building my experimental setup, explaining the electronic circuit, solving PCB problems, and Dutch translation stuff. Rob, you alw ays get candies when you ordering things and then you will share them with us. And you are another electrical engineer in our office who explain things to me patiently when I w as bothering you with all kinds of strange questions. Currently, you are having off-times at home. I hope you enjoy your life and come back to BIOS soon. Then we will have more candies! (:) 
I w ould like to thank Alessia and Luca for accepting to be my paranymphs. I'm not sure if the defense will be fully digital or not, but I know you two will bring megood luck!

Eiko, thank you for your contribution to our paper. I was impressed by your simulation skills and your amazing cooking skills a lot. I also would like tothank Vasilis and Joshua for our nice discussion about capillary valves. And special thanks to Vasilis for making the Matlab code for velocity analysis in my system. Many thanks to Douwe for help with PCB problems. And thanks to Elsbeth for making COC chips and providing pillar mold to me for testing.

To Jasper and Alessia, thank you for organizing the Monday morning talks (including few Thursday afternoon talks).

Special thanks to dr. Burcu Gumuscu and dr. Floris van den Brink for supervising me when I w as doing my master's internship in BIOS.

I w ould like to thank those international chefs: Hai, Hoa, Christina, Vasilis, Eiko, Douwe, Juan, Masha, Alessandro, Alessia, Luca for organizing cooking activities and enjoying nice food together before covid. I also want to thank Edo, Ketki, Koen who came up with online Friday afternoon cooking activities to make our life more colorful. Special thanks to Edo for organizing the Friday afternoon talks and cooking activities.

Many thanks to those runners: Anke, Tanja, Stella, Christina, Rob, Hoa, Masha, Ketki, Koen, Pascal, Alessandro, Luca for doing exercise and making our life healthier together. Thanks to Tanja, Nienke and Heleen for the wine tasting and soda tasting. I havenever drink that much w ine in my life.

Thanks to the rest of the current BIOS members (names in no particular order): Esther, Dasha, Ruben, José, Job, Jorien, Dodo, Rajendra, Martin for allkinds of help and for making a lot of fun during coffee/lunch breaks, running the Batavierenrace and other BIOS activities. I feel very happy being met $w$ ith all of you! Probably I will forget to mention some names, please forgave me and I'm sure you are in my thanks!

I w ould like to thank prof. dr. Sissi de Beer and Guido Ritseman van Eck from Materials Science and Technology of Polymers Group for fruitful discussion on the wetting behavior of PNIPAm brushes. I'm looking forward to the MD simulation results. I also w ant to thank prof. dr. Frieder Mugele and dr. Dirk 
van den Ende from the Physics of Complex group for giving the Capillarity Phenomenon courses and leading me to the w ord of capillary action.

I also w ant to thank my Chinese friends who studied or are studying in the Netherlands (names in no particular order): Wu Hao, Xie Lingyun, Su Shaoqiang, Chen Lin, Yang Yajie, Zhan Yuanyuan, Zhang Weixin, Duan Feibo, Yao Junxiang, An Qi, Lin Min, Li Minsi. Thank you for all the good times we spent together and for the nice talks about science and life.

I w ould like to thank prof. dr. Mingliang Jin and dr. Zhibin Yan in SCNU for our discussions on my project. And many thanks to Shuting Xie, Han Lu, Ting Zhang, Jieping Cao for help with preparing documents that needed for my graduation at SCNU.

Last but not least, my great thanks to my Family! Mom and Dad, thank you for your love and care. And thank you for always believing in me and supporting my choices. Thanks to my sister and brother-in-law. Because of you, I have no w orries to pursue my study abroad. To my nephew, Yuanrui Chen, I w ish you grow up healthy and happy!

\section{Lanhui}

April 2021, Enschede 



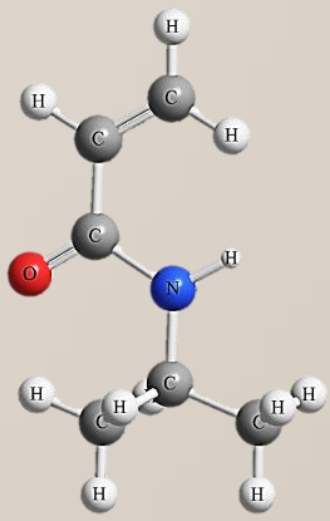

ISBN: 978-90-365-5177-9 